\title{
The Plectic Weight Filtration on Cohomology of Shimura Varieties and Partial Frobenius
}

\author{
Zhiyou Wu
}

Max Planck Institute for Mathematics, Bonn 53111, Germany; E-mail: wuzhiyou1992@gmail.com.

Received: 5 August 2020; Revised: 6 February 2021; Accepted: 11 March 2021

2020 Mathematics Subject Classification: Primary - 16W10; Secondary - 16D50

\begin{abstract}
We prove that there is a natural plectic weight filtration on the cohomology of Hilbert modular varieties in the spirit of Nekovár and Scholl. This is achieved with the help of Morel's work on weight t-structures and a detailed study of partial Frobenius. We prove in particular that the partial Frobenius extends to toroidal and minimal compactifications.
\end{abstract}

\section{Contents}

1 Introduction $\quad 1$

2 Morel's weight t-structure $\quad 5$

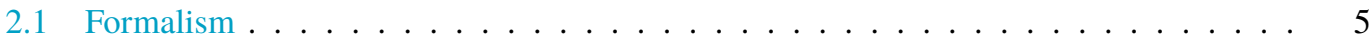

2.2 Applications to Shimura varieties . . . . . . . . . . . . . . . . 10

3 PEL moduli problems $\quad 15$

3.1 Kottwitz's PEL moduli problems . . . . . . . . . . . . . . . . . . . . 15

3.2 Similitude PEL moduli problems . . . . . . . . . . . . . . . . . . . 17

3.3 The partial Frobenius . . . . . . . . . . . . . . . . . 23

4 Hilbert modular varieties $\quad 27$

4.1 Basics . . . . . . . . . . . . . . . . . . . . 27

4.2 The weight spectral sequence . . . . . . . . . . . . . . . . . . 29

4.3 The plectic weight filtration . . . . . . . . . . . . . . . 32

5 Toroidal compactifications and the partial Frobenius $\quad 40$

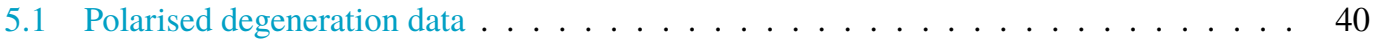

5.2 PEL degeneration data . . . . . . . . . . . . . . . . . . . . . 48

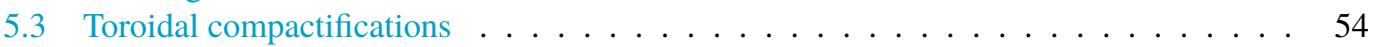

5.4 Partial Frobenius extends to toroidal compactifications . . . . . . . . . . . . 61

5.5 Partial Frobenius extends to minimal compactifications . . . . . . . . . . 81

\section{Introduction}

Nekovár and Scholl recently proposed in [26] a program on plectic theory, which is about some hidden symmetries of Shimura varieties. The theme of this article is to exploit some of these hidden symmetries and provide evidence for their conjectures. More precisely, Nekovár and Scholl observed that when the

(C) The Author(s), 2021. Published by Cambridge University Press. This is an Open Access article, distributed under the terms of the Creative Commons Attribution licence (http://creativecommons.org/licenses/by/4.0/), which permits unrestricted re-use, distribution, and reproduction in any medium, provided the original work is properly cited. 
group of a Shimura variety $X$ is of the form $\operatorname{Res}_{F / \mathbb{Q}} G$ with $F$ totally real, the cohomology of $X$ has extra structures. This is most easily observed in the (Betti) intersection cohomology of (minimal compactification of) Shimura varieties, in which case we have

$$
\begin{aligned}
& I H^{*}\left(X^{\min }(\mathbb{C}), \mathbb{C}\right)=H_{(2)}^{*}(X(\mathbb{C}), \mathbb{C})=\underset{\pi}{\oplus} H^{*}\left(\mathfrak{g}, K_{\infty} ; \pi_{\infty}\right) \otimes \pi_{f}^{K_{f}}= \\
& \underset{\pi}{\oplus} \underset{v \mid \infty}{\otimes} H^{*}\left(\mathfrak{g}_{v}, K_{\infty, v} ; \pi_{v}\right) \otimes \pi_{f}^{K_{f}}
\end{aligned}
$$

where the first equality is the (proven) Zucker's conjecture, $\pi$ ranges over irreducible $L^{2}$ automorphic representations of the group $\operatorname{Res}_{F / \mathbb{Q}} G$ and the last equality follows by applying the Kunneth theorem for $(\mathfrak{g}, K)$-cohomology to $\pi_{\infty}=\underset{v \mid \infty}{\otimes} \pi_{v}$. Because each $(\mathfrak{g}, K)$-cohomology $H^{*}\left(\mathfrak{g}_{v}, K_{\infty, v} ; \pi_{v}\right)$ is equipped with a Hodge structure of type $\left(p_{v}, q_{v}\right)$, we see that $I H^{*}\left(X^{\min }(\mathbb{C}), \mathbb{C}\right)$ is a sum of refined Hodge structures of type $\underset{v \mid \infty}{\otimes}\left(p_{v}, q_{v}\right)$; that is, plectic Hodge structures. A remarkably similar structure appears in the etale cohomology, at least in the case of Hilbert modular varieties, which suggests that it is motivic in nature. This motivates the question of explaining this extra structure.

Nekovár and Scholl proposed that the Shimura variety extends to a variety defined over $\operatorname{Spec}\left(k_{\text {plec }}\right)$, where $\operatorname{Spec}\left(k_{\text {plec }}\right)$ is a (product of) symmetric product of $\operatorname{Spec}(k)$ over $\mathbb{F}_{1}$, the field with one element. Obviously, this does not make sense because we do not have a good theory of $\mathbb{F}_{1}$. However, this heuristic allows us to guess what extra structures we can expect on the cohomology, which sometimes can be established directly. In particular, we expect that for noncompact Shimura varieties of type $\operatorname{Res}_{F / \mathbb{Q}} G$, the Betti cohomology has a natural plectic weight filtration, which is a $\mathbb{Z}^{d}$-indexed filtration whose graded pieces have pure plectic Hodge strutures as we observed using $(\mathfrak{g}, K)$-cohomology. What we prove in this article is that this is true in the special case of Hilbert modular varieties. Before explaining more about the results, we remark that the plectic conjectures have powerful arithmetic consequences on special values of L-functions; see [26] for details.

Let us first recall how we detect the classical weight filtration on a smooth nonproper complex variety $X$. Using Nagata embedding and resolution of singularities, we can find an open embedding $j: X \hookrightarrow \bar{X}$ into a proper smooth variety with $\bar{X} \backslash X$ union of normal crossing divisors. Then, as observed by Deligne [8], the weight filtration is detected using the filtration on $R j_{*} \mathbb{C}$ induced by the standard truncation $\tau_{\leq a} R j_{*} \mathbb{C}$, and the graded pieces of the weight filtration are detected using cohomology of strata of $\bar{X}$. More precisely, we have a spectral sequence induced by the filtration $\tau_{\leq a} R j_{*} \mathbb{C}$,

$$
E_{1}^{p, q}=\mathbb{H}^{p+q}\left(\bar{X}(\mathbb{C}), \tau_{\geq-p} \tau_{\leq-p} R j_{*} \mathbb{C}\right) \Rightarrow H^{p+q}(X(\mathbb{C}), \mathbb{C}),
$$

which is nothing but the (reindexed) Leray spectral sequence for $j$. The graded sheaves $\tau_{\geq-p} \tau_{\leq-p} R j_{*} \mathbb{C}$ are supported on the strata defined by intersections of boundary divisors, and the weight filtration is a shift of the converging filtration of the spectral sequence.

When $X$ is a Shimura variety of type $\operatorname{Res}_{F / \mathbb{Q}} G$, we can find an explicit $\bar{X}$ using toroidal compactifications. However, we cannot use them to detect the plectic weight filtration because toroidal compactifications are not 'plectic'; in particular, their strata possess no plectic structures on their cohomology. Our strategy is to look at the minimal compactification $X^{\text {min }}$ of $X$ instead, and what we gain is that the strata are now again Shimura varieties of type $\operatorname{Res}_{F / \mathbb{Q}} G$; hence plectic. This is a highly singular proper variety, and $\tau_{\leq a} R j_{*} \mathbb{C}$ is not a reasonable object to consider. The way to approach it is to use Morel's weight t-structures ([20]) in place of the standard t-structures. The formalism gives us a new truncation $w_{\leq a} R j_{*} \mathbb{C}$, giving rise to a spectral sequence of Hodge structures

$$
E_{1}^{p, q}=\mathbb{H}^{p+q}\left(X^{\min }(\mathbb{C}), w_{\geq-p} w_{\leq-p} R j_{*} \mathbb{C}\right) \Rightarrow H^{p+q}(X(\mathbb{C}), \mathbb{C})
$$

first observed by Nair in [24]. Note that Morel's formalism only makes sense in a theory with good notion of weights and perverse sheaves, and we have to use the derived category of mixed Hodge 
modules here. It is not hard to see that $w_{\geq-p} w_{\leq-p} R j_{*} \mathbb{C}$ decomposes into shifted simple Hodge modules strictly supported on (closure of) strata of $X^{\text {min }}$ and can be made explicit with the help of Burgos and Wildeshaus's results ([5]). Moreover, these simple summands are automorphic in the sense they are associated to algebraic representations of the groups associated to the strata they support. Now $E_{1}^{p, q}$ is a sum of intersection cohomology of plectic Shimura varieties with automorphic coefficients; the same computation as before using $(\mathfrak{g}, K)$-cohomology on twisted automorphic representations shows that it possesses plectic structures.

To proceed further, we have to know whether the spectral sequence detects the weight filtration and how we can extract the plectic weight filtration from it. Unfortunately, the answer to the first question is no in general, though it is true in the Hilbert modular case. The problem is that the graded pieces of the filtration are not necessarily pure but direct sums of pure Hodge structures possibly of different weights. It is a coincidence that in the Hilbert modular case this does not happen. On the other hand, to find the plectic weight filtration it is not necessary to know the weight filtration a priori, and the spectral sequence does help with our purpose.

To motivate the strategy, let us recall that there is another way to detect weights, namely, using Frobenius weights. By spreading out the variety, we can assume that it is defined over a finitely generated $\mathbb{Z}$-algebra and reduce it to a variety defined over a finite field. Then the Weil conjecture proved by Deligne [9] tells us that the $l$-adic cohomology has a weight filtration defined by Archimedean places of Frobenius eigenvalues. Using comparison theorems and base change or nearby cycles, we can find the weight filtration on Betti cohomology using finite fields. It is necessary to check that the new weight filtration is the same as the previously defined one, and this is proved by observing that the Frobenius acts on the spectral sequence (1) through the comparison isomorphism and has the right Frobenius weight on each $E_{1}^{p, q}$.

In the plectic case, we expect that there are plectic Frobenius weights in some reasonable sense, and the above classical method can be applied to find the plectic weight filtration. Fortunately, morphisms called partial Frobenius have been defined and studied in the literature ([25]). These are decompositions of the usual Frobenius, and their eigenvalues are naturally expected to give plectic Frobenius weights; hence the plectic weight filtration. To fulfil the expectation, we have to prove that the partial Frobenius extends to the minimal compactification and induces a morphism on the spectral sequence (2). This is achieved through toroidal compactifications. Indeed, we prove firstly that the partial Frobenius extends to toroidal compactifications, using Lan's universal property of toroidal compactifications ([18]). To check the universal property, we have to make full use of the degeneration data of semi-abelian varieties constructed by Faltings-Chai and Lan [11]. Then we prove that the extended partial Frobenius morphism descends to the minimal compactification, which is a standard argument adapted from Lan ([18]).

Theorem 1.1. Let $M_{n}$ be a (similitude) PEL Shimura variety with principal level $n$ structure, and $M_{n, \Sigma}^{\text {tor }}$ its toroidal compactification associated to an admissible smooth rational polyhedral cone decomposition $\Sigma$. We assume that $M_{n}$ is defined over a finite field over which we have a well-defined partial Frobenius map $F_{\mathfrak{p}_{i}}: M_{n} \rightarrow M_{n}$; then there is an admissible smooth rational polyhedral cone decomposition $\Sigma^{\prime}$ naturally associated to $\Sigma$ for which $F_{\mathfrak{p}_{i}}$ admits an extension

$$
F_{\mathfrak{p}_{i}}: M_{n, \Sigma}^{t o r} \longrightarrow M_{n, \Sigma^{\prime}}^{\text {tor }}
$$

\section{See Theorem 5.21 for a detailed description of $\Sigma^{\prime}$.}

Corollary 1.2. $F_{\mathfrak{p}_{i}}$ extends to the minimal compactification

$$
F_{\mathfrak{p}_{i}}: M_{n}^{\min } \longrightarrow M_{n}^{\min }
$$

Now the partial Frobenius acts on each summand of $E_{1}^{p, q}$, which, as we have already seen, is the intersection cohomology of (closure of) strata of the minimal compactifications with automorphic coefficients and have plectic Hodge structures given by $(\mathfrak{g}, K)$-cohomology. A subtle point here is that we have to pass to special fibres of integral models of Shimura varieties and use the spectral sequence 
(2) in the $l$-adic setting in order to have the action of the partial Frobenius and then compare it with the one in the Hodge theory setting. This can be done with some technical input from Huber and Morel's horizontal mixed complexes in [21] (a simpler proof in the special case of Hilbert modular varieties exists; see Remark 4.4).

Now, similar to the classical case, we have to check that the eigenvalues of the partial Frobenius on each summand are Weil numbers with absolute value compatible with the multi-weights of the plectic Hodge structures. In the case of Hilbert modular varieties, we have two different types of summands. The first type is when the summand is the cohomology of cusps with automorphic coefficients, which can be checked by direct computations.

The second is when it is the intersection cohomology of (minimal compactification of) Hilbert modular variety with trivial coefficients. This is decomposed into Hecke equivariant isotypic components indexed by discrete cohomological automorphic representations. If the automorphic representation is cuspidal, we know that it corresponds to a holomorphic Hilbert modular form $f$ of parallel weight 2, and the plectic Hodge type is

$$
\underset{v \mid \infty}{\otimes}((1,0) \oplus(0,1))
$$

which is of plectic weights $(1, \cdots, 1)$. We have to show that each partial Frobenius acts with eigenvalues of absolute value $p^{\frac{1}{2}}$. This follows from the Eichler-Shimura relation of the partial Frobenius proved by Nekovár in [25]. Indeed, it tells that the eigenvalues of the partial Frobenius are the same as the eigenvalues of the Frobenius $\operatorname{Frob}_{\mathfrak{p}} \in \operatorname{Gal}(\bar{F} / F)$ on the Galois representation $\rho_{f}$ associated to $f$, where $\mathfrak{p}$ ranges over primes of $F$ above $p$. We know that $\rho_{f}$ is pure of weight 1 by Blasius ([3]) and Blasius-Rogawski ([4]), proving the claim. If the automorphic representation is discrete but not cuspidal, we know that they are one-dimensional and have plectic Hodge types (wedge products of) the sum of $\underset{v \mid \infty}{\otimes}\left(p_{v}, q_{v}\right)$, with $\left(p_{v}, q_{v}\right)=(1,1)$ for one $v$ and $\left(p_{v}, q_{v}\right)=(0,0)$ for the rest, which is of plectic weight

$$
(0, \cdots, 0,2,0, \cdots, 0)
$$

This forces us to show that the partial Frobenius corresponding to $v$ (under the embedding $\mathbb{Q}_{p} \hookrightarrow \mathbb{C}$ implicitly fixed in the comparison theorem) has eigenvalues with absolute value $p$, and the rest have eigenvalues with absolute value 1 . This is shown by observing that these cohomology spaces are spanned by first Chern classes of the natural line bundles $L_{v}$ whose sections are modular forms of weight $(0, \cdots, 0,2,0, \cdots, 0)$, and the partial Frobenius acts on them in the expected way $\left(F_{v}^{*} L_{v}=L_{v}^{\otimes p}\right.$ and $\left.F_{v^{\prime}}^{*} L_{v}=L_{v}\right)$. Note that here we use a motivic explanation of the plectic strucutures to compare the plectic Frobenius weights and plectic Hodge weights, and this is the main reason we restrict to Hilbert modular varieties.

Now we have a $\mathbb{Z}^{d}$-filtration defined by eigenvalues of the partial Frobenius, and the last paragraph shows that the graded pieces have natural plectic Hodge structures given by $(\mathfrak{g}, K)$-cohomology in a compatible way. This finishes the construction of the plectic weight filtration and gives a conceptual explanation of the ad hoc construction of the plectic weight filtration by Nekovár and Scholl in [27]. Moreover, the proof has the potential to extend to more general situations where the naive construction of Nekovár and Scholl fails. Indeed, most ingredients we use are proved for general PEL-type Shimura varieties. The only serious obstacle for the general case is the use of motivic explanation as remarked above. To summarise, we have the following.

Theorem 1.3. Let $\mathscr{M}$ be a Hilbert modular variety, then there is an increasing $\mathbb{Z}^{d}$-filtration $W_{\underline{a}}$ (defined over $\mathbb{C})$ on $H^{*}(\mathscr{M}(\mathbb{C}), \mathbb{C})$ with $\underline{a}=\left(a_{1}, \cdots, a_{d}\right) \in \mathbb{Z}^{d}$, defined by

$$
W_{\underline{a}}=\bigoplus_{\substack{\beta_{i} \mid=p^{\frac{k_{i}}{2}} \\ k_{i} \leq a_{i}}} V_{\left(\beta_{1}, \cdots, \beta_{d}\right)},
$$


where $V_{\left(\beta_{1}, \cdots, \beta_{d}\right)}$ is the generalised eigenspace of $F_{i}$ with eigenvalue $\beta_{i}$ for all $i$. The action of $F_{i}$ on $H^{*}(\mathscr{M}(\mathbb{C}), \mathbb{C})$ is through the natural comparison isomorphism $H^{*}(\mathscr{M}(\mathbb{C}), \mathbb{C}) \cong l_{*} H^{*}\left(\mathscr{M}_{\overline{\mathbb{F}}_{p}}, \overline{\mathbb{Q}}_{l}\right)$ for some fixed isomorphism $\imath: \overline{\mathbb{Q}_{l}} \cong \mathbb{C}$.

The filtration is plectic in the sense that there is a natural plectic Hodge structure on $G r_{\underline{a}}^{W}$ with plectic weight $\underline{\text { a. }}$

The reader is warned that the construction does not a priori give the plectic mixed Hodge structure in the sense of Nekovár and Scholl ([27]) because we have not proved that the plectic Hodge filtration is compatible with plectic weight filtration. This is left to future works.

We now give a summary of each section. In Section 2 we review Morel's work on the weight $\mathrm{t}$-structures and prove a comparison theorem between two spectral sequences obtained using mixed Hodge modules and etale cohomology, respectively. In Section 3 we define the PEL moduli varieties and the partial Frobenius. This section is mostly to fix notations. In Section 4 we use the results on partial Frobenius proved in Section 5 and the weight spectral sequences in Section 2 to prove the existence of plectic weight structures on cohomology of Hilbert modular varieties. In particular, we make the weight spectral sequence in this case explicit in Subsection 4.2 and carry out the computations of the eigenvalues of the partial Frobenius in Subsection 4.3. Section 5 is a largely independent section, in which we prove that the partial Frobenius extends to toroidal compactifications and minimal compactifications. Following Lan, we review the construction of toroidal compactifications in Subsections 5.1 to 5.3. In particular, we review with some details on the degeneration data and how to construct it from degenerating abelian varieties. This is then used to construct the formal boundary strata of the toroidal compactifications and provides fundamental local formal models of the boundary strata. We use those constructions to prove the extension of partial Frobenius to toroidal and minimal compactifications in Subsections 5.4 and 5.5, respectively.

\section{Morel's weight t-structure}

\subsection{Formalism}

We review Morel's weight t-structures in this section. Everything in this section is due to Morel and Nair (the Hodge module case is due to Nair). The references we follow are [24] and [20].

In this section, $X$ denotes a separated scheme of finite type over a field $k$. We assume that $k$ is either finitely generated over its prime field or $k=\mathbb{C}$. Let $l$ be a prime number different from the characteristic of $k$ and $D_{c}^{b}\left(X, \bar{Q}_{l}\right)$ be the usual constructible derived category. We use $H^{i}$ to denote the cohomology with respect to the usual constructible t-structure and ${ }^{p} H^{i}$ for the cohomology with respect to the perverse t-structure. For Hodge modules, ${ }^{p} H^{i}$ will denote the usual cohomology of complexes of Hodge modules. They correspond to perverse cohomology under rat; see the paragraph after remark 2.1 for explanation of the terminology.

We denote both $D_{m}^{b}\left(X, \overline{\mathbb{Q}_{l}}\right)$ and $D^{b} M H M(X(\mathbb{C}))$ by $D_{m}^{b}(X)$, where $D_{m}^{b}\left(X, \overline{\mathbb{Q}_{l}}\right)$ is the bounded derived category of horizontal mixed complexes with weight filtrations as defined in [21] when $k$ is finitely generated, and $D^{b} M H M(X(\mathbb{C}))$ is the bounded derived category of Saito's mixed Hodge modules when $k=\mathbb{C}$. Note that here $m$ means 'mixed'. The key property of $D_{m}^{b}(X)$ is that they have the notion of weights and perverse t-structures, giving rise to canonical weight filtrations on perverse sheaves in $D_{m}^{b}(X)$. Further, morphisms between perverse sheaves strictly preserve weight filtrations. Under this abuse of notation, perverse sheaves refers to the usual perverse sheaves in the $l$-adic case and Hodge modules in the complex case.

When $k$ is a finite field, $D_{m}^{b}\left(X, \overline{\mathbb{Q}_{l}}\right)$ is the usual derived category of mixed sheaves defined by Deligne; that is, $D_{m}^{b}\left(X, \overline{\mathbb{Q}_{l}}\right) \subset D_{c}^{b}\left(X, \overline{Q_{l}}\right)$ is the full subcategory defined by $K \in D_{m}^{b}\left(X, \overline{\mathbb{Q}_{l}}\right)$ if and only if for every $i \in \mathbb{Z}, H^{i}(K)$ has a finite filtration $W$ whose graded pieces are pure in the sense that for every closed point $i_{x}: \operatorname{Spec}(k(x)) \hookrightarrow X$ and $n \in \mathbb{Z}, i_{x}^{*} G r_{n}^{W} H^{i}(K)$, as a representation of $\operatorname{Gal}(\bar{k} / k(x))$, has algebraic Frobenius eigenvalues whose absolute value are $(\# k(x))^{-n / 2}$ for every Archimedean place. 
We review Morel's construction in [21] of $D_{m}^{b}\left(X, \overline{Q_{l}}\right)$ for $k$ finitely generated. We can write $k$ as a direct limit of regular finite type $\mathbb{Z}$-algebras $A$ sitting inside $k$ and having fraction field $k$. The standard spreading argument shows that for $A$ as above (possibly passing to a localisation) there is a flat finite type $A$-scheme $\mathscr{X}_{A}$ such that $\left(\mathscr{X}_{A}\right)_{k} \cong X$. The $\left\{\mathscr{X}_{A}\right\}_{A}$ forms a direct system and induces natural functors between constructible derived categories. We define the derived category $D_{h}^{b}\left(X, \overline{\mathbb{Q}_{l}}\right)$ of horizontal constructible sheaves on $X$ to be the 2-limit of the category $D_{c}^{b}\left(\mathscr{X}_{A}, \overline{\mathbb{Q}_{l}}\right)$ indexed by $A$ as above. The perverse t-structures on $D_{c}^{b}\left(\mathscr{X}_{A}, \overline{\mathbb{Q}_{l}}\right)$ induce a t-structure on $D_{h}^{b}\left(X, \overline{\mathbb{Q}_{l}}\right)$, whose heart $\operatorname{Perv}_{h}(X)$ is called the category of horizontal perverse sheaves. The usual t-structures also induce a t-structure on $D_{h}^{b}\left(X, \overline{\mathbb{Q}_{l}}\right)$ whose hearts are called horizontal constructible sheaves. $K \in D_{h}^{b}\left(X, \overline{\mathbb{Q}_{l}}\right)$ is called mixed if $H^{i}(K)$ has a finite filtration whose graded pieces can be represented by a constructible sheaf $F_{A}$ on $\mathscr{X}_{A}$ such that for every closed point $x \in \operatorname{Spec}(A)$ (necessarily of finite residue field), $\left(F_{A}\right)_{x}$ is pure of some weight as discussed in the previous paragraph. Mixed horizontal complexes define a triangulated subcategory of $D_{h}^{b}\left(X, \overline{\mathbb{Q}_{l}}\right)$, and the perverse t-structure on $D_{h}^{b}\left(X, \overline{\mathbb{Q}_{l}}\right)$ induces a t-structure on it, whose heart $\operatorname{Perv}_{m}(X)$ is called the category of mixed horizontal perverse sheaves. The problem is that an element of $\operatorname{Perv}_{m}(X)$ does not necessarily have a weight filtration. However, the weight filtration is unique if it exists. We can define the subcategory $\operatorname{Perv}_{m f}(X)$ of $\operatorname{Perv}_{m}(X)$ consisting of those with a weight filtration. The uniqueness shows that morphisms in $\operatorname{Perv}_{m f}(X)$ are strict with respect to the weight filtration. Finally, we define the derived category of mixed horizontal perverse sheaves to be

$$
D_{m}^{b}\left(X, \overline{\mathbb{Q}_{l}}\right):=D^{b}\left(\operatorname{Perv}_{m f}(X)\right)
$$

Morel proved that the six functors can be defined on $D_{m}^{b}\left(X, \overline{\mathbb{Q}_{l}}\right)$. Note that for $k$ a finite field, $A=k$ and every mixed perverse sheaf has a weight filtration, proving that $D_{m}^{b}\left(X, \overline{\mathbb{Q}_{l}}\right)$ is identical to the category in the previous paragraph; see A. A. Ber̆linson, J. Bernstein and P. Deligne, 'Faisceaux pervers' ([2]) for details.

Remark 2.1. The constructions, especially the six functors, depend fundamentally on the finiteness results of Gabber; see [15]. If we restrict to $k$ with transcendental dimension smaller than 2, which is the only case we need, then the older finiteness results of Deligne in SGA [6] suffices. Moreover, Morel's proof uses sophisticated homological algebra results, including Beilinson's reconstruction of constructible t-structures from perverse ones, and Ayoub's work on crossed functors.

For mixed Hodge modules, we will not give a precise review. We only remind the reader that a mixed Hodge module consists of a good filtred regular holonomic $D$-module together with a perverse $\mathbb{Q}$-sheaf that is isomorphic after tensoring with $\mathbb{C}$ to the D-module under the Riemann-Hilbert correspondence. The precise conditions to put on these data are through a delicate induction process where vanishing cycles play an important role. It can be proved that admissible graded polarisable variations of Hodge structures are mixed Hodge modules, and they (their intermediate extension) constitute the simple mixed Hodge modules in a way similar to local systems and perverse sheaves. Forgetting about the $D$-modules gives a faithful functor

$$
\text { rat }: D^{b} \operatorname{MHM}(X) \rightarrow D_{c}^{b}(X, \mathbb{Q}),
$$

where we use the classical topology on $X(\mathbb{C})$ to define the right-hand side. An important property is that rat commutes with the six functors. The comparison theorem gives an $l$-adic perverse sheaf for each Hodge module. We will only use $\mathbb{C}$-Hodge modules, in which case the extra choices of perverse sheaves are redundant.

We will only need the cases when $k$ is a finite field, a number field or a complex number. Indeed, we will be primarily concerned with complex numbers, and finite fields come into play by reducing the complex situation to the finite fields cases. The reduction step will be achieved through number fields.

We now introduce Morel's fundamental weight t-structures. 
Definition/Theorem 2.2 ([20] Proposition 3.1.1). With notations as above, for $a \in \mathbb{Z} \cup\{\infty\}$ there is a $t$-structure

$$
\left({ }^{w} D^{\leq a},{ }^{w} D^{\geq a+1}\right)
$$

on $D_{m}^{b}(X)$ defined by $K \in{ }^{w} D^{\leq a}$ (respectively $K \in{ }^{w} D^{\geq a+1}$ ) if and only if for all $i \in \mathbb{Z},{ }^{p} H^{i}(K)$ has weights $\leq a$ (respectively $\geq a+1)$. Moreover, ${ }^{w} D^{\leq a}$ and ${ }^{w} D^{\geq a+1}$ are triangulated subcategories and are stable under extensions. For $K \in{ }^{w} D^{\leq a}$ and $L \in{ }^{w} D^{\geq a+1}$, we have

$$
\operatorname{RHom}(K, L)=0 .
$$

Note that this is stronger than being given by a $t$-structure. We have ${ }^{w} D^{\leq a}(1)={ }^{w} D^{\leq a-2}$ and ${ }^{w} D^{\geq a}(1)=$ ${ }^{w} D^{\geq a-2}$, where (1) is the Tate twist.

Remark 2.3. The t-structure is unusual in that it has trivial heart and is stable under shift [1] in the triangulated category. Note that a complex $K \in{ }^{w} D^{\leq a} \cap{ }^{w} D^{\geq a}$ is not a pure complex of weight $a$ in the sense of Deligne, which means $H^{i}(K)$ that has weight $a+i$ (or, equivalently, ${ }^{p} H^{i}(K)$ has weight $\mathrm{i}+\mathrm{a}$ ).

Recall that (over finite fields) a pure complex is a direct sum of its shifted perverse cohomology after base change to the algebraic closure, and the decomposition does not hold before base change. This fact plays an important role in the proof of the decomposition theorem. The next proposition gives a variant of this fact in complete generality. In particular, we do not need to pass to algebraic closure.

Proposition 2.4 ([24] Lemma 2.2.3). If $K \in{ }^{w} D^{\geq a} \cap{ }^{w} D^{\leq a}$, we have an isomorphism

$$
K \cong \underset{i}{\oplus} H^{i}(K)[-i]
$$

The constituents ${ }^{p} H^{i}(K)$ are pure, and they decompose by supports into intersection complexes; that is, intermediate extension of smooth sheaves on a smooth locally closed subscheme.

Moreover, this isomorphism is canonical and the constituents are semisimple if we are in the mixed Hodge modules case.

Remark 2.5. The corresponding statement is not true in the $l$-adic case.

The t-structure gives us functors $w_{\leq a}: D_{m}^{b}(X) \rightarrow{ }^{w} D^{\leq a}$ (respectively $w_{\geq a}: D_{m}^{b}(X) \rightarrow{ }^{w} D^{\geq a}$ ) such that for every $K \in D_{m}^{b}(X)$, we have a distinguished triangle

$$
w_{\leq a} K \longrightarrow K \longrightarrow w_{\geq a+1} K \stackrel{+1}{\longrightarrow} .
$$

If $K$ is written as a complex of perverse sheaves $K_{i}$, which is always possible, then $w_{\leq a} K$ is the complex represented by $w_{\leq a} K_{i}$, where $w_{\leq a} K_{i}$ is the weight filtration on $K_{i}$. We have the following proposition on the behaviour of $w_{\leq a}$.

Proposition 2.6 ([20] Proposition 3.1.3). Let $K \in D_{m}^{b}(X)$, then we have that $w_{\leq a}$ (respectively $w_{\geq a}$ ) is exact with respect to the perverse $t$-structure; that is,

$$
\begin{gathered}
w_{\leq a}{ }^{p} H^{i}(K)={ }^{p} H^{i}\left(w_{\leq a} K\right) \\
w_{\geq a}{ }^{p} H^{i}(K)={ }^{p} H^{i}\left(w_{\geq a} K\right) .
\end{gathered}
$$

Moreover, the distinguished triangle

$$
w_{\leq a} K \longrightarrow K \longrightarrow w_{\geq a+1} K \stackrel{+1}{\longrightarrow} .
$$


induces a short exact sequence of perverse sheaves

$$
0 \longrightarrow{ }^{p} H^{i}\left(w_{\leq a} K\right) \longrightarrow{ }^{p} H^{i}(K) \longrightarrow{ }^{p} H^{i}\left(w_{\geq a+1} K\right) \longrightarrow 0 .
$$

The four functors interact with the weight $\mathrm{t}$-structure as described in the following proposition.

Proposition 2.7 ([20] Proposition 3.1.3). Let $f: X \rightarrow Y$ be a morphism with dimension of the fibres less than or equal to $d$, then

$$
\begin{aligned}
& R f_{!}\left({ }^{w} D^{\leq a}(X)\right) \subset{ }^{w} D^{\leq a+d}(Y) \\
& f^{*}\left({ }^{w} D^{\leq a}(Y)\right) \subset{ }^{w} D^{\leq a+d}(X) \\
& R f_{*}\left({ }^{w} D^{\geq a}(X)\right) \subset{ }^{w} D^{\geq a-d}(Y) \\
& f^{!}\left({ }^{w} D^{\geq a}(Y)\right) \subset{ }^{w} D^{\geq a-d}(X) .
\end{aligned}
$$

The duality functor $D:=R \operatorname{Hom}\left(-, \omega_{X}\right)\left(\omega_{X}\right.$ is the dualising complex) exchanges ${ }^{w} D^{\leq a}(X)$ and ${ }^{w} D^{\geq-a}(X)$; that is, $D\left({ }^{w} D^{\leq a}(X)\right)={ }^{w} D^{\geq-a}(X)$, so

$$
D \circ w_{\leq a}=w_{\geq-a} \circ D .
$$

The most important property of $w_{\leq a}$ is its relation with the intermediate extension functor.

Theorem 2.8 ([20] Theorem 3.1.4). Let $j: U \rightarrow X$ be a nonempty open embedding and $K \in D_{m}^{b}(X)$ a pure perverse sheaf of weight a on $U$; then we have natural isomorphisms

$$
w_{\geq a} j_{!} K=j_{! *} K=w_{\leq a} R j_{*} K .
$$

We now introduce a refined version of the weight t-structure, taking a specified stratification into consideration. Let $X=\underset{0 \leq i \leq n}{\cup} S_{i}$ be a stratification such that each $S_{i}$ is locally closed in $X$ and $S_{k}$ is open in $\underset{k \leq i \leq n}{\cup} S_{i}$ for every $k \in[0, n]$. Let $\underline{a}=\left(a_{0}, \cdots, a_{n}\right)$ with each $a_{i} \in \mathbb{Z} \cup\{\infty\}$ and $i_{k}: S_{k} \hookrightarrow X$ be the inclusion.

Definition/Theorem 2.9 ([20] Proposition 3.3.2). Let $^{w} D^{\leq} \underline{a}$ (respectively ${ }^{w} D^{\geq} \underline{a}$ ) be the subcategory of $D_{m}^{b}(X)$ defined by $K \in{ }^{w} D^{\leq \underline{a}}$ (respectively $K \in{ }^{w} D^{\geq}$) if and only if $i_{k}^{*} K \in{ }^{w} D^{\leq a_{k}}\left(S_{k}\right)$ (respectively $\left.i_{k}^{!} K \in{ }^{w} D^{\geq a_{k}}\left(S_{k}\right)\right)$ for every $k$. Then

$$
\left({ }^{w} D^{\leq \underline{a}},{ }^{w} D^{\geq \underline{a}+\underline{1}}\right)
$$

defines a $t$-structure on $D_{m}^{b}(X)$, giving rise to functors

$$
w_{\leq \underline{a}}: D_{m}^{b}(X) \rightarrow{ }^{w} D^{\leq \underline{a}}
$$

and

$$
w_{\geq \underline{a}}: D_{m}^{b}(X) \rightarrow{ }^{w} D^{\geq \underline{a}}
$$

such that for every $K \in D_{m}^{b}(X)$, there is a distinguished triangle

$$
w_{\leq \underline{a}} K \longrightarrow K \longrightarrow w_{\geq \underline{a}+\underline{1}} K \stackrel{+1}{\longrightarrow} .
$$

Moreover, we have RHom $(L, K)=0$ for $L \in{ }^{w} D^{\leq \underline{a}}$ and $K \in{ }^{w} D^{\geq \underline{a}+\underline{1}}$.

Most of the properties of ${ }^{w} D^{\leq a}$ (respectively ${ }^{w} D^{\geq a}$ ) generalise to ${ }^{w} D^{\leq} \underline{a}$ (respectively ${ }^{w} D^{\geq} \underline{a}$ ). We summarise them as follows. 
Theorem 2.10 ([20] Proposition 3.4.1). ${ }^{w} D^{\leq \underline{a}}$ and ${ }^{w} D^{\geq \underline{a}}$ are triangulated subcategories of $D_{m}^{b}(X)$ that are stable under extensions. If $\underline{a}=(a, \cdots, a)$, then ${ }^{w} D^{\geq} \underline{a}={ }^{w} D^{\geq a}$ and ${ }^{w} D^{\leq \underline{a}}={ }^{w} D^{\leq a}$.

For $Y$ another scheme with strata $\left\{S_{i}^{\prime}\right\}_{0 \leq i \leq n}$ satisfying the same condition as before, and $f: X \rightarrow Y$ a morphism such that $f\left(S_{k}\right) \subset S_{k}^{\prime}$, assume that the dimension of the fibres of $f$ is smaller than or equal to $d$. Then we have

$$
\begin{aligned}
& R f_{!}\left({ }^{w} D^{\leq \underline{a}}(X)\right) \subset{ }^{w} D^{\leq \underline{a}+\underline{d}}(Y) \\
& f^{*}\left({ }^{w} D^{\leq \underline{a}}(Y)\right) \subset{ }^{w} D^{\leq \underline{a}+\underline{d}}(X) \\
& R f_{*}\left({ }^{w} D^{\geq \underline{a}}(X)\right) \subset{ }^{w} D^{\geq \underline{a}-\underline{d}}(Y) \\
& f^{!}\left({ }^{w} D^{\geq \underline{a}}(Y)\right) \subset{ }^{w} D^{\geq \underline{a}-\underline{d}}(X) .
\end{aligned}
$$

Further, we have

$$
D \circ w_{\leq \underline{a}}=w_{\geq-\underline{a}} \circ D .
$$

The next proposition tells us how to compute $w_{\leq \underline{a}}$ and $w_{\geq \underline{a}}$ in terms of $w_{\leq a}$ and $w_{\geq a}$.

Proposition 2.11 ([20] Proposition 3.3.4). Let $k \in\{0 \cdots n\}$ and $a \in \mathbb{Z} \cup\{\infty\}$, then we denote

$$
\begin{aligned}
w_{\leq a}^{k} & :=w_{\leq(\infty, \cdots, \infty, a, \infty, \cdots, \infty)} \\
w_{\geq a}^{k} & :=w_{\geq(\infty, \cdots, \infty, a, \infty, \cdots, \infty),}
\end{aligned}
$$

where a sits in the kth position. We have

$$
\begin{aligned}
& w_{\leq \underline{a}}=w_{\leq a_{n}}^{n} \circ \cdots \circ w_{\leq a_{0}}^{0} \\
& w_{\geq \underline{a}}=w_{\geq a_{n}}^{n} \circ \cdots \circ w_{\geq a_{0}}^{0} .
\end{aligned}
$$

For $K \in D_{m}^{b}(X)$, we have distinguished triangles

$$
\begin{gathered}
w_{\leq a}^{k} K \longrightarrow K \longrightarrow R i_{k *} w_{\geq a+1} i_{k}^{*} K \stackrel{+1}{\longrightarrow} . \\
i_{k !} w_{\leq a-1} i_{k}^{!} K \longrightarrow K \longrightarrow w_{\geq a}^{k} K \stackrel{+1}{\longrightarrow} .
\end{gathered}
$$

Corollary 2.12. We have natural isomorphisms

$$
\begin{aligned}
& i_{k}^{*} \circ w_{\leq a}^{k}=w_{\leq a} \circ i_{k}^{*} \\
& i_{k}^{!} \circ w_{\geq a}^{k}=w_{\geq a} \circ i_{k}^{!}
\end{aligned}
$$

and

$$
\begin{aligned}
& i_{j}^{*} \circ w_{\leq a}^{k}=i_{j}^{*} \\
& i_{j}^{!} \circ w_{\geq a}^{k}=i_{j}^{!}
\end{aligned}
$$

for $j<k$.

Theorem 2.13 ([20] Proposition 3.4.2). Let $U:=S_{0}$ and $j=i_{0}: U \hookrightarrow X$ be the inclusion of the open stratum, then for $K \in D_{m}^{b}(U)$ a pure perverse sheaf of weight a we have

$$
w_{\geq(a, a+1, \cdots, a+1)} j_{!} K=j_{! *} K=w_{\leq(a, a-1, \cdots, a-1)} R j_{*} K .
$$




\subsection{Applications to Shimura varieties}

In this subsection, we take $X$ to be a Shimura variety associated to a Shimura datum $(G, \mathscr{X})$, where $G$ is a reductive group over $\mathbb{Q}$ and $\mathscr{X}$ is a conjugacy class of cocharacters $\operatorname{Res}_{\mathbb{C} / \mathbb{R}} \mathbf{G}_{m} \longrightarrow G_{\mathbb{R}}$. The pair has to satisfy a list of axioms to be a Shimura datum, which we will not review. We assume that $X$ is smooth, which can always be achieved if we take a small enough level structure. An important property of Shimura varieties is that they have a canonical model over a number field $F$, called the reflex field of $(G, \mathscr{X})$. For simplicity, we assume that $G$ is simple.

An algebraic rational representation of $G$ naturally gives an admissible variation of Hodge structure on $X$, whence a mixed Hodge module. The representation creates a smooth $l$-adic sheaf on $X$ as well. However, unlike Hodge modules, the $l$-adic sheaf is not known to be mixed in general, although this is expected to be the case. Fortunately, we know that the associated $l$-adic sheaves are of geometric origin, and hence mixed, if the Shimura variety is of abelian type. We will only need to work with Shimura varieties of PEL type (up to similitude) in this article, so we make this assumption from now on. We note that PEL-type Shimura varieties have the hereditary property that strata of the minimal compactification are also of PEL type.

Let $X^{\mathrm{min}}$ be the minimal compactification of $X$. It has a natural stratification $X^{\mathrm{min}}=\underset{0 \leq i \leq n}{\cup} S_{i}$ with $S_{0}=X$ and $S_{k}$ open in $\underset{k \leq i \leq n}{\cup} S_{i}$ for each $k$. Each $S_{i}$ is the union of standard strata corresponding to parabolic subgroups of $G$ of a fixed type. We will not give an explicit description of $S_{i}$ here; see Nair [24] for details.

Let $V$ be a rational algebraic representation of $G$ and $\mathcal{F} V \in D_{m}^{b}(X)$ the corresponding sheaf. We note that $\mathcal{F} V$ is concentrated in degree 0 and smooth. Let $j: X \hookrightarrow X^{\mathrm{min}}$ be the open embedding, then applying $R \Gamma\left(X^{\mathrm{min}},-\right)$ to the weight truncations $w_{\leq a} R j_{*}(\mathcal{F} V)$ of $R j_{*}(\mathcal{F} V)$ induces a spectral sequence

$$
E_{1}^{p, q}=H^{p+q}\left(X^{\min }, w_{\geq-p} w_{\leq-p} R j_{*}(\mathcal{F} V)\right) \Rightarrow H^{p+q}(X, \mathcal{F} V)
$$

Because $G$ is simple reductive, we can assume that $V$ is irreducible and pure of weight $-a$. Note that the weight of $V$ is the weight of the representation $\mathbf{G}_{m \mathbb{R}} \hookrightarrow \operatorname{Res}_{\mathbb{C} / \mathbb{R}} \mathbf{G}_{m} \stackrel{h}{\rightarrow} G_{\mathbb{R}} \rightarrow \operatorname{End}\left(V_{\mathbb{R}}\right)$ for one (and hence any) $h \in \mathscr{X}$. Then $\mathcal{F} V$ is pure of weight $a$, and the first nontrivial truncation of $R j_{*}(\mathcal{F} V)$ ) is

$$
w_{\geq a} w_{\leq a} R j_{*}(\mathcal{F} V)=w_{\leq a} R j_{*}(\mathcal{F} V)=j_{! *}(\mathcal{F} V)
$$

by Proposition 2.7 and Theorem 2.8. It completes into a distinguished triangle

$$
j_{! *}(\mathcal{F} V) \longrightarrow R j_{*}(\mathcal{F} V) \longrightarrow w_{\geq a+1} R j_{*}(\mathcal{F} V) \stackrel{+1}{\longrightarrow} .
$$

which shows that $w_{\geq a+1} R j_{*}(\mathcal{F} V)$ has support in the complement of $X$ as $j^{*} j_{\text {!* }}=j^{*} R j_{*}=i d$. Let $i: \underset{1 \leq i \leq n}{\cup} S_{i} \hookrightarrow X^{\mathrm{min}}$ be the complement of $X$, then

$$
{ }^{w} D^{\geq a+1} \ni w_{\geq a+1} R j_{*}(\mathcal{F} V)=i_{*}\left(i^{*} w_{\geq a+1} R j_{*}(\mathcal{F} V)\right)
$$

Because $i_{*}=i_{\text {! }}$ is exact with respect to the weight $t$-structure by Proposition $2.7, i^{*} w_{\geq a+1} R j_{*}(\mathcal{F} V) \in$ ${ }^{w} D^{\geq a+1}$. Applying $i^{*}$ to the distinguished triangle

$$
w_{\leq a} R j_{*}(\mathcal{F} V) \longrightarrow R j_{*}(\mathcal{F} V) \longrightarrow w_{\geq a+1} R j_{*}(\mathcal{F} V) \stackrel{+1}{\longrightarrow} .
$$

we have

$$
i^{*} w_{\leq a} R j_{*}(\mathcal{F} V) \longrightarrow i^{*} R j_{*}(\mathcal{F} V) \longrightarrow i^{*} w_{\geq a+1} R j_{*}(\mathcal{F V}) \stackrel{+1}{\longrightarrow} \cdot
$$


We see by Proposition 2.7 that $i^{*} w_{\leq a} R j_{*}(\mathcal{F} V) \in{ }^{w} D^{\leq a}$. Together with $i^{*} w_{\geq a+1} R j_{*}(\mathcal{F V}) \in{ }^{w} D^{\geq a+1}$ that we have just observed, we obtain

$$
i^{*} w_{\geq a+1} R j_{*}(\mathcal{F} V)=w_{\geq a+1} i^{*} R j_{*}(\mathcal{F} V) .
$$

Therefore,

$$
w_{\leq a+1} w_{\geq a+1} R j_{*}(\mathcal{F} V)=w_{\leq a+1} i_{*} w_{\geq a+1} i^{*} R j_{*}(\mathcal{F} V)=i_{*} w_{\leq a+1} w_{\geq a+1} i^{*} R j_{*}(\mathcal{F} V)
$$

and, similarly,

$$
w_{\leq a+k} w_{\geq a+k} R j_{*}(\mathcal{F} V)=i_{*} w_{\leq a+k} w_{\geq a+k} i^{*} R j_{*}(\mathcal{F} V)
$$

for all $k>0$ (applying $w_{\leq a+k} w_{\geq a+k}$ to $w_{\geq a+1} R j_{*}(\mathcal{F} V)$ and using that $w_{\geq a+k} w_{\geq a+1}=w_{\geq a+k}$ ).

It is shown that $i^{*} R j_{*}(\mathcal{F} V)$ is constructible with respect to the standard stratification (and in particular for $\left\{S_{i}\right\}$ ) by Burgos and Wildeshaus ([5]) in the Hodge Module case and Pink ([29]) in the $l$-adic case. Moreover, the restriction of $i^{*} R j_{*}(\mathcal{F} V)$ to strata has automorphic cohomology sheaves in the sense that they are associated to algebraic representations of the group corresponding to the strata as a Shimura variety. We claim that $w_{\leq a+k} w_{\geq a+k} i^{*} R j_{*}(\mathcal{F V})$ is also constructible with respect to the standard stratification and even automorphic when restricted to each stratum. Indeed, by Proposition 2.11,

$$
w_{\leq a+k} i^{*} R j_{*}(\mathcal{F} V)=w_{\leq(a+k, \cdots, a+k)} i^{*} R j_{*}(\mathcal{F} V)=w_{\leq a+k}^{n} \circ \cdots \circ w_{\leq a+k}^{1} i^{*} R j_{*}(\mathcal{F} V)
$$

and there is a distinguished triangle

$$
w_{\leq a+k}^{1} i^{*} R j_{*}(\mathcal{F} V) \longrightarrow i^{*} R j_{*}(\mathcal{F} V) \longrightarrow i_{1 *} w_{\geq a+k+1} i_{1}^{*} i^{*} R j_{*}(\mathcal{F} V) \stackrel{+1}{\longrightarrow} .
$$

Because both $i^{*} R j_{*}(\mathcal{F V})$ and $i_{1 *} w_{\geq a+k+1} i_{1}^{*} i^{*} R j_{*}(\mathcal{F V})$ are constructible and automorphic with respect to the standard stratification (using $w_{\leq a} \mathcal{F} V=\mathcal{F}\left(w_{\geq \operatorname{dim} X-a} V\right)$; see [20] Lemma 4.1.2), so is $w_{\leq a+k}^{1} i^{*} R j_{*}(\mathcal{F} V)$. The same argument applies to $w_{\leq a+k}^{2}$ by replacing $i^{*} R j_{*}(\mathcal{F} V)$ to $w_{\leq a+k}^{1} i^{*} R j_{*}(\mathcal{F V})$, and an easy induction proves that $w_{\leq a+k} i^{*} R j_{*}(\mathcal{F} V)$ is constructible and automorphic. The claim follows from the distinguished triangle

$$
w_{\leq a+k-1} i^{*} R j_{*}(\mathcal{F} V) \longrightarrow w_{\leq a+k} i^{*} R j_{*}(\mathcal{F} V) \longrightarrow w_{\geq a+k} w_{\leq a+k} i^{*} R j_{*}(\mathcal{F} V) \stackrel{+1}{\longrightarrow} .
$$

and what we have just proved for the first two terms. Note that $w_{\geq a+k} w_{\leq a+k}=w_{\leq a+k} w_{\geq a+k}$ (see [24] Lemma 2.2.3).

Recall that Proposition 2.4 tells us that $w_{\geq a+k} w_{\leq a+k} i^{*} R j_{*}(\mathcal{F} V)$ decompose into shifts of pure perverse sheaves, and the claim we have just proved shows that these perverse sheaves are intermediate extensions of automorphic sheaves on the standard strata. This is also true for $w_{\geq a+k} w_{\leq a+k} R j_{*}(\mathcal{F} V)$ using (3) and (4). We know that the normalisation of the closure of a stratum is the minimal compactification of the stratum and intersection cohomology is invariant under normalisation; hence, $\mathbb{H}\left[{ }^{p+q}\left(X^{\mathrm{min}}, w_{\geq-p} w_{\leq-p} R j_{*}(\mathcal{F} V)\right)\right.$ is a sum of intersection cohomology of the minimal compactification of the strata with coefficients automorphic sheaves. We now summarise what we have proved.

Theorem 2.14 (Nair [24]). For X a Shimura variety of PEL type with Shimura data $(G, X)$ and $V$ a representation of $G$, we have a spectral sequence

$$
E_{1}^{p, q}=\mathbb{H}^{p+q}\left(X^{m i n}, w_{\geq-p} w_{\leq-p} R j_{*}(\mathcal{F} V)\right) \Rightarrow H^{p+q}(X, \mathcal{F} V)
$$

where $\mathbb{H}^{p+q}\left(X^{\min }, w_{\geq-p} w_{\leq-p} R j_{*}(\mathcal{F} V)\right)$ is a sum of $I H^{*}\left(Y^{\min }, \mathcal{F} W\right):=\mathbb{H}^{*}\left(Y^{\text {min }}, j_{! *} \mathcal{F} W\right)$ with $Y \subset X^{\text {min }}$ a standard strata and $W$ an algebraic representation of the group associated to $Y$.

Remark 2.15. It is possible to write $E_{1}^{p, q}$ more explicitly using Pink's ([29]) or Burgos and Wildeshaus's ([5]) results. We will do that with Hilbert modular varieties later. 
We know that the PEL-type Shimura variety $X$ has a natural smooth integral model $\mathfrak{X}$ over an open subset $\mathcal{U}$ of $\operatorname{Spec}_{F}$ and the automorphic sheaf $\mathcal{F} V$ extends to $\mathfrak{X}$, which we still denote by $\mathcal{F} V$. Let $\operatorname{Spec}(k)$ be a closed point of $\mathcal{U}$; hence $k$ is a finite field. The above theorem 2.14 gives us two spectral sequences

$$
{ }_{H} E_{1}^{p, q}=\mathbb{H}^{p+q}\left(X^{\min }(\mathbb{C}), w_{\geq-p} w_{\leq-p} R j_{*}\left({ }_{H} \mathcal{F} V\right)\right) \Rightarrow H^{p+q}\left(X(\mathbb{C}),{ }_{H} \mathcal{F} V\right)
$$

and

$$
{ }_{l} E_{1}^{p, q}=\mathbb{H}^{p+q}\left(\mathfrak{X}_{\bar{k}}^{\min }, w_{\geq-p} w_{\leq-p} R j_{*}\left({ }_{l} \mathcal{F} V\right)\right) \Rightarrow H^{p+q}\left(\mathfrak{X}_{\bar{k}},{ }_{l} \mathcal{F} V\right),
$$

where ${ }_{H} \mathcal{F} V$ is the $\left(\mathbb{C}\right.$-) Hodge module associated to $V$ (it is normalised so that $\left.\operatorname{rat}{ }_{H} \mathcal{F} V\right)=\mathcal{F} V[0] \in$ $D_{c}^{b}(X(\mathbb{C}), \mathbb{Q})$; in other words, ${ }_{H} \mathcal{F} V \in D^{b} M H M(X(\mathbb{C}))$ sit in degree $\left.\operatorname{dim} X\right)$ in the first spectral sequence and ${ }_{H} E_{1}^{p, q}$ is obtained from the weight truncation in $D^{b} M H M(X(\mathbb{C}))$. Similarly, ${ }_{l} \mathcal{F} V$ is the mixed $l$-adic lisse sheaf associated to $V$ in the second one, and the spectral sequence is obtained by looking at the weight truncation in $D_{m}^{b}\left(\mathfrak{X}_{k}, \overline{\mathbb{Q}_{l}}\right)$ and then passing to the algebraic closure of $k$. Note that the first spectral sequence takes values in (complex) mixed Hodge structures, and the second takes values in $\operatorname{Gal}(\bar{k} / k)$-modules. The next theorem provides a comparison between the two spectral sequences. Because it seems not to be in the literature, we give a proof.

Theorem 2.16. Fix an isomorphism $\iota: \mathbb{C} \cong \overline{\mathbb{Q}_{l}}$, then for all but finitely many $\operatorname{Spec}(k) \subset \mathcal{U}$ there is $a$ natural isomorphism

$$
l_{*} H^{n}\left(X(\mathbb{C}),{ }_{H} \mathcal{F} V\right) \cong H^{n}\left(\mathfrak{X}_{\bar{k}}, l_{l} \mathcal{F} V\right)
$$

as $\overline{\mathbb{Q}_{l}}$-vector spaces, and the filtrations induced by ${ }_{H} E_{1}^{p, q}$ and ${ }_{l} E_{1}^{p, q}$ are identified through the isomorphism.

Proof. Recall that $D_{m}^{b}\left(X / F, \overline{\mathbb{Q}_{l}}\right)$ is the derived category of horizontal mixed complexes on $X / F$, which is defined by the direct limit of suitable subcategories of $D_{c}^{b}\left(\mathfrak{X}_{\mathcal{V}}, \overline{\mathbb{Q}_{l}}\right)$, indexed by open subsets $\mathcal{U} \subset \mathcal{U}$. Because $_{l} \mathcal{F V}$ extends to $\mathfrak{X}$, it defines an element ${ }_{l} \mathcal{F} V \in D_{m}^{b}\left(X / F, \overline{\mathbb{Q}_{l}}\right)$. Because $X^{\text {min }}$ also descends to a canonical model $\mathfrak{X}^{\text {min }}$ over $\mathcal{U}$, which is a compactification of $\mathfrak{X}$, we have that $R j_{*}\left({ }_{l} \mathcal{F} V\right) \in D_{c}^{b}\left(\mathfrak{X}^{\text {min }}, \overline{\mathbb{Q}_{l}}\right)$ defines an element of $D_{m}^{b}\left(X^{\mathrm{min}} / F, \overline{\mathbb{Q}_{l}}\right)$.

The weight t-structure on $D_{m}^{b}\left(X^{\mathrm{min}} / F, \overline{\mathbb{Q}_{l}}\right)$ gives the truncations $w_{\leq a} R j_{*}\left({ }_{l} \mathcal{F} V\right) \in D_{m}^{b}\left(X^{\mathrm{min}} / F, \overline{\mathbb{Q}_{l}}\right)$, which are represented by complexes on $\mathfrak{X}_{\mathcal{V}}^{\text {min }}$ for some nonempty open subset $\mathcal{V} \subset \mathcal{U}$ by definition of the horizontal complexes. Because there are only finitely many truncations, we can assume that $\mathcal{V}$ is chosen such that all of the truncations are represented by complexes on $\mathfrak{X}_{\mathcal{V}}^{\min }$, which we still denote by $w_{\leq a} R j_{*}\left({ }_{l} \mathcal{F} V\right) \in D_{c}^{b}\left(\mathfrak{X}_{\mathcal{V}}^{\min }, \overline{\mathbb{Q}_{l}}\right)$.

Recall that weights on $D_{m}^{b}\left(X^{\mathrm{min}} / F, \overline{\mathbb{Q}_{l}}\right)$ are defined by first reducing to finite fields and then taking the weights there. We have basically from the definition that

$$
\left(w_{\leq a} R j_{*}\left({ }_{l} \mathcal{F} V\right)\right)_{k}=w_{\leq a}\left(\left(R j_{*}\left({ }_{l} \mathcal{F} V\right)\right)_{k}\right) \in D_{m}^{b}\left(\mathfrak{X}_{k}^{\min }, \overline{\mathbb{Q}_{l}}\right) .
$$

By the lemma 2.17 below, we have $\left(R j_{*}(l \mathcal{F} V)\right)_{k}=R j_{k *}\left(\left.l \mathcal{F} V\right|_{\mathfrak{X}_{k}}\right)$, where $j_{k}: \mathfrak{X}_{k} \hookrightarrow \mathfrak{X}_{k}^{\text {min }}$ is the base change of $j$ to $k$. Thus, we have

$$
\left(w_{\leq a} R j_{*}\left({ }_{l} \mathcal{F} V\right)\right)_{k}=w_{\leq a} R j_{k *}\left(\left.l \mathfrak{F} V\right|_{\mathfrak{x}_{k}}\right) .
$$

We base change $w_{\leq a} R j_{*}\left({ }_{l} \mathcal{F} V\right) \in D_{c}^{b}\left(\mathfrak{X}_{\mathcal{V}}^{\min }, \overline{\mathbb{Q}_{l}}\right)$ to a complex point of $\mathcal{V}$; then the comparison between etale and classical sites and that $\mathcal{F} V$ is of geometric origin provide us with a natural isomorphism

$$
\left(w_{\leq a} R j_{*}\left({ }_{l} \mathcal{F} V\right)\right)_{\mathbb{C}} \cong l_{*} \operatorname{rat}\left(w_{\leq a} R j_{\mathbb{C} *}\left({ }_{H} \mathcal{F} V\right)\right) .
$$


Let $\mathcal{V}_{(k)}$ be the etale localisation of $\mathcal{V}$ at $\operatorname{spec}(k)$ and $\bar{\eta}$ the geometric generic point of $\mathcal{V}_{(k)}$. By properness of $g: \mathfrak{X}_{\mathcal{V}_{(k)}}^{\min } \rightarrow \mathcal{V}_{(k)}$, we have

$$
\begin{gathered}
R \Gamma\left(\mathfrak{X}_{\bar{\eta}}^{\min },\left(w_{\leq a} R j_{*}\left({ }_{l} \mathcal{F} V\right)\right)_{\bar{\eta}}\right)=R \Gamma\left(\bar{\eta},\left(R g_{*} w_{\leq a} R j_{*}\left({ }_{l} \mathcal{F} V\right)\right)_{\bar{\eta}}\right)=R \Gamma\left(\mathcal{V}_{(k)}, R g_{*} w_{\leq a} R j_{*}\left({ }_{l} \mathcal{F} V\right)\right) \\
=\left(R g_{*} w_{\leq a} R j_{*}\left({ }_{l} \mathcal{F} V\right)\right)_{\bar{k}}=R \Gamma\left(\mathfrak{X}_{\bar{k}}^{\min },\left(w_{\leq a} R j_{*}\left({ }_{l} \mathcal{F} V\right)\right)_{\bar{k}}\right) .
\end{gathered}
$$

Together with equation (5), we have

$$
R \Gamma\left(\mathfrak{X}_{\bar{\eta}}^{\min },\left(w_{\leq a} R j_{*}\left({ }_{l} \mathcal{F} V\right)\right)_{\bar{\eta}}\right)=R \Gamma\left(\mathfrak{X}_{\bar{k}}^{\min }, w_{\leq a} R j_{k *}\left(\left.{ }_{l} \mathcal{F} V\right|_{\mathfrak{X}_{k}}\right)\right) .
$$

Choose an embedding of $\bar{\eta}$ into $\mathbb{C}$, then (6) and (7) give us

$$
\begin{aligned}
& l_{*} H^{n}\left(X^{\min }(\mathbb{C}), w_{\leq a} R j_{\mathbb{C} *}(H \mathcal{F} V)\right) \cong H^{n}\left(X_{\mathbb{C}}^{\min },\left(w_{\leq a} R j_{*}\left({ }_{l} \mathcal{F} V\right)\right)_{\mathbb{C}}\right) \\
& =H^{n}\left(\mathfrak{X}_{\bar{\eta}}^{\min },\left(w_{\leq a} R j_{*}\left({ }_{l} \mathcal{F} V\right)\right)_{\bar{\eta}}\right)=H^{n}\left(\mathfrak{X}_{\bar{k}}^{\min }, w_{\leq a} R j_{k *}\left(\left.{ }_{l} \mathcal{F} V\right|_{\mathfrak{x}_{k}}\right)\right) .
\end{aligned}
$$

We know by definition of the spectral sequence ${ }_{H} E_{1}^{p, q}$ that the image of $l_{*} H^{n}\left(X^{\min }(\mathbb{C}), w_{\leq a}\right.$ $\left.R j_{\mathbb{C}_{*}}\left({ }_{H} \mathcal{F} V\right)\right)$ in

$$
l_{*} H^{n}\left(X^{\min }(\mathbb{C}), R j_{\mathbb{C} *}\left({ }_{H} \mathcal{F} V\right)\right)=l_{*} H^{n}\left(X(\mathbb{C}),{ }_{H} \mathcal{F} V\right)
$$

is the filtration corresponding to ${ }_{H} E_{1}^{p, q}$, and similarly for $H^{n}\left(\mathfrak{X}_{\bar{k}}^{\min }, w_{\leq a} R j_{k *}\left({ }_{l} \mathcal{F} V \mid \mathfrak{x}_{k}\right)\right)$. The isomorphism (8) for $a$ large enough defines the isomorphism in the statement of the theorem, and it respects the filtration by what we have just observed.

Lemma 2.17. Let $j: \mathfrak{X} \hookrightarrow \mathfrak{X}^{\text {min }}$ be the inclusion of a PEL Shimura variety into its minimal compactification, defined over $\mathcal{U} \subset \operatorname{Spec}\left(\mathcal{O}_{F}\right)$, and $V$ an algebraic representation of the group $G$ associated to the Shimura variety. Let $\operatorname{Spec}(k) \subset \mathcal{U}$ be a closed point that has characteristic different from $l$, then we have an isomorphism

$$
\left(R j_{*}\left({ }_{l} \mathcal{F} V\right)\right)_{k} \cong R j_{k *}\left({ }_{l} \mathcal{F} V \mid \mathfrak{x}_{k}\right)
$$

induced by the base change map.

Proof. We know that ${ }_{l} \mathcal{F} V$ is up to a Tate twist a summand of $R f_{*} \overline{\mathbb{Q}_{l}}$, where $f: \mathcal{A}^{\times n} \rightarrow \mathfrak{X}$ is the structure map of the $n$th fibre product of the universal abelian scheme $\mathcal{A} \rightarrow \mathfrak{X}$; that is, $\mathcal{A}^{\times n}:=\mathcal{A} \times \mathfrak{X} \cdots \times \times_{\mathfrak{X}} \mathcal{A}$, for some integer $n$. By [19] (Proposition 4.1), we have that $\mathcal{A}^{\times n}$ is $\mathbb{Z}_{(l)}^{\times}$-isogenous to another abelian scheme $Y$ over $\mathfrak{X}$ such that $Y$ extends to a proper scheme $\bar{Y}$ over $\mathfrak{X}_{\Sigma}^{\text {tor }}$ for some choice of smooth projective toroidal compactification $\mathfrak{X}_{\Sigma}^{\text {tor }} / \mathcal{U}$ with $\bar{Y} \backslash Y$ union of normal crossing divisors over $\mathcal{U}$. Because $\mathbb{Z}_{(l)}^{\times}{ }^{-}$ isogeny does not change Tate modules, we see that ${ }_{l} \mathcal{F} V$ is (up to a Tate twist) a summand of $R \pi_{*} \frac{\mathbb{Q}_{l}}{\text {, }}$, where $\pi: Y \rightarrow \mathfrak{X}$ the structure map. Thus, it suffices to show

$$
\left(R j_{*} R \pi_{*} \overline{\mathbb{Q}_{l}}\right)_{k} \cong R j_{k *}\left(\left.R \pi_{*} \overline{\mathbb{Q}_{l}}\right|_{\mathfrak{x}_{k}}\right) .
$$

Let $\bar{\pi}: \bar{Y} \rightarrow \mathfrak{X}_{\Sigma}^{\text {tor }}$ be the extension of $\pi, J: \mathfrak{X} \rightarrow \mathfrak{X}_{\Sigma}^{\text {tor }}, J_{Y}: Y \rightarrow \bar{Y}$ the inclusion, which form a Cartesian diagram

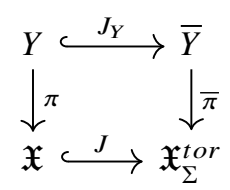


Let $\phi: \mathfrak{X}_{\Sigma}^{\text {tor }} \rightarrow \mathfrak{X}^{\text {min }}$ be the natural proper projection map. We denote by $\pi_{k}$ the base change of $\pi$ to $k$, and similarly for the other maps. We know that $j=\phi \circ J$, so

$$
\begin{gathered}
\left(R j_{*} R \pi_{*} \overline{\mathbb{Q}_{l}}\right)_{k}=\left(R \phi_{*} R J_{*} R \pi_{*} \overline{\mathbb{Q}_{l}}\right)_{k}=R \phi_{k *}\left(R J_{*} R \pi_{*} \overline{\mathbb{Q}_{l}}\right)_{k} \\
=R \phi_{k *}\left(R \bar{\pi}_{*} R J_{Y *} \overline{\mathbb{Q}_{l}}\right)_{k}=R \phi_{k *} R \bar{\pi}_{k *}\left(R J_{Y *} \overline{\mathbb{Q}_{l}}\right)_{k}
\end{gathered}
$$

by proper base change. Moreover,

$$
\left(R J_{Y *} \overline{\mathbb{Q}_{l}}\right)_{k}=R J_{Y k *} \overline{\mathbb{Q}_{l}}
$$

by Paragraph 5.1 .3 of section 7.5 (the appendix by Illusie) of SGA 4.5 ([6]), where we use that $\bar{Y} \backslash Y$ are the union of normal crossing divisors over $\mathcal{U}$. This gives

$$
\left(R j_{*} R \pi_{*} \overline{\mathbb{Q}_{l}}\right)_{k}=R \phi_{k *} R \bar{\pi}_{k *} R J_{Y k *} \overline{\mathbb{Q}_{l}}=R \phi_{k *} R J_{k *} R \pi_{k *} \overline{\mathbb{Q}_{l}}=R j_{k *}\left(\left.R \pi_{*} \overline{\mathbb{Q}_{l}}\right|_{\mathfrak{x}_{k}}\right)
$$

by proper base change again, proving the claim.

Lastly, we record the functoriality of the spectral sequence $E_{1}^{p, q}$.

Proposition 2.18. Let $\bar{X}$ and $\bar{Y}$ be varieties defined over a field $k$ that is either finitely generated over its prime field or the complex number, as in the previous section. Let $X \subset \bar{X}$ and $Y \subset \bar{Y}$ be nonempty open subvarieties and $\bar{f}: \bar{X} \rightarrow \bar{Y}$ a finite morphism that restricts to a morphism $f: X \rightarrow Y$ making the following diagram Cartesian:

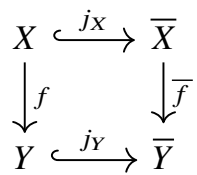

Let $\mathcal{F} \in D_{m}^{b}(X)$ and $\mathcal{G} \in D_{m}^{b}(Y)$ together with a morphism $h: \mathcal{G} \rightarrow R f_{*} \mathcal{F}$, then $h$ induces a morphism

$$
{ }_{Y} E_{1}^{p, q} \longrightarrow{ }_{X} E_{1}^{p, q}
$$

between the weight spectral sequences

$$
\begin{aligned}
& { }_{X} E_{1}^{p, q}=\mathbb{H}^{p+q}\left(\bar{X}, w_{\geq-p} w_{\leq-p} R j_{X *} \mathcal{F}\right) \Rightarrow \mathbb{H}^{p+q}(X, \mathcal{F}) \\
& { }_{Y} E_{1}^{p, q}=\mathbb{H}^{p+q}\left(\bar{Y}, w_{\geq-p} w_{\leq-p} R j_{Y *} \mathcal{G}\right) \Rightarrow \mathbb{H}^{p+q}(Y, \mathcal{G}) .
\end{aligned}
$$

In particular, the morphism $R^{p+q} \Gamma(Y,-)(h): \mathbb{H}^{p+q}(Y, \mathcal{G}) \rightarrow \mathbb{H}^{p+q}(X, \mathcal{F})$ respects filtrations induced by the spectral sequences.

Proof. Observe that $h$ induces a morphism

$$
R j_{Y *} \mathcal{G} \stackrel{R j_{Y *} h}{\longrightarrow} R j_{Y *} R f_{*} \mathcal{F}=R \bar{f}_{*} R j_{X *} \mathcal{F}
$$

and applying $R \Gamma(\bar{Y},-)$ to it recovers the usual morphism induced by $h$,

$$
R \Gamma(Y,-)(h): R \Gamma(Y, \mathcal{G}) \longrightarrow R \Gamma(X, \mathcal{F}),
$$

which is the sought-after morphism on $E_{\infty}$. Applying the functor $w_{\leq a}$ to (9) gives us

$$
w_{\leq a} R j_{Y *} \mathcal{G} \longrightarrow w_{\leq a} R \bar{f}_{*} R j_{X *} \mathcal{F}=R \bar{f}_{*} w_{\leq a} R j_{X *} \mathcal{F},
$$


where in the last equality we use that $\bar{f}$ is finite; hence $\bar{f}_{*}=\bar{f}_{\text {! }}$ preserves both ${ }^{w} D^{\leq a}$ and ${ }^{w} D^{\geq a}$ by proposition 2.7 ( $d=0$ as $f$ is finite). Now (10) shows that the morphism (9) preserves the filtration induced by the weight truncation $w_{\leq a}$, hence defining a morphism between spectral sequences as desired.

\section{PEL moduli problems}

\subsection{Kottwitz's PEL moduli problems}

We begin by recalling the definition of PEL moduli problems given by Kottwitz in [17]. We follow the notation of Lan ([18]).

Let $B$ be a finite-dimensional simple algebra over $\mathbb{Q}$ with a positive involution $*$ and $\mathcal{O}$ a $\mathbb{Z}$-order in $B$ that is invariant under $*$ and maximal at $p$, where $p$ is a rational prime that is unramified in $\mathrm{B}$; that is, $B_{\mathbb{Q}_{p}} \cong M_{n}(K)$ for some finite unramified extension $K$ of $\mathbb{Q}_{p}$. Let $L$ be an $\mathcal{O}$-lattice in a finitedimensional $B$-module $V$ and $\langle\cdot, \cdot\rangle: L \times L \rightarrow \mathbb{Z}(1)$ an alternating nondegenerate bilinear form on $L$ that satisfies $\langle\alpha x, y\rangle=\left\langle x, \alpha^{*} y\right\rangle$ for $\alpha \in \mathcal{O}$ and $x, y \in L$. We also assume that when localised at $p, L$ is self-dual with respect to $\langle\cdot, \cdot\rangle$. Here we denote $\mathbb{Z}(1):=\operatorname{Ker}\left(\exp : \mathbb{C} \rightarrow \mathbb{C}^{*}\right)$. A choice of $\sqrt{-1}$ gives an identification of it with $\mathbb{Z}$, but we do not fix such an identification.

We assume that there is an $\mathbb{R}$-algebra homormorphism $h: \mathbb{C} \rightarrow \operatorname{End}_{\mathcal{O}_{\mathbb{R}}}\left(L_{\mathbb{R}}\right)$ such that $\langle h(z) x, y\rangle_{\mathbb{R}}=$ $\langle x, h(\bar{z}) y\rangle_{\mathbb{R}}$ and $\langle\cdot, h(\sqrt{-1}) \cdot\rangle_{\mathbb{R}}$ is symmetric and positive definite. If we fix an identification $\mathbb{Z}(1) \cong \mathbb{Z}$ so that $\langle\cdot, \cdot\rangle_{\mathbb{R}}$ takes values in $\mathbb{R}$. Let

$$
G^{*}(R):=\left\{(g, r) \in G L_{\mathcal{O}_{R}}\left(L_{R}\right) \times \mathbf{G}_{m}(R):\langle g x, g y\rangle=r\langle x, y\rangle, \forall x, y \in L_{R}\right\}
$$

for a $\mathbb{Z}$-algebra $R$. This defines an algebraic group $G^{*}$ over $\mathbb{Z}$. We assume that the derived group has type $\mathbf{A}$ or $\mathbf{C}$ in the classification.

The morphism $h$ defines a decomposition $L \otimes \mathbb{C}=V_{0} \oplus V_{0}^{c}$, where $h(z)$ acts as $1 \otimes z$ on $V_{0}$ and $1 \otimes \bar{z}$ on $V_{0}^{c}$. We know that $V_{0}$ is an $\mathcal{O} \otimes \mathbb{C}$-module because $h(z)$ commutes with $\mathcal{O}_{\mathbb{R}}$ by definition. The reflex field $F_{0}$ is defined to be the field of definition of $V_{0}$ as an $\mathcal{O} \otimes \mathbb{C}$-module; see [18] Definition 1.2.5.4 for more details.

Definition 3.1. Let $\mathcal{H}$ be an open compact subgroup of $G^{*}\left(\mathbb{A}^{p \infty}\right), M_{\mathcal{H}}^{r a t}$ is defined to be the category fibred in groupoids over the category of locally Noetherian schemes defined over $\mathcal{O}_{F_{0}} \otimes \mathbb{Z}_{(p)}$, whose fibre over $S$ consists of tuples

$$
\left(A, \lambda, i,[\hat{\alpha}]_{\mathcal{H}}\right),
$$

where $A$ is an abelian scheme over $\mathrm{S}$,

$$
\lambda: A \rightarrow A^{\vee}
$$

is a prime-to- $p$ quasi-polarisation of $A$ and

$$
i: \mathcal{O} \otimes \mathbb{Z}_{(p)} \rightarrow \operatorname{End}_{S}(A) \otimes \mathbb{Z}_{(p)}
$$

is a ring homomorphism such that

$$
i(b)^{\vee} \circ \lambda=\lambda \circ i\left(b^{*}\right)
$$

for every $b \in \mathcal{O} \otimes \mathbb{Z}_{(p)}$ and Lie $_{A / S}$ satisfies the determinant condition specified by $h$; see [18] Definition 1.3.4.1 for a precise formulation. Moreover, if we choose a geometric point $\bar{s}$ in each connected component of $S,[\hat{\alpha}]_{\mathcal{H}}$ is an assignment to each $\bar{s}$ a $\pi_{1}(S, \bar{s})$-invariant $\mathcal{H}$-orbit of $\mathcal{O} \otimes \mathbb{A}^{p \infty}$-equivariant isomorphisms

$$
\hat{\alpha}: L \otimes \mathbb{A}^{p \infty} \stackrel{\sim}{\rightarrow} V^{p} A_{\bar{s}}
$$


together with an isomorphism

$$
v(\hat{\alpha}): \mathbb{A}^{p \infty}(1) \stackrel{\sim}{\rightarrow} V^{p}\left(\mathbf{G}_{m, \bar{s}}\right)
$$

such that

$$
\langle\hat{\alpha}(x), \hat{\alpha}(y)\rangle_{\lambda}=v(\hat{\alpha}) \circ\langle x, y\rangle,
$$

where $x, y \in L \otimes \mathbb{A}^{p \infty},\langle\cdot, \cdot\rangle_{\lambda}$ is the Weil pairing associated to the polarisation $\lambda$, and $V^{p}$ is the prime to $p$ rational Tate module of either $A$ or $\mathbf{G}_{m}$.

The isomorphisms in the groupoid are defined to be $\left(A, \lambda, i,[\hat{\alpha}]_{\mathcal{H}}\right) \sim\left(A^{\prime}, \lambda^{\prime}, i^{\prime},\left[\hat{\alpha}^{\prime}\right]_{\mathcal{H}}\right)$ if and only if there is a prime to $p$ quasi-isogeny $f: A \rightarrow A^{\prime}$ such that over each connected component of $S$,

$$
\lambda=r f^{\vee} \circ \lambda^{\prime} \circ f
$$

for some $r \in \mathbb{Z}_{(p),>0}^{\times}, f \circ i(b)=i^{\prime}(b) \circ f$ for all $b \in \mathcal{O} \otimes \mathbb{Z}_{(p)}$. Moreover, we require that for each geometric point $\bar{s}$ of $S, \hat{\alpha}^{\prime-1} \circ V^{p}(f) \circ \hat{\alpha} \in \mathcal{H}$, and $v\left(\hat{\alpha}^{\prime}\right)^{-1} \circ v(\hat{\alpha}) \in v(\mathcal{H}) r \subset \mathbb{A}^{p \infty, \times}$ for the $r$ specified by $\lambda=r f^{\vee} \circ \lambda^{\prime} \circ f$ at $\bar{s}$.

Remark 3.2. Note that the moduli problem depends only on the $B$-module $V$, and different choices of $L$ will only affect the choices of maximal compact subgroup of $G^{*}\left(\mathbb{A}^{p \infty}\right)$. This will be helpful when we consider moduli problems defined by isomorphism classes where the choice of $L$ is important. We can compare different moduli problems defined by different choices of $L$ by identifying the moduli problems with the above one using isogeny classes.

Remark 3.3. We work with locally Noetherian test schemes rather than arbitrary schemes because etale fundamental groups do not behave well for general schemes. General (affine) schemes can be written as inverse limits of locally Noetherian schemes, and we can extend the moduli functor to the general case by taking limits.

Remark 3.4. $v(\hat{\alpha})$ is a rigidification of Kottwitz's definition of PEL moduli problems, where he allows the ambiguity that the Weil pairing is equal to the fixed pairing $\langle\cdot, \cdot\rangle$ up to a similitude factor. If $L \neq 0$, $v(\hat{\alpha})$ is uniquely determined by $\hat{\alpha}$; hence the two definitions are equivalent. If $L=0, v(\hat{\alpha})$ is the only nontrivial data. We include the $L=0$ case because it will appear in the boundary of the minimal compactification of PEL Shimura varieties.

It is not hard to see that the moduli space is represented by an algebraic stack that is smooth of finite type over $\mathcal{O}_{F_{0}} \otimes \mathbb{Z}_{(p)}$, and it is even represented by a finite-type smooth scheme over $\mathcal{O}_{F_{0}} \otimes \mathbb{Z}_{(p)}$ if $\mathcal{H}$ is small enough. We will use the same symbol $M_{\mathcal{H}}^{r a t}$ to denote the stack or scheme it represents.

The above definition uses isogeny classes of abelian varieties. We will next define another moduli problem using isomorphism classes. This is necessary for the toroidal compactifications because semiabelian varieties do not behave well under isogeny.

We will only define moduli problems for principal level structures; the general level structures can be defined by taking orbits of the principal ones, but we choose to ignore them for reasons to be explained later.

Definition 3.5. Let $n$ be a natural number prime to $p$ and define $M_{n}$ to be the category fibred in groupoids over the category of schemes over $\mathcal{O}_{F_{0}} \otimes \mathbb{Z}_{(p)}$, whose fibre over $S$ is the groupoids with object tuples

$$
\left(A, \lambda, i,\left(\alpha_{n}, v_{n}\right)\right),
$$

where $A$ is an abelian scheme over $S$,

$$
\lambda: A \rightarrow A^{\vee}
$$

is a prime-to- $p$ polarisation and

$$
i: \mathcal{O} \rightarrow \operatorname{End}_{S}(A)
$$


a ring homomorphism such that $i(b)^{\vee} \circ \lambda=\lambda \circ i\left(b^{*}\right)$ for every $b \in \mathcal{O}$. We require that $\mathrm{Lie}_{A / S}$ satisfies the determinant condition given by $h$. The principal level-n structure is an $\mathcal{O}$-equivariant isomorphism

$$
\alpha_{n}:(L / n L)_{S} \stackrel{\sim}{\rightarrow} A[n]
$$

together with an isomorphism

$$
v_{n}:(\mathbb{Z} / n \mathbb{Z}(1))_{S} \stackrel{\sim}{\rightarrow} \mu_{n, S}
$$

of group schemes over S such that $\left\langle\alpha_{n}(x), \alpha_{n}(y)\right\rangle_{\lambda}=v_{n} \circ\langle x, y\rangle$ for $x, y \in(L / n L)_{S}$. The $\left(\alpha_{n}, v_{n}\right)$ has to satisfy a sympletic-liftablity condition that roughly says that it can be lifted to a level-m structure for arbitary $m$ that is prime to $\mathrm{p}$ and divisible by $n$; see [18] Definition 1.3.6.2 for precise definitions.

The isomorphisms in the groupoid are defined to be

$$
\left(A, \lambda, i,\left(\alpha_{n}, v_{n}\right)\right) \sim\left(A^{\prime}, \lambda^{\prime}, i^{\prime},\left(\alpha_{n}^{\prime}, v_{n}^{\prime}\right)\right)
$$

if and only if there is an isomorphism $f: A \rightarrow A^{\prime}$ such that

$$
\lambda=f^{\vee} \circ \lambda^{\prime} \circ f
$$

$f \circ i(b)=i^{\prime}(b) \circ f$ for all $b \in \mathcal{O}$ and $\alpha_{n}^{\prime}=f \circ \alpha_{n}, v_{n}=v_{n}^{\prime}$.

Let

$$
\mathcal{U}(n):=\operatorname{Ker}\left(G^{*}\left(\hat{\mathbb{Z}}^{p}\right) \rightarrow G^{*}(\mathbb{Z} / n \mathbb{Z})\right),
$$

then we can show that $M_{n} \cong M_{\mathcal{U}(n)}^{\text {rat }}$, the map being the obvious one sending PEL abelian varieties to their isogeny classes. The inverse map is to choose an abelian variety in each isogeny class, determined by the choice of the $\mathcal{O}$-lattice $L$ inside $B$-module $V$; see [18] Section 1.4 .3 for a careful proof of the isomorphism. One subtle point is that $M_{n}$ and $M_{U(n)}^{\text {rat }}$ are defined over different categories of test schemes. We can show that $M_{n}$ is determined by its value on locally Noetherian schemes by writing any (affine) scheme as an inverse limit of locally Noetherian ones and note that the moduli functor $M_{n}$, being fintely presented, commutes with inverse limits. See also Remark 3.3.

\subsection{Similitude PEL moduli problems}

Let $F^{c}$ be the center of $B$, which is a number field by simplicity of $B$. Let $F:=F^{c, *=1}$, and we assume that $\mathcal{O}_{F} \subset \mathcal{O}$. We define a group scheme $H$ over $\mathcal{O}_{F}$ by

$$
H(R):=\left\{(g, r) \in G L_{\mathcal{O}_{\otimes_{\mathcal{O}}} R}\left(L \otimes_{\mathcal{O}_{F}} R\right) \times \mathbf{G}_{m}(R):\langle g x, g y\rangle=r\langle x, y\rangle, \forall x, y \in L \otimes_{\mathcal{O}_{F}} R\right\}
$$

for an $\mathcal{O}_{F}$-algebra $R$. Let

$$
G:=\operatorname{Res}_{\mathcal{O}_{F} / \mathbb{Z}} H
$$

and we have the similitude map $v: G \rightarrow \operatorname{Res}_{\mathcal{O}_{F} / \mathbb{Z}} \mathbf{G}_{m}$; then

$$
G^{*}=v^{-1}\left(G_{m, \mathbb{Z}}\right) \subset G .
$$

Note that $h: \mathbb{C} \rightarrow \operatorname{End}_{\mathcal{O}_{\mathbb{R}}}\left(L_{\mathbb{R}}\right)$ defines a Deligne cocharacter $\operatorname{Res}_{\mathbb{C} / \mathbb{R}} \mathbf{G}_{m} \rightarrow G^{*}$ and hence also $\operatorname{Res}_{\mathbb{C} / \mathbb{R}} \mathbf{G}_{m} \rightarrow G$. Their conjugacy classes define Shimura varieties associated to $G$ and $G^{*}$, which we will denote by $S h_{K}(G, h)$ and $S h_{\mathcal{H}}\left(G^{*}, h\right)$ for compact open subgroups $K \subset G\left(\hat{\mathbb{Z}}^{p}\right)$ and $\mathcal{H} \subset$ $G^{*}\left(\hat{\mathbb{Z}}^{p}\right)$. These are abbreviated notions for $S h_{K G\left(\mathbb{Z}_{p}\right)}(G, h)$ and $S h_{\mathcal{H} G^{*}\left(\mathbb{Z}_{p}\right)}\left(G^{*}, h\right)$, which might be more standard. 
We have made the assumption that our PEL datum has type $\mathbf{A}$ or $\mathbf{C}$; then $M_{\mathcal{H}}^{r a t}$ is an integral model of the Shimura variety $S h_{\mathcal{H}}\left(G^{*}, h\right)$ in case $(\mathbf{A}$, even $)$ or $\mathbf{C}$. In the case $(\mathbf{A}$, odd $), M_{\mathcal{H}}^{\text {rat }}$ is a disjoint union of integral models of the Shimura variety $S h_{\mathcal{H}}\left(G^{*}, h\right)$, due to the failure of Hasse principle.

We will be working with the Shimura variety associated to $G$ instead of $G^{*}$, and one advantage of $G$ is that it always satisfies the Hasse principle. For our purpose, the more important reasons are that Shimura varieties associated to $G$ are plectic, whereas Shimura varieties of $G^{*}$ are only plectic in positive dimension. More precisely, the difference between the two Shimura varieties is that they have different sets of connected components, and we have $\pi_{0}\left(S h_{K}(G, h)\right)$ is plectic while $\pi_{0}\left(S h_{\mathcal{H}}\left(G^{*}, h\right)\right)$ is not; that is, $\pi_{0}\left(S h_{K}(G, h)\right)$ is the 0-dimensional Shimura variety associated to $\operatorname{Res}_{F / \mathbb{Q}} \mathbf{G}_{m}$ (plectic), whereas $\pi_{0}\left(S h_{\mathcal{H}}\left(G^{*}, h\right)\right)$ has group $\mathbf{G}_{m}$ (not plectic). The plectic nature of $S h_{K}(G, h)$ will give rise to the so-called partial Frobenius, which will play an important role in our study.

On the other hand, the price to pay when changing to $G$ is that we do not have a good fine moduli problem represented by $S h_{K}(G, h)$. Instead, $S h_{K}(G, h)$ will only be a coarse moduli space. We now give the moduli problem of $S h_{K}(G, h)$. We will follow Nekováŕr's approach as in [25].

Definition 3.6. Fix $\alpha \in\left(F \otimes \mathbb{A}^{p \infty}\right)^{\times}$and $K \subset G\left(\hat{\mathbb{Z}}^{p}\right)$ open compact subgroup and let us define $\mathscr{M}_{\alpha, K}$ to be the category fibred in groupoids over the category of locally Noetherian schemes over $\mathcal{O}_{F_{0}} \otimes \mathbb{Z}_{(p)}$, whose objects over $S$ are quadruples

$$
(A, \lambda, i, \overline{(\eta, u)}) \text {, }
$$

where $A$ is an abelian scheme over $\mathrm{S}$,

$$
\lambda: A \rightarrow A^{\vee}
$$

is a prime-to- $p$ quasi-polarisation of $A$ and

$$
i: \mathcal{O} \rightarrow \operatorname{End}_{S}(A)
$$

is a ring homomorphism such that $i(b)^{\vee} \circ \lambda=\lambda \circ i\left(b^{*}\right)$ for every $b \in \mathcal{O}$, and Lie $_{A / S}$ satisfies the determinant condition specified by $h$; see [18] Definition 1.3.4.1 for a precise formulation. Moreover, if we choose a geometric point $\bar{s}$ in each connected component of $S$, the level structure $\overline{(\eta, u)}$ is an assignment to each $\bar{s}$ a $\pi_{1}(S, \bar{s})$-invariant $K$-orbit of $\mathcal{O} \otimes \mathbb{A}^{p \infty}$-equivariant isomorphisms

$$
\eta: L \otimes \mathbb{A}^{p \infty} \stackrel{\sim}{\rightarrow} V^{p} A_{\bar{s}}
$$

together with an $\mathcal{O}_{F} \otimes \hat{\mathbb{Z}}^{p}$-equivariant isomorphism

$$
u: \mathfrak{D}_{F}^{-1} \otimes \hat{\mathbb{Z}}^{p}(1) \stackrel{\sim}{\rightarrow} T^{p}\left(\mathfrak{D}_{F}^{-1} \otimes_{\mathbb{Z}} \mathbf{G}_{m, \bar{s}}\right)
$$

such that

$$
\langle\eta(x), \eta(y)\rangle_{\lambda}=\operatorname{Tr}_{\mathcal{O}_{F} / \mathbb{Z}}\left(u \circ\left(\alpha\langle x, y\rangle_{F}\right)\right),
$$

where $x, y \in L \otimes \mathbb{A}^{p \infty}, \mathfrak{D}_{F}^{-1}$ is the inverse different of $F$ and $u$ extends naturally from $\mathfrak{D}_{F}^{-1} \otimes \mathbb{A}^{p \infty}(1)$ to the rational Tate module. Here $\mathfrak{b}_{F}^{-1} \otimes_{\mathbb{Z}} \mathbf{G}_{m, \bar{s}}$ is defined in the category of fppf sheaf of abelian groups, which can be easily seen to be representable. The $\mathcal{O}_{F}$-action on the first factor equips $\mathfrak{D}_{F}^{-1} \otimes_{\mathbb{Z}} \mathbf{G}_{m, \bar{s}}$ with an action of $\mathcal{O}_{F}$ and hence defines a $\mathcal{O}_{F} \otimes \hat{\mathbb{Z}}^{p}$-module structure on the Tate module $T^{p}\left(\mathfrak{D}_{F}^{-1} \otimes_{\mathbb{Z}} \mathbf{G}_{m, \bar{s}}\right)$. The $\operatorname{Tr}_{\mathcal{O}_{F} / \mathbb{Z}}: \grave{D}_{F}^{-1} \otimes_{\mathbb{Z}} \mathbf{G}_{m, \bar{s}} \rightarrow \mathbb{Z} \otimes_{\mathbb{Z}} \mathbf{G}_{m, \bar{s}}$ is the trace map on the first factor. Moreover,

$$
\langle\cdot, \cdot\rangle_{F}: L \times L \rightarrow \mathfrak{D}_{F}^{-1} \otimes \mathbb{Z}(1)
$$

is the unique $\mathcal{O}_{F}$-linear pairing such that

$$
\operatorname{Tr}_{\mathcal{O}_{F} / \mathbb{Z}} \circ\langle\cdot, \cdot\rangle_{F}=\langle\cdot, \cdot\rangle
$$


The action of $K$ on $(\eta, u)$ is given by $(\eta, u) g=(\eta \circ g, u \circ v(g))$ for $g \in K$, where $v(g) \in\left(\mathcal{O}_{F} \otimes \hat{\mathbb{Z}}^{p}\right)^{\times}$ acts on $\mathfrak{D}_{F}^{-1} \otimes \hat{\mathbb{Z}}^{p}(1)$ in the obvious way.

The isomorphisms in the groupoid are defined to be

$$
(A, \lambda, i, \overline{(\eta, u)}) \sim\left(A^{\prime}, \lambda^{\prime}, i^{\prime}, \overline{\left(\eta^{\prime}, u^{\prime}\right)}\right)
$$

if and only if there is a prime to $p$ quasi-isogeny $f: A \rightarrow A^{\prime}$ such that over each connected component of $S$,

$$
\lambda=f^{\vee} \circ \lambda^{\prime} \circ f
$$

$f \circ i(b)=i^{\prime}(b) \circ f$ for all $b \in \mathcal{O} \otimes \mathbb{Z}_{(p)}$ and $\overline{\left(\eta^{\prime}, u^{\prime}\right)}=\overline{(f \circ \eta, u)}$.

Remark 3.7. The above moduli problem is in some sense in between the isogeny classes and the isomorphism classes moduli problems, in that it uses isogeny as morphisms in the groupoid and rational Tate modules for level structures, while also encoding integral structures in endomorphism structures and $u$. This has the effect that the morphism $f$ in the groupoid is required to strictly preserve the polarisation $\lambda=f^{\vee} \circ \lambda^{\prime} \circ f$ without the factor $r$ in Definition 3.1.

It is not hard to see that $\mathscr{M}_{\alpha, K}$ is representable by a smooth quasi-projective scheme $M_{\alpha, K}$ over $\mathcal{O}_{F_{0}} \otimes \mathbb{Z}_{(p)}$.

There is an action of totally positive prime to $p$ units $\left(\mathcal{O}_{F}\right)_{+}^{\times}$on $\mathscr{M}_{\alpha, K}$ given by the formula

$$
\epsilon \cdot(A, \lambda, i, \overline{(\eta, u)})=(A, i(\epsilon) \lambda, i, \overline{(\eta, \epsilon u)})
$$

that factors through the finite quotient group $\Delta:=\left(\mathcal{O}_{F}\right)_{+}^{\times} / N m_{F^{c} / F}\left(\left(\mathcal{O}_{F^{c}}\right)^{\times} \cap K\right)$. The quotient $M_{\alpha, K} / \Delta$ always exists. Let $\Omega=\{\alpha\} \subset\left(F \otimes \mathbb{A}^{(p \infty)}\right)^{\times}$be a set of representatives of the double cosets

$$
\left(F \otimes \mathbb{A}^{(p \infty)}\right)^{\times}=\coprod_{\alpha \in \Omega}\left(\mathcal{O}_{F} \otimes \mathbb{Z}_{(p)}\right)_{+}^{\times} \alpha\left(\mathcal{O}_{F} \otimes \hat{\mathbb{Z}}^{p}\right)^{\times},
$$

then we have

$$
S h_{K}(G, h)=\coprod_{\alpha \in \Omega} M_{\alpha, K} / \Delta=M_{K} / \Delta,
$$

where $M_{K}:=\coprod_{\alpha \in \Omega} M_{\alpha, K}$. This means that $M_{K} / \Delta$ has the same complex points as $S h_{K}(G, h)$, hence defining an integral model of $S h_{K}(G, h)$. This is a consequence of the fact that $G$ satisfies the Hasse principle; see [14] Section 7.1.5.

Remark 3.8. We can show that $M_{K} / \Delta$ is the coarse moduli space of the functor sending $S$ to quadruples

$$
(A, \lambda, i, \overline{(\eta, u)})
$$

where $A$ is an abelian scheme over $\mathrm{S}$,

$$
\lambda: A \rightarrow A^{\vee}
$$

is a prime to $p$ quasi-polarisation of $A$ and

$$
i: \mathcal{O} \otimes \mathbb{Z}_{(p)} \rightarrow \operatorname{End}_{S}(A) \otimes \mathbb{Z}_{(p)}
$$

is a ring homomorphism such that $i(b)^{\vee} \circ \lambda=\lambda \circ i\left(b^{*}\right)$ for every $b \in \mathcal{O} \otimes \mathbb{Z}_{(p)}$, and $L i e_{A / S}$ satisfies the determinant condition specified by $h$; see [18] Definition 1.3.4.1 for a precise formulation. Moreover, if we choose a geometric point $\bar{s}$ in each connected component of $S$, the level structure $\overline{(\eta, u)}$ is an assignment to each $\bar{s}$ a $\pi_{1}(S, \bar{s})$-invariant $K$-orbit of $\mathcal{O} \otimes \mathbb{A}^{p \infty}$-equivariant isomorphisms

$$
\eta: L \otimes \mathbb{A}^{p \infty} \stackrel{\sim}{\rightarrow} V^{p} A_{\bar{s}}
$$


together with an $F \otimes \mathbb{A}^{p \infty}$-equivariant isomorphism

$$
u: F \otimes \mathbb{A}^{p \infty}(1) \stackrel{\sim}{\rightarrow} V^{p}\left(\mathfrak{D}_{F}^{-1} \otimes_{\mathbb{Z}} \mathbf{G}_{m, \bar{s}}\right)
$$

such that

$$
\langle\eta(x), \eta(y)\rangle_{\lambda}=\operatorname{Tr}_{\mathcal{O}_{F} / \mathbb{Z}}\left(u \circ\langle x, y\rangle_{F}\right),
$$

where $x, y \in L \otimes \mathbb{A}^{p \infty}$.

The isomorphisms in the groupoid are defined to be

$$
(A, \lambda, i, \overline{(\eta, u)}) \sim\left(A^{\prime}, \lambda^{\prime}, i^{\prime}, \overline{\left(\eta^{\prime}, u^{\prime}\right)}\right)
$$

if and only if there is a prime to $p$ quasi-isogeny $f: A \rightarrow A^{\prime}$ such that over each connected component of $S$,

$$
\lambda \circ i(a)=f^{\vee} \circ \lambda^{\prime} \circ f
$$

for some $a \in\left(\mathcal{O}_{F} \otimes \mathbb{Z}_{(p)}\right)_{+}^{\times}, f \circ i(b)=i^{\prime}(b) \circ f$ for all $b \in \mathcal{O} \otimes \mathbb{Z}_{(p)}$ and $\overline{\left(\eta^{\prime}, u^{\prime}\right)}=\overline{(f \circ \eta, u \circ a)}$.

Note that this functor kills all of the integral structures in Definition 3; see Remark 4. Moreover, it enlarges the domain of ambiguity factor $r$ in Definition 1 from $\left(\mathbb{Z}_{(p)}\right)_{+}^{\times}$to $\left(\mathcal{O}_{F} \otimes \mathbb{Z}_{(p)}\right)_{+}^{\times}$.

See [14] Section 7.1.3 for more details on this moduli problem.

We will work with integral toroidal and minimal compactifications of $S h_{K}(G, h)$. However, this has only been constructed by Lan for the PEL moduli problems in Definitions 3.1 and 3.5. Fortunately, $S h_{K}(G, h)$ is not very different from $S h_{\mathcal{H}}\left(G^{*}, h\right)$. The precise relation is that for each $\mathcal{H} \subset G^{*}\left(\hat{\mathbb{Z}}^{p}\right)$, there exists an open compact subgroup $K \subset G\left(\hat{\mathbb{Z}}^{p}\right)$ containing $\mathcal{H}$ such that the natural map $S h_{\mathcal{H}}\left(G^{*}, h\right) \rightarrow$ $S h_{K}(G, h)$ induced by $G^{*} \subset G$ is an open immersion containing the identity component of $S h_{K}(G, h)$, and the Hecke translates of $S h_{\mathcal{H}}\left(G^{*}, h\right)$ cover $S h_{K}(G, h)$; see, for example, [7] Proposition 1.15. We need a more explicit description of $S h_{K}(G, h)$ in terms of $S h_{\mathcal{H}}\left(G^{*}, h\right)$, so we focus on principal level structures from now on.

Suppose that $n$ is prime to $p$ and let

$$
K(n):=\operatorname{Ker}\left(G\left(\hat{\mathbb{Z}}^{p}\right) \rightarrow G(\mathbb{Z} / n \mathbb{Z})\right) .
$$

Observe that

$$
v(K(n))=\operatorname{Ker}\left(\left(\mathcal{O}_{F} \otimes_{\mathbb{Z}} \mathbf{G}_{m}\right)\left(\hat{\mathbb{Z}}^{p}\right) \rightarrow\left(\mathcal{O}_{F} \otimes_{\mathbb{Z}} \mathbf{G}_{m}\right)(\mathbb{Z} / n \mathbb{Z})\right) .
$$

Recall that

$$
\mathcal{U}(n):=\operatorname{Ker}\left(G^{*}\left(\hat{\mathbb{Z}}^{p}\right) \rightarrow G^{*}(\mathbb{Z} / n \mathbb{Z})\right)
$$

and

$$
v(\mathcal{U}(n))=\operatorname{Ker}\left(\mathbf{G}_{m}\left(\hat{\mathbb{Z}}^{p}\right) \rightarrow \mathbf{G}_{m}(\mathbb{Z} / n \mathbb{Z})\right)
$$

Choose a set $\Lambda$ of representatives of the double quotient

$$
\left(\mathcal{O}_{F} \otimes \hat{\mathbb{Z}}^{p}\right)^{\times}=\coprod_{\delta \in \Lambda}\left(\mathcal{O}_{F}\right)_{+}^{\times} \delta\left(v(K(n)) \hat{\mathbb{Z}}^{p, \times}\right),
$$

then together with the representatives $\Omega$,

$$
\left(F \otimes \mathbb{A}^{(p \infty)}\right)^{\times}=\coprod_{\alpha \in \Omega}\left(\mathcal{O}_{F} \otimes \mathbb{Z}_{(p)}\right)_{+}^{\times} \alpha\left(\mathcal{O}_{F} \otimes \hat{\mathbb{Z}}^{p}\right)^{\times},
$$


we have the decomposition

$$
\left(F \otimes \mathbb{A}^{(p \infty)}\right)^{\times}=\coprod_{\substack{\alpha \in \Omega \\ \delta \in \Lambda}}\left(\mathcal{O}_{F} \otimes \mathbb{Z}_{(p)}\right)_{+}^{\times} \alpha \delta\left(v(K(n)) \hat{\mathbb{Z}}^{p, \times}\right)
$$

Let us change the notation $M_{n}$ in Definition 3.5 into $M_{n}(L,\langle\cdot, \cdot\rangle)$ to emphasise the dependence of $M_{n}$ on $L$ and $\langle\cdot, \cdot\rangle$. Then there is a natural embedding

$$
M_{n}\left(L, \operatorname{Tr}_{\mathcal{O}_{F} / \mathbb{Z}} \circ\left(\alpha \delta\langle\cdot, \cdot\rangle_{F}\right)\right) \hookrightarrow M_{\alpha, K(n)} / \Delta
$$

sending $\left(A, \lambda, i,\left(\alpha_{n}, v_{n}\right)\right)$ to $(A, \lambda, i, \overline{(\eta, u)})$, where $\eta: L \otimes \mathbb{A}^{p \infty} \stackrel{\sim}{\rightarrow} V^{p} A_{\bar{s}}$ is defined by a lifting of $\alpha_{n}$ to

$$
\hat{\alpha}: L \otimes \hat{\mathbb{Z}}^{p} \stackrel{\sim}{\rightarrow} T^{p} A_{\bar{s}}
$$

whose existence is a condition on the level structure in Definition 3.5, and then inverting all prime to $\mathrm{p}$ integers. $u: \mathfrak{D}_{F}^{-1} \otimes \hat{\mathbb{Z}}^{p}(1) \stackrel{\sim}{\rightarrow} T^{p}\left(\mathfrak{D}_{F}^{-1} \otimes_{\mathbb{Z}} \mathbf{G}_{m, \bar{s}}\right)$ is defined by

$$
\langle\eta(x), \eta(y)\rangle_{\lambda}=\operatorname{Tr}_{\mathcal{O}_{F} / \mathbb{Z}}\left(u \circ\left(\alpha \delta\langle x, y\rangle_{F}\right)\right)
$$

for $x, y \in L \otimes \mathbb{A}^{p \infty}$. Here we abuse notation by denoting both $u$ and $u \otimes i d_{\mathbb{Z}_{(p)}}$ by $u$. The $K(n)$-class of $(\eta, u)$ does not depend on the choice of the lifting $\hat{\alpha}$.

Warning 3.9. $\left.\operatorname{Tr}_{\mathcal{O}_{F} / \mathbb{Z}} \circ\left(\alpha \delta\langle\cdot, \cdot\rangle_{F}\right)\right)$ is not defined on $L$ but rather on $L \otimes \hat{\mathbb{Z}}^{p}$ because $\alpha \delta \in \mathcal{O}_{F} \otimes \hat{\mathbb{Z}}^{p}$ if we choose $\alpha \in \mathcal{O}_{F} \otimes \hat{\mathbb{Z}}^{p}$, which we assume from now on. This does not affect the moduli problem because the moduli problem in Definition 3.5 only depends on $L \otimes \hat{\mathbb{Z}}^{p}, L \otimes \mathbb{R}$ and the corresponding pairing on them.

The appropriate notation for $M_{n}\left(L, \operatorname{Tr}_{\mathcal{O}_{F} / \mathbb{Z}} \circ\left(\alpha \delta\langle\cdot, \cdot\rangle_{F}\right)\right)$ would be $M_{n}\left(L,\langle\cdot, \cdot\rangle_{1}\right)$ for some pairing $\langle\cdot, \cdot\rangle_{1}$ on $L$ that is isomorphic to $\operatorname{Tr}_{\mathcal{O}_{F} / \mathbb{Z}} \circ\left(\alpha \delta\langle\cdot, \cdot\rangle_{F}\right)$ on $L \otimes \hat{\mathbb{Z}}^{p}$, perfect on $L \otimes \mathbb{Z}_{p}$ and compatible with $h$ on $L \otimes \mathbb{R}$. Such a pairing exists if the moduli problem is nonempty. See [18] Remark 1.4.3.14 for more explanations.

We use the wrong notation $M_{n}\left(L, \operatorname{Tr}_{\mathcal{O}_{F} / \mathbb{Z}} \circ\left(\alpha \delta\langle\cdot, \cdot\rangle_{F}\right)\right)$ for simplicity.

By definition of the moduli problem, the decomposition (14) gives

$$
M_{\alpha, K(n)} / \Delta=\coprod_{\delta \in \Lambda} M_{n}\left(L, \operatorname{Tr}_{\mathcal{O}_{F} / \mathbb{Z}} \circ\left(\alpha \delta\langle\cdot, \cdot\rangle_{F}\right)\right) .
$$

Then it follows from (16) and the definition of $M_{K(n)} / \Delta$ that

$$
M_{K(n)} / \Delta=\coprod_{\substack{\alpha \in \Omega \\ \delta \in \Lambda}} M_{n}\left(L, \operatorname{Tr}_{\mathcal{O}_{F} / \mathbb{Z}} \circ\left(\alpha \delta\langle\cdot, \cdot\rangle_{F}\right)\right)
$$

This will help us construct toroidal and minimal compactifications of $M_{K(n)} / \Delta$ from those constructed by Lan. We briefly recall Lan's results on minimal compactifications.

Theorem 3.10 (Lan [18]). There exists a compactification $M_{n}(L,\langle\cdot, \cdot\rangle)^{\text {min }}$ of $M_{n}(L,\langle\cdot, \cdot\rangle)$ together with a stratification by locally closed subschemes

$$
M_{n}(L,\langle\cdot, \cdot\rangle)^{\text {min }}=\coprod_{\left[\left(Z_{n}, \Phi_{n}, \delta_{n}\right)\right]} M_{n}\left(L^{Z_{n}},\langle\cdot, \cdot\rangle^{Z_{n}}\right)
$$


where

(1) $Z_{n}$ is a $\mathcal{O}$-invariant filtration on $L / n L$,

$$
0 \subset Z_{n,-2} \subset Z_{n,-1} \subset Z_{n, 0}=L / n L,
$$

that can be lifted to a $\mathcal{O}$-invariant filtration $Z$ on $L \otimes \hat{\mathbb{Z}}^{p}$,

$$
0 \subset Z_{-2} \subset Z_{-1} \subset Z_{0}=L \otimes \hat{\mathbb{Z}}^{p},
$$

such that $Z$ is the restriction of a split $\mathcal{O}$-invariant filtration $Z_{\mathbb{A}^{p}}$ on $L \otimes \mathbb{A}^{p}$ satisfying $Z_{\mathbb{A} p,-2}^{\perp}=Z_{\mathbb{A}} p,-1$ and $G r_{i} Z_{\mathbb{A} p} \cong L_{i} \otimes \mathbb{A}^{p}$ for some $\mathcal{O}$-lattice $L_{i}$. Let $L^{\mathbb{Z}_{n}}:=L_{-1}$ and $\langle\cdot, \cdot\rangle^{\mathbb{Z}_{n}}$ be a pairing on $L^{\mathbb{Z}_{n}}$ that induces $\langle\cdot, \cdot\rangle$ on $G r_{-1} Z_{\mathbb{A} p}$. There exists an $h^{Z_{n}}: \mathbb{C} \rightarrow \operatorname{End}_{\mathcal{O}_{\mathbb{R}}}\left(L_{\mathbb{R}}^{Z_{n}}\right)$ that makes $\left(L^{Z_{n}},\langle\cdot, \cdot\rangle^{Z_{n}}, h^{Z_{n}}\right)$ a PEL data defining the moduli problem $M_{n}\left(L^{Z_{n}},\langle\cdot, \cdot\rangle^{Z_{n}}\right)$. See [18] Lemma 5.2.7.5 for details.

(2) $\Phi_{n}$ is a tuple $\left(X, Y, \phi, \varphi_{-2, n}, \varphi_{0, n}\right)$, where $X, Y$ are $\mathcal{O}$-lattices that are isomorphic as $B$-modules after tensoring with $\mathbb{Q}$ and $\phi: Y \hookrightarrow X$ is an $\mathcal{O}$-invariant embedding.

$$
\varphi_{-2, n}: G r_{-2}^{Z_{n}} \stackrel{\sim}{\rightarrow} \operatorname{Hom}(X / n X,(\mathbb{Z} / n \mathbb{Z})(1))
$$

and

$$
\varphi_{0, n}: G r_{0}^{Z_{n}} \stackrel{\sim}{\rightarrow} Y / n Y
$$

are isomorphisms that are reduction modulo $n$ of $\mathcal{O}$-equivariant isomorphisms $\varphi_{-2}: G r_{-2}^{Z} \stackrel{\sim}{\rightarrow}$ $\operatorname{Hom}_{\hat{\mathbb{Z}}^{p}}\left(X \otimes \hat{\mathbb{Z}}^{p}, \hat{\mathbb{Z}}^{p}(1)\right)$ and $\varphi_{0}: G r_{0}^{Z} \stackrel{\sim}{\rightarrow} Y \otimes \hat{\mathbb{Z}}^{p}$ such that

$$
\varphi_{-2}(x)\left(\phi\left(\varphi_{0}(y)\right)\right)=\langle x, y\rangle
$$

for $x \in G r_{-2}^{Z}$ and $y \in G r_{0}^{Z}$.

(3) $\delta_{n}: \underset{i}{\oplus} G r_{i}^{Z_{n}} \stackrel{\sim}{\rightarrow} L / n L$ is a splitting that is reduction modulo $n$ of a splitting $\underset{i}{\oplus} G r_{i}^{Z} \stackrel{\sim}{\rightarrow} L \otimes \hat{\mathbb{Z}}^{p}$.

The tuple $\left(Z_{n}, \Phi_{n}, \delta_{n}\right)$ is called a cusp label at principal level $n$, and $\left[\left(Z_{n}, \Phi_{n}, \delta_{n}\right)\right]$ is the equivalence classes of the cusp label; see [18] Definition 5.4.1.9 for the precise definition of equivalences.

There is a precise description of closure relations of strata in terms of the cusp labels parametrising them; see [18] Definition 5.4.1.14 for details.

Remark 3.11. There are also toroidal compactifications of $M_{n}$ together with universal semi-abelian varieties over them that parametrise how abelian varieties degenerate into semi-abelian varieties. The toric part of the universal semi-abelian variety is parametrised by the cusp labels, which are discrete in nature. The minimal compactification is roughly obtained by contracting the isomorphic toric part, so it keeps track of only information on the abelian part, which is where the strata in Thoerem 3.10 come from.

In other words, the toric part of toroidal compactifications degenerates into discrete indexing sets of the strata, and the abelian part is remembered in the strata themselves. What is lost by passing to minimal compactifications is the extension between torus and abelian varieties.

Corollary 3.12. $M_{K(n)} / \Delta$ has a compactification $\left(M_{K(n)} / \Delta\right)^{\text {min }}$ together with a stratification by locally closed subschemes

$$
\begin{gathered}
\left(M_{K(n)} / \Delta\right)^{\min }=\coprod_{\substack{\alpha \in \Omega \\
\delta \in \Lambda}} M_{n}\left(L, \operatorname{Tr}_{\mathcal{O}_{F} / \mathbb{Z}} \circ\left(\alpha \delta\langle\cdot, \cdot\rangle_{F}\right)\right)^{\min } \\
\quad=\coprod_{\substack{\alpha \in \Omega \\
\delta \in \Lambda}} \coprod_{\left.\left(Z_{\alpha \delta, n}, \Phi_{\alpha \delta, n}, \delta_{\alpha \delta, n}\right)\right]} M_{n}\left(L^{Z_{\alpha \delta, n}},\langle\cdot, \cdot\rangle^{Z_{\alpha \delta, n}}\right)
\end{gathered}
$$


where $\left(Z_{\alpha \delta, n}, \Phi_{\alpha \delta, n}, \delta_{\alpha \delta, n}\right)$ are cusp labels of $M_{n}\left(L, \operatorname{Tr}_{\mathcal{O}_{F} / \mathbb{Z}} \circ\left(\alpha \delta\langle\cdot, \cdot\rangle_{F}\right)\right)$, and $M_{n}\left(L^{Z_{\alpha \delta, n}},\langle\cdot, \cdot\rangle^{Z_{\alpha \delta, n}}\right)$ are as in the theorem. See Warning 3.9 for clarifications.

\subsection{The partial Frobenius}

From now on, we assume that $p$ satisfies the following condition:

$p$ splits completely in the center $F^{c}$ of $B$.

This implies that $\mathcal{O} \otimes \mathbb{Z}_{p} \cong \prod M_{n_{i}}\left(\mathbb{Z}_{p}\right), G$ and $G^{*}$ splits over $\mathbb{Q}_{p}$ and $G\left(\mathbb{Q}_{p}\right)=\prod_{\mathfrak{p}_{i}} H\left(\mathbb{Q}_{p}\right)$, where $\mathfrak{p}_{i}$ are prime ideals of $F=F^{c, *=1}$ such that $p=\prod_{i} \mathfrak{p}_{i}$.

In this section, all moduli problems are defined over $\mathcal{O}_{F_{0}} \otimes_{\mathbb{Z}} \mathbb{F}_{p}$; that is, $M_{n}$ or $M_{K(n)}$ in this section denotes $M_{n} \times_{\mathcal{O}_{F_{0}} \otimes \mathbb{Z}_{(p)}}\left(\mathcal{O}_{F_{0}} \otimes \mathbb{F}_{p}\right)$ or $M_{K(n)} \times_{\mathcal{O}_{F_{0}} \otimes \mathbb{Z}_{(p)}}\left(\mathcal{O}_{F_{0}} \otimes \mathbb{F}_{p}\right)$ using notations in previous sections. We follow Nekovář's approach as in [25].

Definition 3.13. We fix a $\xi \in F_{+}^{\times}$satisfying $v_{\mathfrak{p}_{i}}(\xi)=1$ and $v_{\mathfrak{p}_{i^{\prime}}}(c)=0$ for $i^{\prime} \neq i$. The partial Frobenius $F_{\mathfrak{p}_{i}}: M_{K(n)} / \Delta \rightarrow M_{K(n)} / \Delta$ is defined by disoint union of maps

$$
M_{\alpha, K(n)} / \Delta \rightarrow M_{\alpha^{\prime}, K(n)} / \Delta
$$

sending $(A, \lambda, i, \overline{(\eta, u)})$ in Definition 3.6 to $\left(A^{\prime}, \lambda^{\prime}, i^{\prime}, \overline{\left(\eta^{\prime}, u^{\prime}\right)}\right){ }^{1}$, where

$$
A^{\prime}:=A /\left(\operatorname{Ker}(F)\left[\mathfrak{p}_{i}\right]\right)
$$

with $F$ the usual Frobenius and $\operatorname{Ker}(F)\left[\mathfrak{p}_{i}\right]:=\left\{x \in \operatorname{Ker}(F) \mid a x=0, \forall a \in \mathfrak{p}_{i}\right\}, i^{\prime}$ is induced by the quotient map $\pi_{\mathfrak{p}_{i}}: A \rightarrow A^{\prime}, \lambda^{\prime}$ is a prime to $p$ quasi-isogeny characterised by $\xi \lambda=\pi_{\mathfrak{p}_{i}}^{\vee} \circ \lambda^{\prime} \circ \pi_{\mathfrak{p}_{i}}$, $\eta^{\prime}=\pi_{\mathfrak{p}_{i}} \circ \eta$ and $\alpha^{\prime}$ is defined by

$$
\xi \alpha=\epsilon \alpha^{\prime} \lambda
$$

where $\alpha^{\prime} \in \Omega, \epsilon \in\left(\mathcal{O}_{F} \otimes \mathbb{Z}_{(p)}\right)_{+}^{\times}$and $\lambda \in\left(\mathcal{O}_{F} \otimes \hat{\mathbb{Z}}^{p}\right)^{\times}$as in decomposition (15). Lastly, $u^{\prime}$ is the composition of $\mathcal{O}_{F} \otimes \hat{\mathbb{Z}}^{p}$-equivariant isomorphisms

$$
u^{\prime}: \mathfrak{D}_{F}^{-1} \otimes \hat{\mathbb{Z}}^{p}(1) \stackrel{\lambda}{\sim} \mathfrak{D}_{F}^{-1} \otimes \hat{\mathbb{Z}}^{p}(1) \stackrel{u}{\sim} T^{p}\left(\mathfrak{D}_{F}^{-1} \otimes_{\mathbb{Z}} \mathbf{G}_{m, \bar{s}}\right) .
$$

Remark 3.14. It is easy to see that $F_{\mathfrak{p}_{i}}$ is independent of the choice of $\xi$. Moreover, the same definition works for $p$ not necessarily split in $F^{c}$. We make the assumption because that is the only case we will use.

We observe that

$$
F_{\mathfrak{p}_{i}} F_{\mathfrak{p}_{j}}=F_{\mathfrak{p}_{j}} F_{\mathfrak{p}_{i}}
$$

and

$$
\prod_{i} F_{\mathfrak{p}_{i}}=F
$$

where $\mathrm{F}$ is the usual Frobenius, explaining the name partial Frobenius.

It is helpful to write the partial Frobenius in terms of the decomposition (17). We will use the description to prove that the partial Frobenius extends to minimal compactifications and toroidal compactifications.

\footnotetext{
${ }^{1} A^{\prime}$ satisfies the determinant condition because $\operatorname{Lie}_{A^{\prime}}=\operatorname{Lie}_{A^{\prime}[p]}=\underset{j}{\oplus} \operatorname{Lie}_{A^{\prime}\left[\mathfrak{p}_{j}\right]}=\underset{j \neq i}{\oplus} \operatorname{Lie}_{A\left[\mathfrak{p}_{j}\right]} \oplus F^{*} \operatorname{Lie}_{A\left[\mathfrak{p}_{i}\right]}$ as $\mathcal{O} \otimes \mathbb{F} p^{-}$ modules, showing that $\mathrm{Lie}_{A^{\prime}}$ has the same $\mathcal{O} \otimes \mathbb{F}_{p}$ structure as $L_{i} e_{A}$, which satisfies the determinant condition by our choice.
} 
The equation

$$
\xi \alpha=\epsilon \alpha^{\prime} \lambda
$$

with $\alpha^{\prime} \in \Omega, \epsilon \in\left(\mathcal{O}_{F} \otimes \mathbb{Z}_{(p)}\right)_{+}^{\times}$and $\lambda \in\left(\mathcal{O}_{F} \otimes \hat{\mathbb{Z}}^{p}\right)^{\times}$plays an important role in the definition of the partial Frobenius. In particular, it determines how the partial Frobenius permutes components parametrised by $\alpha \in \Omega$ as in (11). We refine the description by using the finer decomposition (17) parametrised by $\alpha \delta$.

With notations as in the previous paragraph, let

$$
\lambda \delta=\epsilon_{0} \delta^{\prime} \gamma
$$

where $\delta, \delta^{\prime} \in \Lambda, \epsilon_{0} \in \mathcal{O}_{F,+}^{\times}$and $\gamma \in\left(v(K(n)) \hat{\mathbb{Z}}^{p, \times}\right)$, as in the decomposition (14). From equations (12) and (13), we observe that

$$
v(K(n)) \hat{\mathbb{Z}}^{p, \times}=\coprod_{\kappa} v(K(n)) \kappa
$$

where $\kappa$ ranges over a complete set of representatives of $\hat{\mathbb{Z}}^{p, \times} / v(\mathcal{U}(n)) \cong(\mathbb{Z} / n \mathbb{Z})^{\times}$in $\hat{\mathbb{Z}}^{p, \times}$. Let

$$
\gamma=\beta \kappa
$$

with $\beta \in v(K(n))$ and $\kappa$ as above be the decomposition of $\gamma$.

The partial Frobenius $F_{\mathfrak{p}_{i}}$ induces a map

$$
M_{n}\left(L, \operatorname{Tr}_{\mathcal{O}_{F} / \mathbb{Z}} \circ\left(\alpha \delta\langle\cdot, \cdot\rangle_{F}\right)\right) \rightarrow M_{n}\left(L, \operatorname{Tr}_{\mathcal{O}_{F} / \mathbb{Z}} \circ\left(\alpha^{\prime} \delta^{\prime}\langle\cdot, \cdot\rangle_{F}\right)\right)
$$

sending $\left(A, \lambda, i,\left(\alpha_{n}, v_{n}\right)\right)$ in Definition 3.5 to $\left(A^{\prime}, \lambda^{\prime}, i^{\prime},\left(\alpha_{n}^{\prime}, v_{n}^{\prime}\right)\right)$, where $A^{\prime}:=A /\left(\operatorname{Ker}(F)\left[\mathfrak{p}_{i}\right]\right), i^{\prime}$ is induced by the quotient map $\pi_{\mathfrak{p}_{i}}: A \rightarrow A^{\prime}, \lambda^{\prime}$ is characterised by $\xi \lambda=\pi_{\mathfrak{p}_{i}}^{\vee} \circ \lambda^{\prime} \circ \pi_{\mathfrak{p}_{i}}$, which defines a quasi-isogeny $\lambda^{\prime}, \alpha_{n}^{\prime}=\pi_{\mathfrak{p}_{i}} \circ \alpha_{n}$, and $v_{n}^{\prime}=v_{n} \circ \kappa$. In the last equation, we view $\kappa$ as an element of $(\mathbb{Z} / n \mathbb{Z})^{\times}$that acts on $\mathbb{Z} / n \mathbb{Z}(1)$, and $v_{n}^{\prime}$ is defined to be

$$
v_{n}^{\prime}:(\mathbb{Z} / n \mathbb{Z}(1))_{S} \underset{\sim}{\stackrel{\kappa}{\longrightarrow}}(\mathbb{Z} / n \mathbb{Z}(1))_{S} \stackrel{v_{n}}{\longrightarrow} \mu_{n, S}
$$

A subtle point in the above description is that in Definition $3.5, \lambda^{\prime}$ should not only be a prime to $p$ quasi-isogeny but an actual isogeny. The characterisation $\xi \lambda=\pi_{\mathfrak{p}_{i}}^{\vee} \circ \lambda^{\prime} \circ \pi_{\mathfrak{p}_{i}}$ defines a quasi-isogeny $\lambda^{\prime}$ but does not give an isogeny $\lambda^{\prime}$ a priori. We have to check that $\lambda^{\prime}$ is indeed a prime to p isogeny to make the above a well-defined map.

Before giving the proof, let us introduce some more suggestive notations. Let $A^{\left(\mathfrak{p}_{i}\right)}:=$ $A /\left(\operatorname{Ker}(F)\left[\mathfrak{p}_{i}\right]\right)$ and $F^{\left(\mathfrak{p}_{i}\right)}:=\pi_{\mathfrak{p}_{i}}: A \rightarrow A^{\left(\mathfrak{p}_{i}\right)}$. Then we observe that there is a natural map $V^{\left(\mathfrak{p}_{i}\right)}: A^{\left(\mathfrak{p}_{i}\right)} \rightarrow A \otimes_{\mathcal{O}_{F}} \mathfrak{p}_{i}^{-1}$ such that the composition

$$
A \stackrel{F^{\left(\mathfrak{p}_{i}\right)}}{\longrightarrow} A^{\left(\mathfrak{p}_{i}\right)} \stackrel{V^{\left(\mathfrak{p}_{i}\right)}}{\longrightarrow} A \otimes_{\mathcal{O}_{F}} \mathfrak{p}_{i}^{-1}
$$

is the map $i d_{A} \otimes_{\mathcal{O}_{F}}\left(\mathcal{O}_{F} \hookrightarrow \mathfrak{p}_{i}^{-1}\right)$, which has kernel $A\left[\mathfrak{p}_{i}\right]$. Here $\mathfrak{p}_{i}^{-1}$ is the inverse of $\mathfrak{p}_{i}$ as fractional ideals and $A \otimes_{\mathcal{O}_{F}} \mathfrak{p}_{i}^{-1}$ is defined in the category of fppf sheaf of $\mathcal{O}_{F}$-modules, which can be easily seen to be represented by an abelian scheme isogenous to $A$. Here $F^{\mathfrak{p}_{i}}$ and $V^{\mathfrak{p}_{i}}$ should be viewed as partial Frobenius and Verschiebung, whose products over all $i$ will be the usual ones. 
We have a commutative diagram

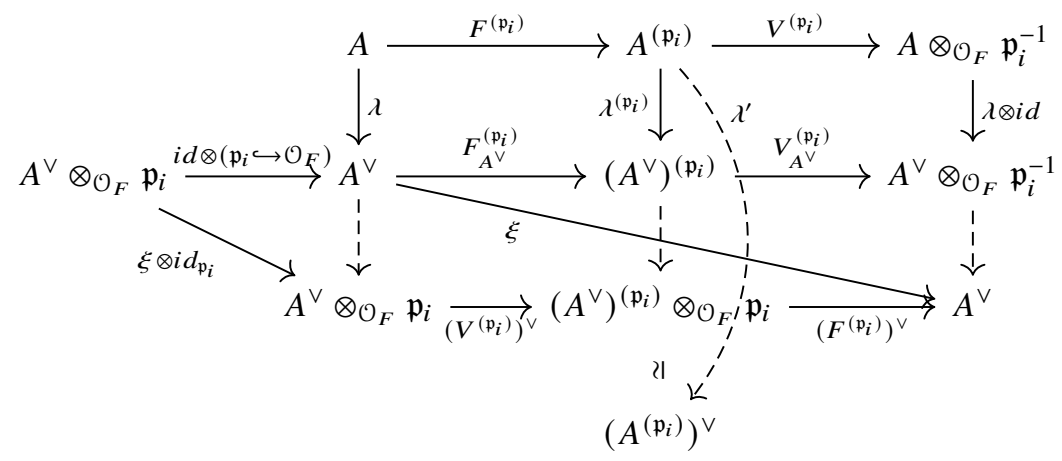

that induces the dashed arrows. For example, the left dashed arrow is induced by $A^{\vee}\left[\mathfrak{p}_{i}\right] \subset A^{\vee}[\xi]$, and similarly for the other two. We define $\lambda^{\prime}$ to be a composition of the middle vertical maps, which is an actual isogeny and satisfies $\xi \lambda=\pi_{\mathfrak{p}_{i}}^{\vee} \circ \lambda^{\prime} \circ \pi_{\mathfrak{p}_{i}}$ as the diagram shows.

The only nontrivial arrow in the above diagram is the isomorphism

$$
\left(A^{\left(\mathfrak{p}_{i}\right)}\right)^{\vee} \cong\left(A^{\vee}\right)^{\left(\mathfrak{p}_{i}\right)} \otimes_{\mathcal{O}_{F}} \mathfrak{p}_{i} .
$$

We give a proof here.

Lemma 3.15. With notations as above, for any abelian scheme A/S over a characteristic $p$ scheme $S$, together with a ring homomorphism $\mathcal{O} \rightarrow \operatorname{End}_{S}(A)$, we have a canonical isomorphism

$$
\left(A^{\left(\mathfrak{p}_{i}\right)}\right)^{\vee} \cong\left(A^{\vee}\right)^{\left(\mathfrak{p}_{i}\right)} \otimes_{\mathcal{O}_{F}} \mathfrak{p}_{i}
$$

Proof. Applying $\operatorname{Hom}_{f \text { p p } f}\left(-, \mathbf{G}_{m}\right)$ to the short exact sequence

$$
0 \rightarrow A\left[\mathfrak{p}_{i}\right] / \operatorname{Ker}(F)\left[\mathfrak{p}_{i}\right] \rightarrow A^{\left(\mathfrak{p}_{i}\right)} \stackrel{V^{\left(\mathfrak{p}_{i}\right)}}{\longrightarrow} A \otimes_{\mathcal{O}_{F}} \mathfrak{p}_{i}^{-1} \rightarrow 0
$$

and using that $\operatorname{Ext}_{f p p f}^{1}\left(A, \mathbf{G}_{m}\right) \cong A^{\vee}$, we have

$$
0 \rightarrow \operatorname{Hom}_{f p p f}\left(A\left[\mathfrak{p}_{i}\right] / \operatorname{Ker}(F)\left[\mathfrak{p}_{i}\right], \mathbf{G}_{m}\right) \rightarrow A^{\vee} \otimes_{\mathcal{O}_{F}} \mathfrak{p}_{i} \rightarrow\left(A^{\left(\mathfrak{p}_{i}\right)}\right)^{\vee} \rightarrow 0 .
$$

We know that $H_{\operatorname{lom}}\left(A\left[\mathfrak{p}_{i}\right] / \operatorname{Ker}(F)\left[\mathfrak{p}_{i}\right], \mathbf{G}_{m}\right)$ is the Cartier dual $\left(A\left[\mathfrak{p}_{i}\right] / \operatorname{Ker}(F)\left[\mathfrak{p}_{i}\right]\right)^{\vee}$ of $A\left[\mathfrak{p}_{i}\right] / \operatorname{Ker}(F)\left[\mathfrak{p}_{i}\right]$, so the dual of the short exact sequence

$$
0 \rightarrow \operatorname{Ker}(V) \rightarrow \operatorname{Ker}(V) \otimes_{\mathcal{O}_{F}} \mathfrak{p}_{i}^{-1} \rightarrow A\left[\mathfrak{p}_{i}\right] / \operatorname{Ker}(F)\left[\mathfrak{p}_{i}\right] \rightarrow 0
$$

gives

$$
\begin{gathered}
\left(A\left[\mathfrak{p}_{i}\right] / \operatorname{Ker}(F)\left[\mathfrak{p}_{i}\right]\right)^{\vee} \cong \operatorname{Ker}\left(\operatorname{Ker}(V)^{\vee} \otimes_{\mathcal{O}_{F}} \mathfrak{p}_{i} \rightarrow \operatorname{Ker}(V)^{\vee}\right) \\
\cong \operatorname{Ker}\left(\operatorname{Ker}\left(F_{A^{\vee}}\right) \otimes_{\mathcal{O}_{F}} \mathfrak{p}_{i} \rightarrow \operatorname{Ker}\left(F_{A^{\vee}}\right)\right) \\
\cong \operatorname{Ker}\left(F_{A^{\vee}}\right)\left[\mathfrak{p}_{i}\right] \otimes_{\mathcal{O}_{F}} \mathfrak{p}_{i}
\end{gathered}
$$

which is the kernel of

$$
A^{\vee} \otimes_{\mathcal{O}_{F}} \mathfrak{p}_{i} \stackrel{F^{\left(\mathfrak{p}_{i}\right)} \otimes i d}{\longrightarrow}\left(A^{\vee}\right)^{\left(\mathfrak{p}_{i}\right)} \otimes_{\mathcal{O}_{F}} \mathfrak{p}_{i}
$$

$\operatorname{proving}\left(A^{\left(\mathfrak{p}_{i}\right)}\right)^{\vee} \cong\left(A^{\vee}\right)^{\left(\mathfrak{p}_{i}\right)} \otimes_{\mathcal{O}_{F}} \mathfrak{p}_{i}$. 
We now state the main technical result of the article, which claims that the partial Frobenius extends to the minimal compactification in Corallary 3.12 .

Theorem 3.16. $F_{\mathfrak{p}_{i}}$ extends to a morphism

$$
F_{\mathfrak{p}_{i}}:\left(M_{K(n)} / \Delta\right)^{\text {min }} \longrightarrow\left(M_{K(n)} / \Delta\right)^{\text {min }}
$$

sending the strata $M_{n}\left(L^{Z_{\alpha \delta, n}},\langle\cdot, \cdot\rangle^{Z_{\alpha \delta, n}}\right)$ associated to $\alpha \in \Omega, \delta \in \Lambda$ and the cusp label $\left[\left(Z_{\alpha \delta, n}, \Phi_{\alpha \delta, n}, \delta_{\alpha \delta, n}\right)\right]$ to the strata $M_{n}\left(L^{\left.Z_{\alpha^{\prime} \delta^{\prime}, n},\langle\cdot, \cdot\rangle^{Z_{\alpha^{\prime} \delta^{\prime}, n}}\right)}\right.$ associated to $\alpha^{\prime} \in \Omega, \delta^{\prime} \in \Lambda$ as in the above description of the partial Frobenius, and the cusp label is $\left[\left(Z_{\alpha^{\prime} \delta^{\prime}, n}, \Phi_{\alpha^{\prime} \delta^{\prime}, n}, \delta_{\alpha^{\prime} \delta^{\prime}, n}\right)\right]$ defined as follows:

$$
Z_{\alpha^{\prime} \delta^{\prime}, n}=Z_{\alpha \delta, n}
$$

If $\Phi_{\alpha \delta, n}=\left(X, Y, \phi, \varphi_{-2, n}, \varphi_{0, n}\right)$, then

$$
\Phi_{\alpha^{\prime} \delta^{\prime}, n}=\left(X \otimes_{\mathcal{O}_{F}} \mathfrak{p}_{i}, Y, \phi^{\prime}, \varphi_{-2, n}^{\prime}, \varphi_{0, n}^{\prime}\right),
$$

where

$$
\begin{gathered}
\varphi_{-2, n}^{\prime}: G r_{-2}^{Z_{\alpha^{\prime} \delta^{\prime}, n}}=G r_{-2}^{Z_{\alpha \delta, n}} \stackrel{\varphi_{-2, n}}{\longrightarrow} \operatorname{Hom}(X / n X,(\mathbb{Z} / n \mathbb{Z})(1)) \\
\stackrel{\sim}{\longrightarrow} \operatorname{Hom}\left(X \otimes \mathfrak{p}_{i} / n\left(X \otimes \mathfrak{p}_{i}\right),(\mathbb{Z} / n \mathbb{Z})(1)\right)
\end{gathered}
$$

and

$$
\varphi_{0, n}^{\prime}: G r_{0}^{Z_{\alpha^{\prime} \delta^{\prime}, n}}=G r_{0}^{Z_{\alpha \delta, n}} \stackrel{\varphi_{0, n}}{\longrightarrow} Y / n Y
$$

Lastly, $\phi^{\prime}$ is defined by the following diagram similar to the above diagram defining $\lambda^{\prime}$ :

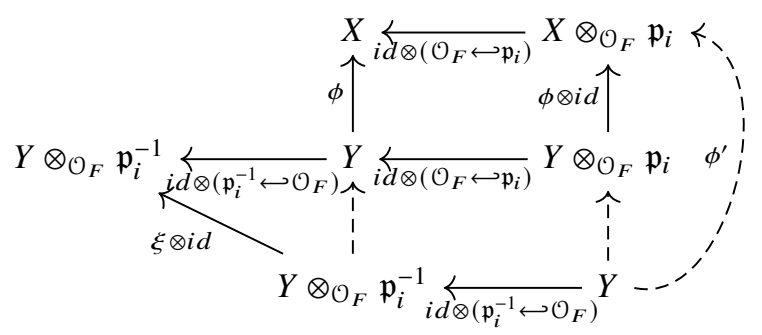

Moreover, on each strata, $F_{\mathfrak{p}_{i}}$ induces the morphism

$$
M_{n}\left(L^{Z_{\alpha \delta, n}},\langle\cdot, \cdot\rangle^{Z_{\alpha \delta, n}}\right) \rightarrow M_{n}\left(L^{Z_{\alpha^{\prime} \delta^{\prime}, n}},\langle\cdot, \cdot\rangle^{Z_{\alpha^{\prime} \delta^{\prime}, n}}\right)
$$

sending $\left(A, \lambda, i,\left(\alpha_{n}, v_{n}\right)\right)$ to $\left(A^{\prime}, \lambda^{\prime}, i^{\prime},\left(\alpha_{n}^{\prime}, v_{n}^{\prime}\right)\right)$ as in the description before the theorem. For completeness, we summarise the description as follows. Using the above notations, $A^{\prime}:=A /\left(\operatorname{Ker}(F)\left[\mathfrak{p}_{i}\right]\right), i^{\prime}$ is induced by the quotient map $\pi_{\mathfrak{p}_{i}}: A \rightarrow A^{\prime}, \lambda^{\prime}$ is characterised by $\xi \lambda=\pi_{\mathfrak{p}_{i}}^{\vee} \circ \lambda^{\prime} \circ \pi_{\mathfrak{p}_{i}}$ which defines a prime to $p$ isogeny $\lambda^{\prime}, \alpha_{n}^{\prime}=\pi_{\mathfrak{p}_{i}} \circ \alpha_{n}$ and $\nu_{n}^{\prime}=v_{n} \circ \kappa$. In other words, restriction of the partial Frobenius to (suitable union of) strata recovers the partial Frobenius on them.

Remark 3.17. The diagram defining $\phi^{\prime}$ is similar to the diagram defining $\lambda^{\prime}$, and there is a reason for that. We will see in the proof that the diagram defining $\lambda^{\prime}$ also defines a polarisation for the universal semi-abelian variety over toroidal compactfications, and the diagram for $\phi^{\prime}$ is the one induced on the (character group of) toric part.

Moreover, the theorem is proved by first extending $F_{\mathfrak{p}_{i}}$ to toroidal compactifications and then contracting to a morphism on the minimal compactification. The description of the morphism on strata is obtained by looking at how $F_{\mathfrak{p}_{i}}$ operates on semi-abelian varieties. In particular, the morphism on 
indexing sets is obtained by looking at the toric part, and the morphism on strata is determined by the abelian part. See also Remark 3.11.

Remark 3.18. The description of strata in the minimal compactification shows that minimal compactification is plectic, which is the underlying reason that partial Frobenius extends to the minimal compactification. This can be made precise if we take care of the subtlety in dimension zero, which means taking appropriate unions of the strata to define Shimura varieties of similitude PEL type as in Definition 3.6 (the strata we use are of Kottwitz's type as in definition 3.5).

Another way to see the phenomenon is through Pink's mixed Shimura varieties, where he uses mixed Shimura varieties associated to parabolic subgroups (more precisely, the Levi group) to define strata of the minimal boundary, called rational boundary components in his terminology. In our case, the Shimura variety is associated to $G=\operatorname{Res}_{F / \mathbb{Q}} H$, and the parabloics are also of the form $\operatorname{Res}_{F / \mathbb{Q}} P$. However, the strata are assocaited to a subgroup $P_{1}$, in Pink's notation, of the parabolic, which is the Hermitian part in classical language, and this is not necessarily plectic; that is, not of the form $\operatorname{Res}_{F / \mathbb{Q}}(-)$. The reason is that in [28] Section 4.7, Pink defines $P_{1}$ as the group satisfying certain minimality property; see [28] Example 12.21 for an example how this kills 'plecticity'. The failure is similar to the difference between $G$ and $G^{*}$, and the remedy is the same. We can replace $P_{1}$ by another group in the parabolic, which is different only up to a similitude. Pink's theory still works in this slightly different setting, as already observed by him in Remark (ii) of [28] Section 4.11.

The proof of the theorem is rather technical, and we defer to the last section for details. We first give an application of it on the construction of plectic weight filtration of cohomology of Hilbert modular varieties in the next section.

\section{Hilbert modular varieties}

\subsection{Basics}

We now specialise discussions in the previous section to the Hilbert modular varieties. The notations in this section will be the same as in the previous one. We simply restrict everything to a special case as follows.

We take $\mathcal{O}=\mathcal{O}_{F}$, with $F$ a totally real field of degree $[F: \mathbb{Q}]=d$ and $*=i d$, which coincides with notations in the previous section in that $B=F$ and $F=F^{c}$ is the $*$-invariant part of the center of $B$. Moreover, $L=\mathcal{O}_{F} \oplus \mathcal{O}_{F},\langle\cdot, \cdot\rangle_{F}$ is the standard $\mathcal{O}_{F}$-bilinear alternating pairing defined by the matrix $\left(\begin{array}{cc}0 & -1 \\ 1 & 0\end{array}\right)$, and $\langle\cdot, \cdot\rangle=\operatorname{Tr}_{\mathcal{O}_{F} / \mathbb{Z}}\left(\langle\cdot, \cdot\rangle_{F}\right)$. The morphism $h: \mathbb{C} \rightarrow \operatorname{End}_{\mathcal{O}_{\mathbb{R}}}\left(L_{\mathbb{R}}\right)$ is defined by $h(x+i y)=\prod_{\tau: F \hookrightarrow \mathbb{R}}\left(\begin{array}{cc}x & -y \\ y & x\end{array}\right)$. These data define a type $\mathbf{C}$ PEL datum. It is easy to see that the reflex field $F_{0}$ is $\mathbb{Q}$. The relevant groups are

$$
G=\operatorname{Res}_{\mathcal{O}_{F} / \mathbb{Z}} G L_{2}
$$

and

$$
G^{*}=\operatorname{det}^{-1}\left(\mathbf{G}_{m}\right) \subset G,
$$

where det $: G \rightarrow \operatorname{Res}_{\mathcal{O}_{F} / \mathbb{Z}} \mathbf{G}_{m}$ is the similitude map.

We give a brief account of the moduli problem it defines, which is a special case of Definition 3.6. Let $\alpha \in \Omega$ be as in decomposition (15), then $M_{\alpha, K(n)}$ is the moduli space representing the functor associating a locally Noetherian $\mathbb{Z}_{(p)}$-scheme $S$ to the isomorphism classes of tuples $(A, \lambda, i,(\eta, u))$. Here $A$ is an abelian scheme over $S, \lambda: A \rightarrow A^{\vee}$ is a prime to $p$ polarisation and $i: \mathcal{O}_{F} \rightarrow E n d_{S}(A)$ a ring isomorphism inducing the trivial involution on $\mathcal{O}_{F}$ through $\lambda$ and a rank $1 \mathcal{O}_{F}$-module structure on Lie $(A)$. Note that the last condition is Kottwitz's determinant condition in this special case. Moreover, 
the level structure $(\eta, u)$ is an $\pi_{1}(S, \bar{s})$-invariant $K(n)$-orbit of $\mathcal{O}_{F} \otimes \mathbb{A}^{p \infty}$-equivariant isomorphism

$$
\eta: L \otimes \mathbb{A}^{p \infty}=\left(\mathcal{O}_{F} \otimes \mathbb{A}^{p \infty}\right)^{\oplus 2} \stackrel{\sim}{\rightarrow} V^{p} A_{\bar{s}}
$$

together with an $\mathcal{O}_{F} \otimes \hat{\mathbb{Z}}^{p}$-equivariant isomorphism

$$
u: \mathfrak{D}_{F}^{-1} \otimes \hat{\mathbb{Z}}^{p}(1) \stackrel{\sim}{\rightarrow} T^{p}\left(\mathfrak{D}_{F}^{-1} \otimes_{\mathbb{Z}} \mathbf{G}_{m, \bar{s}}\right)
$$

such that

$$
\langle\eta(x), \eta(y)\rangle_{\lambda}=\operatorname{Tr}_{\mathcal{O}_{F} / \mathbb{Z}}\left(u \circ\left(\alpha\langle x, y\rangle_{F}\right)\right)
$$

where $x, y \in L \otimes \mathbb{A}^{p \infty}$. Because we work only with principal level- $n$ structures, the level structure can also be seen as isomorphisms

$$
\left(\mathcal{O}_{F} / n \mathcal{O}_{F}\right)^{\oplus 2} \cong A[n]
$$

and

$$
\mathfrak{d}_{F}^{-1} / n \mathfrak{d}_{F}^{-1} \cong \mathfrak{d}_{F}^{-1} \otimes \mu_{n}
$$

Remark 4.1. In the literature, it is common to use a variant of the above moduli problem. More precisely, the polarisation is defined as an $\mathcal{O}_{F}$-equivariant isomorphism

$$
\left(\mathfrak{c}, \mathfrak{c}_{+}\right) \cong\left(\operatorname{Hom}_{\mathcal{O}_{F}}^{\operatorname{Sym}}\left(A, A^{\vee}\right), \operatorname{Hom}_{\mathcal{O}_{F}}^{\operatorname{Sym}}\left(A, A^{\vee}\right)_{+}\right)
$$

where $\mathfrak{c}$ is a fixed prime to $p$ fractional ideal representing $[\alpha] \in C l_{+}(F)=\left(\mathcal{O}_{F} \otimes \mathbb{Z}_{(p)}\right)_{+}^{\times} \backslash(F \otimes$ $\left.\mathbb{A}^{(p, \infty)}\right)^{\times} /\left(\mathcal{O}_{F} \otimes \hat{\mathbb{Z}}^{p}\right)^{\times}, \mathfrak{c}_{+}$is the totally positive part (the elements that are positive for all embeddings of $F$ into $\mathbb{R}), \operatorname{Hom}_{\mathcal{O}_{F}}^{\operatorname{Sym}}\left(A, A^{\vee}\right)$ is the symmetric $\mathcal{O}_{F}$-equivariant homomorphisms and $\operatorname{Hom}_{\mathcal{O}_{F}}^{\operatorname{Sym}}\left(A, A^{\vee}\right)_{+}$is the set of polarisations. The level structure is defined as an $\mathcal{O}_{F}$-equivariant isomorphism $\left(\mathcal{O}_{F} / n \mathcal{O}_{F}\right)^{\oplus 2} \cong$ $A[n]$ together with an isomorphism $\mathcal{O}_{F} / n \mathcal{O}_{F} \cong \mu_{n} \otimes \mathrm{c}^{*}$; see [30] for details. For the equivalence to our definition, see [14] Section 4.1.1 for some discussion.

Similar to the previous section, we have

$$
S h_{K(n)}(G, h)=\coprod_{\alpha \in \Omega} M_{\alpha, K(n)} / \Delta=M_{K(n)} / \Delta,
$$

where $M_{K(n)}:=\coprod_{\alpha \in \Omega} M_{\alpha, K(n)}$, and

$$
M_{K(n)} / \Delta=\coprod_{\substack{\alpha \in \Omega \\ \delta \in \Lambda}} M_{n}\left(L, \operatorname{Tr}_{\mathcal{O}_{F} / \mathbb{Z}} \circ\left(\alpha \delta\langle\cdot, \cdot\rangle_{F}\right)\right)
$$

We now describe the minimal compactification in more explicit terms. Recall from Theorem 3.12 that cusp labels are equivalence classes of tuples $\left[\left(Z_{n}, \Phi_{n}, \delta_{n}\right)\right]$, where $Z_{n}$ is an $\mathcal{O}_{F}$-invariant filtration

$$
0 \subset Z_{n,-2} \subset Z_{n,-1} \subset Z_{n, 0}=L / n L
$$

on $L / n L$ satisfying $Z_{n,-2}^{\perp}=Z_{n,-1}$ and some liftability condition, $\Phi_{n}$ is a tuple $\left(X, Y, \phi, \varphi_{-2, n}, \varphi_{0, n}\right)$ and $\delta_{n}: \underset{i}{\oplus} G r_{i}^{Z_{n}} \stackrel{\sim}{\rightarrow} L / n L$ is a splitting with a liftability condition. In the definition of $\Phi_{n}, X, Y$ are $\mathcal{O}$-lattices that are isomorphic as $B$-modules after tensoring with $\mathbb{Q}, \phi: Y \hookrightarrow X$ is an $\mathcal{O}$-invariant embedding.

$$
\varphi_{-2, n}: G r_{-2}^{Z_{n}} \stackrel{\sim}{\rightarrow} \operatorname{Hom}(X / n X,(\mathbb{Z} / n \mathbb{Z})(1))
$$


and

$$
\varphi_{0, n}: G r_{0}^{Z_{n}} \stackrel{\sim}{\rightarrow} Y / n Y
$$

are isomorphisms that are reduction modulo $\mathrm{n}$ of $\mathcal{O}$-equivariant isomorphisms $\varphi_{-2}: G r_{-2}^{Z} \stackrel{\sim}{\rightarrow}$ $\operatorname{Hom}_{\hat{\mathbb{Z}}^{p}}\left(X \otimes \hat{\mathbb{Z}}^{p}, \hat{\mathbb{Z}}^{p}(1)\right)$ and $\varphi_{0}: G r_{0}^{Z} \stackrel{\sim}{\rightarrow} Y \otimes \hat{\mathbb{Z}}^{p}$ such that

$$
\varphi_{-2}(x)\left(\phi\left(\varphi_{0}(y)\right)\right)=\langle x, y\rangle
$$

for $x \in G r_{-2}^{Z}$ and $y \in G r_{0}^{Z}$.

In our case, $L=\mathcal{O}_{F}^{\oplus 2}$ and there are essentially two different filtrations on $L / n L$, either $Z_{n,-2}=0$ and $Z_{n,-1}=L / n L$ or $Z_{n,-2}=Z_{n,-1}$ is a $\mathcal{O}_{F}$-submodule of $L / n L$ being reduction of a rank $1 \mathcal{O}_{F} \otimes \mathbb{A}^{p}$ submodule of $L \otimes \mathbb{A}^{p}$. The first case is trivial; the corresponding strata is the open strata in the minimal compactification. We focus on the second case from now on.

The isomorphisms $\varphi_{0, n}$ and $\varphi_{-2, n}$ force $X$ and $Y$ to be rank $1 \mathcal{O}_{F}$-modules, which are isomorphic to fractional ideals of $F$ and classified by $C l(F)$. We observe that $G r_{-1}^{Z_{n}}=0$, implying that $L^{Z_{n}}=0$. Thus, the strata associated to $\left[\left(Z_{n}, \Phi_{n}, \delta_{n}\right)\right]$ must be $\operatorname{Isom}\left(\mathbb{Z} / n \mathbb{Z}(1), \mu_{n}\right)$; that is,

$$
M_{n}\left(L^{Z_{n}},\langle\cdot, \cdot\rangle^{Z_{n}}\right)=\operatorname{Isom}\left(\mathbb{Z} / n \mathbb{Z}(1), \mu_{n}\right) ;
$$

see Remark 3.4 for explanations. In other words, the boundary components all have dimension zero, and they are generally referred to as cusps.

\subsection{The weight spectral sequence}

We now make the spectral sequence in Theorem 2.14 more explicit in our special case. With notations as in Subsection 2.2, we take $V=\mathbb{Q}(0)$ to be the trivial representation of $G$, then $\mathcal{F} V=\mathbb{Q}(0)$ is the constant sheaf in $D_{m}^{b}\left(S h_{K(n)}(G, h)\right)$; that is, $\mathbb{Q}(0)$ is either the constant Hodge module $\mathbb{Q}^{H}(0)$ or the constant mixed $l$-adic sheaf $\overline{\mathbb{Q}_{l}}(0)$. Let $j: S h_{K(n)}(G, h) \hookrightarrow S h_{K(n)}(G, h)^{\text {min }}$ be the open embedding, then the spectral sequence is

$$
E_{1}^{p, q}=\mathbb{H}^{p+q}\left(S h_{K(n)}(G, h)^{\min }, w_{\geq-p} w_{\leq-p} R j_{*}(\mathbb{Q}(0))\right) \Rightarrow H^{p+q}\left(S h_{K(n)}(G, h), \mathbb{Q}(0)\right) .
$$

Because $\mathbb{Q}(0)$ is pure of weight 0, Proposition 2.7 and Theorem 2.8 tell us that the first nontrivial piece is

$$
w_{\geq 0} w_{\leq 0} R j_{*}(\mathbb{Q}(0))=w_{\leq 0} R j_{*}(\mathbb{Q}(0))=j_{! *}(\mathbb{Q}(0))
$$

as we see in the discussion prior to Theorem 2.14, so

$$
E_{1}^{0, q}=I H^{q}\left(S h_{K(n)}(G, h)^{\min }, \mathbb{Q}(0)\right) .
$$

Similarly, the discussion before Theorem 2.14 gives information on the rest of the pieces. In particular, equation (4) tells that for $k>0$,

$$
w_{\leq k} w_{\geq k} R j_{*}(\mathbb{Q}(0))=i_{*} w_{\leq k} w_{\geq k} i^{*} R j_{*}(\mathbb{Q}(0)),
$$

where

$$
\begin{gathered}
i: S h_{K(n)}(G, h)^{\min } \backslash S h_{K(n)}(G, h)=\bigsqcup_{\substack{\alpha \in \Omega \\
\delta \in \Lambda}} \coprod_{\substack{\left.\left.Z_{\alpha \delta, n}, \Phi_{\alpha \delta, n}, \delta_{\alpha \delta, n}\right)\right] \\
Z_{\alpha \delta, n,-2} \neq 0}} \operatorname{Isom}\left(\mathbb{Z} / n \mathbb{Z}(1), \mu_{n}\right) \\
\hookrightarrow S h_{K(n)}(G, h)^{\text {min }}
\end{gathered}
$$


is the inclusion of the complement of $\operatorname{Sh}_{K(n)}(G, h)$; that is, the inclusion of finitely many cusps. Now Pink ([29]) or Burgos and Wildeshaus's ([5]) formula tells us that

$$
i_{\partial}^{*} R^{n} j_{*}(\mathbb{Q}(0))=\underset{a+b=n}{\oplus} \mathcal{F}\left(H^{a}\left(\bar{H}_{C}, H^{b}\left(\text { LieW }_{1}, \mathbb{Q}(0)\right)\right)\right)
$$

where $\partial:=\left[\left(Z_{\alpha \delta, n}, \Phi_{\alpha \delta, n}, \delta_{\alpha \delta, n}\right)\right]$ and $i_{\partial}$ is the inclusion of the cusp corresponding to $\partial$. Moreover, $W_{1}$ is the unipotent of the Borel subgroup corresponding to the cusp $\partial$, and $\bar{H}_{C}$ is an arithmetic subgroup of the linear part of the Levi group determined by the level $K(n)$. By proof of Theorem 3.5 in [31], we have that

$$
i_{\partial}^{*} R^{n} j_{*}(\mathbb{Q}(0))= \begin{cases}\bigwedge^{n}\left(\mathbb{Q}(0)^{d-1}\right) & 0 \leq n \leq d-1 \\ \bigwedge^{2 d-1-n}\left(\mathbb{Q}(0)^{d-1}\right)(-d) & d \leq n \leq 2 d-1\end{cases}
$$

Note that the author only works with the Hodge module case in [31], but the proof works equally well for the $l$-adic case. Indeed, if we view $\mathbb{Q}(0)$ as the trivial representation of $\mathbf{G}_{m}$, which is the group corresponding to the 0 -dimensional Shimura variety $\operatorname{Isom}\left(\mathbb{Z} / n \mathbb{Z}(1), \mu_{n}\right)$ indexed by $\partial$, and $(-d)$ twisting by $d$ th power of the dual of the standard representation, then the proof in [31] shows that

$$
i_{\partial}^{*} R^{n} j_{*}(\mathbb{Q}(0))= \begin{cases}\mathcal{F}\left(\bigwedge^{n}\left(\mathbb{Q}(0)^{d-1}\right)\right) & 0 \leq n \leq d-1 \\ \mathcal{F}\left(\bigwedge^{2 d-1-n}\left(\mathbb{Q}(0)^{d-1}\right)(-d)\right) & d \leq n \leq 2 d-1\end{cases}
$$

We will use a different parametrisation of the cusps than (20). Recall that $\Lambda$ in (14) is chosen such that

$$
\operatorname{Isom}_{\mathcal{O}_{F}}\left(\mathfrak{D}_{F}^{-1} / n \mathfrak{D}_{F}^{-1}(1), \mathfrak{D}_{F}^{-1} \otimes_{\mathbb{Z}} \mu_{n}\right)=\coprod_{\delta \in \Lambda} \operatorname{Isom}\left(\mathbb{Z} / n \mathbb{Z}(1), \mu_{n}\right) .
$$

We use it to rewrite (20) as

$$
i: S h_{K(n)}(G, h)^{\min } \backslash S h_{K(n)}(G, h)=\coprod_{\alpha \in \Omega} \coprod_{\partial} \operatorname{Isom}_{\mathcal{O}_{F}}\left(\mathfrak{D}_{F}^{-1} / n \mathfrak{D}_{F}^{-1}(1), \mathfrak{D}_{F}^{-1} \otimes_{\mathbb{Z}} \mu_{n}\right)
$$

with a new parametrisation set of cusps, which we still denote by $\partial$. For a precise description of $\partial$, see [10]. For such a parametrisation, the boundary is a union of 0-dimensional Shimura varieties associated to $\operatorname{Res}_{F / \mathbb{Q}} \mathbf{G}_{m}$, and a minor modification of the proof in [31] shows that

$$
i_{\partial}^{*} R^{n} j_{*}(\mathbb{Q}(0))= \begin{cases}\mathcal{F}\left(\bigwedge^{n}\left(\mathbb{Q}(0)^{d-1}\right)\right) & 0 \leq n \leq d-1 \\ \mathcal{F}\left(\bigwedge^{2 d-1-n}\left(\mathbb{Q}(0)^{d-1}\right)(-d)\right) & d \leq n \leq 2 d-1\end{cases}
$$

where $\partial$ denotes the cusps in (23), and $(-d)$ is twisting by the 1-dimensional representation $N m_{F / Q}^{-1}$ : $\operatorname{Res}_{F / \mathbb{Q}} \mathbf{G}_{m} \rightarrow \mathbf{G}_{m}$ of $\operatorname{Res}_{F / \mathbb{Q}} \mathbf{G}_{m}$. Note that the corresponding sheaf is the $(-d)$ th power of the Tate twisting sheaf, explaining the notation. Further, this is the only new observation one needs in the proof of the above.

Then together with equation (19) we have that for $k>0$,

$$
w_{\geq k} w_{\leq k} R^{n} j_{*}(\mathbb{Q}(0))= \begin{cases}i_{*}\left(\bigwedge^{2 d-1-n}\left(\mathbb{Q}(0)^{d-1}\right)(-d)\right) & d \leq n \leq 2 d-1, k=2 d \\ 0 & \text { otherwise }\end{cases}
$$


Thus, the spectral sequence (18) becomes

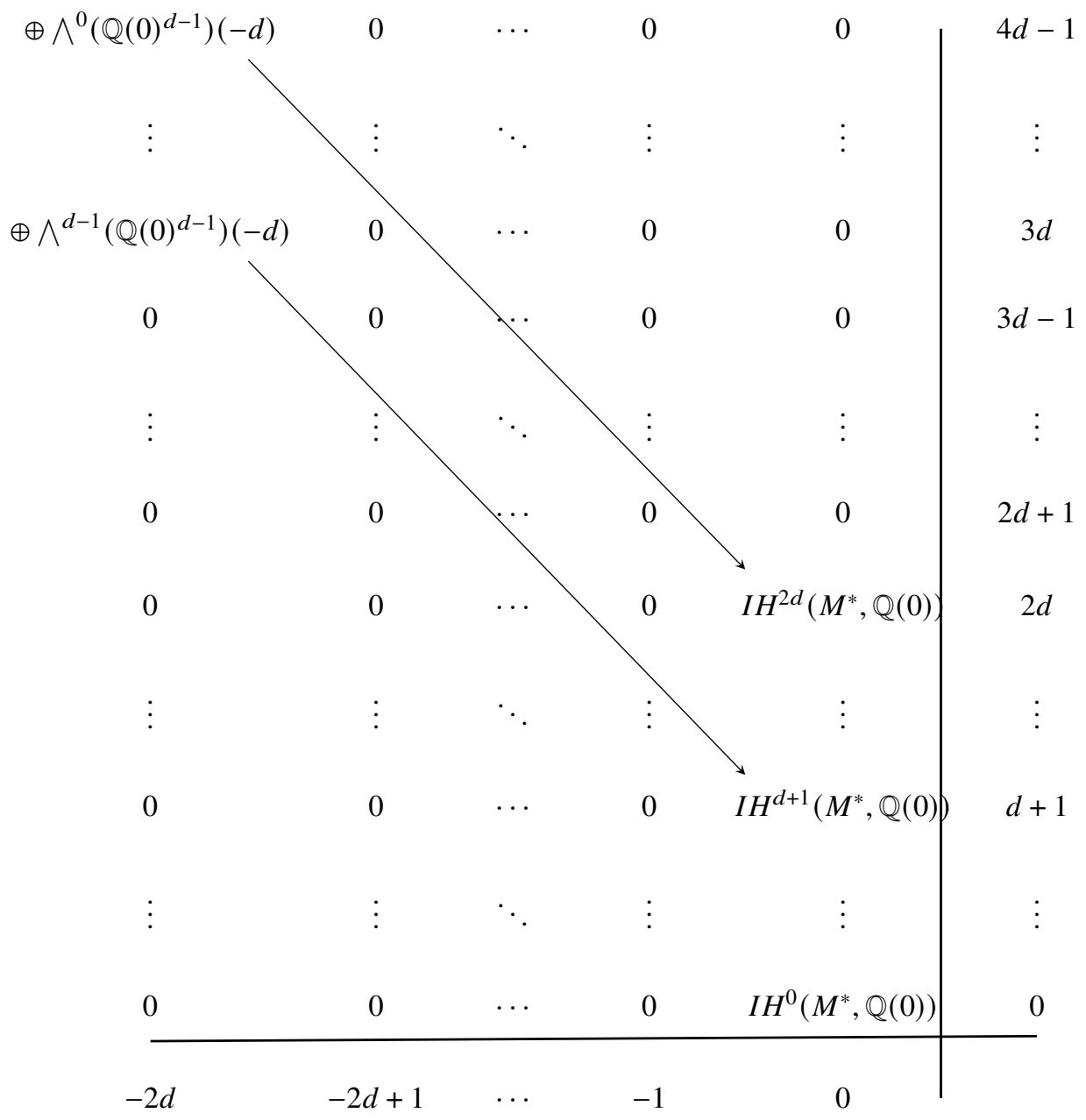

where

$M^{*}:=S h_{K(n)}(G, h)^{\min }, M:=S h_{K(n)}(G, h)$ and

$$
\begin{aligned}
\oplus \bigwedge^{i}\left(\mathbb{Q}(0)^{d-1}\right)(-d) & :=\underset{\partial}{\oplus}\left(\bigwedge^{i}\left(\mathbb{Q}(0)^{d-1}\right)(-d)\right)^{\oplus n} \\
= & H^{2 d-1-i}\left(M^{*} \backslash M, i^{*} R j^{*}(\mathbb{Q}(0))\right)
\end{aligned}
$$

for $0 \leq i \leq d-1$. Note that in either case (Hodge modules or $l$-adic), the cohomology is taken after passing to the algebraic closure of the base field, so $M^{*} \backslash M=\coprod_{\partial} \operatorname{Isom}\left(\mathbb{Z} / n \mathbb{Z}(1), \mu_{n}\right)$ becomes $\bigsqcup_{\partial} \coprod_{n}\{*\}$, explaining the second equality of the above.

Now we can read off from the above computation that

$$
\begin{gathered}
0 \longrightarrow E_{\infty}^{-2 d, 4 d-1} \longrightarrow \underset{\partial}{\oplus}\left(\bigwedge^{0}\left(\mathbb{Q}(0)^{d-1}\right)(-d)\right)^{\oplus n} \longrightarrow I H^{2 d}\left(M^{*}, \mathbb{Q}(0)\right) \longrightarrow E_{\infty}^{0,2 d} \longrightarrow 0 \\
0 \longrightarrow I H^{2 d-1}\left(M^{*}, \mathbb{Q}(0)\right) \longrightarrow H^{2 d-1}(M, \mathbb{Q}(0)) \longrightarrow E_{\infty}^{-2 d, 4 d-1} \longrightarrow 0,
\end{gathered}
$$


where $E_{\infty}^{0,2 d}=H^{2 d}(M, \mathbb{Q}(0))=0$ as $M$ is nonproper of dimension $d$. Moreover, we observe easily that

$$
H^{i}(M, \mathbb{Q}(0))=I H^{i}\left(M^{*}, \mathbb{Q}(0)\right)
$$

for $0 \leq i \leq d-1$, and

$$
0 \longrightarrow I H^{i}\left(M^{*}, \mathbb{Q}(0)\right) \longrightarrow H^{i}(M, \mathbb{Q}(0)) \longrightarrow \underset{\partial}{\oplus}\left(\bigwedge^{2 d-1-i}\left(\mathbb{Q}(0)^{d-1}\right)(-d)\right)^{\oplus n} \longrightarrow 0
$$

for $d \leq i \leq 2 d-2$. In the last exact sequence, we use that

$$
E_{\infty}^{-2 d, 2 d+i}=\underset{\partial}{\oplus}\left(\bigwedge^{2 d-1-i}\left(\mathbb{Q}(0)^{d-1}\right)(-d)\right)^{\oplus n}
$$

for $d \leq i \leq 2 d-2$, which follows because the domain and codomain of the differentials in the picture have different weights in this range.

We observe from the above computation that the spectral sequence (18) gives us the weight filtration on $H^{*}(M, \mathbb{Q}(0))$, which provides a new computation of the weight filtration of the cohomology of Hilbert modular varieties without using the Borel-Serre compactifications as done, for example, in the last section of [27]. This is a philosophically better computation because it is performed in the algebraic category, whereas the older computation uses the nonalgebraic Borel-Serre compactifications and proceeds in a more indirect way when establishing the mixed Hodge structures. See [1] for a modern treatment of the motivic meaning of the reductive Borel-Serre compactifications.

\subsection{The plectic weight filtration}

Now we make use of the spectral sequence (18) to construct the plectic weight filtration. Note that the filtration induced by (18) is a $\mathbb{Z}$-filtration, but the plectic weight filtration we are looking for is a $\mathbb{Z}^{d}$ filtration. We will use the partial Frobenius to cut out the $\mathbb{Z}$-filtration into a $\mathbb{Z}^{d}$-filtration and show that this is the sought-after plectic weight filtration.

Firstly, we compute the eigenvalues of the partial Frobenius on the boundary cohomology $H^{*}\left(M^{*} \backslash\right.$ $\left.M, i^{*} R j_{*}(\mathbb{Q}(0))\right)$. We denote the canonical PEL (up to similitude) smooth integral model $M_{K(n)} / \Delta$ of $M$ by $\mathscr{M}$, which is defined over an open dense subset of $\operatorname{Spec}(\mathbb{Z})$. Similarly, $\mathscr{M}^{*}$ is the integral model of the minimal compactification. Now choose a prime $p$ in the open subset such that it is split in $F$ and lies in the applicable range of Theorem 2.16. Then as we have already seen, the Frobenius $F_{r o b}$ on $\mathscr{M}_{\mathbb{F}_{p}}^{*}$ decomposes into $\operatorname{Frob}_{p}=\prod_{i} F_{i}$, where $F_{i}$ is the partial Frobenius corresponding to the prime $\mathfrak{p}_{i}$ in the prime decomposition $p=\prod_{i}^{i} \mathfrak{p}_{i}$ of $p$ in $F$.

Let us recall the construction of the $l$-adic sheaf on a Shimura variety coming from an algebraic representation, following Pink ([29]). Let $G$ be a reductive group giving rise to a Shimura datum, with associated Shimura variety $S h_{K}$, for compact open $K \subset G\left(\mathbb{A}_{f}\right)$. For $K^{\prime} \subset K$ normal, there is a natural Galois etale covering

$$
\pi_{K^{\prime}}: S h_{K^{\prime}} \rightarrow S h_{K}
$$

with Galois group $K / K^{\prime}$. We choose a system of $K^{\prime}$ such that $K^{\prime}$ differs from $K$ only in $l$-adic part $K_{l}^{\prime}$; that is, $K / K^{\prime}=K_{l} / K_{l}^{\prime}$, and their $l$-adic parts $K_{l}^{\prime}$ form a basis of $G\left(\mathbb{Q}_{l}\right)$. Let $V$ be an algebraic representation of $G$, then it gives rise to a continuous $l$-adic representation of $G\left(\mathbb{Q}_{l}\right)$, which contains a lattice $\Lambda$ stable by all $K_{l}^{\prime}$, and for $K_{l}^{\prime} \subset K_{l}$, there exists a number $n$ such that the natural action of $K_{l}^{\prime}$ and $K_{l}$ on $\Lambda$ induces a representation of $K_{l} / K_{l}^{\prime}$ on $\Lambda / l^{n} \Lambda$, then we have an etale sheaf

$$
\mathcal{V}_{K^{\prime}}:=\left(\pi_{K^{\prime} *}\left(\mathbb{Z} / l^{n} \mathbb{Z}\right) \otimes_{\mathbb{Z} / l^{n} \mathbb{Z}} \Lambda / l^{n} \Lambda\right)^{K_{l} / K_{l}^{\prime}}
$$


where the action of $K_{l} / K_{l}^{\prime}$ on the first factor is induced by the Galois covering $\pi_{K^{\prime}}$ and the second factor is induced by the representation we have just constructed. These $\mathcal{V}_{K^{\prime}}$ form an inverse system, and we define the associated $l$-adic sheaf by

$$
\mathcal{F} V:=\left({\stackrel{\lim }{K^{\prime}}}_{\mathcal{V}_{K^{\prime}}}\right) \otimes_{\mathbb{Z}_{l}} \overline{\mathbb{Q}_{l}}
$$

This is independent of the choices we have made. Similar to Hecke operators, the partial Frobenius induces natural maps between $\mathcal{F} V$; that is, $\mathcal{F} V \longrightarrow F_{i *} \mathcal{F V}$. The key to it is that the partial Frobenius is compatible with the projections $\pi_{K^{\prime}}$; that is,

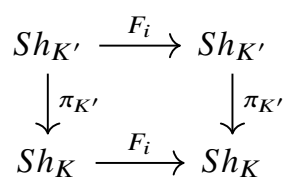

is commutative and equivariant for the Galois group. It is a general heuristic that the partial Frobenius is amplified Hecke operators in characteristic $p$.

Moreover, the isomorphism (21) is compatible with the partial Frobenius. As in Theorem 3.16 (for PEL Shimura varities), the partial Frobenius $F_{i}$ extends to the minimal compactification and preserves both the open $S h_{K} \stackrel{j}{\hookrightarrow} S h_{K}^{\text {min }}$ and the boundary $S h_{K}^{\text {min }} \backslash S h_{K} \stackrel{i}{\hookrightarrow} S h_{K}^{\text {min }}$, therefore inducing the map

$$
i^{*} R j_{*} \overline{\mathbb{Q}_{l}} \stackrel{i^{*} R j_{*}(-)}{\longrightarrow} i^{*} R j_{*} F_{i *} \overline{\mathbb{Q}_{l}}=i^{*} F_{i *} R j_{*} \overline{\mathbb{Q}_{l}} \stackrel{\text { b.c. }}{\longrightarrow} F_{i *} i^{*} R j_{*} \overline{\mathbb{Q}_{l}}
$$

which under the natural isomorphism (21), corresponds to the natural map $\mathcal{F} V \rightarrow F_{i *} \mathcal{F} V$ for $V$ specified in (21).

Remark 4.2. The above naturality can be proved with the same proof as in Section 4.8 of [29], where it is proved for the Hecke operators. The key property underlying the proof is the compatibility of Hecke operators with the toroidal compactifications. The same compatibility result holds for the partial Frobenius, as we will see in the next section.

Now we go back to the special case of Hilbert modular varieties. Applying the above functoriality to the isomorphism (22), we can reduce the computation of $i^{*} R j_{*} \overline{\mathbb{Q}_{l}} \rightarrow F_{i *} i^{*} R j_{*} \overline{\mathbb{Q}_{l}}$ to the computation of $\mathcal{F} V \rightarrow F_{i *} \mathcal{F} V$ for $V$ as in (22).

We make use of the parametrisation (23). For an arbitrary integer $k$, let

$$
\pi_{k}: \operatorname{Isom}_{\mathcal{O}_{F}}\left(\mathfrak{d}_{F}^{-1} / n l^{k} \mathfrak{d}_{F}^{-1}(1), \mathfrak{d}_{F}^{-1} \otimes_{\mathbb{Z}} \mu_{n l^{k}}\right) \rightarrow \operatorname{Isom}_{\mathcal{O}_{F}}\left(\mathfrak{d}_{F}^{-1} / n \mathfrak{D}_{F}^{-1}(1), \mathfrak{d}_{F}^{-1} \otimes_{\mathbb{Z}} \mu_{n}\right)
$$

be the natural map, corresponding to the covering map $\pi_{K^{\prime}}$ as above. Let $\theta \in \operatorname{Isom}_{\mathcal{O}_{F}}\left(\mathfrak{D}_{F}^{-1} / n \mathfrak{D}_{F}^{-1}(1)\right.$, $\left.\mathfrak{D}_{F}^{-1} \otimes_{\mathbb{Z}} \mu_{n}\right)$, and we suppose that $\theta$ lies in the position $(\alpha, \delta, \partial)$ of the decomposition

$$
\mathscr{M}_{\mathbb{F}_{p}}^{*} \backslash \mathscr{M}_{\mathbb{F}_{p}}=\coprod_{\alpha \in \Omega} \coprod_{\partial} \operatorname{Isom}_{\mathcal{O}_{F}}\left(\mathfrak{D}_{F}^{-1} / n \mathfrak{D}_{F}^{-1}(1), \mathfrak{D}_{F}^{-1} \otimes_{\mathbb{Z}} \mu_{n}\right) .
$$

Recall that $F_{i}$ maps $(\alpha, \delta, \partial)$ to $\left(\alpha_{1}, \delta_{1}, \partial_{1}\right)$, where $\alpha_{1}$ is defined by

$$
\xi \alpha=\epsilon_{1} \alpha_{1} \lambda_{1}
$$

with $\xi \in \mathcal{O}_{F}$ such that $v_{\mathfrak{p}_{i}}(\xi)=1$ and $v_{\mathfrak{p}_{j}}(\xi)=0$ for $j \neq i, \alpha_{1} \in \Omega, \epsilon_{1} \in\left(\mathcal{O}_{F} \otimes \mathbb{Z}_{(p)}\right)_{+}^{\times}$and $\lambda_{1} \in\left(\mathcal{O}_{F} \otimes \hat{\mathbb{Z}}^{p}\right)^{\times}$as in decomposition (15). Moreover, $\partial_{1}$ is defined as in Theorem 3.16 (being a union of $\partial^{\prime}$ in Theorem 3.16), and $F_{i}$ maps $\theta$ to $\lambda_{1} \theta$ as in Definition 3.13. The vague description of $\partial_{1}$ here 
suffices for our purpose. In summary,

$$
\left.\left.\theta\right|_{(\alpha, \partial)} \stackrel{F_{i}}{\longrightarrow}\left(\lambda_{1} \theta\right)\right|_{\left(\alpha_{1}, \partial_{1}\right)}
$$

with obvious notations.

We can repeat the above procedure and obtain

$$
\begin{gathered}
\xi \alpha_{1}=\epsilon_{2} \alpha_{2} \lambda_{2} \\
\vdots \\
\xi \alpha_{m}=\epsilon_{m+1} \alpha_{m+1} \lambda_{m+1},
\end{gathered}
$$

where $\alpha_{j} \in \Omega, \epsilon_{j} \in\left(\mathcal{O}_{F} \otimes \mathbb{Z}_{(p)}\right)_{+}^{\times}$and $\lambda_{j} \in\left(\mathcal{O}_{F} \otimes \hat{\mathbb{Z}}^{p}\right)^{\times}$as in decomposition (15). Then

$$
\left.\left.\theta\right|_{(\alpha, \partial)} \stackrel{F_{i}^{m}}{\longrightarrow}\left(\lambda_{1} \cdots \lambda_{m} \theta\right)\right|_{\left(\alpha_{m}, \partial_{m}\right)} .
$$

Because $F_{i}$ permutes the cusps, we know that there is a minimal integer $N$ such that

$$
F_{i}^{N}(\theta)=\theta
$$

Note that this means that $\lambda_{1} \cdots \lambda_{N} \theta=\theta, \alpha_{N}=\alpha$ and $\partial_{N}=\partial$.

We denote by $\widetilde{\mathscr{M}}$ the Hilbert modular variety of principal level $n l^{k}$, then we have a natural commutative diagram map

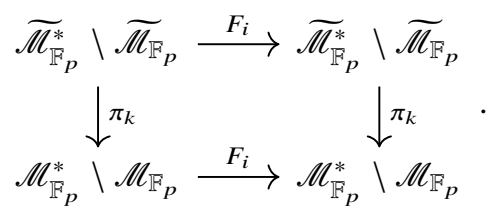

Together with the decomposition

$$
\widetilde{\mathscr{M}_{\mathbb{F}_{p}}^{*}} \backslash \widetilde{\mathscr{M}_{\mathbb{F}_{p}}}=\coprod_{\alpha \in \Omega} \coprod_{\widetilde{\partial}} \operatorname{Isom}_{\mathcal{O}_{F}}\left(\mathfrak{D}_{F}^{-1} / n l^{k} \mathfrak{D}_{F}^{-1}(1), \mathfrak{D}_{F}^{-1} \otimes_{\mathbb{Z}} \mu_{n l^{k}}\right)
$$

we are reduced to the situation

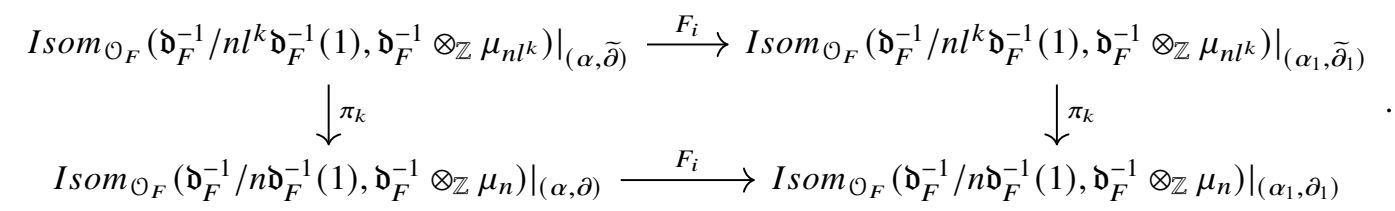

The same description of $F_{i}$ applies to $\widetilde{M}_{\mathbb{F}_{p}}$. In summary,

$$
\left.\left.\widetilde{\theta}\right|_{(\alpha, \widetilde{\partial})} \stackrel{F_{i}}{\longrightarrow}\left(\lambda_{1} \widetilde{\theta}\right)\right|_{\left(\alpha_{1}, \widetilde{\partial}_{1}\right)}
$$

One subtlety here is that there are more than one $\widetilde{\partial}$ lying over $\partial$. However, the cusps they parametrise are canonically isomorphic, and we can choose one $\partial \vec{\partial}$ for each $\partial$.

For simplicity, we assume that $l$ is prime to $n$, then the Galois group for the covering $\pi_{k}$ is $\left(\mathcal{O}_{F} / l^{k} \mathcal{O}_{F}\right)^{\times}$. If we denote by $V$ the 1-dimensional representation $N m_{F / \mathbb{Q}}^{-1}: \operatorname{Res}_{F / \mathbb{Q}} \mathbf{G}_{m} \rightarrow \mathbf{G}_{m}$, then 
its $l$-adic points induces the reduced representation $N m_{F / \mathbb{Q}}^{-1}:\left(\mathcal{O}_{F} / l^{k} \mathcal{O}_{F}\right)^{\times} \longrightarrow\left(\mathbb{Z} / l^{k} \mathbb{Z}\right)^{\times}$, which we denote by $\mathcal{V}_{k}$. We fix a nonzero element $v_{k} \in \mathcal{V}_{k}$ for each $k$, and we assume that they are compatible when $k$ varies. From the description we have just reviewed, we have

$$
\mathcal{F} V=(\underbrace{\lim }_{k}\left(\pi_{k *}\left(\mathbb{Z} / l^{k} \mathbb{Z}\right) \otimes_{\mathbb{Z} / l^{k} \mathbb{Z}} V_{k}\right)^{\left(\mathcal{O}_{F} / l^{k} \mathcal{O}_{F}\right)^{\times}}) \otimes_{\mathbb{Z}_{l}} \overline{\mathbb{Q}_{l}}
$$

For a fixed $k$, if we choose a

$$
\widetilde{\theta} \in \operatorname{Isom}_{\mathcal{O}_{F}}\left(\mathfrak{D}_{F}^{-1} / n l^{k} \mathfrak{D}_{F}^{-1}(1), \mathfrak{D}_{F}^{-1} \otimes_{\mathbb{Z}} \mu_{n l^{k}}\right)
$$

such that $\pi_{k}(\widetilde{\theta})=\theta$, then

$$
\left.\pi_{k *}\left(\mathbb{Z} / l^{k} \mathbb{Z}\right) \otimes_{\mathbb{Z} / l^{k} \mathbb{Z}} \mathcal{V}_{k}\right)\left.^{\left(\mathcal{O}_{F} / l^{k} \mathcal{O}_{F}\right)^{\times}}\right|_{\theta}=\left(\mathbb{Z} /{ }^{k} \mathbb{Z}\right) \cdot \sum_{g \in\left(\mathcal{O}_{F} / l^{k} \mathcal{O}_{F}\right)^{\times}}(g \widetilde{\theta}) \otimes\left(N m_{F / \mathbb{Q}}^{-1}(g) v_{k}\right)
$$

that is, the choice of $v_{k}$ and $\widetilde{\theta}$ gives a basis $\sum_{g \in\left(\mathcal{O}_{F} / l^{k} \mathcal{O}_{F}\right)^{\times}}(g \widetilde{\theta}) \otimes\left(N m_{F / \mathbb{Q}}^{-1}(g) v_{k}\right)$ of $\pi_{k *}\left(\mathbb{Z} / l^{k} \mathbb{Z}\right) \otimes_{\mathbb{Z} / l^{k} \mathbb{Z}}$ $\left.\mathcal{V}_{k}\right)\left.^{\left(\mathcal{O}_{F} / l^{k} \mathcal{O}_{F}\right)^{\times}}\right|_{\theta}$

Now using this explicit description, we can compute the natural morphism $\mathcal{F} V \rightarrow F_{i *} \mathcal{F V}$ (over $\left.\mathscr{M}_{\mathbb{F}_{p}}^{*} \backslash \mathscr{M}_{\mathbb{F}_{p}}\right)$ as follows. It is $\left(\overline{\mathbb{Q}}_{l} \otimes(-)\right)$ the direct limit of the morphism

$$
\left.\left.\sum_{g \in\left(\mathcal{O}_{F} / l^{k} \mathcal{O}_{F}\right)^{\times}}(g \widetilde{\theta}) \otimes\left(N m_{F / \mathbb{Q}}^{-1}(g) v_{k}\right)\right|_{(\alpha, \partial)} \rightarrow \sum_{g \in\left(\mathcal{O}_{F} / l^{k} \mathcal{O}_{F}\right)^{\times}}\left(g \lambda_{1} \widetilde{\theta}\right) \otimes\left(N m_{F / \mathbb{Q}}^{-1}(g) v_{k}\right)\right|_{\left(\alpha_{1}, \partial_{1}\right)} .
$$

For a fixed $\theta$ and the corresponding minimal $N$ as above, we can iterate the process and obtain a basis for the stalk of the sheaf at $F_{i}^{m}\left(\left.\theta\right|_{(\alpha, \partial)}\right)$ for $m<N$. Note that by the choice of $N, F_{i}^{m}\left(\left.\theta\right|_{(\alpha, \partial)}\right)$ are all different for $m<N$. When $m=N$, we have $F_{i}^{N}\left(\left.\theta\right|_{(\alpha, \partial)}\right)=\left.\theta\right|_{(\alpha, \partial)}$, and

$$
\begin{array}{r}
\sum_{g \in\left(\mathcal{O}_{F} / l^{k} \mathcal{O}_{F}\right)^{\times}}(g \widetilde{\theta}) \otimes\left(N m_{F / \mathbb{Q}}^{-1}(g) v_{k}\right) \stackrel{F_{i}^{N}}{\longrightarrow} \sum_{g \in\left(\mathcal{O}_{F} / l^{k} \mathcal{O}_{F}\right)^{\times}}\left(g \lambda_{1} \cdots \lambda_{N} \widetilde{\theta}\right) \otimes\left(N m_{F / \mathbb{Q}}^{-1}(g) v_{k}\right) \\
=N m_{F / \mathbb{Q}}\left(\lambda_{1} \cdots \lambda_{N}\right) \sum_{g \in\left(\mathcal{O}_{F} / l^{k} \mathcal{O}_{F}\right)^{\times}}(g \widetilde{\theta}) \otimes\left(N m_{F / \mathbb{Q}}^{-1}(g) v_{k}\right) .
\end{array}
$$

This tells us that with the basis we have chosen, $F_{i}$ has a block of the form

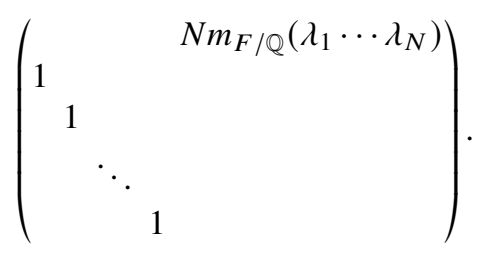

This is a matrix expression of a morphism between free $\mathbb{Z} / l^{k} \mathbb{Z}$-modules. Taking the inverse limit over $k$ and tensor with $\overline{\mathbb{Q}_{l}}$, we have the same matrix (partial expression) for the desired morphism $\mathcal{F} V \rightarrow F_{i *} \mathcal{F} V$. Now from equation (26) and $\alpha_{N}=\alpha$, we have

$$
\xi^{N} \alpha=\left(\epsilon_{1} \cdots \epsilon_{N}\right) \alpha\left(\lambda_{1} \cdots \lambda_{N}\right)
$$


with $\left(\epsilon_{1} \cdots \epsilon_{N}\right) \in\left(\mathcal{O}_{F} \otimes \mathbb{Z}_{(p)}\right)_{+}^{\times}$and $\lambda_{1} \cdots \lambda_{N} \in\left(\mathcal{O}_{F} \otimes \hat{\mathbb{Z}}^{p}\right)^{\times}$. Hence,

$$
\lambda_{1} \cdots \lambda_{N}=\xi^{N}\left(\epsilon_{1} \cdots \epsilon_{N}\right)^{-1}
$$

and

$$
N m_{F / \mathbb{Q}}\left(\lambda_{1} \cdots \lambda_{N}\right)=N m_{F / \mathbb{Q}}\left(c^{N}\right)=p^{N}
$$

It is easy to compute that the characteristic polynomial of the matrix

$$
\left(\begin{array}{lllll} 
& & & & p^{N} \\
1 & & & \\
& 1 & & \\
& & \ddots & \\
& & & 1
\end{array}\right)
$$

is $x^{N}-p^{N}$; hence, the eigenvalues are of the form $p \zeta_{N}^{i}$ with $\zeta_{N}$ a primitive $N$ th root of unity. Therefore, they are Weil numbers with absolute value $p$. Because every block is of the above form, we see that the eigenvalues are all of absolute value $p$. If we base change everything to $\overline{\mathbb{F}}_{p}$, then the above computation computes the eigenvalues of the partial Frobenius $F_{i}$ on $H^{*}\left(\mathscr{M}_{\overline{\mathbb{F}}_{p}}^{*} \backslash \mathscr{M}_{\overline{\mathbb{F}}_{p}}, \mathcal{F} V\right)$, which we see are all of absolute value $p$. Then from ( $l$-adic realisation of) equation (25), we have that $w_{\geq k} w_{\leq k} R^{n} j_{*} \overline{\mathbb{Q}_{l}}$ is a sum of $i_{*} \mathcal{F} V$ if $k>0$; hence, the partial Frobenius acts on

$$
H^{*}\left(\mathscr{M}_{\overline{\mathbb{F}}_{p}}^{*}, w_{\geq k} w_{\leq k} R j_{*} \overline{\mathbb{Q}_{l}}\right)
$$

with eigenvalues of absolute value $p$, if $k>0$.

To summarise, we have proven the following proposition.

Proposition 4.3. The partial Frobenius $F_{i}$ acts on the spectral sequence (18) by Proposition 2.18. More precisely, by Proposition 2.18, $F_{i}$ acts on the special fibre variant of (l-adic realisation of) the spectral sequence (18)

$$
E_{1}^{a, b}=H^{a+b}\left(\mathscr{M}_{\overline{\mathbb{F}}_{p}}^{*}, w_{\geq-a} w_{\leq-a} R j_{*} \overline{\mathbb{Q}_{l}}\right) \Rightarrow H^{a+b}\left(\mathscr{M}_{\overline{\mathbb{F}}_{p}}, \overline{\mathbb{Q}_{l}}\right),
$$

which is (at least up to convergence) isomorphic to the Hodge module realisation of (18) by Theorem 2.16 and choice of $p$. If $a<0, F_{i}$ acts on $E_{1}^{a, b}$ with eigenvalues of absolute value $p$ and hence of partial Frobenius weights $(2, \cdots, 2)$.

On the other hand, the Hodge module realisation of (18) has ${ }^{H} E_{1}^{a, b}=$ $H^{a+b}\left(\mathscr{M}^{*}(\mathbb{C}), w_{\geq-a} w_{\leq-a} R j_{*} \mathbb{C}\right)$, which is a sum of $H^{a+b}\left(\mathscr{M}^{*}(\mathbb{C}) \backslash \mathscr{M}(\mathbb{C}), \mathcal{F} V\right)$ if $a<0$, and hence of plectic Hodge type $(1, \cdots, 1 ; 1, \cdots, 1)$ (sum of $\mathbb{C}(-1)^{\otimes d}$, the $(-d)$ th power of Tate structure). These are of plectic weight $(2, \cdots, 2)$, and the above computation shows that under the comparison, the partial Frobenius weights are the same as the plectic Hodge weights.

Remark 4.4. It is possible to avoid the comparison Theorem 2.16 in the special case of Hilbert modular varieties. We have observed that the spectral sequence (18) induces (shifts of) the weight filtration on the open cohomology. Therefore, the comparison automatically holds. To spell this out, we note that the identification with the weight filtration gives a motivic meaning of the filtration induced by (18), namely, we can find a smooth projective compactification with smooth normal crossing boundary divisors, and the filtration can be expressed in terms of the cohomology of the natural strata. Then the comparison is reduced to the standard comparison between different cohomology theories.

Note that in general the filtration induced by the spectral sequence in Theoerem 2.14 is not the weight filtration. However, in some sense, it detects the nontrivial extensions of the weight filtration. 
We have computed the partial Frobenius on $E_{1}^{p, q}$ for $p<0$ and checked that the partial Frobenius weights are the same as the plectic Hodge weights. It remains to do the same for the remaining $E_{1}^{0, q}=I H^{q}\left(\mathscr{M}_{\overline{\mathbb{F}}_{p}}^{*}, \overline{\mathbb{Q}_{l}}\right)$.

We note that the Hecke algebra decomposes the cohomology into

$$
I H^{*}\left(\mathscr{M}_{\overline{\mathbb{F}}_{p}}^{*}, \overline{\mathbb{Q}_{l}}\right)=I H^{*}\left(\mathscr{M}_{\overline{\mathbb{F}}_{p}}^{*}, \overline{\mathbb{Q}_{l}}\right)_{\text {cusp }} \oplus I H^{*}\left(\mathscr{M}_{\overline{\mathbb{F}}_{p}}^{*}, \overline{\mathbb{Q}_{l}}\right)_{\text {rest }},
$$

where $I H^{*}\left(\mathscr{M}_{\overline{\mathbb{F}}_{p}}^{*}, \overline{\mathbb{Q}_{l}}\right)_{\text {cusp }}$ is the subspace on which the Hecke algebra acts with the same type as some cuspidal automorphic representations. Similarly, $I H^{*}\left(\mathscr{M}_{\overline{\mathbb{F}}_{p}}^{*}, \overline{\mathbb{Q}_{l}}\right)_{\text {rest }}$ is the subspace on which the Hecke algebra acts as a discrete but noncuspidal automorphic representation.

Note that the corresponding representation is cohomological and we can classify them. The cuspidal part corresponds to holomorphic Hilbert modular forms of weight $(2, \cdots, 2)$, and the discrete noncuspidal part corresponds to 1-dimensional representations.

We first compute the cuspidal part. We have

$$
I H^{*}\left(\mathscr{M}_{\overline{\mathbb{F}}_{p}}^{*}, \overline{\mathbb{Q}_{l}}\right)_{\text {cusp }}=\underset{f}{\oplus I} H^{*}\left(\mathscr{M}_{\overline{\mathbb{F}}_{p}}^{*}, \overline{\mathbb{Q}_{l}}\right)_{f}
$$

where $f$ ranges over holomorphic Hilbert modualr forms of weight $(2, \cdots, 2)$; see [12] chapter 3, for example. It is well known from the $(g, K)$-cohomology computations that $I H^{*}\left(\mathscr{M}_{\overline{\mathrm{F}}_{p}}^{*}, \overline{\mathbb{Q}_{l}}\right)_{f}$ is concentrated in degree $d$ and (its complex variant) has plectic Hodge type $((1,0) \oplus(0,1))^{\otimes d}$, hence of plectic weight $(1, \cdots, 1)$. We want to check that the partial Frobenius weights are again of the same weight, namely, the eigenvalues of the partial Frobenius $F_{i}$ on $I H^{*}\left(\mathscr{M}_{\overline{\mathbb{F}}_{p}}^{*}, \overline{\mathbb{Q}_{l}}\right)_{f}$ have absolute value $p^{\frac{1}{2}}$.

Recall that Nekovár proved in [25] that the partial Frobenius satisfies an Eichler-Shimura relation. In the Hilbert modular case, it is

$$
F_{i}^{2}-\left(T_{i} / S_{i}\right) F_{i}+p / S_{i}=0
$$

where $T_{i}, S_{i}$ are standard Hecke operators of the Hecke algebra of $\operatorname{Res}_{F / \mathbb{Q}} G L_{2}$ at $\mathbb{Q}_{p}$; that is,

$$
T_{i}, S_{i} \in H\left(\operatorname{Res}_{F / \mathbb{Q}} G L_{2}\left(\mathbb{Q}_{p}\right) / / \operatorname{Res}_{F / \mathbb{Q}} G L_{2}\left(\mathbb{Z}_{p}\right), \mathbb{Z}\right)=\otimes_{i} H\left(G L_{2}\left(\mathbb{Q}_{p}\right) / / G L_{2}\left(\mathbb{Z}_{p}\right), \mathbb{Z}\right)
$$

indexed by $\left\{\mathfrak{p}_{i}\right\}$; see [25] A6. The upshot is that this shows that the eigenvalues of the partial Frobenius $F_{i}$ on $I H^{*}\left(\mathscr{M}_{\overline{\mathbb{F}}_{p}}^{*}, \overline{\mathbb{Q}}_{l}\right)_{f}$ are the same as the eigenvalues of the (geometric) Frobenius Frob ${\mathfrak{p}_{i}}_{i}$ on the representation $\rho_{f}^{\vee}(-1)$, where $\rho_{f}: \operatorname{Gal}(\overline{\mathbb{Q}} / F) \rightarrow G L_{2}\left(\overline{\mathbb{Q}_{l}}\right)$ is the Galois representation associated to the Hilbert modular form $f$. We know from [3] that the Galois representation $\rho_{f}$ is pure of weight 1 and so is $\rho_{f}^{\vee}(-1)$, proving that the eigenvalues of the partial Frobenius $F_{i}$ on $I H^{*}\left(\mathscr{M}_{\overline{\mathbb{F}}_{p}}^{*}, \overline{\mathbb{Q}_{l}}\right)_{f}$ have absolute value $p^{\frac{1}{2}}$.

Finally, we deal with $I H^{*}\left(\mathscr{M}_{\overline{\mathbb{F}}_{p}}^{*}, \overline{\mathbb{Q}}_{l}\right)_{\text {rest }}$. It is known that it is concentrated in even degrees, and

$$
I H^{2 k}\left(\mathscr{M}_{\overline{\mathbb{F}}_{p}}^{*}, \overline{\mathbb{Q}_{l}}\right)_{\text {rest }}=\bigwedge^{k}\left(I H^{0}\left(\mathscr{M}_{\overline{\mathbb{F}}_{p}}^{*}, \overline{\mathbb{Q}_{l}}\right)_{\text {rest }} \oplus I H^{2}\left(\mathscr{M}_{\mathbb{F}_{p}}^{*}, \overline{\mathbb{Q}_{l}}\right)_{\text {rest }}\right) .
$$

The same holds for the complex variant ([12] chapter 3); thus, it is enough to concentrate on $I H^{2}\left(\mathscr{M}_{\mathbb{F}_{p}}^{*}, \overline{\mathbb{Q}_{l}}\right)_{\text {rest }}$. If we look at a connected component $\mathscr{M}_{\mathbb{\mathbb { F }}_{p}, o}^{*}$ of $\mathscr{M}_{\mathbb{F}_{p}}^{*}$, we have

$$
I H^{2}\left(\mathscr{M}_{\overline{\mathbb{F}}_{p}, o}^{*}, \overline{\mathbb{Q}_{l}}\right)_{\mathrm{rest}}=\underset{i}{\oplus_{\mathbb{Q}}} \cdot c_{1}\left(L_{i}\right)(-1),
$$

where $L_{i}$ is a line bundle on $\mathscr{M}_{\overline{\mathbb{F}}_{p}, o}$ to be defined below, and the equality is interpreted as $c_{1}\left(L_{i}\right)(-1) \in$ 
$H^{2}\left(\mathscr{M}_{\overline{\mathbb{F}}_{p}, o}, \overline{\mathbb{Q}_{l}}\right)$ lying in the image of the natural embedding $I H^{2}\left(\mathscr{M}_{\overline{\mathbb{F}}_{p}, o}^{*}, \overline{\mathbb{Q}_{l}}\right)_{\text {rest }} \hookrightarrow H^{2}\left(\mathscr{M}_{\overline{\mathbb{F}}_{p}, o}, \overline{\mathbb{Q}_{l}}\right)$. Let $p: \mathcal{A} \rightarrow \mathscr{M}_{\mathbb{F}_{p}, o}$ be the universal abelian scheme over $\mathscr{M}_{\mathbb{F}_{p}, o}$, then $\operatorname{Lie}_{\mathcal{A} / \mathscr{M}_{\mathbb{F}}, o}^{\vee}$ is a coherent sheaf of projective $\mathcal{O}_{F} \otimes_{\mathbb{Z}} \mathbb{F}_{p}$-module with rank 1 . By the choice of $p$, we have $\mathcal{O}_{F} \otimes_{\mathbb{Z}} \mathbb{F}_{p}=\prod_{i} \mathbb{F}_{p}$ parametrised by $\left\{\mathfrak{p}_{i}\right\}$; hence,

$$
\operatorname{Lie}_{\mathcal{A} / \mathscr{M}_{\mathbb{F} p}, o}^{\vee}=\underset{i}{\oplus} L_{i}
$$

where $L_{i}:=e_{i} L_{i} e_{\mathcal{A} / \mathscr{M}_{\mathbb{F}}, o}^{\vee}$ and $e_{i}$ is the idempotent of $\prod_{i} \mathbb{F}_{p}$ corresponding to the $i$ th factor. Another way to characterise $L_{i}$ is to note that

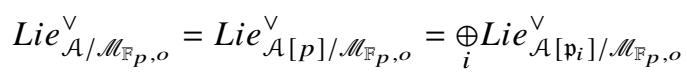

and

$$
L_{i}=\operatorname{Lie}_{\mathcal{A}\left[\mathfrak{p}_{i}\right] / \mathscr{M}_{\mathbb{F} p}, o} \cdot
$$

Now by definition of the partial Frobenius $F_{i}$, we have a Cartesian diagram

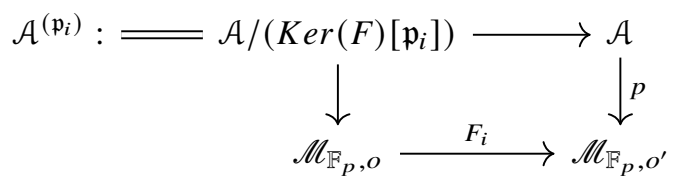

with a possibly different connected component $\mathscr{M}_{\mathbb{F}_{p}, o^{\prime}}$. By abuse of notation, we use the same $\mathcal{A}$ to denote the universal abelian scheme on $\mathscr{M}_{\mathbb{F}_{p}, o^{\prime}}$, and similarly for $L_{i}$. The diagram tells us that

$$
F_{i}^{*}\left(L_{i}\right)=e_{j} \operatorname{Lie}_{\mathcal{A}^{\left(p_{i}\right)} / \mathscr{M}_{\mathbb{F}}, o}=\operatorname{Lie}_{\mathcal{A}^{\left(\mathfrak{p}_{i}\right)}\left[\mathfrak{p}_{j}\right] / \mathscr{M}_{\mathbb{F} p, o}} .
$$

If $j \neq i$, then clearly $\mathcal{A}^{\left(\mathfrak{p}_{i}\right)}\left[\mathfrak{p}_{j}\right]=\mathcal{A}\left[\mathfrak{p}_{j}\right]$; hence,

$$
F_{i}^{*}\left(L_{j}\right)=\operatorname{Lie}_{\mathcal{A}\left[\mathfrak{p}_{j}\right] / \mathscr{M}_{\mathbb{F} p, o}}=L_{j} .
$$

If $j=i$, then $\mathcal{A}^{\left(\mathfrak{p}_{i}\right)}\left[\mathfrak{p}_{i}\right]=\mathcal{A}^{(p)}\left[\mathfrak{p}_{i}\right]$, where $\mathcal{A}^{(p)}:=\mathcal{A} / \operatorname{Ker}(F)$ as usual; hence,

$$
\begin{gathered}
F_{i}^{*}\left(L_{i}\right)=\operatorname{Lie}_{\mathcal{A}(p)}\left[\mathfrak{p}_{i}\right] / \mathscr{M}_{\mathbb{F} p, o}=e_{i} \operatorname{Lie}_{\mathcal{A}(p) / \mathscr{M}_{\mathbb{F} p, o}}=e_{i} \text { Frob }^{*} \operatorname{Lie}_{\mathcal{A} / \mathscr{M}_{\mathbb{F} p, o}} \\
\left.=e_{i} \operatorname{Frob}^{*}\left(\underset{j}{\oplus} L_{j}\right)=e_{i}\left(\underset{j}{\oplus} \operatorname{Frob}^{*} L_{j}\right)=e_{i} \underset{j}{\oplus} L_{j}^{\otimes p}\right)=L_{i}^{\otimes p},
\end{gathered}
$$

where Frob : $\mathscr{M}_{\mathbb{F}_{p}, o} \rightarrow \mathscr{M}_{\mathbb{F}_{p}, o}$ is the absolute Frobenius, and we use that $F r o b^{*} L \cong L^{\otimes p}$ for any line bundle $L$ (by looking at the transition function of $L$ ).

Now we have proved that

$$
F_{i}^{*}\left(c_{1}\left(L_{j}\right)\right)=c_{1}\left(F_{i}^{*} L_{j}\right)=\left\{\begin{array}{ll}
c_{1}\left(L_{j}\right) & j \neq i \\
p c_{1}\left(L_{i}\right) & j=i
\end{array} .\right.
$$

Taking the subtlety of the connected components, we see that

$$
I H^{2}\left(\mathscr{M}_{\overline{\mathbb{F}}_{p}}^{*}, \overline{\mathbb{Q}_{l}}\right)_{\mathrm{rest}}=\underset{i}{\oplus} W_{i},
$$


where $W_{i}$ is the subspace generated by $c_{1}\left(L_{i}\right)(-1)$ on each connected component. Then with the modification introduced by base change to algebraic closure and the Tate twist, $F_{i}$ acts on $W_{i}$ with blocks of the form

$$
\left(\begin{array}{llll} 
& & & \\
p & & & \\
& p & & \\
& & \ddots & \\
& & & p
\end{array}\right)
$$

hence we have eigenvalues $p \zeta$ for $\zeta$ some roots of unity. If $j \neq i, F_{i}$ acts on $W_{j}$ with blocks of the form

$$
\left(\begin{array}{llll}
1 & & & \\
& 1 & & \\
& & \ddots & \\
& & & 1
\end{array}\right)
$$

which have eigenvalues roots of unity. This proves that $W_{i}$ have partial Frobenius weights $(0, \cdots, 0,2,0, \cdots 0)$ with 2 at the $i$ th position.

On the other hand, the same process gives line bundles $L_{i}$ on $\mathscr{M}_{\mathbb{C}}$, where we use that $L i e_{\mathcal{A} / \mathscr{M}_{\mathbb{C}}}$ is a sheaf of projective $\mathcal{O}_{F} \otimes_{\mathbb{Z}} \mathbb{C}=\prod_{i} \mathbb{C}$-modules, which are indexed by Archimedean places of $F$. The $L_{i}$ can be further characterised by its transition functions; that is, its sections correspond to holomorphic Hilbert modular forms of weight $(0, \cdots, 0,2,0, \cdots 0)$ with 2 at the $i$ th position. In the comparison between Betti cohomology and $l$-adic cohomology of the special fibre at $p$, we implicitly fix an isomorphism $\overline{\mathbb{Q}_{p}} \cong \mathbb{C}$, which induces an identification between Archimedean places and $p$-adic places of $F$. We can compare the Betti and 1-adic realizations of the first Chern class of $L_{i}$, and the corresponding $W_{i} \subset I H^{2}\left(\mathscr{M}^{*}(\mathbb{C}), \mathbb{C}\right)$ generated by $c_{1}\left(L_{i}\right)$ is easily seen to be of plectic Hodge type

$$
(0, \cdots, 0,1,0, \cdots 0 ; 0, \cdots, 0,1,0, \cdots 0)
$$

with both $1 \mathrm{~s}$ in the $i$ th position $\left(c_{1}\left(L_{i}\right)\right.$ is represented by $d z_{i} \wedge d \bar{z}_{i}$ with $\left.\left(z_{k}\right)_{k} \in \mathbb{H}^{d}\right)$. Thus, $W_{i}$ have plectic weight $(0, \cdots, 0,2,0, \cdots 0)$ with 2 at the $i$ th position, which is compatible with the partial Frobenius weights.

To summarise, we have proved the following theorem.

Theorem 4.5. The partial Frobenius $F_{i}$ acts on the special fibre variant of (l-adic realisation of) the spectral sequence (18)

$$
E_{1}^{p, q}=H^{p+q}\left(\mathscr{M}_{\overline{\mathbb{F}}_{p}}^{*}, w_{\geq-p} w_{\leq-p} R j_{*} \overline{\mathbb{Q}_{l}}\right) \Rightarrow H^{p+q}\left(\mathscr{M}_{\overline{\mathbb{F}}_{p}}, \overline{\mathbb{Q}_{l}}\right)
$$

by Proposition 2.18, which is (at least up to convergence) isomorphic to the Hodge module realisation of (18) by Theorem 2.16 and choice of $p$. The Hodge module spectral sequence exhibits plectic Hodge structures on the graded pieces of the filtration induecd by (18) through $(\mathfrak{g}, K)$-cohomology, and the partial Frobenius weights are compatible with the exhibited plectic Hodge weights on each graded piece.

Corollary 4.6. (Plectic weight filtration) There is a natural increasing $\mathbb{Z}^{d}$-filtration $W_{\underline{a}}$ (defined over C) on $H^{*}(\mathscr{M}(\mathbb{C}), \mathbb{C})$ with $\underline{a}=\left(a_{1}, \cdots, a_{d}\right) \in \mathbb{Z}^{d}$, defined by

$$
W_{\underline{a}}=\bigoplus_{\substack{\left|\beta_{i}\right|=p^{\frac{k_{i}}{2}} \\ k_{i} \leq a_{i}}} V_{\left(\beta_{1}, \cdots, \beta_{d}\right)},
$$


where $V_{\left(\beta_{1}, \cdots, \beta_{d}\right)}$ is the generalised eigenspace of $F_{i}$ with eigenvalue $\beta_{i}$ for all $i$. The action of $F_{i}$ on $H^{*}(\mathscr{M}(\mathbb{C}), \mathbb{C})$ is through the natural comparison isomorphism $H^{*}(\mathscr{M}(\mathbb{C}), \mathbb{C}) \cong l_{*} H^{*}\left(\mathscr{M}_{\overline{\mathbb{F}}_{p}}, \overline{\mathbb{Q}}_{l}\right)$ for some fixed isomorphism $l: \overline{\mathbb{Q}_{l}} \cong \mathbb{C}$.

The filtration is plectic in the sense that there is a natural plectic Hodge structure on $G r_{\underline{a}}^{W}$ with plectic weight $\underline{a}$.

Remark 4.7. We have seen that the graded pieces of the constructed plectic weight filtration are motivic and so are independent of the choice of $p$. However, the filtration might still depend on $p$ a priori. We leave the proof of independence of $p$ to future work.

\section{Toroidal compactifications and the partial Frobenius}

\subsection{Polarised degeneration data}

We begin by recalling the degeneration data of abelian schemes introduced by Faltings-Chai and refined by Kaiwen Lan. It is (almost) a collection of linear algebra objects that characterises the degeneration of abelian varieties into semi-abelian varieties. It is relatively straightforward to find the parametrisation space of the degeneration data, which constitutes the base of a universal degenerating abelian scheme. These are used to glue with the PEL Shimura varieties to form toroidal compactifications. We follow the notations of [18] closely; see also [16] for a minimal summary of definitions.

Let $R$ be a Noetherian normal domain complete with respect to an ideal $I$, with $\sqrt{I}=I$. Let $S:=\operatorname{Spec}(R), K:=\operatorname{Frac}(R), \eta:=\operatorname{Spec}(K), S_{0}:=\operatorname{Spec}(R / I)$ and $S_{\text {for }}:=\operatorname{Spf}(R, I)$.

\subsubsection{Definitions and the theorem}

Definition 5.1. The category $\operatorname{DEG}_{\text {pol }}(R, I)$ has objects $\left(G, \lambda_{\eta}\right)$, where (1) $G$ is an semi-abelian scheme over $S$; that is, a commutative group scheme over $S$ with geometric fibre extensions of abelian varieties by torus, such that the generic fibre $G_{\eta}$ is an abelian variety and such that $G_{0}:=G \times_{S} S_{0}$ is globally an extension

$$
0 \longrightarrow T_{0} \longrightarrow G_{0} \longrightarrow A_{0} \longrightarrow 0
$$

where $T_{0}$ is an isotrivial torus over $S_{0}$; that is, $T_{0}$ becomes split over a finite étale cover of $S_{0}$, and $A_{0}$ is an abelian scheme over $S_{0}$.

(2) $\lambda_{\eta}: G_{\eta} \rightarrow G_{\eta}^{\vee}$ is a polarisation of $G_{\eta}$.

The morphisms in the category are isomorphisms of group schemes over $S$ that respect the polarisations on the generic fibres.

Elements of $\operatorname{DEG}_{\text {pol }}(R, I)$ are called degenerating abelian schemes. We will see that they are equivalent to certain data that are more linear algebraic in nature, called degeneration data, to be defined as follows.

Definition 5.2. The category of degeneration data $\mathrm{DD}_{\mathrm{pol}}(R, I)$ has objects

$$
\left(A, \lambda_{A}, \underline{X}, \underline{Y}, \phi, c, c^{\vee}, \tau\right)
$$

where

(1) $A$ is an abelian scheme over $S$, and $\lambda_{A}: A \rightarrow A^{\vee}$ is a polarisation.

(2) $\underline{X}$ and $\underline{Y}$ are étale sheaves of free commutative groups of the same rank, which can be viewed as étale group schemes over $S$, and $\phi: \underline{Y} \hookrightarrow \underline{X}$ is an injective homomorphism with finite cokernel. 
(3) $c$ and $c^{\vee}$ are homomorphisms

$$
\begin{aligned}
& c: \underline{X} \longrightarrow A^{\vee} \\
& c^{\vee}: \underline{Y} \longrightarrow A
\end{aligned}
$$

such that

$$
\lambda_{A} \circ c^{\vee}=c \circ \phi
$$

(4) $\tau$ is a trivialisation

$$
\tau: \mathbf{1}_{\underline{Y} \times S} \underline{X}, \eta \stackrel{\sim}{\longrightarrow}\left(c^{\vee} \times c\right)^{*} \mathcal{P}_{A, \eta}^{\otimes-1}
$$

of the biextension $\left(c^{\vee} \times c\right)^{*} \mathcal{P}_{A, \eta}^{\otimes-1}$ over the étale group scheme $\left(\underline{Y} \times{ }_{S} \underline{X}\right)_{\eta}$ such that $\left(I d_{Y} \times \phi\right)^{*} \tau$ is symmetric, where $\mathcal{P}_{A}$ is the Poincare line bundle on $A \times_{S} A^{\vee}$, and $\mathbf{1}_{\underline{Y} \times} \times_{S} \underline{X}$ is the structure sheaf of $\underline{Y} \times{ }_{S} \underline{X}$. See [18] Definition 3.2.1.1 for the precise definition of biextension; $\tau$ being a trivialisation of biextensions essentially means that $\tau$ is bilinear in a (the only) reasonable sense, and symmetric means the bilinear form is symmetric.

Moreover, $\tau$ is required to satisfy a positivity condition as follows. Taking a finite étale base change of $S$ if necessary, we assume that $\underline{X}$ and $\underline{Y}$ are constant with values $X$ and $Y$. For each $y \in Y$, the isomorphism

$$
\tau(y, \phi(y)): \mathcal{O}_{S, \eta} \stackrel{\sim}{\longrightarrow}\left(c^{\vee}(y) \times c \circ \phi(y)\right)^{*} \mathcal{P}_{A, \eta}^{\otimes-1}
$$

over the generic fibre extends to a section

$$
\tau(y, \phi(y)): \mathcal{O}_{S} \longrightarrow\left(c^{\vee}(y) \times c \circ \phi(y)\right)^{*} \mathcal{P}_{A}^{\otimes-1}
$$

over $S$, which we still denote by $\tau(y, \phi(y))$. Moreover, for each $y \neq 0$, the induced morphism

$$
\left(c^{\vee}(y) \times c \circ \phi(y)\right)^{*} \mathcal{P}_{A} \longrightarrow \mathcal{O}_{S}
$$

factors through $\underline{I}$, where $\underline{I}$ is the subsheaf of $\mathcal{O}_{S}$ corresponding to the ideal $I \subset R$.

The morphims in the category are defined to be isomorphisms (of $A, \underline{X}$ and $\underline{Y}$ ) over $S$ respecting all of the structures.

Now we can state the first key result.

Theorem 5.3 (Faltings-Chai). There is a functor

$$
F_{p o l}(R, I): D E G_{p o l}(R, I) \longrightarrow D D_{p o l}(R, I)
$$

that induces an equivalence of categories.

Remark 5.4. The inverse functor $\mathrm{DD}_{\text {pol }}(R, I) \longrightarrow \mathrm{DEG}_{\mathrm{pol}}(R, I)$ is called the Mumford quotient construction. We will not describe that in detail.

Remark 5.5. There are a few variants of the categories $\mathrm{DEG}_{\mathrm{pol}}(R, I)$ and $\mathrm{DD}_{\mathrm{pol}}(R, I)$. For example, we can forget about the polarisation $\lambda_{\eta}$, or we can rigidify the situation by replacing the polarisation by an ample line bundle. The equivalence of categories as in the theorem extends to these variants. This explains why we include the lower index pol in the notations. 


\subsubsection{Motivations}

Now we explain the construction of $F_{\mathrm{pol}}$. Essentially, it is to associate linear algebra data to degenerating abelian varieties that also characterise it, and a basic model for this kind of construction is to write a complex abelian variety as $\mathbb{C}^{n} / \Gamma$. However, this is a highly transcendental construction, and it is not obvious how to proceed in our algebraic setting.

The basic idea is to use the Fourier coefficients of theta functions to detect the periods of abelian varieties. More precisely, recall that an abelian variety $A$ over $\mathbb{C}$ has the universal covering $\mathbb{C}^{n}$, and it can be written as $A=\mathbb{C}^{n} / \Gamma$ for some period lattice $\Gamma \subset \mathbb{C}^{n}$. A choice of an ample line bundle $L$ on $A$ gives a positive definite Hermitian form on $\mathbb{C}^{n}$ whose imaginary part $E$ takes integer values on $\Gamma$ and a map $\alpha: \Gamma \rightarrow \mathbb{C}^{\times}$such that $\alpha(x+y)=\alpha(x) \alpha(y) \exp (\pi i E(x, y))$. Then the theta functions are sections of $L$, and an element $s \in \Gamma(A, L)$ is equivalent to a holomorphic function $f: \mathbb{C}^{n} \rightarrow \mathbb{C}$ such that

$$
f(z+\gamma)=f(z) \alpha(\gamma) \exp \left(\frac{1}{2} \pi H(\gamma, \gamma)+\pi H(\gamma, z)\right)
$$

for $z \in \mathbb{C}^{n}, \gamma \in \Gamma$.

Now we can find a rank $n$ sublattice $U \subset \Gamma$ isotropic with respect to $E$, such that

$$
f(z)=\exp (l(z)+B(z, z)) \sum_{\chi \in H o m(U, \mathbb{Z})} c_{\chi} \exp (2 \pi i \chi(z))
$$

for some linear form $l: \mathbb{C}^{n} \rightarrow \mathbb{C}$ and complex bilinear form $B: \mathbb{C}^{n} \times \mathbb{C}^{n} \rightarrow \mathbb{C}$ that depends only on $L$ and is independent of the section $s$; see the first chapter of [22] for details. Hence, $f$ is essentially a function on $\mathbb{C}^{n} / U \stackrel{\text { exp }}{\cong} \mathbb{C}^{\times, n}$. Note that $\operatorname{Hom}(U, \mathbb{Z})$ can be identified with the character group $X:=X\left(\mathbb{C}^{\times, n}\right)$ of the algebraic torus $\mathbb{C}^{\times, n}$, and if we write $q:=\exp (2 \pi i z) \in \mathbb{C}^{n, \times}$, then $\exp (2 \pi i \chi(z))=\chi(q)$ under the above identification. Now the essential part of $f$ has a Fourier expansion

$$
\sum_{\chi \in X} c_{\chi} \chi(q)
$$

for $q \in \mathbb{C}^{\times, n}$. This expression has a potential to be algebraic. The universal cover $\mathbb{C}^{n}$ of $A$ is very transcendental, but it seems that the intermediate quotient $\mathbb{C}^{n} / U \cong \mathbb{C}^{\times, n}$ subsumes all of the transcendental part through the exponential map, and the factorisation $\mathbb{C}^{\times, n} \rightarrow A$ is 'algebraic' in nature. Moreover, because the theta functions define a projective embedding of A (assume that $L$ is very ample), they determine $A$ completely, and in particular the multiplicative periods $Y:=\Gamma / U \subset \mathbb{C}^{\times, n}$. Further, the theta functions are determined by the Fourier coefficients $c_{\chi}$ and hence in principle we can read off the multiplicative periods from $c_{\chi}$.

We can make more explicit the procedure to detect the multiplicative periods from $c_{\chi}$. Note that the functional equation (27) gives the relation (for $\gamma \in \Gamma$ )

$$
c_{\chi}=\alpha(\gamma) \cdot \exp (-l(\gamma)) \cdot \exp (-2 \pi i \chi(\gamma)) \cdot c_{\chi+\phi(\gamma)},
$$

where $\phi: Y \rightarrow X$ is the homomorphism determined by the polarisation $E$, namely, for $y \in \Gamma$ and $x \in U, E(y, x)=\phi(y)(x)$ under the identification $X \cong \operatorname{Hom}(U, \mathbb{Z})(U$ is isotropic with respect to $E$, so it descends to a map on $Y=\Gamma / U)$. Rewriting it in our new multiplicative notation, we have

$$
c_{\chi+\phi(\gamma)}=\chi(l(\gamma)) a(\gamma) c_{\chi}
$$

where $\imath: Y \hookrightarrow \mathbb{C}^{n, \times}$ is the inclusion of the multiplicative periods, and $a: Y \rightarrow \mathbb{C}^{\times}$is a function depending on the line bundle $L$. The desired multiplicative periods are then manifested through the ratio between $c_{\chi}$ and $c_{\chi+\phi(y)}$.

Further, we note that we can give a more direct characterisation of the multiplicative periods $Y$, which is useful when we algebraize the above procedure. Recall that $\operatorname{Hom}(Y, \mathbb{Z})$ is canonically the 
multiplicative periods of the dual abelian variety $A^{\vee}$, so $Y$ is naturally the character group of the multiplicative periods of $A^{\vee}$, which is identified with the character group of the associated algebraic torus of $A^{\vee}$.

To summarise, for $\chi \in X$, the linear maps

$$
c_{\chi}: \Gamma(A, L) \rightarrow \mathbb{C}
$$

defined by the Fourier coefficients detect the multiplicative periods $Y \subset \mathbb{C}^{\times, n}$ of $A$, where $Y$ can be naturally identified with the character group of the algebraic torus associated to the dual abelian variety $A^{\vee}$. More explicitly, the relations

$$
c_{\chi+\phi(\gamma)}=b(\gamma, \chi) a(\gamma) c_{\chi}
$$

characterise a bilinear pairing

$$
b(\cdot, \cdot): Y \times X \rightarrow \mathbb{C}^{\times}
$$

such that $b(\cdot, \phi(\cdot))$ is symmetric, and the multiplicative periods $\imath: Y \hookrightarrow \mathbb{C}^{n, \times}$ are determined by the pairing through $b(\chi, \gamma)=\chi(l(\gamma))$. This is the principle that we aim to algebraize and considerably generalise.

\subsubsection{Equivalent formulation of polarised degeneration data}

Before giving the detailed construction of $F_{\text {pol }}(R, I)$, we first explain the meaning of the tuple in the degeneration data.

First, the étale sheaf $\underline{X}$ and $\underline{Y}$ can be viewed as the character groups of torus $T$ and $T^{\vee}$ over $S$, and the homomorphisms $c$ and $c^{\vee}$ are equivalent to extensions

$$
\begin{gathered}
0 \longrightarrow T \longrightarrow G^{\natural} \longrightarrow A \longrightarrow 0 \\
0 \longrightarrow T^{\vee} \longrightarrow G^{\vee, \natural} \longrightarrow A^{\vee} \longrightarrow 0
\end{gathered}
$$

of commutative group schemes over $S$. Passing to a finite étale cover of $S$ if necessary, we can assume that $T$ is split; hence, $\underline{X}$ is constant with value $X$. We view $G^{\natural}$ as a $T$-torsor over $A$, then because $G^{\natural}$ is relative affine over $A$, we have

$$
G^{\natural} \cong \underline{\operatorname{Spec}}_{\mathscr{O}_{A}}\left(\mathcal{O}_{G^{\natural}}\right) \cong \underline{\operatorname{Spec}}_{\mathscr{O}_{A}}\left(\underset{\chi \in X}{\oplus} \mathcal{O}_{\chi}\right),
$$

where $\mathcal{O}_{\chi}:=c(\chi) \in \operatorname{Pic}^{0}(A / S)$ is the eigensheaf of $\mathcal{O}_{G^{\natural}}$ with weight $\chi$ under the action of $T$. Equivalently, $\mathcal{O}_{\chi}$ is the $\mathbf{G}_{m}$-torsor (viewed as a line bundle) $G^{\natural} \times^{T, \chi} \mathbf{G}_{m}$; that is, the pushout of $0 \rightarrow T \rightarrow G^{\natural} \rightarrow A \rightarrow 0$ along $\chi: T \rightarrow \mathbf{G}_{m}$. This explains the identification of $c$ with the first extension, and similarly for $c^{\vee}$.

Note that $c$ being a group homomorphism equips $\underset{\chi \in X}{\oplus} \mathcal{O}_{\chi}$ with an $\mathcal{O}_{A}$-algebra structure. Further, the $T$-torsor $G^{\natural}$ being a group scheme is equivalent to $c$ taking values in $\operatorname{Pic}^{0}(A)$, which is a consequence of the characterising property $m_{A}^{*} \mathscr{L} \cong p r_{1}^{*} \mathscr{L} \otimes p r_{2}^{*} \mathscr{L}$ of $\mathscr{L} \in P i c^{0}(A / S)$.

Next, the homomorphisms $\phi$ and $\lambda_{A}$ such that

$$
\lambda_{A} \circ c^{\vee}=c \circ \phi
$$

are equivalent to a homomorphism

$$
\lambda: G^{\natural} \longrightarrow G^{\natural, \vee}
$$


of group schemes over $S$ that induces a polarisation $\lambda_{A}$ on $A$. Note that a homomorphism $\lambda$ induces a homomorphism of the extensions

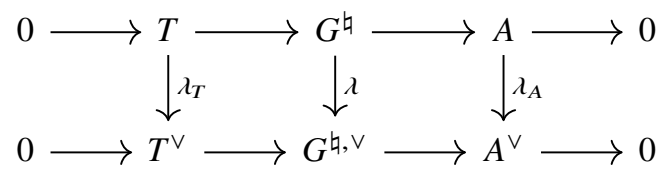

because there is no nontrivial homomorphism from a torus to an abelian variety. Then $\lambda_{A}$ is the induced map on $A$, and $\phi$ is the map on characters induced by $\lambda_{T}$. The relation $\lambda_{A} \circ c^{\vee}=c \circ \phi$ is forced by (and equivalent to) the above commutative diagram.

Lastly and most important, the trivialisation

$$
\tau: \mathbf{1}_{\underline{Y} \times S} \underline{X}, \eta \stackrel{\sim}{\longrightarrow}\left(c^{\vee} \times c\right)^{*} \mathcal{P}_{A, \eta}^{\otimes-1}
$$

of the biextension $\left(c^{\vee} \times c\right)_{A, \eta}^{*} \mathcal{P}_{A-1}^{\otimes-1}$ is equivalent to a group homomorphism

$$
l: \underline{Y}_{\eta} \longrightarrow G_{\eta}^{\natural}
$$

that lifts $c^{\vee}$ over the generic fibre; that is, $c_{\eta}^{\vee}$ factorises as

$$
c_{\eta}^{\vee}: \underline{Y}_{\eta} \stackrel{\imath}{\longrightarrow} G_{\eta}^{\natural} \longrightarrow A_{\eta} .
$$

Again, we can assume that both $\underline{X}$ and $\underline{Y}$ are constant with values $X$ and $Y$, and the general case is by étale descent. Then $\tau$ is a collection of sections $\{\tau(y, \chi)\}_{y \in Y, \chi \in X}$ of the line bundles $\mathcal{P}_{A}\left(c^{\vee}(y), c(\chi)\right)_{\eta}^{\otimes-1}$ on the generic fibre of $S$ for each $y \in Y, \chi \in X$, satisfying bimultiplicative conditions from the biextension structures. Now

$$
\mathcal{P}_{A}\left(c^{\vee}(y), c(\chi)\right)_{\eta}^{\otimes-1}=\left(c^{\vee}(y)^{*} \circ\left(i d_{A} \times c(\chi)\right)^{*} \mathcal{P}_{A}^{\otimes-1}\right)_{\eta}=\left(c^{\vee}(y)^{*} \mathcal{O}_{\chi}^{\otimes-1}\right)_{\eta}
$$

by the definition of $\mathcal{O}_{\chi}$; hence, $\tau(y, \chi)$ is a morphism

$$
\tau(y, \chi): \mathcal{O}_{\chi}\left(c^{\vee}(y)\right)_{\eta} \longrightarrow \mathcal{O}_{S, \eta} .
$$

Together with (28), we have

$$
c^{\vee}(y)^{*} \mathcal{O}_{G^{\natural}, \eta}=c^{\vee}(y)^{*}\left(\underset{\chi \in X}{\oplus} \mathcal{O}_{\chi}\right)^{\sum_{\chi} \tau(y, \chi)} \longrightarrow \mathcal{O}_{S, \eta},
$$

which is a morphism of $\mathcal{O}_{S, \eta}$-algebras by the bimultiplicativity of $\tau$ (more precisely, being an algebra morphism is equivalent to the multiplicativity of the second variable of $\tau$ ). Because $G^{\natural} \cong$ Spec $_{\mathscr{O}_{A}}\left(\mathcal{O}_{G^{\natural}}\right)$ is relative affine over $A$, the algebra morphism is the same as a morphism of $\iota(y): \eta \rightarrow G_{\eta}^{\natural}$ of schemes over $A$; that is,

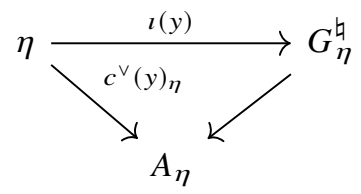

Taking all $y \in Y$ together, we obtain the desired morphism

$$
\iota: Y_{\eta} \rightarrow G_{\eta}^{\natural}
$$


of schemes over $A_{\eta}$. It can be shown that $l$ being a group scheme homomorphism is equivalent to the multiplicativity of the first variable of $\tau$.

In summary, the degeneration data are essentially a commutative group scheme $G^{\natural}$ being an extension of an abelian scheme by a torus over $S$, a period morphism $\imath: Y_{\eta} \rightarrow G_{\eta}^{\natural}$ over the generic fibre and some data specifying the polarisation. We view $G^{\natural}$ as a 'universal cover', and $l$ as the period lattice, parallel to the classical complex case $Y \subset \mathbb{C}^{\times, n}$. Recall that in the definition of degeneration data, $\tau$ has to satisfy the symmetry and positivity condition, which after translation to the setting $\imath: Y_{\eta} \rightarrow G_{\eta}^{\natural}$ is the analogue of the positivity and antisymmetry of the polarisation form $E$ in the classical setting.

Remark 5.6. In the classical complex setting, the existence of $E$ controls the position of the period lattice, and the positivity is the key (equivalent) to finding enough theta functions with respect to the period lattice to embed the quotient complex torus into a projective space. A similar role is played by the conditions on $\tau$. Indeed, given $\imath: Y_{\eta} \rightarrow G_{\eta}^{\natural}$ together with polarisation data, to construct the quotient ' $G$ घ $/ Y_{\eta}$ ' is a highly nontrivial procedure called Mumford construction as mentioned in Remark 5.4. The positivity condition of $\tau$ is a key ingredient in the construction, and the underlying reason seems still to be that the positivity ensures enough theta functions to define a projective embedding.

\subsubsection{The construction of $F_{\text {pol }}$}

Now we can explain the construction of $F_{\text {pol }}$ in the theorem. The first step is to functorially associate a 'universal cover' of $G$ over $S$, and this will be called the Raynaud extension.

We take the formal completion $G_{\text {for }}$ of $G$ along the ideal $I$, which is a formal scheme over $S_{\text {for }}:=$ $\operatorname{Spf}(R, I)$. Because the special fibre $G_{0}:=G \times_{S} S_{0}$ is an extension of an abelian scheme by a torus and torus can be uniquely lifted infinitesimally, we see that $G_{\text {for }}$ is an extension

$$
0 \rightarrow T_{\text {for }} \rightarrow G_{\text {for }} \rightarrow A_{\text {for }} \rightarrow 0
$$

where $T_{\text {for }}$ is a formal torus and $A_{\text {for }}$ is a formal abelian variety. There is an ample cubical (see [18] Definition 3.2.2.7 for definition) invertible sheaf on $G$ whose formal completion descends to an ample sheaf on $A_{\text {for }}$, and Grothendieck existence theorem implies that $A_{\text {for }}$ is algebrizable; that is, $A_{\text {for }}$ is the formal completion of an abelian scheme $A$ over $S$. Note that the existence of an ample invertible sheaf on $G$ is a difficult theorem of Grothendieck, where the key ingredient is that the base $S$ is normal. Now we know that $T_{\text {for }}$ is also algebrizable, whose algebrization we denote by $T$. Then the morphism $X\left(T_{\text {for }}\right) \rightarrow A_{\text {for }}^{\vee}$ corresponding to the extension $G_{\text {for }}$ also algebraizes to a unique morphism $X(T) \rightarrow A^{\vee}$ because the formal completion of proper schemes is a fully faithful functor. The morphism corresponds to the Raynaud extension

$$
0 \rightarrow T \rightarrow G^{\natural} \rightarrow A \rightarrow 0
$$

over $S$.

Now we look at the dual semi-abelian schemes. Because the generic fibre $G_{\eta}$ is an abelian variety, the dual abelian variety $G_{\eta}^{\vee}$ is well defined, and the problem is whether we can extend it naturally to a semi-abelian scheme over $S$. The hard fact is that the closure in $G$ of the finite group scheme $\operatorname{Ker}\left(\lambda_{\eta}\right) \subset G_{\eta}$ is a quasi-finite flat group scheme $\overline{\operatorname{Ker}\left(\lambda_{\eta}\right)}$ over $S$, and the quotient $G / \overline{\operatorname{Ker}\left(\lambda_{\eta}\right)}$ is the desired extension of $G_{\eta}^{\vee}$, which we denote by $G^{\vee}$. The semi-abelian extension to $S$ is unique, so $G^{\vee}$ is uniquely defined. Moreover, the polarisation $\lambda_{\eta}: G_{\eta} \rightarrow G_{\eta}^{\vee}$ extends to a homomorphism

$$
\lambda_{S}: G \longrightarrow G^{\vee}
$$

over $S$.

We can apply the previous argument to $G^{\vee}$ and obtain the Raynaud extension

$$
0 \rightarrow T^{\vee} \rightarrow G^{\vee, \natural} \rightarrow A^{\vee} \rightarrow 0
$$


It can be shown that the abelian part of $G^{\vee, \natural}$ is naturally identified with the dual abelian variety of $A$, explaining the notation. The morphism $\lambda_{S}$ induces the morphisms

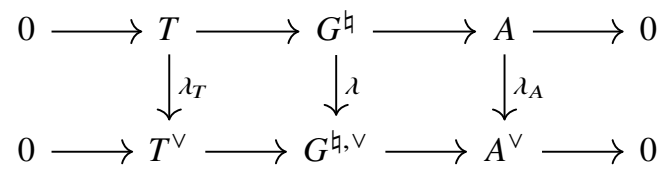

where we can show that $\lambda_{A}$ is a polarisation. By what we have observed, these objects are equivalent to

$$
\left(A, \lambda_{A}, \underline{X}, \underline{Y}, \phi, c, c^{\vee}\right)
$$

in the degeneration data. Hence, we have constructed the first seven objects in $F_{\mathrm{pol}}\left(\left(G, \lambda_{\eta}\right)\right)$.

It remains to construct $\tau$ out of $\left(G, \lambda_{\eta}\right)$. We have seen that $\tau$ essentially corresponds to the periods $\iota: Y_{\eta} \hookrightarrow G_{\eta}^{\natural}$ in the universal cover $G_{\eta}^{\natural}$, and $G=G^{\natural} / Y_{\eta}$ as in the classical case. In particular, as intuition suggests, $\tau$ is determined by $G$ and is independent of the polarisation or ample invertible sheaves used in the construction. Our strategy is to use theta functions to extract the periods, as explained in the motivation part. Indeed, $\tau$ is essentially the analogue of the bilinear form $b(\cdot, \cdot)$ that appears in the functional equations of Fourier coefficients of theta functions in the complex case.

Now we begin to construct $\tau$, following the strategy described in the classical case. Without loss of generality, we assume that $\underline{X}$ and $\underline{Y}$ are constant with values $X$ and $Y$. We choose a cubical ample invertible sheaf $\mathcal{L}$ on $G$, and we can show that its formal completion extends to a cubical ample line bundle $\mathcal{L}^{\natural}$ on $G^{\natural}$. We introduce notations for the maps in the extension by the diagram

$$
0 \rightarrow T \stackrel{i}{\rightarrow} G^{\natural} \stackrel{\pi}{\rightarrow} A \rightarrow 0
$$

and we can choose a cubical trivialisation $i^{*} \mathcal{L}^{\natural} \cong \mathcal{O}_{T}$, which forces $\mathcal{L}^{\natural}$ to descend to an ample invertible sheaf $\mathcal{M}$ on $A$; that is, $\pi^{*} \mathcal{M} \cong \mathcal{L}^{\natural}$. Further, we assume that $\mathcal{L}_{\eta}$ induces the polarisation $\lambda_{\eta}$ on $G_{\eta}$. We can achieve this by possibly replacing $\lambda_{\eta}$ with $\lambda_{\mathcal{L}_{\eta}}$, so the construction of $\tau$ will not depend on the choice of $\lambda_{\eta}$ or $\mathcal{L}$.

We know that $G^{\natural} \cong \operatorname{Spec}_{\mathscr{O}_{A}}\left(\underset{\chi \in X}{\oplus} \mathcal{O}_{\chi}\right)$ as in (28), which implies that

$$
\pi_{*} \mathcal{L}^{\natural} \cong \underset{\chi \in X}{\oplus} \mathcal{M}_{\chi}
$$

where $\mathcal{M}_{\chi}:=\mathcal{M} \otimes_{\mathcal{O}_{A}} \mathcal{O}_{\chi}$. Then by the relative affineness of $G^{\natural}$,

$$
\Gamma\left(G^{\natural}, \mathcal{L}^{\natural}\right)=\Gamma\left(A, \pi_{*} \mathcal{L}^{\natural}\right)=\underset{\chi \in X}{\oplus} \Gamma\left(A, \mathcal{M}_{\chi}\right) .
$$

This is also true if we base change to $S_{i}:=\operatorname{Spec}\left(R / I^{i}\right)$, which forms a compatible system; hence,

$$
\Gamma\left(G_{\text {for }}^{\natural}, \mathcal{L}_{\text {for }}^{\natural}\right) \cong \hat{\oplus}_{\chi \in X} \Gamma\left(A, \mathcal{M}_{\chi}\right)
$$

where the completion is with respect to the $I$-adic topology. Now by the definition of the Raynaud extension, we have that $G$ and $G^{\natural}$ have the same formal completion along $I$; that is, $G_{\text {for }}^{\natural} \cong G_{\text {for }}$. The canonical pullback map $\Gamma(G, \mathcal{L}) \rightarrow \Gamma\left(G_{\text {for }}, \mathcal{L}_{\text {for }}\right)$ becomes

$$
\Gamma(G, \mathcal{L}) \rightarrow \Gamma\left(G_{\text {for }}, \mathcal{L}_{\text {for }}\right) \cong \Gamma\left(G_{\text {for }}^{\natural}, \mathcal{L}_{\text {for }}^{\natural}\right) \cong \underset{\chi \in X}{\hat{\oplus}} \Gamma\left(A, \mathcal{M}_{\chi}\right),
$$


and projecting to the $\chi$ th component we obtain

$$
\Gamma(G, \mathcal{L}) \longrightarrow \Gamma\left(A, \mathcal{M}_{\chi}\right)
$$

Tensoring both sides with $K:=\operatorname{Frac}(R)$, we obtain

$$
\sigma_{\chi}: \Gamma\left(G_{\eta}, \mathcal{L}_{\eta}\right) \longrightarrow \Gamma\left(A_{\eta}, \mathcal{M}_{\chi, \eta}\right)
$$

by flat base change, which are the Fourier coefficients of theta functions with respect to $\mathcal{L}$.

Now as in the classical case, we aim to find the functional equation of $\sigma_{\chi}$ and read off the soughtafter $\tau$ from it. Let $y \in Y$ and $T_{c^{\vee}(y)}: A \rightarrow A$ the translation by the point $c^{\vee}(y)$, then the equation $\lambda_{A} \circ c^{\vee}=c \circ \phi$ applied to $y$ translates into an isomorphism

$$
T_{c^{\vee}(y)}^{*} \mathcal{M}_{\chi} \cong \mathcal{M}_{\chi+\phi(y)} \otimes_{R} \mathcal{M}_{\chi}\left(c^{\vee}(y)\right)
$$

(using rigidified line bundles to represent elements of $A^{\vee}$, and elements of $A^{\vee}$ are characterised by the identity $T_{x}^{*} L \cong L$ ). This provides us with the natural map

$$
T_{c^{\vee}(y)}^{*} \circ \sigma_{\chi}: \Gamma\left(G_{\eta}, \mathcal{L}_{\eta}\right) \rightarrow \Gamma\left(A_{\eta}, T_{c^{\vee}(y)}^{*} \mathcal{M}_{\chi, \eta}\right) \cong \Gamma\left(A_{\eta}, \mathcal{M}_{\chi+\phi(y), \eta}\right) \otimes_{K} \mathcal{M}_{\chi}\left(c^{\vee}(y)\right)_{\eta}
$$

On the other hand, we have the map

$$
\sigma_{\chi+\phi(y)}: \Gamma\left(G_{\eta}, \mathcal{L}_{\eta}\right) \longrightarrow \Gamma\left(A_{\eta}, \mathcal{M}_{\chi+\phi(y), \eta}\right)
$$

The functional equation we are searching for is

$$
\sigma_{\chi+\phi(y)}=\psi(y) \tau(y, \chi) T_{c^{\vee}(y)}^{*} \circ \sigma_{\chi},
$$

where

$$
\psi(y): \mathcal{M}\left(c^{\vee}(y)\right)_{\eta} \stackrel{\sim}{\rightarrow} \mathcal{O}_{S, \eta}
$$

is a trivialisation of the fibre of $\mathcal{M}$ at $c^{\vee}(y)$, and

$$
\tau(y, \chi): \mathcal{O}_{\chi}\left(c^{\vee}(y)\right)_{\eta} \longrightarrow \mathcal{O}_{S, \eta}
$$

is a section of $\mathcal{O}_{\chi}\left(c^{\vee}(y)\right)_{\eta}^{\otimes-1}$ for each $y \in Y$ and $\chi \in X$, so that $\psi(y) \tau(y, \chi)$ is a section of $\mathcal{M}_{\chi}\left(c^{\vee}(y)\right)_{\eta}^{\otimes-1}$ (recall $\mathcal{M}_{\chi}=\mathcal{M} \otimes \mathcal{O}_{\chi}$ ).

It is a hard fact that

$$
\sigma_{\chi} \neq 0
$$

for all $\chi \in X$; hence, $\tau$ (and $\psi$ ) is uniquely characterised by the functional equation (29). The positivity, bilinearity and symmetry of $\tau$ all follow relatively formally from (29) and $\sigma_{\chi} \neq 0$. Further, $\tau$ is independent of the choice of $\mathcal{L}(\psi$ depends on $\mathcal{L}$ but is independent of the choice of $\mathcal{M})$.

Equation (29) follows formally if we know that $\sigma_{\chi+\phi(y)}$ is proportional to $T_{c^{\vee}(y)}^{*} \circ \sigma_{\chi}$, and this is proved using representations of theta groups. Indeed, we can prove that $\sigma_{\chi}$ factors through an equivariant homomorphism between two irreducible representations with respect to a subgroup of the theta group of $\mathcal{L}$ (isomorphic to the theta group of $\mathcal{M}_{\chi}$, which acts naturally on $\Gamma\left(A_{\eta}, \mathcal{M}_{\chi, \eta}\right)$ ), and similarly for $T_{c^{\vee}(y)}^{*} \circ \sigma_{\chi}$. The nonvanishing of $\sigma_{\chi}$ forces that both factorisations are nonzero, so Schur's lemma gives the proportionality. 


\subsection{PEL degeneration data}

We want to generalise the polarised degeneration data to include endormorphisms and level structures, so that they characterise degenerations of PEL abelian schemes. It turns out that level structures create substantial technical difficulties, which is one of the main technical contributions of Kaiwen Lan. Following Lan's presentation, we separate the data with and without level structures. We use notations from the previous section, and the notations for PEL datum are as in Subsection 3.1.

\subsubsection{Data without level structures}

We begin by defining the degenerating PE abelian varieties.

Definition 5.7. The category $\operatorname{DEG}_{\mathrm{PE}, \mathcal{O}}(R, I)$ has objects $(G, \lambda, i)$ where $(G, \lambda) \in \operatorname{DEG}_{\mathrm{pol}}(R, I)$, and

$$
i: \mathcal{O} \rightarrow \operatorname{End}_{S}(G)
$$

is a ring homomorphism such that

$$
i_{\eta}(b)^{\vee} \circ \lambda_{\eta}=\lambda_{\eta} \circ i_{\eta}\left(b^{*}\right)
$$

for every $b \in \mathcal{O}$, where $i_{\eta}(b)^{\vee}: G_{\eta}^{\vee} \rightarrow G_{\eta}^{\vee}$ is the dual of $i_{\eta}(b)$. The morphisms are isomorphisms respecting all structures.

Remark 5.8. We know that the restriction to the generic fibre is a fully faithful functor from the category of degenerating abelian varieties to that of abelian varieties, which implies that $\lambda_{\eta}: G_{\eta} \rightarrow G_{\eta}^{\vee}$ extends uniquely to a morphism $\lambda: G \rightarrow G^{\vee}$. Thus, it is unambiguous to write $(G, \lambda) \in \operatorname{DEG}_{\mathrm{pol}}(R, I)$. Similarly, we have $\operatorname{End}_{S}(G) \cong \operatorname{End}_{\eta}\left(G_{\eta}\right)$, so the extra data are determined by their restriction to the generic fibre, and the generic fibre is a PE abelian variety by $\mathcal{O}$.

Definition 5.9. The category $\operatorname{DD}_{\mathrm{PE}, \mathcal{O}}(R, I)$ has objects

$$
\left(A, \lambda_{A}, i_{A}, \underline{X}, \underline{Y}, \phi, c, c^{\vee}, \tau\right)
$$

such that $\left(A, \lambda_{A}, \underline{X}, \underline{Y}, \phi, c, c^{\vee}, \tau\right) \in \mathrm{DD}_{\mathrm{pol}}(R, I)$ and

$$
i_{A}: \mathcal{O} \rightarrow \operatorname{End}_{S}(A)
$$

is a ring homomorphism such that $i_{A}(b)^{\vee} \circ \lambda_{A}=\lambda_{A} \circ i_{A}\left(b^{*}\right)$ for every $b \in \mathcal{O}$. The data are required to the additional $\mathcal{O}$-structures in the sense that

(1) $\underline{X}$ and $\underline{Y}$ are étale locally constant sheaves of projective $\mathcal{O}$-modules with structure morphisms $\bar{i}_{\underline{X}}: \mathcal{O} \rightarrow \operatorname{End}_{S}(\underline{X})$ and $i_{\underline{Y}}: \mathcal{O} \rightarrow \operatorname{End}_{S}(\underline{Y}) . \underline{X}$ and $\underline{Y}$ are required to be rationally equivalent as sheaves of $\mathcal{O} \otimes_{\mathbb{Z}} \mathbb{Q}$-modules. Moreover, $\phi: \underline{Y} \rightarrow \underline{X}$ is $\mathcal{O}$-equivariant.

(2) $c: \underline{X} \rightarrow A^{\vee}$ and $c^{\vee}: \underline{Y} \rightarrow A$ are $\mathcal{O}$-equivariant.

(3) The trivialisation $\tau: \mathbf{1}_{\underline{Y} \times} \times \underline{X}, \eta \stackrel{\sim}{\longrightarrow}\left(c^{\vee} \times c\right)^{*} \mathcal{P}_{A, \eta}^{\otimes-1}$ satisfies

$$
\left(i_{\underline{Y}}(b) \times I d_{\underline{X}}\right)^{*} \tau=\left(I d_{\underline{Y}} \times i_{\underline{X}}\left(b^{*}\right)\right)^{*} \tau
$$

for all $b \in \mathcal{O}$; that is, $\tau(b y, \chi)=\tau\left(y, b^{*} \chi\right)$ for $y \in Y$ and $\chi \in X$ if $\underline{X}$ and $\underline{Y}$ are constant.

The morphisms are isomorphisms respecting all structures.

The following theorem follows directly from the functoriality of $F_{\mathrm{pol}}(R, I)$.

Theorem 5.10. There is an equivalence of categories

$$
F_{P E, \mathcal{O}}(R, I): D E G_{P E, \mathcal{O}}(R, I) \rightarrow D D_{P E, \mathcal{O}}(R, I) .
$$


We can strengthen the theorem by adding the Lie algebra condition on both sides. It is the determinant condition in the definition of PEL moduli problems.

Definition 5.11. The category $\operatorname{DEG}_{\mathrm{PE}_{L i e},\left(L \otimes_{\mathbb{Z}} \mathbb{R},\langle\cdot, \cdot\rangle, h\right)}(R, I)$ has objects

$$
(G, \lambda, i) \in \operatorname{DEG}_{\mathrm{PE}, \mathcal{O}}(R, I)
$$

such that $\left(G_{\eta}, \lambda_{\eta}, i_{\eta}\right)$ satisfies the determinant condition specified by $\left(L \otimes_{\mathbb{Z}} \mathbb{R},\langle\cdot, \cdot\rangle, h\right)$; see [18] Definition 1.3.4.1 for definitions. The morphisms are isomorphisms respecting all structures.

Definition 5.12. The category $\mathrm{DD}_{\mathrm{PE}_{L i e},\left(L \otimes_{\mathbb{Z}} \mathbb{R},\langle\cdot, \cdot\rangle, h\right)}(R, I)$ has objects

$$
\left(A, \lambda_{A}, i_{A}, \underline{X}, \underline{Y}, \phi, c, c^{\vee}, \tau\right) \in \mathrm{DD}_{\mathrm{PE}, \mathcal{O}}(R, I)
$$

such that there exists a totally isotropic embedding

$$
\operatorname{Hom}_{\mathbb{R}}(X \otimes \mathbb{R}, \mathbb{R}(1)) \hookrightarrow L \otimes \mathbb{R}
$$

of $\mathcal{O} \otimes \mathbb{R}$-modules with image denoted by $Z_{-2, \mathbb{R}}$, where $X$ is the underlying $\mathcal{O}$-module of $\underline{X}$ and such that $\left(A_{\eta}, \lambda_{A, \eta}, i_{A, \eta}\right)$ satisfies the determinant condition determined by $\left(Z_{-2, \mathbb{R}}^{\perp} / Z_{-2, \mathbb{R}},\langle\cdot, \cdot\rangle, h_{-1}\right)$ induced by the embedding. The morphisms are isomorphisms respecting all of the structures.

Theorem 5.13 (Lan). There is an equivalence of categories

$$
\begin{gathered}
F_{P E_{L i e},\left(L \otimes_{\mathbb{Z}} \mathbb{R},\langle\cdot, \cdot\rangle, h\right)}(R, I): \\
D E G_{P E_{L i e},\left(L \otimes_{\mathbb{Z}} \mathbb{R},\langle\cdot, \cdot\rangle, h\right)}(R, I) \rightarrow D D_{P E_{L i e},\left(L \otimes_{\mathbb{Z}} \mathbb{R},\langle\cdot, \cdot\rangle, h\right)}(R, I) .
\end{gathered}
$$

\subsubsection{Data with level structures}

We will only work with principal level structures in this article. The general level structures can be taken as orbits of principal level structures, and the modification with degeneration data is to take the quotient of the data with principal level structures by certain groups.

We fix an integer $n$ in this section. We assume that the generic point $\eta=\operatorname{Spec}(K)$ is defined over $\operatorname{Spec}\left(\mathcal{O}_{F_{0},(\square)}\right)$, where $F_{0}$ is the reflex field and $\square$ is the set of all primes not dividing $n I_{\text {bad }} D i s c_{\mathcal{O} / \mathbb{Z}}\left[L^{\#}\right.$ : $L]$; see [18] Definition 1.4.1.1 for definitions of these bad primes. In particular, $\operatorname{Spec}\left(\mathcal{O}_{F_{0},(\square)}\right)$ is the maximal base over which the PEL moduli variety is smooth. Moreover, we make the technical assumption that the $\mathcal{O}$-action on $L$ extends to a maximal order in $B$.

All of the morphisms in the category to be defined will be the obvious isomorphisms preserving all of the structures, and we omit the description.

Definition 5.14. The category $\mathrm{DEG}_{\mathrm{PEL}, M_{n}}(R, I)$ has objects

$$
\left(G, \lambda, i,\left(\alpha_{n}, v_{n}\right)\right)
$$

where

$$
(G, \lambda, i) \in \mathrm{DEG}_{\mathrm{PE}_{L i e},\left(L \otimes_{\mathbb{Z}} \mathbb{R},\langle\cdot, \cdot\rangle, h\right)}(R, I) .
$$

Moreover, $\alpha_{n}: L / n L \stackrel{\sim}{\rightarrow} G[n]_{\eta}$ and $v_{n}: \mathbb{Z} / n \mathbb{Z}(1) \stackrel{\sim}{\rightarrow} \mu_{n, \eta}$ are isomorphisms such that they define a level-n structure for $G_{\eta}$ in the sense that

$$
\left(G_{\eta}, \lambda_{\eta}, i_{\eta},\left(\alpha_{n}, v_{n}\right)\right) \in M_{n}(\eta)
$$

as in Definition 3.5. 
Definition 5.15. The category $\mathrm{DD}_{\mathrm{PEL}, M_{n}}(R, I)$ has objects

$$
\left(A, \lambda_{A}, i_{A}, \underline{X}, \underline{Y}, \phi, c, c^{\vee}, \tau,\left[\alpha_{n}^{\natural}\right]\right)
$$

where

$$
\left(A, \lambda_{A}, i_{A}, \underline{X}, \underline{Y}, \phi, c, c^{\vee}, \tau\right) \in \mathrm{DD}_{\mathrm{PE}_{L i e},\left(L \otimes_{\mathbb{Z}} \mathbb{R},\langle\cdot, \cdot\rangle, h\right)}(R, I)
$$

and

$$
\alpha_{n}^{\natural}:=\left(Z_{n}, \varphi_{-2, n},\left(\varphi_{-1, n}, v_{-1, n}\right), \varphi_{0, n}, \delta_{n}, c_{n}, c_{n}^{\vee}, \tau_{n}\right)
$$

is the level structure data with objects to be defined as follows:

(1) $Z_{n}$ is a filtration

$$
0 \subset Z_{n,-2} \subset Z_{n,-1} \subset Z_{n, 0}=L / n L
$$

on $L / n L$, which can be written as the reduction modulo $\mathrm{n}$ of a filtration (of $\mathcal{O} \otimes_{\mathbb{Z}} \hat{\mathbb{Z}}^{\square}$-modules)

$$
0 \subset Z_{-2} \subset Z_{-1} \subset Z_{0}=L \otimes_{\mathbb{Z}} \hat{\mathbb{Z}}^{\square}
$$

on $L \otimes_{\mathbb{Z}} \hat{\mathbb{Z}}^{\square}$ such that $Z$ extends to a filtration $Z_{\mathbb{A}^{\square}}$ on $L \otimes_{\mathbb{Z}} \mathbb{A}^{\square}$ that has the property that it is split (as $\mathcal{O} \otimes_{\mathbb{Z}} \mathbb{A}^{\square}$-modules), $G r_{i}^{Z_{\mathbb{A}^{\square}}}$ is integral for every $\mathrm{i}$; that is, $G r_{i}^{Z_{\mathbb{A}} \square}=M_{i} \otimes_{\mathbb{Z}} \mathbb{A}^{\square}$ for some torsion-free finitely generated $\mathcal{O}$-module $M_{i}$, and $Z_{\mathbb{A}^{\square},-2}$ is the annihilator of $Z_{\mathbb{A}^{\square},-1}$ under the natural pairing $\langle\cdot, \cdot\rangle_{\mathbb{A}^{\square}}$ on $L \otimes_{\mathbb{Z}} \mathbb{A}^{\square}$.

(2) $\varphi_{-1, n}: G r_{-1}^{Z_{n}} \stackrel{\sim}{\rightarrow} A[n]_{\eta}$ and $v_{-1, n}: \mathbb{Z} / n \mathbb{Z}(1) \stackrel{\sim}{\rightarrow} \mu_{n, \eta}$ are isomorphisms such that

$$
\left(A_{\eta}, \lambda_{A, \eta}, i_{A, \eta},\left(\varphi_{-1, n}, v_{-1, n}\right)\right) \in M_{n}(\eta)
$$

with respect to the PEL datum determined by $G r_{-1, n}^{Z_{n}}$, which exists because $Z_{n}$, satisfies the conditions in (1).

(3)

$$
\varphi_{-2, n}: G r_{-2}^{Z_{n}} \stackrel{\sim}{\rightarrow} \underline{H o m}_{\eta}\left((\underline{X} / n \underline{X})_{\eta},(\mathbb{Z} / n \mathbb{Z})(1)\right)
$$

and

$$
\varphi_{0, n}: G r_{0}^{Z_{n}} \stackrel{\sim}{\rightarrow}(\underline{Y} / n \underline{Y})_{\eta}
$$

are isomorphisms which are liftable to some isomorphisms $\varphi_{-2}: G r_{-2}^{Z} \stackrel{\sim}{\rightarrow} \operatorname{Hom}\left(X \otimes_{\mathbb{Z}} \hat{\mathbb{Z}}^{\square}, \hat{\mathbb{Z}}^{\square}(1)\right)$ and $\varphi_{0}: G r_{0}^{Z} \stackrel{\sim}{\rightarrow} Y \otimes_{\mathbb{Z}} \hat{\mathbb{Z}}^{\square}$ over $\bar{\eta}$. Moreover, they are required to satisfy the equation

$$
\left\langle\varphi_{-2, n}(\cdot), \phi \circ \varphi_{0, n}(\cdot)\right\rangle_{c a n}=\langle\cdot, \cdot\rangle_{20, n}
$$

where $\langle\cdot, \cdot\rangle_{\text {can }}: \underline{H o m}_{\eta}\left((\underline{X} / n \underline{X})_{\eta},(\mathbb{Z} / n \mathbb{Z})(1)\right) \times(\underline{X} / n \underline{X})_{\eta} \rightarrow(\mathbb{Z} / n \mathbb{Z})(1)$ is the canonical evaluation pairing, and $\langle\cdot, \cdot\rangle_{20, n}: G r_{-2}^{Z_{n}} \times G r_{0}^{Z_{n}} \rightarrow(\mathbb{Z} / n \mathbb{Z})(1)$ is the pairing induced by $\langle\cdot, \cdot\rangle$ on $L$ (using that (4)

$$
\delta_{n}: \underset{i}{\oplus} G r_{i}^{Z_{n}} \stackrel{\sim}{\rightarrow} L / n L
$$

is a splitting of the filtration $Z_{n}$, which can be lifted to a splitting $\delta: \underset{i}{\oplus G} r_{i}^{Z} \stackrel{\sim}{\rightarrow} L \otimes_{\mathbb{Z}} \hat{\mathbb{Z}}^{\square}$.

$$
c_{n}: \frac{1}{n} \underline{Y}_{\eta} \rightarrow A_{\eta}
$$


and

$$
c_{n}^{\vee}: \frac{1}{n} \underline{X}_{\eta} \rightarrow A_{\eta}^{\vee}
$$

are homomorphisms that lifts $c$ and $c^{\vee}$ over $\eta$; that is, $c_{\eta}=c_{n} \circ\left(\underline{Y}_{\eta} \hookrightarrow \frac{1}{n} \underline{Y}_{\eta}\right)$ and similarly for $c^{\vee}$. They are required to be compatible with the splitting $\delta_{n}$ in the sense that

$$
\left\langle\varphi_{-1, n}(\cdot),\left(\lambda_{A} \circ c_{n}^{\vee}-c_{n} \circ \phi\right) \circ \varphi_{0, n}(\cdot)\right\rangle_{A}=v_{-1, n} \circ\langle\cdot, \cdot\rangle_{10, n}
$$

where $\langle\cdot, \cdot\rangle_{A}: A[n]_{\bar{\eta}} \times A^{\vee}[n]_{\bar{\eta}} \rightarrow \mu_{n, \bar{\eta}}$ is the Weil pairing of $A_{\bar{\eta}}$, and

$$
\langle\cdot, \cdot\rangle_{10, n}: G r_{-1}^{Z_{n}} \times G r_{0}^{Z_{n}} \rightarrow(\mathbb{Z} / n \mathbb{Z})(1)
$$

is the pairing induced by $\delta_{n}$ and the natural pairing $\langle\cdot, \cdot\rangle$ on $L / n L$; that is, $\langle\cdot, \cdot\rangle_{10, n}:=\left\langle\delta_{n}(\cdot), \delta_{n}(\cdot)\right\rangle$ with domain $G r_{-1}^{Z_{n}} \times G r_{0}^{Z_{n}}$. Moreover, they need to satisfy a level-lifting condition compatible with all previous liftings; see [18] Definition 5.2.7.8 for the precise description.

$$
\tau_{n}: \mathbf{1}_{\frac{1}{n}} \times_{S} \underline{X}, \eta \stackrel{\sim}{\longrightarrow}\left(c_{n}^{\vee} \times c_{\eta}\right)^{*} \mathcal{P}_{A, \eta}^{\otimes-1}
$$

is a lifting of $\tau$ in the obvious sense. Similar to (5), it is required to be compatible with $\delta_{n}$ in the sense that

$$
d_{00, n}\left(\varphi_{0, n}(\cdot), \varphi_{0, n}(\cdot)\right)=v_{-1, n} \circ\langle\cdot, \cdot\rangle_{00, n}
$$

where $d_{00, n}: \frac{1}{n} Y / Y \times \frac{1}{n} Y / Y \rightarrow \mu_{n, \bar{\eta}}$ is defined by

$$
d_{00, n}\left(\frac{1}{n} y, \frac{1}{n} y^{\prime}\right):=\tau_{n}\left(\frac{1}{n} y, \phi\left(y^{\prime}\right)\right) \tau_{n}\left(\frac{1}{n} y^{\prime}, \phi(y)\right)^{-1}
$$

for $\frac{1}{n} y, \frac{1}{n} y^{\prime} \in \frac{1}{n} Y$, and $\langle\cdot, \cdot\rangle_{00, n}: G r_{0}^{Z_{n}} \times G r_{0}^{Z_{n}} \rightarrow(\mathbb{Z} / n \mathbb{Z})(1)$ is defined by $\langle\cdot, \cdot\rangle_{00, n}:=\left\langle\delta_{n}(\cdot), \delta_{n}(\cdot)\right\rangle$. They again have to satisfy a level-lifting condition; see [18] Definition 5.2.7.8 for details. Note that we have tacitly used the canonical identification $\frac{1}{n} Y / Y \cong Y / n Y$.

The bracket $\left[\alpha_{n}^{\natural}\right]$ means the equivalence class of $\alpha_{n}^{\natural}$; see [18] Definition 5.2.7.11 for the definition. Essentially, taking the equivalence class is to eliminate the choice of the splitting. The subtlety to define the equivalence is that the complicated relations among the data are described using splittings, and changing splittings will introduce modifications in various data. We only remark that the data $\left(Z_{n}, \varphi_{-2, n},\left(\varphi_{-1, n}, v_{-1, n}\right), \varphi_{0, n}\right)$ are independent of the equivalence class, so the equivalence has effect only on $\left(\delta_{n}, c_{n}, c_{n}^{\vee}, \tau_{n}\right)$.

Remark 5.16. There is redundancy in the above definition, namely, $c$ and $c^{\vee}$ are determined by $c_{n}$ and $c_{n}^{\vee}$, and the same is true for $\tau$.

Theorem 5.17. There is an equivalence of categories

$$
F_{P E L, M_{n}}(R, I): D E G_{P E L, M_{n}}(R, I) \rightarrow D D_{P E L, M_{n}}(R, I) .
$$

\subsubsection{The construction of $F_{P E L, M_{n}}$}

We now explain the meaning of the above complicated data and the construction of $F_{P E L, M_{n}}(R, I)$.

Without loss of generality, we assume that $\underline{X}$ and $\underline{Y}$ are constant with values $X$ and $Y$ from now on. We have already seen how to associate data that characterise the degenerating abelian scheme $G$, together with its PE structures. We now focus on the level structures. The key point is that Mumford construction tells us that $G[n]_{\eta}$ is naturally an extension

$$
0 \rightarrow G^{\natural}[n]_{\eta} \rightarrow G[n]_{\eta} \rightarrow \frac{1}{n} Y / Y \rightarrow 0,
$$


which further justifies the heuristic $G=G^{\natural} / Y$. Moreover, $G^{\natural}$ being a global extension of an abelian variety by an algebraic torus implies that $G^{\natural}[n]_{\eta}$ is also an extension

$$
0 \rightarrow T[n]_{\eta} \rightarrow G^{\natural}[n]_{\eta} \rightarrow A[n]_{\eta} \rightarrow 0
$$

It is clear by naturality that these extensions can be upgraded to extensions in terms of the Tate modules;

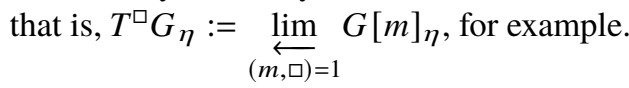

Now if we are given a level-n structure on the generic fibre, we have an isomorphism $\alpha_{n}: L / n L \stackrel{\sim}{\rightarrow}$ $G[n]_{\eta}$ together with an isomorphism $v_{n}: \mathbb{Z} / n \mathbb{Z}(1) \stackrel{\sim}{\rightarrow} \mu_{n, \eta}$, which is compatible with the Weil pairing and liftable to the Tate module. The above two extensions endows a filtration $Z_{n}$ on $L / n L$ through $\alpha_{n}$; that is,

$$
0 \subset Z_{n,-2} \subset Z_{n,-1} \subset Z_{n, 0}=L / n L
$$

such that $\alpha_{n}$ identifies $G r_{-2}^{Z_{n}}, G r_{-1}^{Z_{n}}$ and $G r_{0}^{Z_{n}}$ with $T[n]_{\eta}, A[n]_{\eta}$ and $\frac{1}{n} Y / Y$, respectively. Note that $T[n]_{\eta}=\operatorname{Hom}\left(X / n X, \mu_{n}\right) \stackrel{\nu_{n}}{\cong} \operatorname{Hom}(X / n X, \mathbb{Z} / n \mathbb{Z}(1))$, and we denote the corresponding isomorphisms by

$$
\begin{gathered}
\varphi_{-2, n}: G r_{-2}^{Z_{n}} \stackrel{\sim}{\rightarrow} \operatorname{Hom}(X / n X,(\mathbb{Z} / n \mathbb{Z})(1)), \\
\varphi_{-1, n}: G r_{-1}^{Z_{n}} \stackrel{\sim}{\rightarrow} A[n]_{\eta}
\end{gathered}
$$

and

$$
\varphi_{0, n}: G r_{0}^{Z_{n}} \stackrel{\sim}{\rightarrow}(Y / n Y)_{\eta}
$$

This explains where $\left(Z_{n}, \varphi_{-2, n}, \varphi_{-1, n}, \varphi_{0, n}\right)$ come from. The respective liftability conditions in (1), (2) and (3) of Definition 5.15 correspond to the liftability of the level structure $\alpha_{n}$ and the above extensions. That they satisfy the conditions on Weil pairings in (1), (2) and (3) are general theorems of Grothendieck in SGA 7, where the above two extensions are interpreted as monodromy filtration. The $v_{-1, n}$ in the degeneration data is defined to be $v_{n}$, which is forced by the Weil pairing condition in (2) of Definition 5.15.

We have produced the data $\left(Z_{n}, \varphi_{-2, n},\left(\varphi_{-1, n}, v_{-1, n}\right), \varphi_{0, n}\right)$, which characterise $\alpha_{n}$ up to graded pieces. Now we aim to find more data from which we can recover the complete $\alpha_{n}$. The idea is to introduce auxiliary data that corresponds to splittings of the above two extensions and then take equivalence relations by identifying different splittings.

First, a splitting of the extension

$$
0 \rightarrow T[n]_{\eta} \rightarrow G^{\natural}[n]_{\eta} \rightarrow A[n]_{\eta} \rightarrow 0
$$

is the same as a section of $G^{\natural}[n]_{\eta} \rightarrow A[n]_{\eta}$, which is equivalent to a subgroup scheme $H$ of $G^{\natural}[n]_{\eta}$ that is isomorphic to $A[n]_{\eta}$ through the projection. Let $G_{\eta}^{\natural^{\prime}}:=G_{\eta}^{\natural} / H$, then the quotient map induces

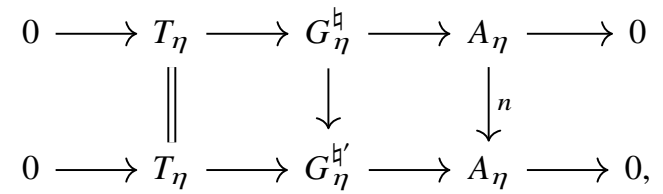


which can be completed into

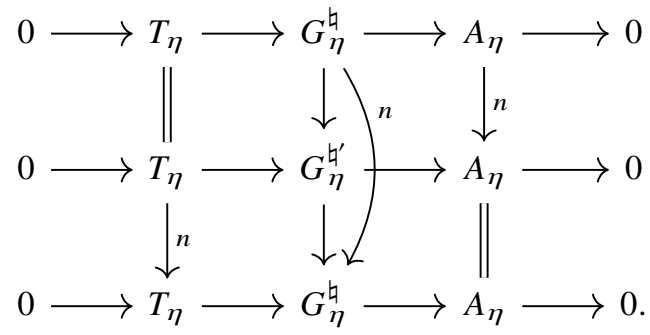

We see that the extension $G_{\eta}^{\natural^{\prime}}$ together with the isogeny

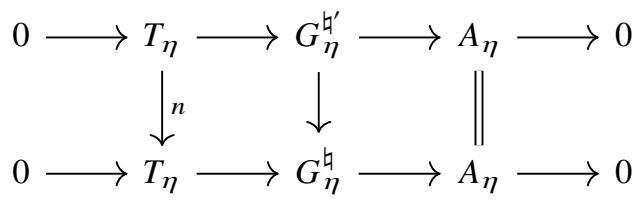

determines the splitting; hence, a splitting of $0 \rightarrow T[n]_{\eta} \rightarrow G^{\natural}[n]_{\eta} \rightarrow A[n]_{\eta} \rightarrow 0$ is equivalent to a diagram as above, which is the same as a lifting $c_{n}: \frac{1}{n} X \rightarrow A_{\eta}^{\vee}$ of $c: X \rightarrow A^{\vee}$ over the generic fibre.

Next, we look at the splitting of

$$
0 \rightarrow G^{\natural}[n]_{\eta} \rightarrow G[n]_{\eta} \rightarrow \frac{1}{n} Y / Y \rightarrow 0 .
$$

From the Mumford quotient $G=G_{\eta}^{\natural} / Y$, it is reasonable to expect that a splitting $\frac{1}{n} Y / Y \rightarrow G[n]_{\eta}$ is equivalent to a lifting

$$
\iota_{n}: \frac{1}{n} Y \longrightarrow G_{\eta}^{\natural}
$$

of the period homomorphism $\imath: Y \rightarrow G_{\eta}^{\natural}$, and this can be proved rigorously. The composition of $\imath_{n}$ with projection to $A_{\eta}$ produces

$$
c_{n}^{\vee}: \frac{1}{n} Y \stackrel{l_{n}}{\longrightarrow} G_{\eta}^{\natural} \stackrel{\pi}{\longrightarrow} A_{\eta},
$$

which lifts $c_{\eta}^{\vee}$ because $l_{n}$ lifts $\imath$ and $c_{\eta}^{\vee}=\pi \circ l$. As we have seen before, such a period homomorphism $\iota_{n}$ is equivalent to a trivialisation of biextensions

$$
\tau_{n}: \mathbf{1}_{\frac{1}{n}} \times_{S} \underline{X}, \eta \stackrel{\sim}{\longrightarrow}\left(c_{n}^{\vee} \times c_{\eta}\right)^{*} \mathcal{P}_{A, \eta}^{\otimes-1}
$$

that lift $\tau$.

We have seen that a splitting of the monodromy filtration on $G[n]_{\eta}$ is equivalent to the data

$$
\left(c_{n}, c_{n}^{\vee}, \tau_{n}\right)
$$

that lift $\left(c_{\eta}, c_{\eta}^{\vee}, \tau\right)$. From the isomorphism $\alpha_{n}: L / n L \stackrel{\sim}{\rightarrow} G[n]_{\eta}$, the splitting on $G[n]_{\eta}$ induces a splitting $\delta_{n}$ of the filtration $Z_{n}$ on $L / n L$, and this finishes the construction of the remaining

$$
\left(\delta_{n}, c_{n}, c_{n}^{\vee}, \tau_{n}\right)
$$

Note that the liftability condition is clearly satisfied. 
To summarise, the data $\left(c_{n}, c_{n}^{\vee}, \tau_{n}\right)$ determine a splitting of the monodromy filtration $W_{n}$ on $G[n]_{\eta}$ - that is, an isomorphism $\zeta_{n}: \underset{i}{\oplus} G r_{i}^{W_{n}} \stackrel{\sim}{\rightarrow} G[n]_{\eta}$ - and the level structure $\alpha_{n}$ can be recovered as

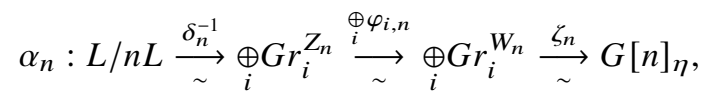

which is liftable by the liftability condition on all intermediate isomorphisms.

The last ingredient is to find characterising conditions for $\alpha_{n}$ to be compatible with the Weil pairing. The key is to use the degeneration data to describe the pairing on $\underset{i}{\oplus} G r_{i}^{W_{n}}$ induced by the Weil pairing on $G[n]_{\eta}$ and the isomorphism $\zeta_{n}$. This is the most difficult part of the construction, as well as one of the main technical contributions of Lan.

We know that the two pairings on $G r_{-2}^{W_{n}} \times G r_{0}^{W_{n}}$ and $G r_{-1}^{W_{n}} \times G r_{-1}^{W_{n}}$ are independent of the splitting because $W_{n,-2}$ is the annihilator of $W_{n,-1}$ and has been determined by Grothendieck as we have already remarked. Because the Weil pairing is alternating, the remaining cases to be determined are $G r_{-1}^{W_{n}} \times G r_{0}^{W_{n}}$ and $G r_{0}^{W_{n}} \times G r_{0}^{W_{n}}$. The result is as follows. The pairing on $G r_{-1}^{W_{n}} \times G r_{0}^{W_{n}}$ is given by

$$
G r_{-1}^{W_{n}} \times G r_{0}^{W_{n}}=A[n]_{\eta} \times \frac{1}{n} Y / Y \rightarrow \mu_{n, \eta},
$$

which sends $\left(a, \frac{1}{n} y\right)$ to

$$
\left\langle a,\left(\lambda_{A, \eta} \circ c_{n}^{\vee}-c_{n} \circ \phi\right)\left(\frac{1}{n} y\right)\right\rangle_{A[n]}
$$

where $\langle\cdot, \cdot\rangle_{A[n]}: A[n]_{\eta} \times A^{\vee}[n]_{\eta} \rightarrow \mu_{n, \eta}$ is the canonical Weil pairing. On the other hand, the pairing

$$
G r_{0}^{W_{n}} \times G r_{0}^{W_{n}}=\frac{1}{n} Y / Y \times \frac{1}{n} Y / Y \rightarrow \mu_{n, \eta}
$$

is given by

$$
\left(\frac{1}{n} y_{1}, \frac{1}{n} y_{2}\right) \longrightarrow \tau_{n}\left(\frac{1}{n} y_{1}, \phi\left(y_{2}\right)\right) \tau_{n}\left(\frac{1}{n} y_{2}, \phi\left(y_{1}\right)\right)^{-1}
$$

We now transform the pairing from $\underset{i}{\oplus} G r_{i}^{W_{n}}$ to $L / n L$ using $\delta_{n}$ and $\varphi_{i, n}$, then the compatibility of $\alpha_{n}$ with the Weil pairing is rephrased in the language of degeneration data, which are exactly the various pairing conditions in Definition 5.15.

Lastly, the equivalence is defined by identifying different splittings $\zeta_{n}$ and $\delta_{n}$ that induce the same $\alpha_{n}$ through (30). This is easily translated into a statement involving only degeneration data; see [18] Definition 5.2.7.11 for details. Because $\zeta_{n}$ is equivalent to $\left(c_{n}, c_{n}^{\vee}, \tau_{n}\right)$, clearly the equivalence only changes $\left(\delta_{n}, c_{n}, c_{n}^{\vee}, \tau_{n}\right)$. This concludes the construction of $F_{P E L, M_{n}}(R, I)$.

\subsection{Toroidal compactifications}

We now review the construction of toroidal compactifications of PEL Shimura varieties. This is in some sense the universal base of a degenerating PEL abelian scheme. We have already seen that degenerating abelian varieties over a Noetherian normal complete affine base is equivalent to a set of degeneration data. The basic idea of the construction of toroidal compactifications is to find the moduli space of the degenerating data and glue them to the Shimura variety.

More precisely, because the degeneration data characterise the degenerating abelian varieties only over a complete base, the moduli space of degeneration data is the completion of the toroidal compactification along the boundary. To obtain the whole compactification, it is necessary to algebraize the complete situation, which is a subtle procedure that we will not review. 
Let us start with the construction of moduli space of degeneration data. We first construct the moduli space of data without the equivalence relation; that is, we want to parametrise the tuple

$$
\left(A, \lambda_{A}, i_{A}, \underline{X}, \underline{Y}, \phi, c, c^{\vee}, \tau, \alpha_{n}^{\natural}\right)
$$

without bracket on $\alpha_{n}^{\natural}$, where

$$
\alpha_{n}^{\natural}:=\left(Z_{n}, \varphi_{-2, n},\left(\varphi_{-1, n}, v_{-1, n}\right), \varphi_{0, n}, \delta_{n}, c_{n}, c_{n}^{\vee}, \tau_{n}\right) .
$$

The moduli space of the degeneration data will be the quotient of this parametrisation space by a group action identifying equivalent data. Without loss of generality, we assume that $\underline{X}$ and $\underline{Y}$ are constant with values $X$ and $Y$ as before.

Because $\left(c, c^{\vee}, \tau\right)$ is determined by $\left(c_{n}, c_{n}^{\vee}, \tau_{n}\right)$, the data we aim to parametrise are

$$
\left(Z_{n},\left(X, Y, \phi, \varphi_{-2, n}, \varphi_{0, n}\right),\left(A, \lambda_{A}, i_{A},\left(\varphi_{-1, n}, v_{-1, n}\right)\right), \delta_{n},\left(c_{n}, c_{n}^{\vee}, \tau_{n}\right)\right),
$$

where

$$
\Phi_{n}:=\left(X, Y, \phi, \varphi_{-2, n}, \varphi_{0, n}\right)
$$

describes the torus part of the degeneration and

$$
\left(A, \lambda_{A}, i_{A},\left(\varphi_{-1, n}, v_{-1, n}\right)\right)
$$

characterises the abelian part, both with level structure specified by $Z_{n}$. Moreover, $\left(c_{n}, c_{n}^{\vee}, \tau_{n}\right)$ contains the information on the extension between abelian and torus parts, the periods and a splitting of the monodromy filtration, which, together with $\delta_{n}$, determine the level structure on the generic fibre of the degenerating abelian variety.

The data

$$
\left(Z_{n},\left(X, Y, \phi, \varphi_{-2, n}, \varphi_{0, n}\right), \delta_{n}\right)
$$

are discrete in nature, and the equivalence class of the tuple is called the cusp label. Indeed, two tuples $\left(Z_{n},\left(X, Y, \phi, \varphi_{-2, n}, \varphi_{0, n}\right), \delta_{n}\right)$ and $\left(Z_{n}^{\prime},\left(X^{\prime}, Y^{\prime}, \phi^{\prime}, \varphi_{-2, n}^{\prime}, \varphi_{0, n}^{\prime}\right), \delta_{n}^{\prime}\right)$ are defined to be equivalent if $Z_{n}=Z_{n}^{\prime}$, and there exist $\mathcal{O}$-equivariant isomorphisms $\gamma_{X}: X^{\prime} \stackrel{\sim}{\rightarrow} X$ and $\gamma_{y}: Y \stackrel{\sim}{\rightarrow} Y^{\prime}$ such that $\phi=\gamma_{X} \phi^{\prime} \gamma_{Y}, \varphi_{-2, n}^{\prime}=\gamma_{X}^{\vee} \varphi_{-2, n}$ and $\varphi_{0, n}^{\prime}=\gamma_{Y} \varphi_{0, n}$. Note that the equivalence classes are independent of the splitting $\delta_{n}$. The cusp labels are essentially equivalence classes of PEL torus. Following Lan's notation, we sometimes abbreviate the notation $\left(Z_{n}, \Phi_{n}, \delta_{n}\right)$ to $\left(\Phi_{n}, \delta_{n}\right)$ for simplicity. This is mostly used in the indexing of various objects.

The abelian part

$$
\left(A, \lambda_{A}, i_{A},\left(\varphi_{-1, n}, v_{-1, n}\right)\right)
$$

is precisely a point of the moduli space of PEL abelian varieties $M_{n}$ with PEL data determined by $G r_{-1}^{Z_{n}}$, which we denote by $M_{n}^{Z_{n}}$. By abuse of notation, we use $A$ to denote the universal PEL abelian variety over $M_{n}^{Z_{n}}$.

Next, the homomorphisms $c_{n}$ and $c_{n}^{\vee}$ are parametrised by the group schemes $\underline{\operatorname{Hom}}_{\mathcal{O}}\left(\frac{1}{n} X, A^{\vee}\right)$ and $\underline{\operatorname{Hom}}_{\mathcal{O}}\left(\frac{1}{n} Y, A\right)$ over $M_{n}^{Z_{n}}$. Recall that $c_{n}$ and $c_{n}^{\vee}$ lift $c$ and $c^{\vee}$, and the latter satisfies the relation $\overline{\lambda_{A} \circ c^{\vee}}=c \circ \phi$, which is equivalent to $\left(c_{n}, c_{n}^{\vee}\right)$ lies in the group scheme

$$
\dddot{C}_{\Phi_{n}}:=\underline{H o m}_{\mathcal{O}}\left(\frac{1}{n} X, A^{\vee}\right) \underset{\underline{H o m}_{\mathcal{O}}}{\times}\left(Y, A^{\vee}\right) \underline{H_{o m}}\left(\frac{1}{n} Y, A\right),
$$

where the first projection map is $c_{n} \rightarrow c_{n} \circ \phi \circ\left(Y \hookrightarrow \frac{1}{n} Y\right)$, and the second one is $c_{n}^{\vee} \rightarrow \lambda_{A} \circ c_{n}^{\vee} \circ(Y \hookrightarrow$ $\left.\frac{1}{n} Y\right)$. 
Further, $\left(c_{n}, c_{n}^{\vee}\right)$ are required to satisfy the relation

$$
\left\langle\varphi_{-1, n}(\cdot),\left(\lambda_{A} \circ c_{n}^{\vee}-c_{n} \circ \phi\right) \circ \varphi_{0, n}(\cdot)\right\rangle_{A}=v_{-1, n} \circ\langle\cdot, \cdot\rangle_{10, n},
$$

and we want to find the subspace of the parametrisation space cut out by this relation. Note that the equation

$$
\left\langle\varphi_{-1, n}(\cdot), b_{\Phi_{n}, \delta_{n}} \circ \varphi_{0, n}(\cdot)\right\rangle_{A}=v_{-1, n} \circ\langle\cdot, \cdot\rangle_{10, n}
$$

defines a liftable homomorphism

$$
b_{\Phi_{n}, \delta_{n}}: \frac{1}{n} Y / Y \longrightarrow A^{\vee}[n]
$$

and the relation (31) is rewritten as

$$
b_{\Phi_{n}, \delta_{n}}=\lambda_{A} \circ c_{n}^{\vee}-c_{n} \circ \phi
$$

Thus, the parametrisation space we are searching for is the fibre at $b_{\Phi_{n}, \delta_{n}}$ of the homomorphism

$$
\partial_{n}: \dddot{C}_{\Phi_{n}} \longrightarrow \underline{\operatorname{Hom}}_{\mathcal{O}}\left(\frac{1}{n} Y / Y, A^{\vee}[n]\right)
$$

that sends $\left(c_{n}, c_{n}^{\vee}\right)$ to $\lambda_{A} \circ c_{n}^{\vee}-c_{n} \circ \phi$. We denote it by

$$
\dddot{C}_{\Phi_{n}, b_{n}}:=\partial_{n}^{-1}\left(b_{\Phi_{n}, \delta_{n}}\right) .
$$

It can be shown that $\dddot{C}_{\Phi_{n}, b_{n}}$ is a trivial torsor with respect to a commutative proper group scheme over $M_{n}^{Z_{n}}$, but it is not necessarily geometrically connected. However, the liftability condition on $\left(c_{n}, c_{n}^{\vee}\right)$ singles out a connected component $C_{\Phi_{n}, b_{n}}$ of $\dddot{C}_{\Phi_{n}, b_{n}}$, which is an abelian scheme. Thus, we see that the tuple

$$
\left(Z_{n},\left(X, Y, \phi, \varphi_{-2, n}, \varphi_{0, n}\right),\left(A, \lambda_{A}, i_{A},\left(\varphi_{-1, n}, v_{-1, n}\right)\right), \delta_{n},\left(c_{n}, c_{n}^{\vee}\right)\right)
$$

is parametrised by

$$
\coprod_{\left(Z_{n}, \Phi_{n}, \delta_{n}\right)} C_{\Phi_{n}, b_{n}}
$$

where $C_{\Phi_{n}, b_{n}}$ is an abelian scheme over $M_{n}^{Z_{n}}$.

The next step is to include $\tau_{n}$ into the parametrisation space. By construction, we have two universal homomorphisms $\left(c_{n}, c_{n}^{\vee}\right)$ over $C_{\Phi_{n}, b_{n}}$. There is a map

$$
\frac{1}{n} Y \times X \longrightarrow \operatorname{Pic}\left(C_{\Phi_{n}, b_{n}}\right)
$$

defined by $\left(\frac{1}{n} y, \chi\right) \rightarrow\left(c_{n}^{\vee}\left(\frac{1}{n} y\right), c_{n}(\chi)\right)^{*} \mathcal{P}_{A}$. The linearity and $\mathcal{O}$-equivariance of $\left(c_{n}, c_{n}^{\vee}\right)$ imply that it descends to a morphism

$$
\Psi_{n}: \dddot{S}_{\Phi_{n}}:=\frac{1}{n} Y \otimes_{\mathbb{Z}} X /\left\{\begin{array}{c}
y \otimes \phi\left(y^{\prime}\right)-y^{\prime} \otimes \phi(y) \\
b \frac{1}{n} y \otimes \chi^{-}\left(\frac{1}{n} y\right) \otimes\left(b^{*} \chi\right)
\end{array}\right\}_{\substack{y, y^{\prime} \in Y \\
\chi \in X, b \in \mathcal{O}}} \longrightarrow \operatorname{Pic}\left(C_{\Phi_{n}, b_{n}}\right)
$$

and such that

$$
\underset{l \in \dddot{S}_{\Phi_{n}}}{\oplus} \Psi_{n}(l)
$$


is an $\mathscr{O}_{C_{\Phi_{n}, b_{n}}}$-algebra; hence, we have

$$
\dddot{\Xi}_{\Phi_{n}, b_{n}}:=\underline{\operatorname{Spec}}_{\mathcal{O}_{C_{\Phi_{n}, b_{n}}}}\left(\underset{l \in \ddot{S}_{\Phi_{n}}}{\oplus} \Psi_{n}(l)\right),
$$

which is a $\dddot{E}_{\Phi_{n}}:=\underline{\operatorname{Hom}}\left(\dddot{S}_{\Phi_{n}}, \mathbf{G}_{m}\right)$-torsor. By construction, there is a universal trivialisation

$$
\tau_{n}: \mathbf{1}_{\frac{1}{n} Y \times X} \stackrel{\sim}{\longrightarrow}\left(c_{n}^{\vee} \times c\right)^{*} \mathcal{P}_{A}^{\otimes-1}
$$

over $\dddot{\Xi}_{\Phi_{n}, b_{n}}$.

Note that $\dddot{E}_{\Phi_{n}}$ is not necessarily a torus because $\dddot{S}_{\Phi_{n}}$ can have torsion elements. However, as explained in [18] Corollary 6.2.3.17, the liftability of $\tau_{n}$ together with the pairing condition in (6) of Definition 5.15 cuts out a subspace $\Xi_{\Phi_{n}, \delta_{n}}$ of $\dddot{\Xi}_{\Phi_{n}, b_{n}}$, which is a

$$
E_{\Phi_{n}}:=\underline{\operatorname{Hom}}\left(S_{\Phi_{n}}, \mathbf{G}_{m}\right)
$$

torsor over $C_{\Phi_{n}, b_{n}}$ where $S_{\Phi_{n}}:=\dddot{S}_{\Phi_{n}, \text { free }}$ is the free part of $\dddot{S}_{\Phi_{n}}$.

Remark 5.18. We have seen that the liftability condition restores connectivity in both $C_{\Phi_{n}, b_{n}}$ and $\Xi_{\Phi_{n}, \delta_{n}}$. This is a subtlety caused by the nontrivial endomorphism structure $\mathcal{O}$. In particular, it does not appear in the Siegel case treated in Faltings-Chai, where the level-n structure is liftable.

Thus, we have seen that the tuple

$$
\left(Z_{n},\left(X, Y, \phi, \varphi_{-2, n}, \varphi_{0, n}\right),\left(A, \lambda_{A}, i_{A},\left(\varphi_{-1, n}, v_{-1, n}\right)\right), \delta_{n},\left(c_{n}, c_{n}^{\vee}\right), \tau_{n}\right)
$$

is parametrised by

$$
\coprod_{\left(Z_{n}, \Phi_{n}, \delta_{n}\right)} \Xi_{\Phi_{n}, \delta_{n}},
$$

where

$$
\Xi_{\Phi_{n}, \delta_{n}}:=\underline{\operatorname{Spec}}_{\sigma_{C_{\Phi_{n}, b_{n}}}}\left(\underset{l \in S_{\Phi_{n}}}{\oplus} \Psi_{n}(l)\right)
$$

is a $E_{\Phi_{n}}$-torsor over the abelian scheme $C_{\Phi_{n}, b_{n}}$ defined over $M_{n}^{Z_{n}}$. This is almost the parametrisation space we are searching for, except that we have not dealt with the positivity condition on $\tau$ (or $\tau_{n}$ ). Indeed, $\Xi_{\Phi_{n}, \delta_{n}}$ is the moduli space of 'degeneration data over the the generic fibre', and it remains to construct the boundary on which the data extend and the positivity condition holds universally.

Recall that the positivity condition for $\tau$ is that the morphism

$$
\tau(y, \phi(y)):\left(c^{\vee}(y) \times c \circ \phi(y)\right)^{*} \mathcal{P}_{A, \eta} \longrightarrow \mathcal{O}_{S, \eta}
$$

extends to $S$ for all $y \in Y$ and that for $y \neq 0$, it factors through the ideal of definition of $S$. We have constructed a universal $\tau_{n}$ over $\Xi_{\Phi_{n}, \delta_{n}}$ by making $\left(c_{n}^{\vee}\left(\frac{1}{n} y\right), c_{n}(\chi)\right)^{*} \mathcal{P}_{A}=\Psi\left(\frac{1}{n} y \otimes \chi\right)$ part of the structure sheaf of the relatively affine scheme $\Xi_{\Phi_{n}, \delta_{n}}$ over $C_{\Phi_{n}, b_{n}}$. There is a natural way to compactify the $E_{\Phi_{n}}{ }^{-}$ torsor $\Xi_{\Phi_{n}, \delta_{n}}$, namely, the toroidal compactification, which produces directions where $\tau$ can extend. However, this is noncanonical and depends on an auxiliary choice of cone decomposition, because there are infinitely many potential directions and it is necessary to make a choice.

More precisely, the cocharacter of $E_{\Phi_{n}}$ is $S_{\Phi_{n}}^{\vee}:=\operatorname{Hom}\left(S_{\Phi_{n}}, \mathbb{Z}\right)$, and the corresponding real vector space $\left(S_{\Phi_{n}}\right)_{\mathbb{R}}^{\vee}$ can be naturally identified with the space of Hermitian pairings $(|\cdot, \cdot|): Y \otimes_{\mathbb{Z}} \mathbb{R} \times Y \otimes_{\mathbb{Z}} \mathbb{R} \rightarrow$ $\mathcal{O} \otimes_{\mathbb{Z}} \mathbb{R}$. Let $\mathbf{P}_{\Phi_{n}} \subset\left(S_{\Phi_{n}}\right)_{\mathbb{R}}^{\vee}$ be the subset of positive semidefinite Hermitian pairings with admissible radical; that is, its radical is the $\mathbb{R}$-span of some direct summand $\mathcal{O}$-submodule of $Y$. Let $\Sigma_{\Phi_{n}}=\left\{\sigma_{j}\right\}$ be 
a rational polyhedral cone decomposition of $\mathbf{P}_{\Phi_{n}}$, and let $\sigma^{\vee}:=\left\{v \in S_{\Phi_{n}} \mid f(v) \geq 0, \forall f \in \sigma\right\}$; then we have the natural toroidal compactification

$$
\bar{\Xi}_{\Phi_{n}, \delta_{n}, \Sigma_{\Phi_{n}}}
$$

of $\Xi_{\Phi_{n}, \delta_{n}}$, obtained by gluing together the relatively affine toroidal varieties

$$
\Xi_{\Phi_{n}, \delta_{n}}\left(\sigma_{j}\right):=\underline{\operatorname{Spec}}_{\sigma_{C_{\Phi_{n}, b_{n}}}}\left(\underset{l \in \sigma_{j}^{\vee}}{\oplus} \Psi_{n}(l)\right)
$$

Alternatively, we can define $\bar{\Xi}_{\Phi_{n}, \delta_{n}, \Sigma_{\Phi_{n}}}$ as $\Xi_{\Phi_{n}, \delta_{n}} \times{ }_{\Phi_{n}} \bar{E}_{\Phi_{n}, \Sigma_{\Phi_{n}}}$, where $\bar{E}_{\Phi_{n}, \Sigma_{\Phi_{n}}}$ is the classical toric variety associated to the cone $\Sigma_{\Phi_{n}}$ (viewed as schemes over $C_{\Phi_{n}, b_{n}}$ ).

Note that the toroidal compactification $\bar{\Xi}_{\Phi_{n}, \delta_{n}, \Sigma_{\Phi_{n}}}$ has the universal property as follows. If $\bar{S}$ is a Noetherian scheme over $C_{\Phi_{n}, b_{n}}$ with $S \subset \bar{S}$ a dense open subscheme, and $S \rightarrow \Xi_{\Phi_{n}, \delta_{n}}$ is a morphism defined over $C_{\Phi_{n}, b_{n}}$, then it extends to a morphism

$$
\bar{S} \longrightarrow \bar{\Xi}_{\Phi_{n}, \delta_{n}, \Sigma_{\Phi_{n}}}
$$

over $C_{\Phi_{n}, b_{n}}$ if and only if for each geometric point $x$ of $\bar{S}$, every dominant morphism $\operatorname{Spec}(V) \rightarrow \bar{S}$ centred at $x$, with $V$ a discrete valuation ring, the associated character

$$
S_{\Phi_{n}} \longrightarrow \mathbb{Z}
$$

lies in the closure $\bar{\sigma}$ for some $\sigma \in \Sigma_{\Phi_{n}}(\sigma$ depends only on $x$ ). The naturally associated character is defined as follows. Let $p: \bar{S} \rightarrow C_{\Phi_{n}, b_{n}}$ be the structure morphism, then we have the commutative diagram

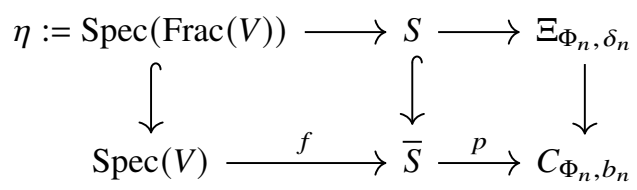

The generic fibre $\left(f^{*} p^{*} \Psi_{n}(l)\right)_{\eta}$ of the line bundle $f^{*} p^{*} \Psi_{n}(l)$ is equipped with a natural trivialisation because it factorises thorough $\Xi_{\Phi_{n}, \delta_{n}}$ as the top row of the diagram shows, while the line bundles $\Psi_{n}(l)$ on $\Xi_{\Phi_{n}, \delta_{n}}$ has a canonical trivialisation by construction. Now under this trivialisation, $f^{*} p^{*} \Psi_{n}(l)$ is identified with a $V$-submodule $I_{l}$ of $\operatorname{Frac}(V)$, we define the desired character $S_{\Phi_{n}} \longrightarrow \mathbb{Z}$ by sending $l$ to the lower bound of the valuation of elements of $I_{l} \subset \operatorname{Frac}(K)$. In other words, $I_{l}=V \pi^{m_{l}} \subset \operatorname{Frac}(V)$ with $\pi \in V$ the uniformiser and $m_{l} \in \mathbb{Z}$, then $l$ is sent to $m_{l}$.

The universal property follows simply by unraveling the definition of toroidal embedding. This is important because it is the ultimate origin of the universal property of toroidal compactifications of Shimura varieties to be discussed in theorem 5.19. The formulation is useful because in the situation we will consider, $I_{l}$ can be directly read off from the degeneration data of a degenerating abelian variety over $V$.

We have now constructed the moduli space of the tuple

$$
\left(Z_{n},\left(X, Y, \phi, \varphi_{-2, n}, \varphi_{0, n}\right),\left(A, \lambda_{A}, i_{A},\left(\varphi_{-1, n}, v_{-1, n}\right)\right), \delta_{n},\left(c_{n}, c_{n}^{\vee}, \tau_{n}\right)\right)
$$

with a specified direction of degeneration, namely,

$$
\coprod_{\left(Z_{n}, \Phi_{n}, \delta_{n}\right)} \bar{\Xi}_{\Phi_{n}, \delta_{n}, \Sigma_{\Phi_{n}}},
$$


over which there is a universal degeneration data, and we would like to find the associated degenerating abelian variety. However, the equivalence between degenerating abelian varieties and degeneration data holds only over a complete base, so the correct object to consider is the completion of $\bar{\Xi}_{\Phi_{n}, \delta_{n}, \Sigma_{\Phi_{n}}}$ along the boundary, which we denote by $\mathfrak{X}_{\Phi_{n}, \delta_{n}, \Sigma_{\Phi_{n}}}$, and there is a universal degenerating abelian variety over $\mathfrak{X}_{\Phi_{n}, \delta_{n}, \Sigma_{\Phi_{n}}}$. More precisely, the equivalence between degeneration data and degenerating abelian varieties is only proved over a complete affine base, and $\bar{\Xi}_{\Phi_{n}, \delta_{n}, \Sigma_{\Phi_{n}}}$ is not affine in general. However, the global degeneration data define degenerating abelian varieties Zariski locally, and it is not hard to use the functoriality of Mumford's construction to glue them together to obtain a global one.

The next step is to quotient out the equivalence relation to find the moduli space of degeneration data. Recall that the equivalence classes $\left[\left(Z_{n}, \Phi_{n}, \delta_{n}\right)\right]$ are called cusp labels, which subsumes the ambiguity caused by equivalence classes of $\alpha_{n}^{\natural}$. Further, we need to take care of the isomorphism classes in the category of degeneration data, and this is described by the action of the automorphism group. We choose a representative $\left(Z_{n}, \Phi_{n}, \delta_{n}\right)$ for each cusp label, and then the automorphism group of the chosen label is

$$
\Gamma_{\Phi_{n}}:=\left\{\left(\gamma_{X}, \gamma_{Y}\right) \in G L_{\mathcal{O}}(X) \times G L_{\mathcal{O}}(Y) \mid \varphi_{-2, n}=\gamma_{X}^{\vee} \varphi_{-2, n}, \varphi_{0, n}=\gamma_{Y} \varphi_{0, n}, \phi=\gamma_{X} \phi \gamma_{Y}\right\}
$$

which acts on $\Xi_{\Phi_{n}, \delta_{n}}$ and $\mathbf{P}_{\Phi_{n}}$. We choose the cone decomposition $\Sigma_{\Phi_{n}}$ to be $\Gamma_{\Phi_{n}}$-admissible; that is, $\gamma \sigma \in \Sigma_{\Phi_{n}}$ for all $\gamma \in \Gamma_{\Phi_{n}}$ and $\sigma \in \Sigma_{\Phi_{n}}$, and the action of $\Gamma_{\Phi_{n}}$ on $\Sigma_{\Phi_{n}}$ has finitely many orbits. Under this condition on $\Sigma_{\Phi_{n}}$, the action of $\Gamma_{\Phi_{n}}$ extends to $\bar{\Xi}_{\Phi_{n}, \delta_{n}, \Sigma_{\Phi_{n}}}$, hence also on $\mathfrak{X}_{\Phi_{n}, \delta_{n}, \Sigma_{\Phi_{n}}}$, and the moduli space of degeneration data is

$$
\underset{\left[\left(Z_{n}, \Phi_{n}, \delta_{n}\right)\right]}{\coprod} \mathfrak{X}_{\Phi_{n}, \delta_{n}, \Sigma_{\Phi_{n}}} / \Gamma_{\Phi_{n}},
$$

where we choose a representative $\left(Z_{n}, \Phi_{n}, \delta_{n}\right)$ for each cusp label, and $\mathfrak{X}_{\Phi_{n}, \delta_{n}, \Sigma_{\Phi_{n}}} / \Gamma_{\Phi_{n}}$ is constructed with respect to this choice. The degenerating abelian variety on $\mathfrak{X}_{\Phi_{n}, \delta_{n}, \Sigma_{\Phi_{n}}}$ descends to $\mathfrak{X}_{\Phi_{n}, \delta_{n}, \Sigma_{\Phi_{n}}} / \Gamma_{\Phi_{n}}$ if a technical condition on $\Sigma_{\Phi_{n}}$ is satisfied (see [18] Condition 6.2.5.25), which we assume from now on.

Now the degenerating PEL abelian variety over $\mathfrak{X}_{\Phi_{n}, \delta_{n}, \Sigma_{\Phi_{n}}} / \Gamma_{\Phi_{n}}$ is a PEL abelian variety over the generic fibre, hence defining a map from the generic fibre of $\mathfrak{X}_{\Phi_{n}, \delta_{n}, \Sigma_{\Phi_{n}}} / \Gamma_{\Phi_{n}}$ to the moduli space $M_{n}$. An appropriate algebraization of these attaching maps will provide gluing maps along neighbourhoods of the boundary of the toroidal compactification of $M_{n}$. Hence, we can glue them together to obtain the toroidal compactification. In order for the gluing process to work well, it is necessary to choose the cones $\Sigma_{\Phi_{n}}$ to be compatible for different $\Phi_{n}$; see [18] Definition 6.3.3.4 for details.

We remark that the algebraization process is very delicate and not canonical. As a consequence, it is difficult to describe the Zariski neighbourhood of the boundary. On the other hand, because the boundary is glued by the algebraization of a formal scheme that we constructed rather explicitly, we have a nice description of the formal neighbourhood of the boundary, which is nothing but $\mathfrak{X}_{\Phi_{n}, \delta_{n}, \Sigma_{\Phi_{n}}} / \Gamma_{\Phi_{n}}$. This also tells us what the boundary looks like, which is simply the support of $\mathfrak{X}_{\Phi_{n}, \delta_{n}, \Sigma_{\Phi_{n}}} / \Gamma_{\Phi_{n}}$. Moreover, the universal property that we described for $\bar{\Xi}_{\Phi_{n}, \delta_{n}, \Sigma_{\Phi_{n}}}$ survives all of the completion, algebraization and gluing procedures and is transformed to a universal property for the toroidal compactification of $M_{n}$.

To summarise, we have the following theorem.

Theorem 5.19 (Lan [18] Theorem 6.4.1.1). To each compatible choice $\Sigma=\left\{\Sigma_{\Phi_{n}}\right\}_{\left[\left(\Phi_{n}, \delta_{n}\right)\right]}$ of admissible smooth rational polyhedral cone decomposition as in [18] Definition 6.3.3.4, there is an associated algebraic stack $M_{n, \Sigma}^{\text {tor }}\left(\right.$ which is a scheme when $n>3$ ) proper and smooth over $\operatorname{Spec}\left(\mathcal{O}_{F_{0},(口)}\right)$, containing $M_{n}$ as an open dense subspace whose complement consists of normal crossing divisors, together with a degenerating abelian variety

$$
\left(G, \lambda, i,\left(\alpha_{n}, v_{n}\right)\right)
$$


over $M_{n, \Sigma}^{\text {tor }}$ as in Definition 5.14, such that we have the following:

(1) The restriction of $\left(G, \lambda, i,\left(\alpha_{n}, v_{n}\right)\right)$ to $M_{n}$ is the universal PEL abelian variety over $M_{n}$.

(2) $M_{n, \Sigma}^{\text {tor }}$ has a stratification by locally closed subschemes

$$
M_{n, \Sigma}^{t o r}=\coprod_{\left[\left(\Phi_{n}, \delta_{n}, \sigma\right)\right]} Z_{\left[\left(\Phi_{n}, \delta_{n}, \sigma\right)\right]}
$$

where $\sigma \in \Sigma_{\Phi_{n}}$ and $\left[\left(\Phi_{n}, \delta_{n}, \sigma\right)\right]$ are the equivalence classes of the tuples $\left(\Phi_{n}, \delta_{n}, \sigma\right)$, which are the obvious refinement of the equivalences used to define cusp labels, namely, by requiring the isomorphisms to preserve $\sigma$; see [18] Definition 6.2.6.1 for details. Note that we suppress $Z_{n}$ in the notation, following Lan.

The formal completion $\left(M_{n, \Sigma}^{\text {tor }}\right)_{Z_{\left[\left(\Phi_{n}, \delta_{n}, \sigma\right)\right]}}^{\wedge}$ of $M_{n, \Sigma}^{\text {tor }}$ along $Z_{\left[\left(\Phi_{n}, \delta_{n}, \sigma\right)\right]}$ is canonically isomorphic to $\mathfrak{X}_{\Phi_{n}, \delta_{n}, \sigma} / \Gamma_{\Phi_{n}, \sigma}$,

$$
\left(M_{n, \Sigma}^{\text {tor }}\right)_{Z_{\left[\left(\Phi_{n}, \delta_{n}, \sigma\right)\right]}}^{\wedge} \mathfrak{X}_{\Phi_{n}, \delta_{n}, \sigma} / \Gamma_{\Phi_{n}, \sigma}
$$

where $\mathfrak{X}_{\Phi_{n}, \delta_{n}, \sigma}$ is the formal completion of

$$
\bar{\Xi}_{\Phi_{n}, \delta_{n}}(\sigma):=\underline{\operatorname{Spec}}_{\sigma_{C_{\Phi_{n}, b_{n}}}}\left(\underset{l \in \sigma^{\vee}}{\oplus} \Psi_{n}(l)\right)
$$

along the boundary $\underline{\operatorname{Spec}}_{\sigma_{C_{\Phi_{n}, b_{n}}}}\left(\underset{l \in \sigma^{\perp}}{\oplus} \Psi_{n}(l)\right)$, with $\sigma^{\perp}:=\left\{x \in S_{\Phi_{n}} \mid\langle x, y\rangle=0, \forall y \in \sigma\right\}$. The scheme $\bar{\Xi}_{\Phi_{n}, \delta_{n}}(\sigma)$ is a relative affine toroidal variety over a $E_{\Phi_{n}}$-torsor over $C_{\Phi_{n}, b_{n}}$, which is an abelian scheme over the PEL moduli space $M_{n}^{Z_{n}}$ with PEL data specified by $G r_{-1}^{Z_{n}}$. Then the strata $Z_{\left[\left(\Phi_{n}, \delta_{n}, \sigma\right)\right]}$ is isomorphic to the support of $\mathfrak{X}_{\Phi_{n}, \delta_{n}, \sigma} / \Gamma_{\Phi_{n}, \sigma}$. If $n>3$, then the action of $\Gamma_{\Phi_{n}, \sigma}$ is trivial, and $\mathfrak{X}_{\Phi_{n}, \delta_{n}, \sigma} / \Gamma_{\Phi_{n}, \sigma} \cong \mathfrak{X}_{\Phi_{n}, \delta_{n}, \sigma}$.

(3) If $S$ is an irreducible Noetherian normal scheme over $\operatorname{Spec}\left(\mathcal{O}_{F_{0},(\square)}\right)$ over which we have a degenerating PEL abelian variety $\left(G^{\dagger}, \lambda^{\dagger}, i^{\dagger},\left(\alpha_{n}^{\dagger}, v_{n}^{\dagger}\right)\right)$ as in Definition 5.14 (with the same PEL data as that of $\left.M_{n}\right)$, then there exists a morphism

$$
S \longrightarrow M_{n, \Sigma}^{t o r}
$$

over Spec $\left(\mathcal{O}_{F_{0},(\square)}\right)$ such that $\left(G^{\dagger}, \lambda^{\dagger}, i^{\dagger},\left(\alpha_{n}^{\dagger}, v_{n}^{\dagger}\right)\right)$ is the pullback of $\left(G, \lambda, i,\left(\alpha_{n}, v_{n}\right)\right)$ if and only if the following condition is satisfied:

For each geometric point $\bar{s}$ of $S$, and any dominant morphism Spec $(V) \rightarrow S$ centered at $\bar{s}$ with $V$ a complete discrete valuation ring, let $\left(G^{\ddagger}, \lambda^{\ddagger}, i^{\ddagger},\left(\alpha_{n}^{\ddagger}, v_{n}^{\ddagger}\right)\right)$ be the pullback of $\left(G^{\dagger}, \lambda^{\dagger}, i^{\dagger},\left(\alpha_{n}^{\dagger}, v_{n}^{\dagger}\right)\right)$ along $\operatorname{Spec}(V) \rightarrow S$, then the theorem on degeneration data provides us with the degeneration data

$$
\left(A^{\ddagger}, \lambda_{A^{\ddagger}}^{\ddagger}, i_{A^{\ddagger}}^{\ddagger}, X^{\ddagger}, Y^{\ddagger}, \phi^{\ddagger}, c^{\ddagger},\left(c^{\vee}\right)^{\ddagger}, \tau^{\ddagger},\left[\left(\alpha_{n}^{\natural}\right)^{\ddagger}\right]\right)
$$

over $V$. Note that $X$ and $Y$ are automatically constant (hence the notation) because $V$ is a complete discrete valuation ring. Moreover, $\left(Z_{n}^{\ddagger}, \Phi_{n}^{\ddagger}\right)$ is determined by $\left[\left(\alpha_{n}^{\natural}\right)^{\ddagger}\right]$. Let $\eta=\operatorname{Spec}(K)$ be the generic fibre of $\operatorname{Spec}(V)$, then the isomorphism

$$
\tau^{\ddagger}:\left(\left(c^{\vee}\right)^{\ddagger} \times c^{\ddagger}\right)^{*} \mathcal{P}_{A^{\ddagger}, \eta} \stackrel{\sim}{\longrightarrow} \boldsymbol{1}_{Y^{\ddagger} \times X^{\ddagger}, \eta}
$$

defines a trivialisation of the generic fibre of the invertible sheaf $\left(\left(c^{\vee}\right)^{\ddagger}(y) \times c^{\ddagger}(\chi)\right)^{*} \mathcal{P}_{A^{\ddagger}}$ on $\operatorname{Spec}(V)$ for each $y \in Y^{\ddagger}, \chi \in X^{\ddagger}$, with which we can identify $\left(\left(c^{\vee}\right)^{\ddagger}(y) \times c^{\ddagger}(\chi)\right)^{*} \mathcal{P}_{A^{\ddagger}}$ with an invertible 
$V$-submodule $I_{y, \chi}$ of $K$. This defines a morphism

$$
\begin{aligned}
Y^{\ddagger} \times X^{\ddagger} & \rightarrow \operatorname{Inv}(V) \\
(y, \chi) & \rightarrow I_{y, \chi}
\end{aligned}
$$

with $\operatorname{Inv}(V)$ the group of invertible $V$-modules (submodules of $K$ ). We can show that it extends to

$$
\frac{1}{n} Y^{\ddagger} \times X^{\ddagger} \longrightarrow \operatorname{Inv}(V)
$$

which descends to a homomorphism

$$
B^{\ddagger}: S_{\Phi_{n}} \longrightarrow \operatorname{Inv}(V)
$$

composed with the natural identification $\operatorname{Inv}(V) \cong \mathbb{Z}$ defined by $\pi^{m} V \longleftrightarrow m$ with $\pi$ the uniformiser. We obtain a homomorphism

$$
v \circ B^{\ddagger}: S_{\Phi_{n}} \longrightarrow \mathbb{Z}
$$

that is an element of $S_{\Phi_{n}}^{\vee}$. The upshot is that we associate an element $v \circ B^{\ddagger} \in S_{\Phi_{n}}^{\vee}$ for each dominant morphism $\operatorname{Spec}(V) \rightarrow S$ centred at $\bar{s}$ with $V$ a complete discrete valuation ring.

Then the condition is that for some choice of $\delta_{n}^{\ddagger}$ making $\left(Z_{n}^{\ddagger}, \Phi_{n}^{\ddagger}, \delta_{n}^{\ddagger}\right)$ a representative of a cusp label, there is a cone $\sigma^{\ddagger} \in \Sigma_{\Phi_{n}^{\ddagger}}$ depending only on $\bar{s}$ such that $v \circ B^{\ddagger} \in \bar{\sigma}^{\ddagger}$ for all those $v \circ B^{\ddagger}$ coming from $a$ dominant $\operatorname{Spec}(V) \rightarrow S$ centred at $\bar{s}$ with $V$ a complete discrete valuation ring, where $\bar{\sigma}^{\ddagger}$ is the closure of $\sigma^{*}$.

\subsection{Partial Frobenius extends to toroidal compactifications}

Now we can prove the main technical results on the extension of partial Frobenius to toroidal compactifications. We follow the notations of Subsection 3.3. In particular, we assume that

$$
p \text { splits completely in the center } F^{c} \text { of } B \text {, }
$$

and the moduli problems

$$
M_{K(n)} / \Delta=\coprod_{\substack{\alpha \in \Omega \\ \delta \in \Lambda}} M_{n}\left(L, \operatorname{Tr}_{\mathcal{O}_{F} / \mathbb{Z}} \circ\left(\alpha \delta\langle\cdot, \cdot\rangle_{F}\right)\right)
$$

are defined over $\mathcal{O}_{F_{0}} \otimes_{\mathbb{Z}} \mathbb{F}_{p}$, where $\Omega$ and $\Lambda$ are fixed sets of representatives of the double quotients

$$
\begin{gathered}
\left(F \otimes \mathbb{A}^{(p \infty)}\right)^{\times}=\coprod_{\alpha \in \Omega}\left(\mathcal{O}_{F} \otimes \mathbb{Z}_{(p)}\right)_{+}^{\times} \alpha\left(\mathcal{O}_{F} \otimes \hat{\mathbb{Z}}^{p}\right)^{\times} \\
\left(\mathcal{O}_{F} \otimes \hat{\mathbb{Z}}^{p}\right)^{\times}=\coprod_{\delta \in \Lambda}\left(\mathcal{O}_{F}\right)_{+}^{\times} \delta\left(v(K(n)) \hat{\mathbb{Z}}^{p, \times}\right) .
\end{gathered}
$$

Let $p=\prod_{i} \mathfrak{p}_{i}$ be the decomposition of $p$ in $\mathcal{O}_{F}$, and we will focus on a single $\mathfrak{p}_{i}$ from now on. We fix a $\xi \in F_{+}^{\times}$satisfying $v_{\mathfrak{p}_{i}}(\xi)=1$ and $v_{\mathfrak{p}_{i^{\prime}}}(\xi)=0$ for $i^{\prime} \neq i$. Recall that the partial Frobenius

$$
F_{\mathfrak{p}_{i}}: M_{K(n)} / \Delta \longrightarrow M_{K(n)} / \Delta
$$

is defined by union of the maps

$$
M_{n}\left(L, \operatorname{Tr}_{\mathcal{O}_{F} / \mathbb{Z}} \circ\left(\alpha \delta\langle\cdot, \cdot\rangle_{F}\right)\right) \rightarrow M_{n}\left(L, \operatorname{Tr}_{\mathcal{O}_{F} / \mathbb{Z}} \circ\left(\alpha^{\prime} \delta^{\prime}\langle\cdot, \cdot\rangle_{F}\right)\right)
$$


with $\alpha^{\prime} \in \Omega, \delta^{\prime} \in \Lambda$ characterised by

$$
\begin{aligned}
& \xi \alpha=\epsilon \alpha^{\prime} \lambda \\
& \lambda \delta=\epsilon_{0} \delta^{\prime} \gamma,
\end{aligned}
$$

where $\epsilon \in\left(\mathcal{O}_{F} \otimes \mathbb{Z}_{(p)}\right)_{+}^{\times}, \lambda \in\left(\mathcal{O}_{F} \otimes \hat{\mathbb{Z}}^{p}\right)^{\times}, \epsilon_{0} \in \mathcal{O}_{F,+}^{\times}$and $\gamma \in\left(v(K(n)) \hat{\mathbb{Z}}^{p, \times}\right)$ as in the above two double quotients of $\left(F \otimes \mathbb{A}^{(p \infty)}\right)^{\times}$and $\left(\mathcal{O}_{F} \otimes \hat{\mathbb{Z}}^{p}\right)^{\times}$. The map is defined by

$$
\left(A, \lambda, i,\left(\alpha_{n}, v_{n}\right)\right) \longrightarrow\left(A^{\prime}, \lambda^{\prime}, i^{\prime},\left(\alpha_{n}^{\prime}, v_{n}^{\prime}\right)\right),
$$

where

$$
A^{\prime}:=A /\left(\operatorname{Ker}(F)\left[\mathfrak{p}_{i}\right]\right),
$$

$i^{\prime}$ is induced by the quotient map $\pi_{\mathfrak{p}_{i}}: A \rightarrow A^{\prime}, \lambda^{\prime}$ is characterised by $\xi \lambda=\pi_{\mathfrak{p}_{i}}^{\vee} \circ \lambda^{\prime} \circ \pi_{\mathfrak{p}_{i}}$, which defines a quasi-isogeny $\lambda^{\prime}, \alpha_{n}^{\prime}=\pi_{\mathfrak{p}_{i}} \circ \alpha_{n}$, and $v_{n}^{\prime}=v_{n} \circ \kappa$.

In the last equality, we fix a set of representatives of $\hat{\mathbb{Z}}^{p, \times} / v(\mathcal{U}(n)) \cong(\mathbb{Z} / n \mathbb{Z})^{\times}$, which defines

$$
v(K(n)) \hat{\mathbb{Z}}^{p, \times}=\coprod_{\kappa} v(K(n)) \kappa
$$

where $\kappa \in \hat{\mathbb{Z}}^{p, \times}$ ranges over the chosen representatives. Then the $\kappa$ in the equality $v_{n}^{\prime}=v_{n} \circ \kappa$ is defined by

$$
\gamma=\beta \kappa,
$$

where $\beta \in v(K(n))$ and $\gamma$ is obtained from the equation $\lambda \delta=\epsilon_{0} \delta^{\prime} \gamma$ as above.

Remark 5.20. The above procedure can be performed to any PEL abelian variety $\left(A, \lambda, i,\left(\alpha_{n}, v_{n}\right)\right)$ defined over a base scheme $S$ over $\mathcal{O}_{F_{0}} \otimes_{\mathbb{Z}} \mathbb{F}_{p}$, and obtains a new PEL abelian variety $\left(A^{\prime}, \lambda^{\prime}, i^{\prime},\left(\alpha_{n}^{\prime}, v_{n}^{\prime}\right)\right)$ over $S$, which is nothing but the map $F_{\mathfrak{p}_{i}}$ on $S$-points.

Now let $\Sigma=\left\{\Sigma_{\alpha \delta}\right\}_{\alpha \in \Omega, \delta \in \Lambda}$, where $\Sigma_{\alpha \delta}$ is a compatible choice of admissible smooth rational polyhedral cone decomposition with respect to the PEL moduli variety $M_{n}\left(L, \operatorname{Tr}_{\mathcal{O}_{F} / \mathbb{Z}} \circ\left(\alpha \delta\langle\cdot, \cdot\rangle_{F}\right)\right)$. Hence, each $\Sigma_{\alpha \delta}$ determines a toroidal compactification $M_{n}\left(L, \operatorname{Tr}_{\mathcal{O}_{F} / \mathbb{Z}} \circ\left(\alpha \delta\langle\cdot, \cdot\rangle_{F}\right)\right)_{\Sigma_{\alpha \delta}}^{t o r}$, the union of which defines the toroidal compactification

$$
\left(M_{K(n)} / \Delta\right)_{\Sigma}^{t o r}:=\coprod_{\substack{\alpha \in \Omega \\ \delta \in \Lambda}} M_{n}\left(L, \operatorname{Tr}_{\mathcal{O}_{F} / \mathbb{Z}} \circ\left(\alpha \delta\langle\cdot, \cdot\rangle_{F}\right)\right)_{\Sigma_{\alpha \delta}}^{\text {tor }} .
$$

Moreover, the union of the strata

$$
M_{n}\left(L, \operatorname{Tr}_{\mathcal{O}_{F} / \mathbb{Z}} \circ\left(\alpha \delta\langle\cdot, \cdot\rangle_{F}\right)\right)_{\Sigma_{\alpha \delta}}^{\text {tor }}=\coprod_{\left[\left(\Phi_{\alpha \delta, n}, \delta_{\alpha \delta, n}, \sigma_{\alpha \delta}\right)\right]} Z_{\left[\left(\Phi_{\alpha \delta, n}, \delta_{\alpha \delta, n}, \sigma_{\alpha \delta}\right)\right]}
$$

defines

$$
\left(M_{K(n)} / \Delta\right)_{\Sigma}^{t o r}=\coprod_{\substack{\alpha \in \Omega \\ \delta \in \Lambda}} \coprod_{\left.\left(\Phi_{\alpha \delta, n}, \delta_{\alpha \delta, n}, \sigma_{\alpha \delta}\right)\right]} Z_{\left[\left(\Phi_{\alpha \delta, n}, \delta_{\alpha \delta, n}, \sigma_{\alpha \delta}\right)\right]} .
$$

We now state the main result of this section.

Theorem 5.21. The partial Frobenius $F_{\mathfrak{p}_{i}}: M_{K(n)} / \Delta \longrightarrow M_{K(n)} / \Delta$ extends to a map

$$
F_{\mathfrak{p}_{i}}:\left(M_{K(n)} / \Delta\right)_{\Sigma}^{\text {tor }} \longrightarrow\left(M_{K(n)} / \Delta\right)_{\Sigma^{\prime}}^{\text {tor }}
$$

where $\Sigma^{\prime}=\left\{\Sigma_{\alpha \delta}^{\prime}\right\}_{\alpha \in \Omega, \delta \in \Lambda}$ is characterised as follows. 
First, for each $\left[\left(Z_{\alpha \delta, n}, \Phi_{\alpha \delta, n}, \delta_{\alpha \delta, n}\right)\right]$ we associate another $\left[\left(Z_{\alpha^{\prime} \delta^{\prime}, n}^{\prime}, \Phi_{\alpha^{\prime} \delta^{\prime}, n}^{\prime}, \delta_{\alpha^{\prime} \delta^{\prime}, n}^{\prime}\right)\right]$ as follows: $\alpha^{\prime} \in \Omega, \delta^{\prime} \in \Lambda$ are determined by $\alpha$ and $\delta$ as in (32) and (33), then

$$
Z_{\alpha^{\prime} \delta^{\prime}, n}^{\prime}=Z_{\alpha \delta, n}
$$

and if $\Phi_{\alpha \delta, n}=\left(X, Y, \phi, \varphi_{-2, n}, \varphi_{0, n}\right)$, we define

$$
\Phi_{\alpha^{\prime} \delta^{\prime}, n}^{\prime}=\left(X \otimes_{\mathcal{O}_{F}} \mathfrak{p}_{i}, Y, \phi^{\prime}, \varphi_{-2, n}^{\prime}, \varphi_{0, n}^{\prime}\right)
$$

where

$$
\begin{gathered}
\varphi_{-2, n}^{\prime}: G r_{-2}^{Z_{\alpha^{\prime} \delta^{\prime}, n}}=G r_{-2}^{Z_{\alpha \delta, n}} \stackrel{\varphi-2, n}{\longrightarrow} \operatorname{Hom}(X / n X,(\mathbb{Z} / n \mathbb{Z})(1)) \\
\stackrel{\sim}{\longrightarrow} \operatorname{Hom}\left(X \otimes \mathfrak{p}_{i} / n\left(X \otimes \mathfrak{p}_{i}\right),(\mathbb{Z} / n \mathbb{Z})(1)\right)
\end{gathered}
$$

and

$$
\varphi_{0, n}^{\prime}: G r_{0}^{Z_{\alpha^{\prime} \delta^{\prime}, n}}=G r_{0}^{Z_{\alpha \delta, n}} \stackrel{\varphi_{0, n}}{\longrightarrow} Y / n Y .
$$

Further, $\phi^{\prime}$ is defined by the diagram

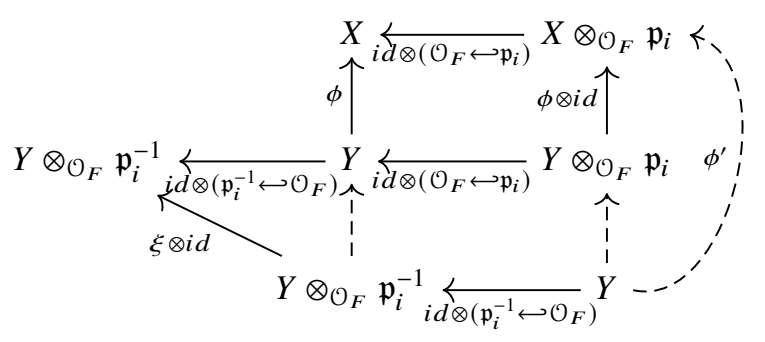

Now for every $\sigma \in \Sigma_{\Phi_{\alpha \delta, n}}$, we associate $\sigma^{\prime} \in \Sigma_{\Phi_{\alpha^{\prime} \delta^{\prime}, n}}^{\prime}$ by $(\otimes \mathbb{R}$ of $)$ the pullback map $S_{\Phi_{\alpha \delta}}^{\vee} \rightarrow S_{\Phi_{\alpha^{\prime} \delta^{\prime}}}^{\vee}$ (recall that $S_{\Phi_{\alpha \delta}}^{\vee}$ is the set of bilinear pairings $Y \times X \rightarrow \mathbb{Z}$ that are $\mathcal{O}$-compatible and becomes symmetric Hermitian once we pull back to $Y \times Y$ along $\phi$ ) induced by the natural map

$$
Y \times\left(X \otimes \mathfrak{p}_{i}\right) \longrightarrow Y \times X
$$

More precisely, the pullback map induces an isomorphism $\left(S_{\Phi_{\alpha \delta} \delta}\right)_{\mathbb{R}}^{\vee} \stackrel{\sim}{\rightarrow}\left(S_{\Phi_{\alpha^{\prime} \delta^{\prime}}}\right)_{\mathbb{R}}^{\vee}$ preserving positive semidefinite pairings, hence defining an identification $\Sigma_{\Phi_{\alpha \delta, n}} \stackrel{\sim}{\rightarrow} \Sigma_{\Phi_{\alpha^{\prime} \delta^{\prime}, n}^{\prime}}^{\prime}$, and we define $\Sigma_{\alpha^{\prime} \delta^{\prime}}^{\prime}=$ $\left\{\sum_{\Phi_{\alpha^{\prime} \delta^{\prime}, n}^{\prime}}^{\prime}\right\}_{\left[\left(\Phi_{\alpha^{\prime} \delta^{\prime}, n}^{\prime}, \delta_{\alpha^{\prime} \delta^{\prime}, n}^{\prime}\right)\right]}$

Moreover, with the association as described above, the map $F_{p_{i}}$ sends $Z_{\left[\left(\Phi_{\alpha \delta, n}, \delta_{\alpha \delta, n}, \sigma_{\alpha \delta}\right)\right]}$ to $Z_{\left[\left(\Phi_{\alpha^{\prime} \delta^{\prime}, n}^{\prime}, \delta_{\alpha^{\prime} \delta^{\prime}, n}^{\prime}, \sigma_{\alpha^{\prime} \delta^{\prime}}^{\prime}\right)\right]}$.

Proof. It is enough to prove that $F_{\mathfrak{p}_{i}}$ extends to the toroidal compactification on each component; that is,

$$
M_{n}\left(L, \operatorname{Tr}_{\mathcal{O}_{F} / \mathbb{Z}} \circ\left(\alpha \delta\langle\cdot, \cdot\rangle_{F}\right)\right) \rightarrow M_{n}\left(L, \operatorname{Tr}_{\mathcal{O}_{F} / \mathbb{Z}} \circ\left(\alpha^{\prime} \delta^{\prime}\langle\cdot, \cdot\rangle_{F}\right)\right)
$$

extends to a morphism

$$
M_{n}\left(L, \operatorname{Tr}_{\mathcal{O}_{F} / \mathbb{Z}} \circ\left(\alpha \delta\langle\cdot, \cdot\rangle_{F}\right)\right)_{\Sigma_{\alpha \delta}}^{t o r} \rightarrow M_{n}\left(L, \operatorname{Tr}_{\mathcal{O}_{F} / \mathbb{Z}} \circ\left(\alpha^{\prime} \delta^{\prime}\langle\cdot, \cdot\rangle_{F}\right)\right)_{\Sigma_{\alpha^{\prime} \delta^{\prime}}^{\prime}}^{t o r}
$$

and maps strata to the expected ones. This reduces the question to toroidal compactifications of Kottwitz's PEL moduli varieties, and we can apply the general machinery of Lan and, in particular, the universal property in Theorem 5.19. 
The idea is very simple. Let $G$ be the universal semi-abelian variety (with extra structures on the open part) over $M_{n}\left(L, \operatorname{Tr}_{\mathcal{O}_{F} / \mathbb{Z}} \circ\left(\alpha \delta\langle\cdot, \cdot\rangle_{F}\right)\right)_{\Sigma_{\alpha \delta}}^{t o r}$. Because the partial Frobenius sends $A$ to $A /\left(\operatorname{Ker}(F)\left[\mathfrak{p}_{i}\right]\right)$, it is natural to extend the map on semi-abelian varieties by the same formula $G \rightarrow G /\left(\operatorname{Ker}(F)\left[\mathfrak{p}_{i}\right]\right)$ (and take care of the extra structures), and this simple idea does indeed work. More precisely, we can define a new semi-abelian scheme

$$
G /\left(\operatorname{Ker}(F)\left[\mathfrak{p}_{i}\right]\right)
$$

(with extra structures on the part by definition of partial Frobenius) over $M_{n}\left(L, \operatorname{Tr}_{\mathcal{O}_{F} / \mathbb{Z}} \circ\left(\alpha \delta\langle\cdot, \cdot\rangle_{F}\right)\right)_{\Sigma_{\alpha \delta}}^{t o r}$, and we would like it to be the pullback of the universal semi-abelian variety over $M_{n}\left(L, \operatorname{Tr}_{\mathcal{O}_{F} / \mathbb{Z}^{\circ}}\right.$ 。 $\left.\left(\alpha^{\prime} \delta^{\prime}\langle\cdot, \cdot\rangle_{F}\right)\right)_{\Sigma_{\alpha^{\prime} \delta^{\prime}}^{\prime}}^{t o r}$ through a morphism

$$
M_{n}\left(L, \operatorname{Tr}_{\mathcal{O}_{F} / \mathbb{Z}} \circ\left(\alpha \delta\langle\cdot, \cdot\rangle_{F}\right)\right)_{\Sigma_{\alpha \delta}}^{t o r} \rightarrow M_{n}\left(L, \operatorname{Tr}_{\mathcal{O}_{F} / \mathbb{Z}} \circ\left(\alpha^{\prime} \delta^{\prime}\langle\cdot, \cdot\rangle_{F}\right)\right)_{\Sigma_{\alpha^{\prime} \delta^{\prime}}^{\prime}}^{t o r}
$$

The universal property of $M_{n}\left(L, \operatorname{Tr}_{\mathcal{O}_{F} / \mathbb{Z}} \circ\left(\alpha^{\prime} \delta^{\prime}\langle\cdot, \cdot\rangle_{F}\right)\right)_{\Sigma_{\alpha^{\prime} \delta^{\prime}}^{\prime o r}}^{t r}$ tells us exactly when this happens, and all we need to do is to verify that the semi-abelian variety ${ }^{\alpha} \delta /\left(\operatorname{Ker}(F)\left[\mathfrak{p}_{i}\right]\right)$ satisfies the condition of the universal property. This amounts to finding the period of the degenerating abelian variety or, more precisely, the bilinear pairing $v \circ B^{\ddagger} \in S_{\Phi_{\alpha^{\prime} \delta^{\prime}}}^{\vee}$ in the notation of Theorem 5.19, which is defined through the assoicated degeneration data. Hence, we need to find the degeneration data of $G /\left(\operatorname{Ker}(F)\left[\mathfrak{p}_{i}\right]\right)$. More precisely, given the degeneration data of $G$, we aim to write the degeneration data of $G /\left(\operatorname{Ker}(F)\left[\mathfrak{p}_{i}\right]\right)$ in terms of that of $G$; that is, to translate the map $G \rightarrow G /\left(\operatorname{Ker}(F)\left[\mathfrak{p}_{i}\right]\right)$ to the language of degeneration data (in a suitable formal setting).

First, note that the restriction of $G /\left(\operatorname{Ker}(F)\left[\mathfrak{p}_{i}\right]\right)$ to the open stratum is simply the old $A^{\prime}$, and the definition of partial Frobenius already tells us that it comes with the PEL structure; that is, we have a degenerating PEL family

$$
\left(G^{\prime}, \lambda^{\prime}, i^{\prime},\left(\alpha_{n}^{\prime}, v_{n}^{\prime}\right)\right)
$$

over $M_{n}\left(L, T r_{\mathcal{O}_{F} / \mathbb{Z}} \circ\left(\alpha \delta\langle\cdot, \cdot\rangle_{F}\right)\right)_{\Sigma_{\alpha \delta}}^{t o r}$, because all of the extra data are defined on the generic open part (although $\lambda^{\prime}$ and $i^{\prime}$ extends to the whole base by formal argument).

Let us specialise the setting of the universal property in Theorem 5.19 to our case. Let us fix a geometric point $\bar{s}$ of $M_{n}\left(L, \operatorname{Tr}_{\mathcal{O}_{F} / \mathbb{Z}} \circ\left(\alpha \delta\langle\cdot, \cdot\rangle_{F}\right)\right)_{\Sigma_{\alpha \delta}}^{t o r}$, and we assume that it lies in the strata $Z_{\left[\left(\Phi_{\alpha \delta, n}, \delta_{\alpha \delta, n}, \sigma_{\alpha \delta}\right)\right]}$. Let $V$ be a complete discrete valuation ring, and we are given a dominant morphism $\operatorname{Spec}(V) \rightarrow M_{n}\left(L, \operatorname{Tr}_{\mathcal{O}_{F} / \mathbb{Z}} \circ\left(\alpha \delta\langle\cdot, \cdot\rangle_{F}\right)\right)_{\Sigma_{\alpha \delta}}^{t o r}$ centred at $\bar{s}$, and

$$
\left(G^{\dagger}, \lambda^{\dagger}, i^{\dagger},\left(\alpha_{n}^{\dagger}, v_{n}^{\dagger}\right)\right)
$$

the pullback of $\left(G^{\prime}, \lambda^{\prime}, i^{\prime},\left(\alpha_{n}^{\prime}, v_{n}^{\prime}\right)\right)$ to $\operatorname{Spec}(V)$.

It is an easy observation that $G^{\dagger} \cong G_{\operatorname{Spec}(V)} /\left(\operatorname{Ker}(F)\left[\mathfrak{p}_{i}\right]\right)$; that is, we can first pull back the universal semi-abelian $G$ and then apply the partial Frobenius operation, and similarly for the extra structures.

Let

$$
\left(A, \lambda_{A}, i_{A}, X, Y, \phi, c, c^{\vee}, \tau,\left[\alpha_{n}^{\natural}\right]\right)
$$

be the degeneration data associated to $\left(G, \lambda, i,\left(\alpha_{n}, v_{n}\right)\right)_{\operatorname{Spec}(V)}$. Because $V$ is centred at $\bar{s}$, which lies in $Z_{\left[\left(\Phi_{\alpha \delta, n}, \delta_{\alpha \delta, n}, \sigma_{\alpha \delta}\right)\right]}$, we see that the torus part of the degeneration data is the same as $\Phi_{\alpha \delta, n}$, and similarly for $\delta_{\alpha \delta, n}$ (if we choose a representative $\left(Z_{\alpha \delta, n}, \Phi_{\alpha \delta, n}, \delta_{\alpha \delta, n}\right)$ of the cusp label $\left.\left[\left(Z_{\alpha \delta, n}, \Phi_{\alpha \delta, n}, \delta_{\alpha \delta, n}\right)\right]\right)$. Moreover, $\sigma_{\alpha \delta}$ determines the range of $\tau_{n}$ in $\alpha_{n}^{\natural}$.

We want to write the degeneration data

$$
\left(A^{\dagger}, \lambda_{A^{\dagger}}^{\dagger}, i_{A^{\dagger}}^{\dagger}, X^{\dagger}, Y^{\dagger}, \phi^{\dagger}, c^{\dagger},\left(c^{\vee}\right)^{\dagger}, \tau^{\dagger},\left[\left(\alpha_{n}^{\natural}\right)^{\dagger}\right]\right)
$$


of $\left(G^{\dagger}, \lambda^{\dagger}, i^{\dagger},\left(\alpha_{n}^{\dagger}, v_{n}^{\dagger}\right)\right)$ in terms of that of $\left(G, \lambda, i,\left(\alpha_{n}, v_{n}\right)\right)_{\operatorname{Spec}(V)}$. More precisely, our aim is to describe $v \circ B^{\dagger}$ in terms of $v \circ B$, and it is enough to describe certain parts of the degeneration data for our purpose, as in the next proposition.

Recall that $B$ is a homomorphism $S_{\Phi_{n}} \rightarrow \operatorname{Inv}(V)$ induced by

$$
\frac{1}{n} Y \times X \longrightarrow \operatorname{Inv}(V)
$$

which is

$$
(y, \chi) \longrightarrow I_{y, \chi}:=\left(c^{\vee}(y) \times c(\chi)\right)^{*} P_{A} \stackrel{\tau}{\subset} K:=\operatorname{Frac}(V)
$$

when restricted to $Y \times X$, and similarly for $B^{\dagger}$. By Proposition 5.22, we have that

$$
\tau^{\dagger}=\left.\tau\right|_{Y^{\dagger} \times X^{\dagger}}
$$

under the natural inclusion

$$
Y^{\dagger} \times X^{\dagger}=Y \times\left(X \otimes_{\mathcal{O}_{F}} \mathfrak{p}_{i}\right) \hookrightarrow Y \times X
$$

and identification

$$
\left(\left(c^{\vee}\right)^{\dagger} \times c^{\dagger}\right)^{*} P_{A^{\dagger}} \cong\left(\left(c^{\vee} \times c\right)^{*} P_{A}\right)_{Y^{\dagger} \times X^{\dagger}}
$$

Therefore, by abuse of notation (viewing $B$ as bilinear forms on $\frac{1}{n} Y \times X$, and similarly for $B^{\dagger}$ ),

$$
\left.B^{\dagger}\right|_{Y^{\dagger} \times X^{\dagger}}=\left.B\right|_{Y^{\dagger} \times X^{\dagger}},
$$

where the second restriction is induced by

$$
Y^{\dagger} \times X^{\dagger}=Y \times\left(X \otimes_{\mathcal{O}_{F}} \mathfrak{p}_{i}\right) \hookrightarrow Y \times X
$$

We now have obtained

$$
\left.v \circ B^{\dagger}\right|_{Y^{\dagger} \times X^{\dagger}}=\left.v \circ B\right|_{Y^{\dagger} \times X^{\dagger}}
$$

which means that

$$
n\left(v \circ B^{\dagger}\right)=n\left(\left.v \circ B\right|_{Y^{\dagger} \times X^{\dagger}}\right) .
$$

By construction, we have (for all dominant morphisms $\operatorname{Spec}(V) \rightarrow M_{n}\left(L, \operatorname{Tr}_{\mathcal{O}_{F} / \mathbb{Z}} \circ\left(\alpha \delta\langle\cdot, \cdot\rangle_{F}\right)\right)_{\Sigma_{\alpha \delta}}^{t o r}$ centred at $\bar{s}$ )

$$
v \circ B \in \sigma_{\alpha \delta},
$$

and by the definition of $\sigma_{\alpha^{\prime} \delta^{\prime}}^{\prime}$ as in the statement of the theorem, we have

$$
n\left(v \circ B^{\dagger}\right)=n\left(\left.v \circ B\right|_{Y^{\dagger} \times X^{\dagger}}\right) \in \sigma_{\alpha^{\prime} \delta^{\prime}}^{\prime},
$$

which implies that (for all dominant morphism $\operatorname{Spec}(V) \rightarrow M_{n}\left(L, \operatorname{Tr}_{\mathcal{O}_{F} / \mathbb{Z}} \circ\left(\alpha \delta\langle\cdot, \cdot\rangle_{F}\right)\right)_{\Sigma_{\alpha \delta}}^{t o r}$ centred at $\bar{s})$

$$
v \circ B^{\dagger} \in \sigma_{\alpha^{\prime} \delta^{\prime}}^{\prime}
$$

because $\sigma_{\alpha^{\prime} \delta^{\prime}}^{\prime}$ is a cone. This finishes the verification of the universal property and also the proof of Theorem 5.21, modulo the following Proposition 5.22. 
Proposition 5.22. Let $V$ be a complete discrete valuation ring that is defined over $\mathcal{O}_{F_{0}} \otimes_{\mathbb{Z}} \mathbb{F}_{p}$ with generic fibre $\eta$, and

$$
\left(G, \lambda, i,\left(\alpha_{n}, v_{n}\right)\right)
$$

is a PEL degenerating abelian variety over $\operatorname{Spec}(V)$; that is, $\left(G, \lambda, i,\left(\alpha_{n}, v_{n}\right)\right) \in D E G_{P E L, M_{n}}(V)$ as in Definition 5.14. Let

$$
\left(G^{\prime}, \lambda^{\prime}, i^{\prime},\left(\alpha_{n}^{\prime}, v_{n}^{\prime}\right)\right) \in D E G_{P E L, M_{n}}(V)
$$

be the degenerating abelian variety defined by

$$
G^{\prime}:=G /\left(\operatorname{Ker}(F)\left[\mathfrak{p}_{i}\right]\right)
$$

and the rest of the structures obtained by applying the partial Frobenius to the generic fibre $\left(G, \lambda, i,\left(\alpha_{n}, v_{n}\right)\right)_{\eta}$ that is a PEL abelian variety, as in Remark 5.20.

Let

$$
\left(A, \lambda_{A}, i_{A}, X, Y, \phi, c, c^{\vee}, \tau,\left[\alpha_{n}^{\natural}\right]\right)
$$

be the degeneration data of $\left(G, \lambda, i,\left(\alpha_{n}, v_{n}\right)\right)$ and

$$
\left(A^{\prime}, \lambda_{A^{\prime}}^{\prime}, i^{\prime} A^{\prime}, X^{\prime}, Y^{\prime}, \phi^{\prime}, c^{\prime}, c^{\prime \vee}, \tau^{\prime},\left[\alpha_{n}^{\prime \natural}\right]\right)
$$

be the degeneration data of $\left(G^{\prime}, \lambda^{\prime}, i^{\prime},\left(\alpha_{n}^{\prime}, v_{n}^{\prime}\right)\right)$. Then

$$
\left(A^{\prime}, \lambda_{A^{\prime}}^{\prime}, i_{A^{\prime}}^{\prime},\left(\varphi_{-1, n}^{\prime}, v_{-1, n}^{\prime}\right)\right)
$$

is obtained by applying the partial Frobenius to $\left(A, \lambda_{A}, i_{A},\left(\varphi_{-1, n}, v_{-1, n}\right)\right)$ over $V$,

$$
\begin{aligned}
& Z_{n}^{\prime}=Z_{n}, \\
& X^{\prime}=X \otimes_{\mathcal{O}_{F}} \mathfrak{p}_{i}, \\
& Y^{\prime}=Y, \\
& \varphi_{-2, n}^{\prime}: G r_{-2}^{Z_{n}^{\prime}}=G r_{-2}^{Z_{n}} \stackrel{\varphi_{-2, n}}{\longrightarrow} \operatorname{Hom}(X / n X,(\mathbb{Z} / n \mathbb{Z})(1)) \\
& \stackrel{\sim}{\longrightarrow} \operatorname{Hom}\left(X \otimes \mathfrak{p}_{i} / n\left(X \otimes \mathfrak{p}_{i}\right),(\mathbb{Z} / n \mathbb{Z})(1)\right), \\
& \varphi_{0, n}^{\prime}: G r_{0}^{Z_{n}^{\prime}}=G r_{0}^{Z_{n}} \stackrel{\varphi_{0, n}}{\longrightarrow} Y / n Y,
\end{aligned}
$$

and $\phi^{\prime}$ is defined by the diagram

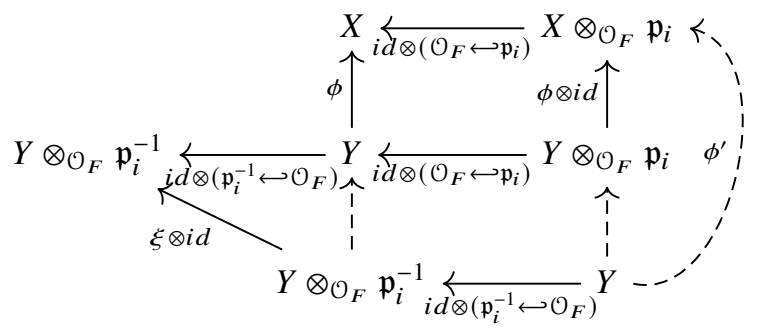


Moreover, we have

$$
\begin{gathered}
c^{\prime}: X^{\prime}=X \otimes_{\mathcal{O}_{F}} \mathfrak{p}_{i} \stackrel{c \otimes i d}{\longrightarrow} A^{\vee} \otimes_{\mathcal{O}_{F}} \mathfrak{p}_{i} \stackrel{\pi \otimes i d}{\longrightarrow} A^{\vee \prime} \otimes_{\mathcal{O}_{F}} \mathfrak{p}_{i} \cong A^{\prime \vee} \\
c^{\prime \vee}: Y^{\prime}=Y \stackrel{c^{\vee}}{\longrightarrow} A \stackrel{\pi}{\longrightarrow} A^{\prime},
\end{gathered}
$$

where $\pi: A \rightarrow A^{\prime}:=A /\left(\operatorname{Ker}(F)\left[\mathfrak{p}_{i}\right]\right)$ is the projection map and the isomorphism $A^{\vee \prime} \otimes_{\mathcal{O}_{F}} \mathfrak{p}_{i} \cong A^{\prime \vee}$ is as in Lemma 3.15.

Last and most important, there is a canonical isomorphism

$$
\left(c^{\prime \vee} \times c^{\prime}\right)^{*} P_{A^{\prime}} \cong\left(\left(c^{\vee} \times c\right)^{*} P_{A}\right)_{Y^{\prime} \times X^{\prime}}
$$

where the pullback to $Y^{\prime} \times X^{\prime}$ is through the natural injection

$$
Y^{\prime} \times X^{\prime}=Y \times\left(X \otimes_{\mathcal{O}_{F}} \mathfrak{p}_{i}\right) \hookrightarrow Y \times X
$$

induced by $X \otimes_{\mathcal{O}_{F}} \mathfrak{p}_{i} \hookrightarrow X \otimes_{\mathcal{O}_{F}} \mathcal{O}_{F} \cong X$. Now $\tau^{\prime}$ is identified as

$$
\tau^{\prime}: 1_{Y^{\prime} \times X^{\prime}, \eta} \stackrel{\left.\tau\right|_{Y^{\prime} \times X^{\prime}}}{\longrightarrow}\left(\left(c^{\vee} \times c\right)^{*} \mathcal{P}_{A, \eta}^{\otimes-1}\right)_{Y^{\prime} \times X^{\prime}} \cong\left(c^{\vee} \times c^{\prime}\right)^{*} P_{A^{\prime}, \eta}^{\otimes-1} .
$$

Proof. We first fix the notation as follows. For any commutative group scheme $H$ over $V$ with an action of $\mathcal{O}$, we denote

$$
H^{\left(\mathfrak{p}_{i}\right)}:=H /\left(\operatorname{Ker}(F)\left[\mathfrak{p}_{i}\right]\right),
$$

where $F$ is the relative Frobenius.

We begin by showing that taking the partial Frobenius quotient commutes with the Raynaud extension; that is, we have the following.

\section{Lemma 5.23.}

$$
G^{\left(\mathfrak{p}_{i}\right), \natural} \cong G^{\natural,\left(\mathfrak{p}_{i}\right)} .
$$

Proof of the Lemma. Recall that $G^{\natural}$ is characterised as the unique global extension of an abelian variety by a torus whose formal completion along the maximal ideal of $V$ is the same as that of $G$; that is, $G^{\natural}$ sits in an extension

$$
0 \rightarrow T \rightarrow G^{\natural} \rightarrow A \rightarrow 0
$$

with $T$ a torus and $A$ an abelian scheme over $V$, which satisfies $G_{\text {for }}^{\natural} \cong G_{\text {for }}$. We have a commutative diagram for the relative Frobenius $F$

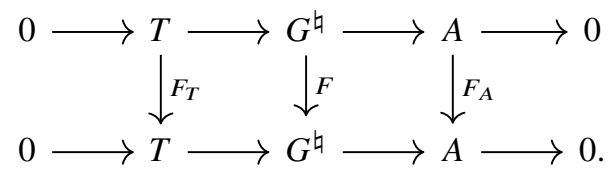

We observe that the relative Frobenius is a faithfully flat morphism on smooth schemes, which in particular shows that $F_{T}$ is surjective as a morphism in the category of fppf sheaves of abelian groups. Then the associated long exact sequence of the diagram tells us that we have a short exact sequence

$$
0 \rightarrow \operatorname{Ker}\left(F_{T}\right) \rightarrow \operatorname{Ker}(F) \rightarrow \operatorname{Ker}\left(F_{A}\right) \rightarrow 0
$$

of finite flat group schemes over $V$. Because the diagram is $\mathcal{O}$-equivariant, so is the short exact sequence of $\operatorname{Ker}(F)$. From the observation that $\operatorname{Ker}(F)$ is killed by $p$ (and so are the other two groups), we 
see that $\operatorname{Ker}(F)=\prod_{i} \operatorname{Ker}(F)\left[\mathfrak{p}_{i}\right]$ (and similarly for the other two), and the above short exact sequence decomposes into a product of short exact sequences

$$
0 \rightarrow \operatorname{Ker}\left(F_{T}\right)\left[\mathfrak{p}_{i}\right] \rightarrow \operatorname{Ker}(F)\left[\mathfrak{p}_{i}\right] \rightarrow \operatorname{Ker}\left(F_{A}\right)\left[\mathfrak{p}_{i}\right] \rightarrow 0 .
$$

Now the commutative diagram

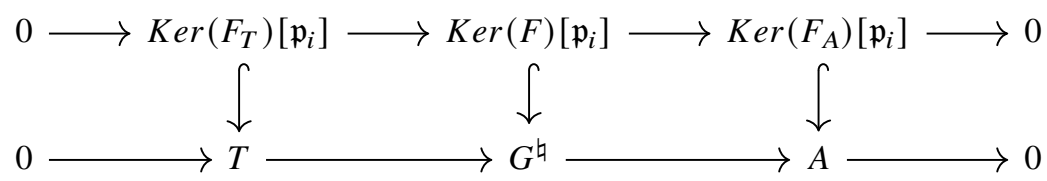

gives us a short exact sequence

$$
0 \rightarrow T^{\left(\mathfrak{p}_{i}\right)} \rightarrow G^{\natural,\left(\mathfrak{p}_{i}\right)} \rightarrow A^{\left(\mathfrak{p}_{i}\right)} \rightarrow 0
$$

Let $m$ be the maximal ideal of $V, k \in \mathbb{Z}, G_{k}^{\natural}:=G_{\operatorname{Spec}\left(V / m^{k}\right)}^{\natural}$ and similarly for other groups defined over $V$. Then the naturality of $\operatorname{Ker}(F)\left[\mathfrak{p}_{i}\right]$ (it commutes with base change) provides isomorphisms

$$
\left(G^{\natural,\left(\mathfrak{p}_{i}\right)}\right)_{k} \cong G_{k}^{\natural} /\left(\operatorname{Ker}(F)\left[\mathfrak{p}_{i}\right]\right) \cong G_{k} /\left(\operatorname{Ker}(F)\left[\mathfrak{p}_{i}\right]\right)=G_{k}^{\left(\mathfrak{p}_{i}\right)},
$$

which are compatible when $k$ varies. This implies that

$$
G_{\text {for }}^{\natural,\left(\mathfrak{p}_{i}\right)} \cong G_{\text {for }}^{\left(\mathfrak{p}_{i}\right)}
$$

hence, we have a canonical isomorphism

$$
G^{\natural,\left(\mathfrak{p}_{i}\right)} \cong G^{\left(\mathfrak{p}_{i}\right), \natural}
$$

by the characterisation of the Raynaud extension.

Now by definition of $X^{\prime}$ and $A^{\prime}$ in the degeneration data, together with the fact $G^{\left(\mathfrak{p}_{i}\right), \natural} \cong G^{\natural,\left(\mathfrak{p}_{i}\right)}$ and

$$
0 \rightarrow T^{\left(\mathfrak{p}_{i}\right)} \rightarrow G^{\natural,\left(\mathfrak{p}_{i}\right)} \rightarrow A^{\left(\mathfrak{p}_{i}\right)} \rightarrow 0
$$

that we have just proved, we have

$$
\begin{gathered}
A^{\prime}=A^{\left(\mathfrak{p}_{i}\right)} \\
X^{\prime}=\operatorname{Hom}\left(T^{\left(\mathfrak{p}_{i}\right)}, \mathbf{G}_{m}\right) \cong X \otimes_{\mathcal{O}_{F}} \mathfrak{p}_{i},
\end{gathered}
$$

where the last isomorphism follows because on a torus we have $F=p$, so

$$
\operatorname{Ker}(F)\left[\mathfrak{p}_{i}\right]=T\left[\mathfrak{p}_{i}\right]=\operatorname{Ker}\left(T=T \otimes_{\mathcal{O}_{F}} \mathcal{O}_{F} \stackrel{i d \otimes \hookrightarrow}{\longrightarrow} T \otimes_{\mathcal{O}_{F}} \mathfrak{p}_{i}^{-1}\right),
$$

which implies that

$$
T^{\left(\mathfrak{p}_{i}\right)} \cong T \otimes_{\mathcal{O}_{F}} \mathfrak{p}_{i}^{-1}
$$

whence the isomorphism on the characters. Note that everything has an $\mathcal{O}$-action, and the isomorphisms are $\mathcal{O}$-equivariant, and in particular the $\mathcal{O}$-structure $i_{A^{\prime}}^{\prime}$ on $A^{\prime}$ is induced from $A$ by the projection $A \rightarrow A^{\left(\mathfrak{p}_{i}\right)}$, which is consistent with partial Frobenius operation on $A$. 
On the other hand, we have a canonical isomorphism

$$
G_{\eta}^{\left(\mathfrak{p}_{i}\right), \vee} \cong G_{\eta}^{\vee,\left(\mathfrak{p}_{i}\right)} \otimes_{\mathcal{O}_{F}} \mathfrak{p}_{i}
$$

as proved in Lemma 3.15, which extends to

$$
G^{\left(\mathfrak{p}_{i}\right), \vee} \cong G^{\vee,\left(\mathfrak{p}_{i}\right)} \otimes_{\mathcal{O}_{F}} \mathfrak{p}_{i}
$$

by formal nonsense (the restriction to the generic fibre is a fully faithful functor from the category of degenerating abelian varieties to that of abelian varieties). We can now take the Raynaud extension of both sides and obtain

$$
G^{\left(\mathfrak{p}_{i}\right), \vee, \natural} \cong G^{\vee,\left(\mathfrak{p}_{i}\right), \natural} \otimes_{\mathcal{O}_{F}} \mathfrak{p}_{i} \cong G^{\vee, \natural,\left(\mathfrak{p}_{i}\right)} \otimes_{\mathcal{O}_{F}} \mathfrak{p}_{i}
$$

where the first isomorphism follows from the functoriality of Raynaud extensions (which implies that $(-) \otimes_{\mathcal{O}_{F}} \mathfrak{p}_{i}$ commutes with the Raynaud extension), and the second isomorphism is the claim we have just proved. From the extension

$$
0 \rightarrow T^{\vee} \rightarrow G^{\vee, \natural} \rightarrow A^{\vee} \rightarrow 0
$$

and the above isomorphism, we see that

$$
0 \rightarrow T^{\vee,\left(\mathfrak{p}_{i}\right)} \otimes_{\mathcal{O}_{F}} \mathfrak{p}_{i} \rightarrow G^{\left(\mathfrak{p}_{i}\right), \vee, \natural} \rightarrow A^{\vee,\left(\mathfrak{p}_{i}\right)} \otimes_{\mathcal{O}_{F}} \mathfrak{p}_{i} \rightarrow 0
$$

We have already observed that from (35) and Lemma 3.15 there are natural isomorphisms $T^{\vee},\left(\mathfrak{p}_{i}\right) \cong$ $T^{\vee} \otimes_{\mathcal{O}_{F}} \mathfrak{p}_{i}^{-1}$ and $A^{\left(\mathfrak{p}_{i}\right), \vee} \cong A^{\vee},\left(\mathfrak{p}_{i}\right) \otimes_{\mathcal{O}_{F}} \mathfrak{p}_{i}$, which simplifies the extension to

$$
0 \rightarrow T^{\vee} \rightarrow G^{\left(\mathfrak{p}_{i}\right), \vee, \natural} \rightarrow A^{\left(\mathfrak{p}_{i}\right), \vee} \rightarrow 0 .
$$

This tells us that the torus part of the dual Raynaud extension of $G^{\prime}$ is the same as that of $G$; hence,

$$
Y^{\prime}=Y
$$

as we expected.

Now we look at the polarisation $\lambda^{\prime}$ and the associated part of the degeneration data. Recall that $\lambda_{\eta}^{\prime}: G_{\eta}^{\prime} \rightarrow G_{\eta}^{\prime \vee}$ is characterised by the formula

$$
\xi \lambda_{\eta}=\pi_{\eta}^{\vee} \circ \lambda_{\eta}^{\prime} \circ \pi_{\eta}
$$

with $\pi_{\eta}: G_{\eta} \rightarrow G_{\eta}^{\prime}$ the projection, which extends uniquely to a morphism $\lambda^{\prime}: G^{\prime} \rightarrow G^{\prime \vee}$ by formal properties of the degenerating abelian varieties. This extension also satisfies the characterising relation

$$
\xi \lambda=\pi^{\vee} \circ \lambda^{\prime} \circ \pi
$$

with $\pi: G \rightarrow G^{\prime}$ the projection (note that $\pi^{\vee}$ here has to be interpreted as the unique extension of the $\pi_{\eta}^{\vee}$ being the dual morphism on the dual abelian varieties). The functoriality of Raynaud extensions provides us with the morphism $\lambda^{\prime \natural}: G^{\prime \natural} \rightarrow G^{\prime \vee \downarrow}$, which fits in a commutative diagram (with change of notation)

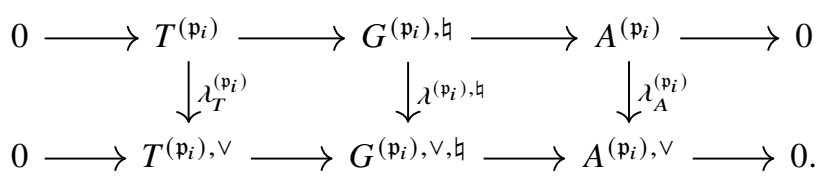


The characterising relation $\xi \lambda=\pi^{\vee} \circ \lambda^{\left(\mathfrak{p}_{i}\right)} \circ \pi$ extends to

$$
\xi \lambda^{\natural}=\pi^{\vee, \natural} \circ \lambda^{\left(\mathfrak{p}_{i}\right), \natural} \circ \pi^{\natural}
$$

on the Raynaud extension by functoriality, which implies the two relations

$$
\begin{aligned}
& \xi \lambda_{A}=\pi_{A}^{\vee, \natural} \circ \lambda_{A}^{\left(\mathfrak{p}_{i}\right)} \circ \pi_{A}^{\natural} \\
& \xi \lambda_{T}=\pi_{T}^{\vee, \natural} \circ \lambda_{T}^{\left(\mathfrak{p}_{i}\right)} \circ \pi_{T}^{\natural}
\end{aligned}
$$

on the abelian and torus parts, respectively. Note that the relation on the abelian part is exactly the characterising relation of the partial Frobenius operation on $\left(A, \lambda_{A}\right)$.

Alternatively, we can write down directly the diagram defining $\lambda^{\prime}$ on the generic fibre, which extends formally to the whole base as follows:

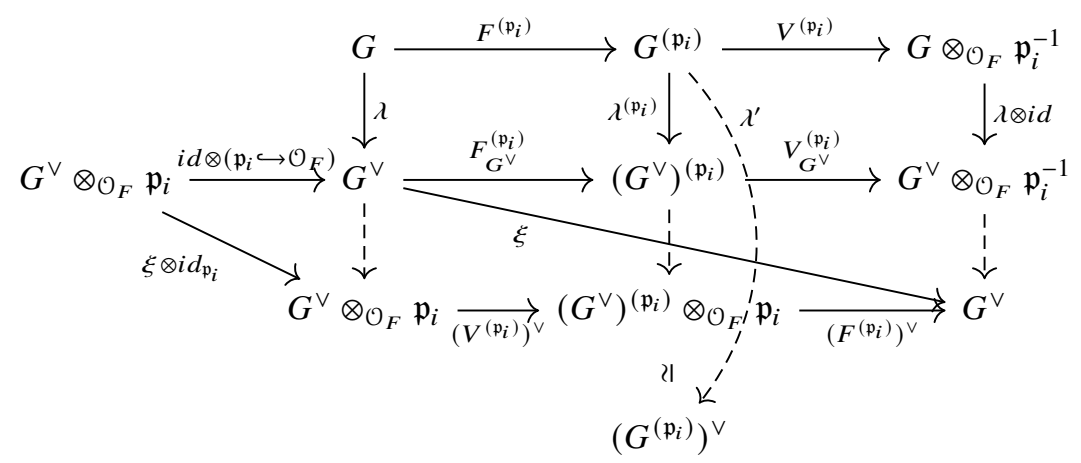

The functoriality of Raynaud extension allows us to draw the same diagram with Raynaud extensions, and so do the abelian and torus parts, with which we obtain a rather explicit description of $\lambda_{A}^{\left(\mathfrak{p}_{i}\right)}$ and $\lambda_{T}^{\left(\mathfrak{p}_{i}\right)}$. This tells us that $\lambda_{A}^{\left(\mathfrak{p}_{i}\right)}$ is obtained as in the partial Frobenius operation, and the morphism $\phi^{\prime}$ on characters induced by $\lambda_{T}^{\left(\mathfrak{p}_{i}\right)}$ is as in the description of the proposition.

The next step is to look at the level structures. Recall that the level structure $\alpha_{n}^{\prime}: L / n L \cong G^{\prime}[n]$ on $G^{\prime}$ is defined by the composition

$$
\alpha_{n}^{\prime}: L / n L \stackrel{\alpha_{n}}{=} G[n] \stackrel{\pi}{\cong} G^{\prime}[n]
$$

where $\pi: G \rightarrow G^{\prime}$ is the projection, which induces an isomorphism on $n$-torsion points because $n$ is prime to $p$. Because $\pi$ preserves the monodromy filtration on $G[n]$ and $G^{\prime}[n]$, we have

$$
Z_{n}^{\prime}=Z_{n}
$$

by definition. More explicitly, $\pi$ induces isomorphisms of the extensions

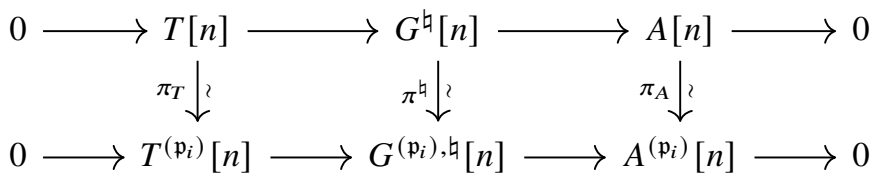




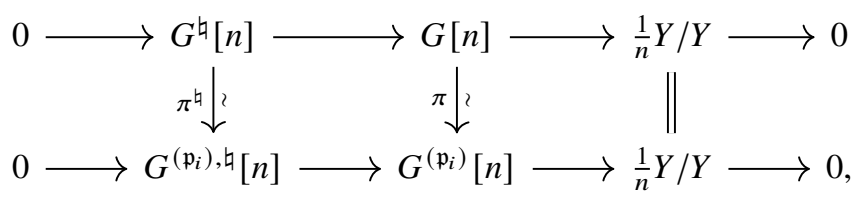

where we use $Y^{\prime}=Y$ in the last isomorphism. We have seen that $\pi_{T}: T \rightarrow T^{\left(\mathfrak{p}_{i}\right)}$ is the natural morphism $T=T \otimes_{\mathcal{O}_{F}} \mathcal{O}_{F} \stackrel{i d \otimes \hookrightarrow}{\longrightarrow} T \otimes_{\mathcal{O}_{F}} \mathfrak{p}_{i}^{-1} \cong T^{\left(\mathfrak{p}_{i}\right)}$, and the corresponding map on characters is $X \otimes_{\mathcal{O}_{F}} \mathfrak{p}_{i} \stackrel{i d \otimes \hookrightarrow}{\longrightarrow} X \otimes_{\mathcal{O}_{F}} \mathcal{O}_{F}=X$, which clearly implies that the degree-2 part of the level- $n$ structure is

$$
\begin{aligned}
& \varphi_{-2, n}^{\prime}: G r_{-2}^{Z_{n}^{\prime}}=G r_{-2}^{Z_{n}} \stackrel{\varphi_{-2, n}}{\longrightarrow} \operatorname{Hom}(X / n X,(\mathbb{Z} / n \mathbb{Z})(1)) \\
& \stackrel{\sim}{\longrightarrow} \operatorname{Hom}\left(X \otimes \mathfrak{p}_{i} / n\left(X \otimes \mathfrak{p}_{i}\right),(\mathbb{Z} / n \mathbb{Z})(1)\right) .
\end{aligned}
$$

Similarly, we have

$$
\varphi_{0, n}^{\prime}: G r_{0}^{Z_{n}^{\prime}}=G r_{0}^{Z_{n}} \stackrel{\varphi_{0, n}}{\longrightarrow} Y / n Y
$$

and

$$
\varphi_{-1, n}^{\prime}: G r_{-1}^{Z_{n}^{\prime}}=G r_{-1}^{Z_{n}} \stackrel{\varphi_{-1, n}}{\longrightarrow} A[n] \stackrel{\pi_{A}}{=} A^{\left(\mathfrak{p}_{i}\right)}[n]
$$

Moreover, we have

$$
v_{-1, n}^{\prime}=v_{n}^{\prime}=v_{n} \circ \kappa,
$$

where the first equality is tautological and the second is part of the definition of the partial Frobenius.

To summarise, we have proved that

$$
\left(A^{\prime}, \lambda_{A^{\prime}}^{\prime}, i_{A^{\prime}}^{\prime},\left(\varphi_{-1, n}^{\prime}, v_{-1, n}^{\prime}\right)\right)
$$

is exactly the partial Frobenius operation applied to $\left(A, \lambda_{A}, i_{A},\left(\varphi_{-1, n}, v_{-1, n}\right)\right)$ and have identified the torus argument

$$
\left(X^{\prime}, Y^{\prime}, \phi^{\prime}, \varphi_{-2, n}^{\prime}, \varphi_{0, n}^{\prime}\right)
$$

together with the filtration $Z_{n}^{\prime}$. The remaining part to be identified is $\left(c^{\prime}, c^{\prime \vee}, \tau^{\prime}\right)$.

First, we note that for any degenerating abelian variety $H$, the canonical morphism $H=H \otimes_{\mathcal{O}_{F}}$ $\mathcal{O}_{F} \stackrel{i d \otimes \hookrightarrow}{\longrightarrow} H \otimes_{\mathcal{O}_{F}} \mathfrak{p}_{i}^{-1}$ factors through $H \rightarrow H^{\left(\mathfrak{p}_{i}\right)} \rightarrow H \otimes_{\mathcal{O}_{F}} \mathfrak{p}_{i}^{-1}$, and tensoring the factoring morphism with $\mathfrak{p}_{i}$ we obtain

$$
V^{\left(\mathfrak{p}_{i}\right)}: H^{\left(\mathfrak{p}_{i}\right)} \otimes_{\mathcal{O}_{F}} \mathfrak{p}_{i} \rightarrow H
$$

which is characterised by the commutative diagram

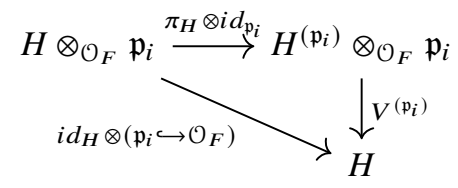

We observe that when $H$ is an abelian scheme, under the identification $H^{\left(\mathfrak{p}_{i}\right), \vee} \cong H^{\vee},\left(\mathfrak{p}_{i}\right) \otimes_{\mathcal{O}_{F}} \mathfrak{p}_{i}$ the morphism $H^{\left(\mathfrak{p}_{i}\right)} \otimes_{\mathcal{O}_{F}} \mathfrak{p}_{i} \rightarrow H$ is the dual of the projection $\pi_{H}: H \rightarrow H^{\left(\mathfrak{p}_{i}\right)}$; that is, we have a 
commutative diagram

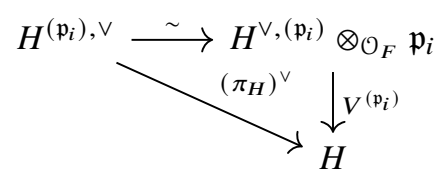

Dually, we have that $V^{\left(\mathfrak{p}_{i}\right), \vee}$ is $\pi_{A^{\vee}}$ under canonical isomorphism; that is,

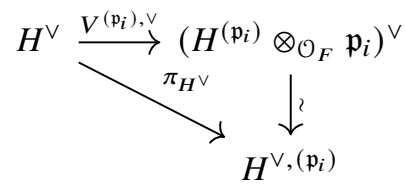

is commutative.

\section{Lemma 5.24.}

$$
c^{\prime}: X^{\prime}=X \otimes_{\mathcal{O}_{F}} \mathfrak{p}_{i} \stackrel{c \otimes i d}{\longrightarrow} A^{\vee} \otimes_{\mathcal{O}_{F}} \mathfrak{p}_{i} \stackrel{\pi \otimes i d}{\longrightarrow} A^{\vee \prime} \otimes_{\mathcal{O}_{F}} \mathfrak{p}_{i} \cong A^{\prime \vee} .
$$

Proof of the Lemma. We have seen that there is a natural morphism

$$
V^{\left(\mathfrak{p}_{i}\right)}: G^{\natural,\left(\mathfrak{p}_{i}\right)} \otimes_{\mathcal{O}_{F}} \mathfrak{p}_{i} \rightarrow G^{\natural}
$$

that induces the morphism between extensions

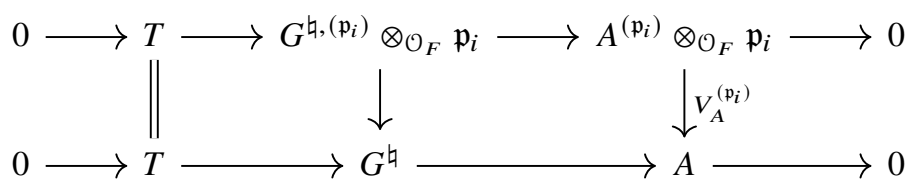

where we use the canonical isomorphism $T^{\left(\mathfrak{p}_{i}\right)} \otimes_{\mathcal{O}_{F}} \mathfrak{p}_{i} \cong T$. We note that the extension in the first row is determined by the morphism

$$
X \cong\left(X \otimes_{\mathcal{O}_{F}} \mathfrak{p}_{i}\right) \otimes_{\mathcal{O}_{F}} \mathfrak{p}_{i}^{-1} \stackrel{c^{\prime} \otimes i d_{\mathfrak{p}_{i}^{-1}}}{\longrightarrow} A^{\left(\mathfrak{p}_{i}\right), \vee} \otimes_{\mathcal{O}_{F}} \mathfrak{p}_{i}^{-1} \cong A^{\vee,\left(\mathfrak{p}_{i}\right)}
$$

and the extension in the second row is determined by

$$
c: X \longrightarrow A^{\vee}
$$

For $\chi \in X$, we write $\mathcal{O}_{\chi}:=c(\chi) \in \operatorname{Pic}^{0}(A)$ and $L_{\chi}:=c^{\prime} \otimes i d_{\mathfrak{p}_{i}^{-1}}(\chi) \in \operatorname{Pic}^{0}\left(A^{\left(\mathfrak{p}_{i}\right)} \otimes_{\mathcal{O}_{F}} \mathfrak{p}_{i}\right)$. By abuse of notation, we will write $\mathscr{O}_{\chi}$ and $L_{\chi}$ for both the line bundle and the $\mathbf{G}_{m}$-torsor.

Recall that $\mathcal{O}_{\chi}$ is defined as the pushout of $G^{\natural}$ along $\chi$; that is, we have a pushout diagram

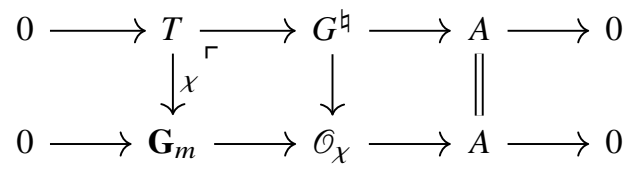


and similarly for $L_{\chi}$. We can complete this in the previous diagram and obtain

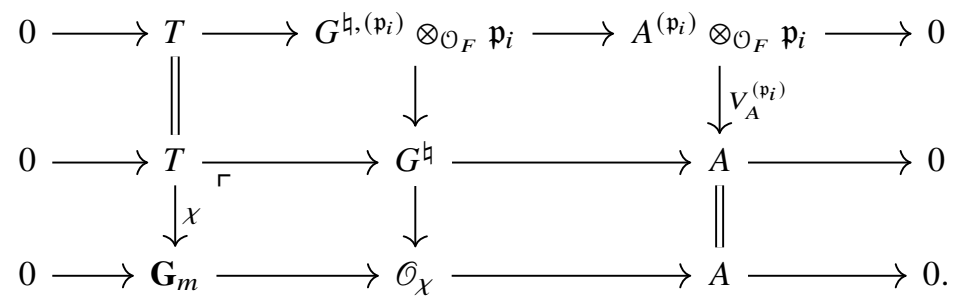

Similarly, $L_{\chi}$ is defined by the pushout of $G^{\natural,\left(\mathfrak{p}_{i}\right)} \otimes_{\mathcal{O}_{F}} \mathfrak{p}_{i}$ along $\chi$, and the universal property of pushout implies that the diagram factorises through $L_{\chi}$; that is, we have a commutative diagram

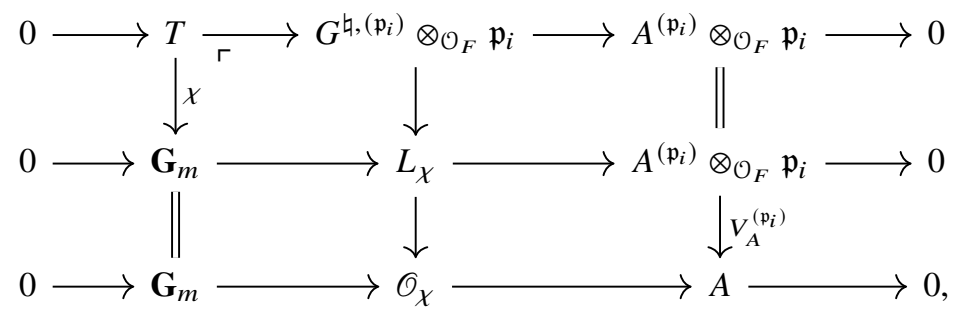

which tells us that

$$
\left(V_{A}^{\left(\mathfrak{p}_{i}\right)}\right)^{*} \mathcal{O}_{\chi}=L_{\chi}
$$

In other words, we have a commutative diagram

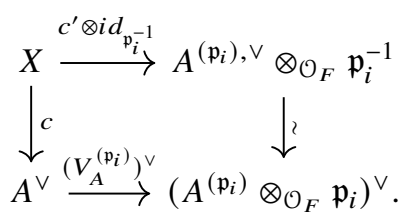

Now with the help of the commutative diagram

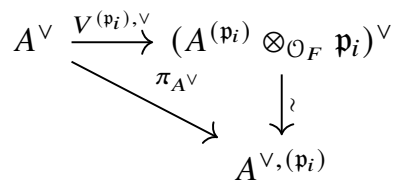

we see that

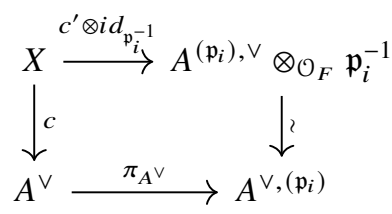

that is, under the identification $A^{\vee,\left(\mathfrak{p}_{i}\right)} \cong A^{\left(\mathfrak{p}_{i}\right), \vee} \otimes_{\mathcal{O}_{F}} \mathfrak{p}_{i}^{-1}$,

$$
c^{\prime} \otimes i d_{\mathfrak{p}_{i}^{-1}}=\pi_{A^{\vee}} \circ c
$$


and tensoring with $\mathfrak{p}_{i}$ we obtain

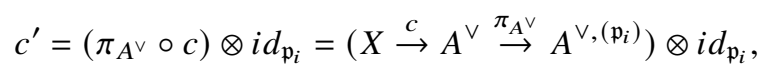

which is what we want to prove.

Dually, we have the following.

\section{Lemma 5.25.}

$$
c^{\prime \vee}: Y^{\prime}=Y \stackrel{c^{\vee}}{\longrightarrow} A \stackrel{\pi}{\longrightarrow} A^{\prime} .
$$

Proof of the Lemma. For $y \in Y$, we write $L_{c^{\vee}(y)}:=c^{\vee}(y) \in \operatorname{Pic}^{0}\left(A^{\vee}\right)$ and $L_{c^{\prime \vee}(y)}:=c^{\prime \vee}(y) \in$ $\operatorname{Pic}{ }^{0}\left(A^{\left(\mathfrak{p}_{i}\right), \vee}\right)$. We have seen that $G^{\left(\mathfrak{p}_{i}\right), \vee, \natural} \cong\left(G^{\vee, \natural}\right)^{\left(\mathfrak{p}_{i}\right)} \otimes_{\mathcal{O}_{F}} \mathfrak{p}_{i}$, which equips with a natural morphism

$$
\left(G^{\vee, \natural}\right)^{\left(\mathfrak{p}_{i}\right)} \otimes_{\mathcal{O}_{F}} \mathfrak{p}_{i} \rightarrow G^{\vee, \natural}
$$

Recall that $G^{\left(\mathfrak{p}_{i}\right), \vee, \natural}$ is an extension

$$
0 \rightarrow T^{\vee} \rightarrow G^{\left(\mathfrak{p}_{i}\right), \vee, \natural} \rightarrow A^{\left(\mathfrak{p}_{i}\right), \vee} \rightarrow 0
$$

and we observe that the morphism $G^{\left(\mathfrak{p}_{i}\right), \vee, \natural} \cong\left(G^{\vee, \natural}\right)^{\left(\mathfrak{p}_{i}\right)} \otimes_{\mathcal{O}_{F}} \mathfrak{p}_{i} \rightarrow G^{\vee, \natural}$ gives rise to a morphism of the extension

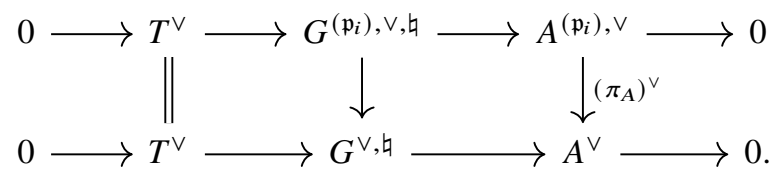

Now, similar to before, $L_{c^{\vee}(y)}$ is the pushout of $G^{\vee, \natural}$ along $y$, and we have a commutative diagram

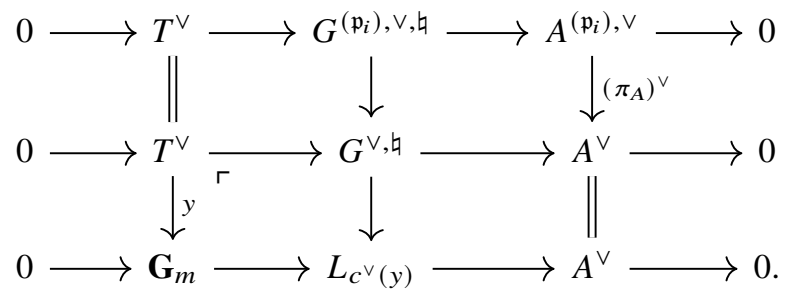

By the universal property of pushout, we have the factorisation

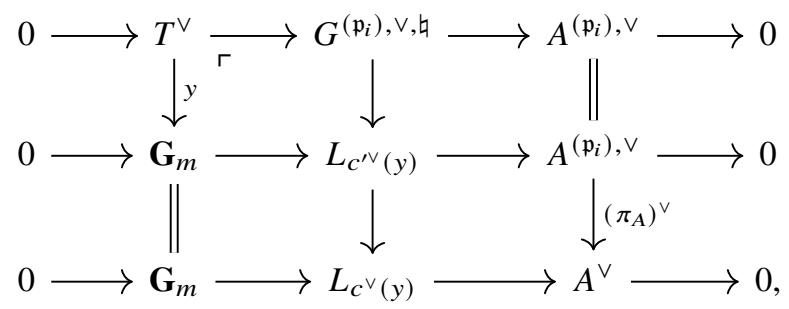

which implies that

$$
\left(\pi_{A}\right)^{\vee *} L_{c^{\vee}(y)}=L_{c^{\prime}}(y)
$$

In other words, we have

$$
c^{\prime \vee}(y)=\left(\left(\pi_{A}\right)^{\vee}\right)^{\vee} \circ c^{\vee}(y)=\pi_{A} \circ c^{\vee}(y)
$$


under the canonical identification $\left(A^{\left(\mathfrak{p}_{i}\right), \vee}\right)^{\vee}=A^{\left(\mathfrak{p}_{i}\right)}$, which means that

$$
c^{\prime \vee}: Y \stackrel{c^{\vee}}{\longrightarrow} A \stackrel{\pi_{A}}{\longrightarrow} A^{\left(\mathfrak{p}_{i}\right)} .
$$

Lastly, we determine $\tau^{\prime}$ from $\tau$.

Let us write $\mathcal{O}_{\chi}:=c(\chi) \in \operatorname{Pic} c^{0}(A)$ for $\chi \in X$, and similarly $\mathscr{O}_{\chi^{\prime}}:=c^{\prime}\left(\chi^{\prime}\right) \in \operatorname{Pic}^{0}\left(A^{\prime}\right)$ for $\chi^{\prime} \in X^{\prime}$. By abuse of notation, we will write $\mathcal{O}_{\chi}$ for both the line bundle and the $\mathbf{G}_{m}$-torsor.

We first make an observation on the relation between $\mathcal{O}_{\chi}$ and $\mathcal{O}_{\chi^{\prime}}$, which can be used to write the canonical morphism $G^{\natural} \rightarrow G^{\left(\mathfrak{p}_{i}\right), \natural}$ in a more explicit way.

Recall that $\mathcal{O}_{\chi}$ is defined as the pushout of $G^{\natural}$ along $\chi$; that is, we have a pushout diagram

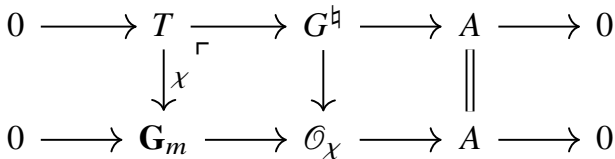

and similarly for $\mathcal{O}_{\chi^{\prime}}$. We have a diagram

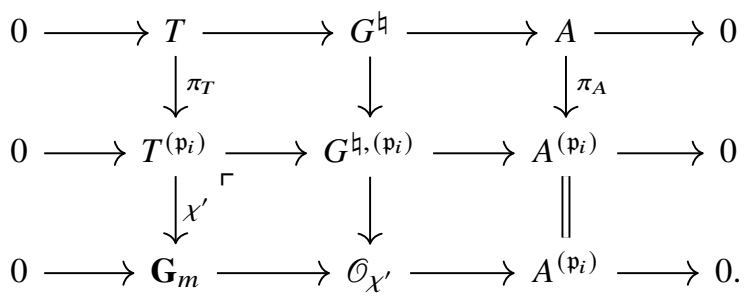

Let

$$
\rho: X^{\prime}=X \otimes_{\mathcal{O}_{F}} \mathfrak{p}_{i} \rightarrow X
$$

be the map induced by $\pi_{T}$ (being the obvious map induced by $\mathfrak{p}_{i} \hookrightarrow \mathcal{O}_{F}$ ), then $\mathcal{O}_{\rho}\left(\chi^{\prime}\right)$ is the pushout along $\pi_{T} \circ \chi^{\prime}$, and the universal property of the pushout provides us with a factorisation of the short exact sequence

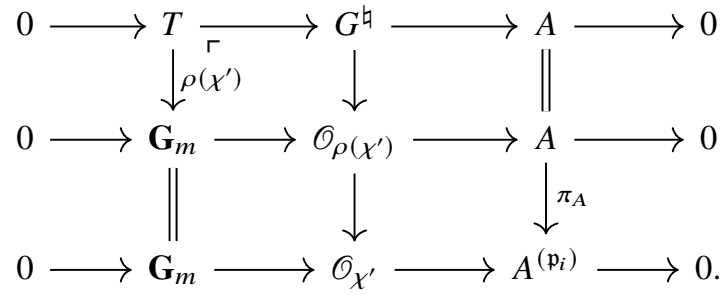

This shows

$$
\pi_{A}^{*} \mathcal{O}_{\chi^{\prime}}=\mathcal{O}_{\rho\left(\chi^{\prime}\right)},
$$

which implies that under the identifications $G^{\natural}=\underline{\operatorname{Spec}} \mathscr{O}_{A}\left(\underset{\chi \in X}{\oplus} \mathscr{O}_{\chi}\right)$ and $G^{\left(\mathfrak{p}_{i}\right), \natural}=\underline{\operatorname{Spec}} \mathscr{O}_{\left.A^{\left(p_{i}\right.}\right)}\left(\underset{\chi^{\prime} \in X^{\prime}}{\oplus} \mathcal{O}_{\chi^{\prime}}\right)$ the morphism

$$
G^{\natural} \rightarrow G^{\left(\mathfrak{p}_{i}\right), \natural}
$$


is induced from the map

$$
\pi_{A}^{*}\left(\underset{\chi^{\prime} \in X^{\prime}}{\oplus} \mathcal{O}_{\chi^{\prime}}\right) \cong \underset{\chi^{\prime} \in X^{\prime}}{\oplus} \pi_{A}^{*} \mathcal{O}_{\chi^{\prime}} \cong \underset{\chi^{\prime} \in X^{\prime}}{\oplus} \mathcal{O}_{\rho\left(\chi^{\prime}\right)} \hookrightarrow \underset{\chi \in X}{\oplus} \mathcal{O}_{\chi}
$$

on relatively affine algebras over $A$.

Let us recall how we associate $\tau$ to the degenerating abelian variety $G$. We start by choosing an ample invertible cubical sheaf $\mathcal{L}$ on $G$ (whose existence is guaranteed by the normality of the base $\operatorname{Spev}(V)$ ), then we can show that its formal completion extends canonically to a cubical ample line bundle $\mathcal{L}^{\natural}$ on $G^{\natural}$, which descends to an ample invertible sheaf $\mathcal{M}$ on $A$; that is, if we denote by $p: G^{\natural} \rightarrow A$ the projection map, then $p^{*} \mathcal{M}=\mathcal{L}^{\natural}$. Replacing $\lambda_{\eta}$ by $\lambda_{\mathcal{L}_{\eta}}$ if necessary (so $\lambda$ is the unique extension of $\lambda_{\mathcal{L}_{\eta}}$ to $G$ ), we assume that $\lambda_{\eta}=\lambda_{\mathcal{L}_{\eta}}$. The construction of $\tau$ is independent of the choice of $\lambda$ or $\mathcal{L}$.

The canonical isomorphism $G^{\natural}=\underline{\operatorname{Spec}} \mathscr{O}_{A}\left(\underset{\chi \in X}{\oplus} \mathcal{O}_{\chi}\right)$ tells us that

$$
p_{*} \mathcal{L}^{\natural} \cong \underset{\chi \in X}{\oplus} \mathcal{M}_{\chi}
$$

with $\mathcal{M}_{\chi}:=\mathcal{M} \otimes_{\mathcal{O}_{A}} \mathcal{O}_{\chi}$, from which we obtain

$$
\Gamma\left(G^{\natural}, \mathcal{L}^{\natural}\right)=\underset{\chi \in X}{\oplus} \Gamma\left(A, \mathcal{M}_{\chi}\right)
$$

and

$$
\Gamma\left(G_{\text {for }}^{\natural}, \mathcal{L}_{\text {for }}^{\natural}\right)=\underset{\chi \in X}{\hat{\oplus}} \Gamma\left(A, \mathcal{M}_{\chi}\right)
$$

where^denotes the completion with respect to the maximal ideal of $V$. Now we have the canonical map

$$
\Gamma(G, \mathcal{L}) \rightarrow \Gamma\left(G_{\text {for }}, \mathcal{L}_{\text {for }}\right) \cong \Gamma\left(G_{\text {for }}^{\natural}, \mathcal{L}_{\text {for }}^{\natural}\right) \cong \underset{\chi \in X}{\hat{\oplus}} \Gamma\left(A, \mathcal{M}_{\chi}\right) \rightarrow \Gamma\left(A, \mathcal{M}_{\chi}\right)
$$

where the first map is the restriction and the last is the projection on the $\chi$ th component. Tensoring both sides with $\operatorname{Frac}(V)$, we obtain

$$
\sigma_{\chi}: \Gamma\left(G_{\eta}, \mathcal{L}_{\eta}\right) \longrightarrow \Gamma\left(A_{\eta}, \mathcal{M}_{\chi, \eta}\right)
$$

Let $y \in Y$ and $T_{c^{\vee}(y)}: A \rightarrow A$ the translation by $c^{\vee}(y)$, then

$$
\lambda_{A} \circ c^{\vee}=c \circ \phi
$$

tells us that we have a canonical isomorphism of rigidified line bundles

$$
T_{c^{\vee}(y)}^{*} \mathcal{M}_{\chi} \cong \mathcal{M}_{\chi+\phi(y)} \otimes_{R} \mathcal{M}_{\chi}\left(c^{\vee}(y)\right),
$$

and this is the place where we use the assumption $\lambda_{\eta}=\lambda_{\mathcal{L}_{\eta}}$ on $\mathcal{L}$. Now we have the map

$$
T_{c^{\vee}(y)}^{*} \circ \sigma_{\chi}: \Gamma\left(G_{\eta}, \mathcal{L}_{\eta}\right) \rightarrow \Gamma\left(A_{\eta}, T_{c^{\vee}(y)}^{*} \mathcal{M}_{\chi, \eta}\right) \cong \Gamma\left(A_{\eta}, \mathcal{M}_{\chi+\phi(y), \eta}\right) \otimes_{K} \mathcal{M}_{\chi}\left(c^{\vee}(y)\right)_{\eta} .
$$

The desired $\tau$ is obtained by comparing $T_{c^{\vee}(y)}^{*} \circ \sigma_{\chi}$ with the map

$$
\sigma_{\chi+\phi(y)}: \Gamma\left(G_{\eta}, \mathcal{L}_{\eta}\right) \longrightarrow \Gamma\left(A_{\eta}, \mathcal{M}_{\chi+\phi(y), \eta}\right)
$$

and the result is

$$
\sigma_{\chi+\phi(y)}=\psi(y) \tau(y, \chi) T_{c^{\vee}(y)}^{*} \circ \sigma_{\chi},
$$


where

$$
\psi(y): \mathcal{M}\left(c^{\vee}(y)\right)_{\eta} \stackrel{\sim}{\rightarrow} \mathcal{O}_{S, \eta}
$$

is a trivialisation of the fibre of $\mathcal{M}$ at $c^{\vee}(y)$ and

$$
\tau(y, \chi): \mathcal{O}_{\chi}\left(c^{\vee}(y)\right)_{\eta} \longrightarrow \mathcal{O}_{S, \eta}
$$

is a section of $\mathcal{O}_{\chi}\left(c^{\vee}(y)\right)_{\eta}^{\otimes-1}$ for each $y \in Y$ and $\chi \in X$, so that $\psi(y) \tau(y, \chi)$ is a section of $\mathcal{M}_{\chi}\left(c^{\vee}(y)\right)_{\eta}^{\otimes-1}$ (recall $\mathcal{M}_{\chi}=\mathcal{M} \otimes \mathcal{O}_{\chi}$ ). This uniquely characterises $\tau$ because $\sigma_{\chi} \neq 0$ for every $\chi \in X$.

Lemma 5.26. There is a canonical isomorphism

$$
\left(c^{\vee \vee} \times c^{\prime}\right)^{*} P_{A^{\prime}} \cong\left(\left(c^{\vee} \times c\right)^{*} P_{A}\right)_{Y^{\prime} \times X^{\prime}}
$$

where the pullback to $Y^{\prime} \times X^{\prime}$ is through the natural injection

$$
Y^{\prime} \times X^{\prime}=Y \times\left(X \otimes_{\mathcal{O}_{F}} \mathfrak{p}_{i}\right) \hookrightarrow Y \times X
$$

induced by $X \otimes_{\mathcal{O}_{F}} \mathfrak{p}_{i} \hookrightarrow X \otimes_{\mathcal{O}_{F}} \mathcal{O}_{F} \cong X$. Now $\tau^{\prime}$ is identified as

$$
\tau^{\prime}: 1_{Y^{\prime} \times X^{\prime}, \eta} \stackrel{\tau}{\stackrel{\left.\tau\right|_{Y^{\prime} \times X^{\prime}}}{\longrightarrow}}\left(\left(c^{\vee} \times c\right)^{*} \mathcal{P}_{A, \eta}^{\otimes-1}\right)_{Y^{\prime} \times X^{\prime}} \cong\left(c^{\vee} \times c^{\prime}\right)^{*} P_{A^{\prime}, \eta}^{\otimes-1} .
$$

Remark 5.27. As in Subsection 5.1.3, the lemma is equivalent to the statement that the period of $G^{\left(\mathfrak{p}_{i}\right)}$ is given by

$$
Y_{\eta} \stackrel{\iota}{\longrightarrow} G_{\eta}^{\natural} \longrightarrow G_{\eta}^{\left(\mathfrak{p}_{i}\right), \natural},
$$

where $\iota$ is the period of $G$ and $G_{\eta}^{\natural} \longrightarrow G_{\eta}^{\left(\mathfrak{p}_{i}\right), \natural}$ is the natural projection map.

Proof of the Lemma. We have the same description as above for $\tau^{\prime}$, and we want to compare it with $\tau$. Let us first compare $\sigma_{\chi^{\prime}}$ and $\sigma_{\chi}$.

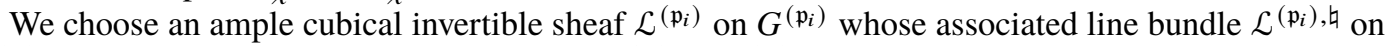
$G^{\left(\mathfrak{p}_{i}\right), \mathfrak{G}}$ descends to an ample invertible sheaf $\mathcal{M}^{\left(\mathfrak{p}_{i}\right)}$ on $A^{\left(\mathfrak{p}_{i}\right)}$. Let $\mathcal{L}$ (respectively $\mathcal{M}$ ) be the pullback of $\mathcal{L}^{\left(\mathfrak{p}_{i}\right)}$ (respectively $\mathcal{M}^{\left(\mathfrak{p}_{i}\right)}$ ) along the natural map $G \rightarrow G^{\left(\mathfrak{p}_{i}\right)}$ (respectively $A \rightarrow A^{\left(\mathfrak{p}_{i}\right)}$ ). Note that both $\mathcal{L}$ and $\mathcal{M}$ are ample because they are pullbacks of ample line bundles along finite maps $G \rightarrow G^{\left(\mathfrak{p}_{i}\right)}$ and $A \rightarrow A^{\left(\mathfrak{p}_{i}\right)}$, respectively.

We assume that $\mathcal{L}_{\eta}^{\left(\mathfrak{p}_{i}\right)}$ induces the polarisation $\lambda_{\eta}^{\prime}$ on $G_{\eta}^{\left(\mathfrak{p}_{i}\right)}$, so we have

$$
T_{c^{\prime \vee}(y)}^{*} \mathcal{M}_{\chi^{\prime}}^{\left(\mathfrak{p}_{i}\right)} \cong \mathcal{M}_{\chi^{\prime}+\phi^{\prime}(y)}^{\left(\mathfrak{p}_{i}\right)} \otimes_{R} \mathcal{M}_{\chi^{\prime}}^{\left(\mathfrak{p}_{i}\right)}\left(c^{\prime \vee}(y)\right)
$$

Let $\pi: G \rightarrow G^{\left(\mathfrak{p}_{i}\right)}$ be the projection map, then $\mathcal{L}:=\pi^{*} \mathcal{L}^{\left(\mathfrak{p}_{i}\right)}$ and the associated polarisation

$$
\lambda_{\mathcal{L}_{\eta}}=\lambda_{\pi^{*} \mathcal{L}_{\eta}^{\left(\mathfrak{p}_{i}\right)}}=\pi_{\eta}^{\vee} \circ \lambda_{\mathcal{L}_{\eta}^{\left(\mathfrak{p}_{i}\right)}} \circ \pi_{\eta}=\pi_{\eta}^{\vee} \circ \lambda_{\eta}^{\prime} \circ \pi_{\eta}=\xi \lambda_{\eta}
$$

which has the effect that

$$
T_{c^{\vee}(y)}^{*} \mathcal{M}_{\chi} \cong \mathcal{M}_{\chi+\xi \phi(y)} \otimes_{R} \mathcal{M}_{\chi}\left(c^{\vee}(y)\right)
$$

because we have to replace the relation $\lambda_{A} \circ c^{\vee}=c \circ \phi$ by $\xi \lambda_{A} \circ c^{\vee}=c \circ \xi \phi$.

Let

$$
\rho: X^{\prime}=X \otimes_{\mathcal{O}_{F}} \mathfrak{p}_{i} \rightarrow X
$$


be the map induced by $\mathfrak{p}_{i} \hookrightarrow \mathcal{O}_{F}$ as before, then for $\chi^{\prime} \in X^{\prime}$, the natural map $G \rightarrow G^{\left(\mathfrak{p}_{i}\right)}$ induces a commutative diagram

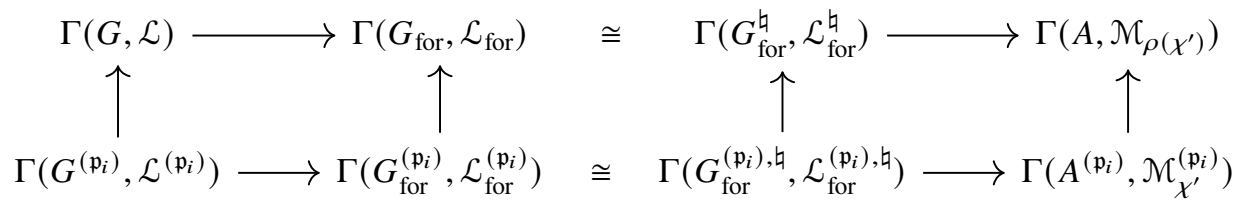

where the last two horizontal maps are projections

$$
\Gamma\left(G_{\text {for }}^{\natural}, \mathcal{L}_{\text {for }}^{\natural}\right) \cong \hat{\oplus}_{\chi \in X} \Gamma\left(A, \mathcal{M}_{\chi}\right) \rightarrow \Gamma\left(A, \mathcal{M}_{\rho\left(\chi^{\prime}\right)}\right)
$$

and

$$
\Gamma\left(G_{\text {for }}^{\left(\mathfrak{p}_{i}\right), \mathfrak{G}}, \mathcal{L}_{\text {for }}^{\left(\mathfrak{p}_{i}\right), \natural}\right) \cong \underset{\chi^{\prime} \in X}{\hat{\oplus}} \Gamma\left(A^{\left(\mathfrak{p}_{i}\right)}, \mathcal{M}_{\chi^{\prime}}^{\left(\mathfrak{p}_{i}\right)}\right) \rightarrow \Gamma\left(A^{\left(\mathfrak{p}_{i}\right)}, \mathcal{M}_{\chi^{\prime}}^{\left(\mathfrak{p}_{i}\right)}\right)
$$

respectively, and the last vertical map is induced from

$$
\pi_{A}^{*} \mathcal{M}_{\chi^{\prime}}^{\left(\mathfrak{p}_{i}\right)}=\pi_{A}^{*}\left(\mathcal{M}^{\left(\mathfrak{p}_{i}\right)} \otimes_{\mathcal{O}_{A}\left(\mathfrak{p}_{i}\right)} \mathcal{O}_{\chi^{\prime}}\right)=\mathcal{M} \otimes_{\mathcal{O}_{A}} \pi^{*} \mathcal{O}_{\chi^{\prime}} \stackrel{(36)}{\cong} \mathcal{M} \otimes_{\mathcal{O}_{A}} \mathcal{O}_{\rho\left(\chi^{\prime}\right)}=\mathcal{M} \rho_{\rho\left(\chi^{\prime}\right)}
$$

that is, it is taking the global section of the map $\mathcal{M}_{\chi^{\prime}}^{\left(\mathfrak{p}_{i}\right)} \rightarrow \pi_{A *} \pi_{A}^{*} \mathcal{M}_{\chi^{\prime}}^{\left(\mathfrak{p}_{i}\right)} \stackrel{\sim}{\rightarrow} \pi_{A *} \mathcal{M}_{\rho\left(\chi^{\prime}\right)}$ with the last isomorphism being $\pi_{A *}$ of the isomorphism (36). The commutativity of the first two squares is tautological, and that of the last square follows from (37). Tensoring with $\operatorname{Frac}(V)$ of the above diagram, we obtain a commutative diagram

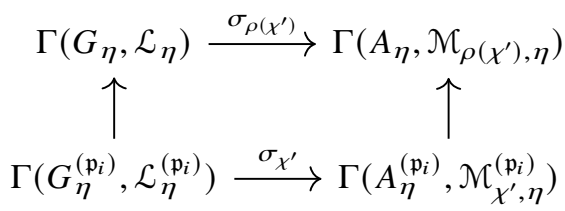

for every $\chi^{\prime} \in X^{\prime}$.

For $y \in Y$, we can complete the diagram as

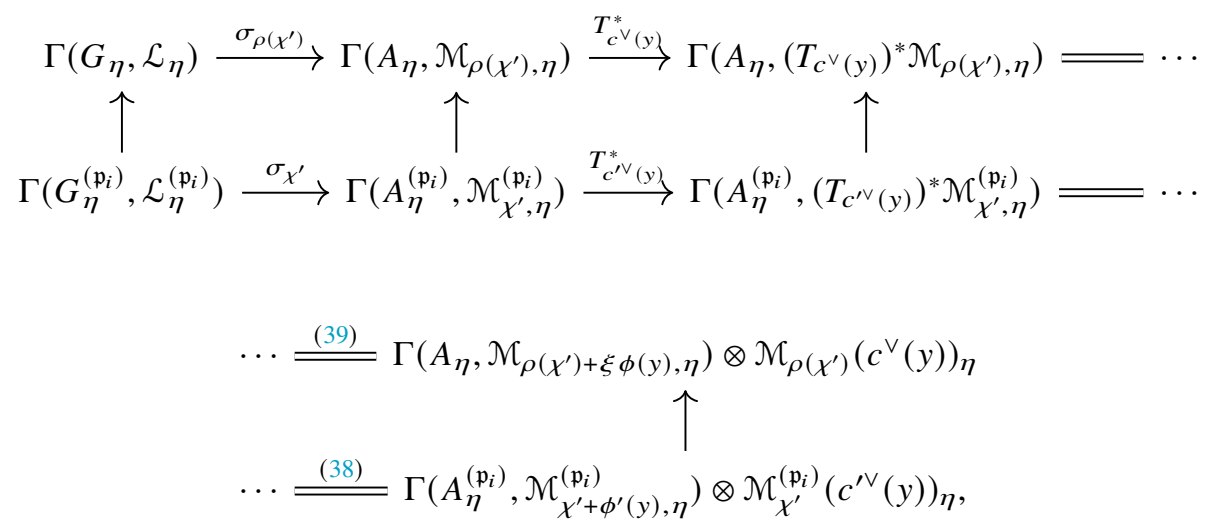


where the last vertical arrow is the tensor product of the morphism

$$
\Gamma\left(A_{\eta}^{\left(\mathfrak{p}_{i}\right)}, \mathcal{M}_{\chi^{\prime}+\phi^{\prime}(y), \eta}^{\left(\mathfrak{p}_{i}\right)}\right) \rightarrow \Gamma\left(A_{\eta}, \mathcal{M}_{\rho\left(\chi^{\prime}\right)+\xi \phi(y), \eta}\right)
$$

induced by $\pi_{A}^{*} \mathcal{M}_{\chi^{\prime}+\phi^{\prime}(y)}^{\left(\mathfrak{p}_{i}\right)} \cong \mathcal{M}_{\rho\left(\chi^{\prime}+\phi^{\prime}(y)\right)}=\mathcal{M}_{\rho\left(\chi^{\prime}\right)+\xi \phi(y)}\left(\rho \circ \phi^{\prime}=\xi \phi\right.$ by the diagram defining $\left.\phi^{\prime}\right)$, with the isomorphism

$$
\mathcal{M}_{\rho\left(\chi^{\prime}\right)}\left(c^{\vee}(y)\right)_{\eta}=\pi_{A}^{*} \mathcal{M}_{\chi^{\prime}}^{\left(\mathfrak{p}_{i}\right)}\left(c^{\vee}(y)\right)_{\eta}=\mathcal{M}_{\chi^{\prime}}^{\left(\mathfrak{p}_{i}\right)}\left(\pi_{A} \circ c^{\vee}(y)\right)_{\eta}=\mathcal{M}_{\chi^{\prime}}^{\left(\mathfrak{p}_{i}\right)}\left(c^{\prime \vee}(y)\right)_{\eta},
$$

where we use $c^{\prime \vee}=\pi_{A} \circ c^{\vee}$ in the last equality. The middle square commutes because we have a commutative diagram

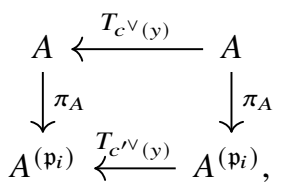

which follows from $c^{\prime \vee}=\pi_{A} \circ c^{\vee}$ and $\pi_{A}$ being a group homomorphism. The commutativity of the last square follows from the commutativity of the square

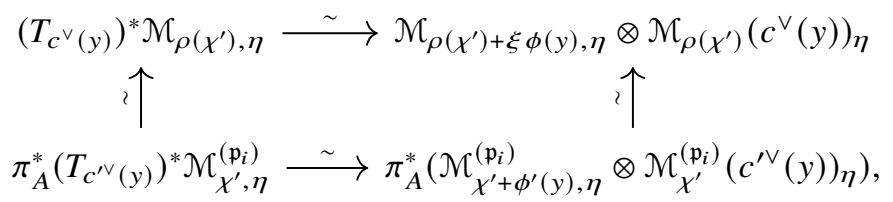

and we can prove its commutativity as follows. Recall that the first horizontal map is (using $\lambda_{L}(x)=$ $\left.T_{x}^{*} L \otimes L^{-1} \otimes L_{x}^{-1}\right)$

$$
\begin{gathered}
\left(T_{c^{\vee}(y)}\right)^{*} \mathcal{M}_{\rho\left(\chi^{\prime}\right), \eta}=\left(\lambda_{\mathcal{M}} \circ c^{\vee}(y)\right)_{\eta} \otimes \mathcal{M}_{\rho\left(\chi^{\prime}\right), \eta} \otimes \mathcal{M}_{\rho\left(\chi^{\prime}\right)}\left(c^{\vee}(y)\right)_{\eta} \\
\stackrel{\lambda_{\mathcal{M}=\xi \lambda_{A}}}{=}(c \circ \xi \phi(y))_{\eta} \otimes \mathcal{M}_{\rho\left(\chi^{\prime}\right), \eta} \otimes \mathcal{M}_{\rho\left(\chi^{\prime}\right)}\left(c^{\vee}(y)\right)_{\eta} \\
=\mathcal{O}_{\xi \phi(y), \eta} \otimes \mathcal{M}_{\rho\left(\chi^{\prime}\right), \eta} \otimes \mathcal{M}_{\rho\left(\chi^{\prime}\right)}\left(c^{\vee}(y)\right)_{\eta} \\
=\mathcal{M}_{\rho\left(\chi^{\prime}\right)+\xi \phi(y), \eta} \otimes \mathcal{M}_{\rho\left(\chi^{\prime}\right)}\left(c^{\vee}(y)\right)_{\eta}
\end{gathered}
$$

and similarly the second horizontal map is $\pi_{A}^{*}$ of

$$
\begin{gathered}
\left(T_{c^{\prime \vee}(y)}\right)^{*} \mathcal{M}_{\chi^{\prime}, \eta}^{\left(\mathfrak{p}_{i}\right)}=\left(\lambda_{A^{\prime}}^{\prime} \circ c^{\prime \vee}(y)\right)_{\eta} \otimes \mathcal{M}_{\chi^{\prime}, \eta}^{\left(\mathfrak{p}_{i}\right)} \otimes \mathcal{M}_{\chi^{\prime}}^{\left(\mathfrak{p}_{i}\right)}\left(c^{\prime \vee}(y)\right)_{\eta} \\
=\left(c^{\prime} \circ \phi^{\prime}(y)\right)_{\eta} \otimes \mathcal{M}_{\chi^{\prime}, \eta}^{\left(\mathfrak{p}_{i}\right)} \otimes \mathcal{M}_{\chi^{\prime}}^{\left(\mathfrak{p}_{i}\right)}\left(c^{\prime \vee}(y)\right)_{\eta} \\
=\sigma_{\phi^{\prime}(y), \eta} \otimes \mathcal{M}_{\chi^{\prime}, \eta}^{\left(\mathfrak{p}_{i}\right)} \otimes \mathcal{M}_{\chi^{\prime}}^{\left(\mathfrak{p}_{i}\right)}\left(c^{\prime \vee}(y)\right)_{\eta} \\
=\mathcal{M}_{\chi^{\prime}+\phi^{\prime}(y), \eta}^{\left(\mathfrak{p}_{i}\right)} \otimes \mathcal{M}_{\chi^{\prime}}^{\left(\mathfrak{p}_{i}\right)}\left(c^{\prime \vee}(y)\right)_{\eta} .
\end{gathered}
$$

We want to prove that $\pi_{A}^{*}$ of the second isomorphism is the first isomorphism under canonical identifications, and the only nontrivial part is to observe that

$$
\pi_{A}^{*}\left(\lambda_{A^{\prime}}^{\prime} \circ c^{\prime \vee}(y)\right)=\pi_{A}^{\vee} \circ \lambda_{A^{\prime}}^{\prime} \circ c^{\prime \vee}(y) \stackrel{c^{\vee \vee}=\pi_{A^{\circ}} c^{\vee}}{=} \pi_{A}^{\vee} \circ \lambda_{A^{\prime}}^{\prime} \circ \pi_{A} \circ c^{\vee}(y)=\xi \lambda_{A} \circ c^{\vee}(y) .
$$


We now want to compare the diagram (40) with

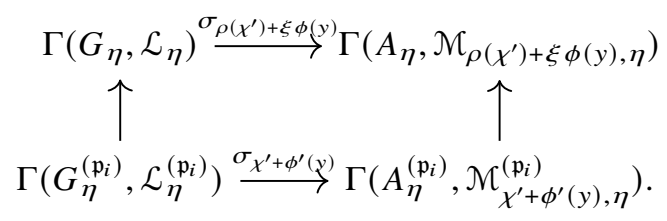

Recall that we have

$$
\sigma_{\rho\left(\chi^{\prime}\right)+\xi \phi(y)}=\psi(y) \tau\left(y, \rho\left(\chi^{\prime}\right)\right) T_{c^{\vee}(y)}^{*} \circ \sigma_{\rho\left(\chi^{\prime}\right)},
$$

where

$$
\psi(y): \mathcal{M}\left(c^{\vee}(y)\right)_{\eta} \stackrel{\sim}{\rightarrow} \mathcal{O}_{S, \eta}
$$

is a trivialisation of the fibre of $\mathcal{M}$ at $c^{\vee}(y)$ and

$$
\tau(y, \chi): \mathcal{O}_{\chi}\left(c^{\vee}(y)\right)_{\eta} \longrightarrow \mathcal{O}_{S, \eta}
$$

is a section of $\mathcal{O}_{\chi}\left(c^{\vee}(y)\right)_{\eta}^{\otimes-1}$ for each $y \in Y$ and $\chi \in X$, so that $\psi(y) \tau(y, \chi)$ is a section of $\mathcal{M}_{\chi}\left(c^{\vee}(y)\right)_{\eta}^{\otimes-1}$. Note that here we have tacitly changed the polarisation of $G$ from $\lambda$ to $\xi \lambda$, which has the effect of replacing $\phi$ by $\xi \phi$. This does not affect $\tau$ but may affect $\psi$, for which we use the same notation as before for simplicity.

Similarly, $\tau^{\prime}$ is characterised by the equation

$$
\sigma_{\chi^{\prime}+\phi^{\prime}(y)}=\psi^{\prime}(y) \tau^{\prime}\left(y, \chi^{\prime}\right) T_{c^{\prime v}(y)}^{*} \circ \sigma_{\chi^{\prime}}
$$

with isomorphism

$$
\psi^{\prime}(y): \mathcal{M}^{\left(\mathfrak{p}_{i}\right)}\left(c^{\prime \vee}(y)\right)_{\eta} \stackrel{\sim}{\rightarrow} \mathcal{O}_{S, \eta}
$$

and

$$
\tau^{\prime}\left(y, \chi^{\prime}\right): \mathcal{O}_{\chi^{\prime}}\left(c^{\prime \vee}(y)\right)_{\eta} \stackrel{\sim}{\rightarrow} \mathcal{O}_{S, \eta}
$$

so that $\psi^{\prime}(y) \tau^{\prime}\left(y, \chi^{\prime}\right)$ defines a section of $\mathcal{M}_{\chi^{\prime}}^{\left(\mathfrak{p}_{i}\right)}\left(c^{\prime \vee}(y)\right)_{\eta}^{\otimes-1}$.

Now (40), (41), (42) and (43) together imply that we have a commutative diagram

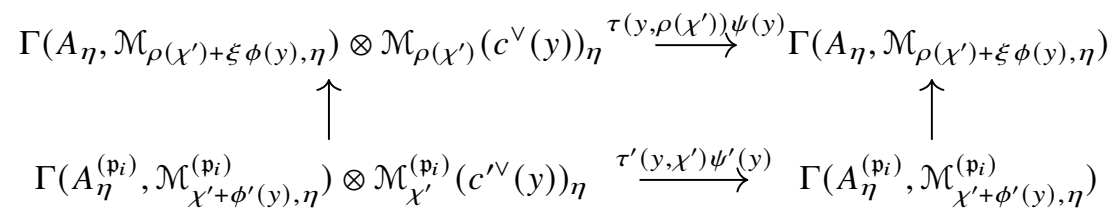

where we use that $\sigma_{\chi} \neq 0$ and $\sigma_{\chi^{\prime}} \neq 0$ for every $\chi \in X$ and $\chi^{\prime} \in X^{\prime}$. Observe that the vertical arrows are nonzero, and we obtain

$$
\tau^{\prime}\left(y, \chi^{\prime}\right)=\tau\left(y, \rho\left(\chi^{\prime}\right)\right)
$$

under the canonical identification $\mathcal{O}_{\rho\left(\chi^{\prime}\right)}\left(c^{\vee}(y)\right)_{\eta} \cong \pi_{A}^{*} \mathcal{O}_{\chi^{\prime}}\left(c^{\vee}(y)\right)_{\eta} \cong \mathcal{O}_{\chi^{\prime}}\left(\pi_{A} \circ c^{\vee}(y)\right)_{\eta}=\mathcal{O}_{\chi^{\prime}}\left(c^{\prime \vee}(y)\right)$, which completes the proof if we take the equivalent formulation of $\tau$ in 5.1.3. 


\subsection{Partial Frobenius extends to minimal compactifications}

In this final section, we deduce our main Theorem 3.16 from the theorem proved in the last section. We retain the setting of the last section, so in particular every scheme is defined over $\mathcal{O}_{F_{0}} \otimes_{\mathbb{Z}} \mathbb{F} p$.

We begin by recalling the construction of the minimal compactifications. In the analytic setting, the minimal compactifications can be constructed directly using rational boundary components. However, in the algebraic settings, the only known method to proceed is to first construct the toroidal compactifications and then contract the boundary to obtain the minimal compactifications.

More precisely, let $\omega^{\text {tor }}:=\bigwedge^{\text {top }} \underline{\mathrm{Lie}}_{G}^{\vee}{ }^{\text {tor }} / M_{n, \Sigma}^{\text {tor }}$, where $G^{\text {tor }}$ is the universal semi-abelian scheme over the toroidal compactification $M_{n, \Sigma}^{\text {tor }}$. Then $\omega^{\text {tor }}$ is an invertible sheaf generated by its global sections, and we define

$$
M_{n}^{\min }:=\operatorname{Proj}\left(\underset{k \geq 0}{\oplus} \Gamma\left(M_{n, \Sigma}^{\text {tor }},\left(\omega^{\text {tor }}\right)^{\otimes k}\right)\right)
$$

Alternatively, $M_{n}^{\min }$ is the Stein factorisation of the map

$$
M_{n, \Sigma}^{\text {tor }} \longrightarrow \mathbb{P}\left(\Gamma\left(M_{n, \Sigma}^{\text {tor }}, \omega^{\text {tor }}\right)\right)
$$

defined by global sections of $\omega^{\text {tor }}$; that is, it factors through

$$
\oint: M_{n, \Sigma}^{\mathrm{tor}} \rightarrow M_{n}^{\mathrm{min}}
$$

with $M_{n}^{\min } \rightarrow \mathbb{P}\left(\Gamma\left(M_{n, \Sigma}^{\text {tor }}, \omega^{\text {tor }}\right)\right)$ finite and

$$
\mathcal{O}_{M_{n}^{\min }} \stackrel{\sim}{\rightarrow} \oint_{*} \mathcal{O}_{M_{n, \Sigma}^{\mathrm{tor}}}
$$

It can be shown that $M_{n}^{\text {min }}$ is independent of the choice of the toroidal compactification. Moreover, by construction we have a canonical ample invertible sheaf $\omega^{\min }:=\mathcal{O}(1)$ on $M_{n}^{\min }$ such that

$$
\oint^{*} \omega^{\min } \cong \omega^{\text {tor }}
$$

We can show that $M_{n}^{\min }$ has a stratification

$$
M_{n}^{\min }=\bigsqcup_{\left[\left(Z_{n}, \Phi_{n}, \delta_{n}\right)\right]} Z_{\left[\left(Z_{n}, \Phi_{n}, \delta_{n}\right)\right]}
$$

where $Z_{\left[\left(Z_{n}, \Phi_{n}, \delta_{n}\right)\right]}=M_{n}^{Z_{n}}$ as defined in Subsection 5.3, and the index ranges through all cusp labels. Moreover, the map $\oint$ preserves the stratification and sends $Z_{\left[\left(\Phi_{n}, \delta_{n}, \sigma\right)\right]}$ to $Z_{\left[\left(Z_{n}, \Phi_{n}, \delta_{n}\right)\right]}$.

Similar to the toroidal compactifications, the minimal compactification of $M_{K(n)} / \Delta$ is defined to be the union of minimal compactifications of $M_{n}\left(L, \operatorname{Tr}_{\mathcal{O}_{F} / \mathbb{Z}} \circ\left(\alpha \delta\langle\cdot, \cdot\rangle_{F}\right)\right)$.

Theorem 5.28. $F_{\mathfrak{p}_{i}}$ extends to a morphism

$$
F_{\mathfrak{p}_{i}}^{\text {min }}:\left(M_{K(n)} / \Delta\right)^{\min } \longrightarrow\left(M_{K(n)} / \Delta\right)^{\min }
$$

sending the strata $M_{n}\left(L^{Z_{\alpha \delta, n}},\langle\cdot, \cdot\rangle^{Z_{\alpha \delta, n}}\right)$ associated to $\alpha \in \Omega, \delta \in \Lambda$ and the cusp label

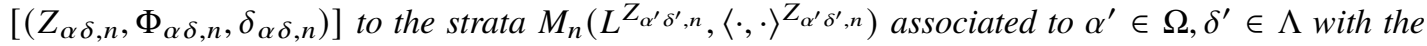
usual notations as before and the cusp label $\left[\left(Z_{\alpha^{\prime} \delta^{\prime}, n}, \Phi_{\alpha^{\prime} \delta^{\prime}, n}, \delta_{\alpha^{\prime} \delta^{\prime}, n}\right)\right]$ defined as follows:

$$
Z_{\alpha^{\prime} \delta^{\prime}, n}=Z_{\alpha \delta, n}
$$


If $\Phi_{\alpha \delta, n}=\left(X, Y, \phi, \varphi_{-2, n}, \varphi_{0, n}\right)$, then

$$
\Phi_{\alpha^{\prime} \delta^{\prime}, n}=\left(X \otimes_{\mathcal{O}_{F}} \mathfrak{p}_{i}, Y, \phi^{\prime}, \varphi_{-2, n}^{\prime}, \varphi_{0, n}^{\prime}\right)
$$

where

$$
\begin{gathered}
\varphi_{-2, n}^{\prime}: G r_{-2}^{Z_{\alpha^{\prime} \delta^{\prime}, n}}=G r_{-2}^{Z_{\alpha \delta, n}} \stackrel{\varphi_{-2, n}}{\longrightarrow} \operatorname{Hom}(X / n X,(\mathbb{Z} / n \mathbb{Z})(1)) \\
\stackrel{\sim}{\longrightarrow} \operatorname{Hom}\left(X \otimes \mathfrak{p}_{i} / n\left(X \otimes \mathfrak{p}_{i}\right),(\mathbb{Z} / n \mathbb{Z})(1)\right)
\end{gathered}
$$

and

$$
\varphi_{0, n}^{\prime}: G r_{0}^{Z_{\alpha^{\prime} \delta^{\prime}, n}}=G r_{0}^{Z_{\alpha \delta, n}} \stackrel{\varphi_{0, n}}{\longrightarrow} Y / n Y
$$

Lastly, $\phi^{\prime}$ is defined by the following diagram similar to the diagram defining $\lambda^{\prime}$ :

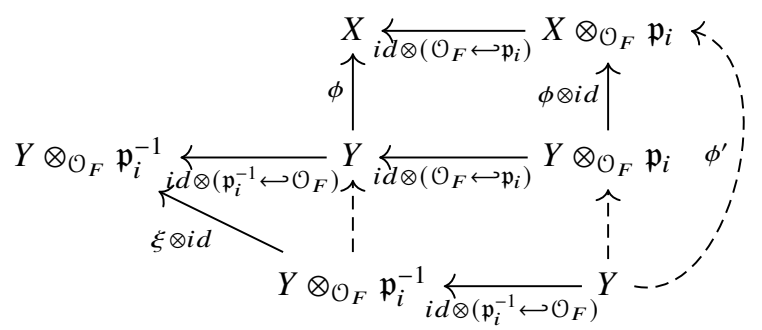

Moreover, on each strata, $F_{\mathfrak{p}_{i}}^{\text {min }}$ induces the morphism

$$
M_{n}\left(L^{Z_{\alpha \delta, n}},\langle\cdot, \cdot\rangle^{Z_{\alpha \delta, n}}\right) \rightarrow M_{n}\left(L^{Z_{\alpha^{\prime} \delta^{\prime}, n}},\langle\cdot, \cdot\rangle^{Z_{\alpha^{\prime} \delta^{\prime}, n}}\right)
$$

sending $\left(A, \lambda, i,\left(\alpha_{n}, v_{n}\right)\right)$ to $\left(A^{\prime}, \lambda^{\prime}, i^{\prime},\left(\alpha_{n}^{\prime}, v_{n}^{\prime}\right)\right)$ as in the description before the theorem. For completeness, we summarise the description as follows. Using the above notations, $A^{\prime}:=A /\left(\operatorname{Ker}(F)\left[\mathfrak{p}_{i}\right]\right), i^{\prime}$ is induced by the quotient map $\pi_{\mathfrak{p}_{i}}: A \rightarrow A^{\prime}, \lambda^{\prime}$ is characterised by $\xi \lambda=\pi_{\mathfrak{p}_{i}}^{\vee} \circ \lambda^{\prime} \circ \pi_{\mathfrak{p}_{i}}$, which defines a prime to $p$ isogeny $\lambda^{\prime}, \alpha_{n}^{\prime}=\pi_{\mathfrak{p}_{i}} \circ \alpha_{n}$ and $v_{n}^{\prime}=v_{n} \circ \kappa$. In other words, restriction of the partial Frobenius to (suitable union of) strata recovers the partial Frobenius on them.

Proof. It is enough to prove that $F_{\mathfrak{p}_{i}}$ extends to the minimal compactification of each component; that is,

$$
M_{n}\left(L, \operatorname{Tr}_{\mathcal{O}_{F} / \mathbb{Z}} \circ\left(\alpha \delta\langle\cdot, \cdot\rangle_{F}\right)\right) \rightarrow M_{n}\left(L, \operatorname{Tr}_{\mathcal{O}_{F} / \mathbb{Z}} \circ\left(\alpha^{\prime} \delta^{\prime}\langle\cdot, \cdot\rangle_{F}\right)\right)
$$

extends to a morphism

$$
M_{n}\left(L, \operatorname{Tr}_{\mathcal{O}_{F} / \mathbb{Z}} \circ\left(\alpha \delta\langle\cdot, \cdot\rangle_{F}\right)\right)^{\min } \rightarrow M_{n}\left(L, \operatorname{Tr}_{\mathcal{O}_{F} / \mathbb{Z}} \circ\left(\alpha^{\prime} \delta^{\prime}\langle\cdot, \cdot\rangle_{F}\right)\right)^{\min }
$$

and maps strata to the expected ones. We are thus reduced to the situation that we are familiar with.

We have morphisms

$$
\begin{gathered}
M_{n}\left(L, \operatorname{Tr}_{\mathcal{O}_{F} / \mathbb{Z}} \circ\left(\alpha \delta\langle\cdot, \cdot\rangle_{F}\right)\right)_{\Sigma_{\alpha \delta}}^{\text {tor }} \stackrel{F_{p_{i}}^{\text {tor }}}{\longrightarrow} M_{n}\left(L, \operatorname{Tr}_{\mathcal{O}_{F} / \mathbb{Z}} \circ\left(\alpha^{\prime} \delta^{\prime}\langle\cdot, \cdot\rangle_{F}\right)\right)_{\Sigma_{\alpha^{\prime} \delta^{\prime}}^{\prime}}^{\text {tor }} \\
\downarrow^{\downarrow} \quad \downarrow^{\prime} \\
M_{n}\left(L, \operatorname{Tr}_{\mathcal{O}_{F} / \mathbb{Z}} \circ\left(\alpha \delta\langle\cdot, \cdot\rangle_{F}\right)\right)^{\min } \stackrel{F_{p_{i}}^{\min }}{--}-M_{n}\left(L, \operatorname{Tr}_{\mathcal{O}_{F} / \mathbb{Z}} \circ\left(\alpha^{\prime} \delta^{\prime}\langle\cdot, \cdot\rangle_{F}\right)\right)^{\min }
\end{gathered}
$$

where the horizontal arrow is the extension of the partial Frobenius to toroidal compactifications as we have proved in the previous section and the dashed arrow is the morphism we are searching for that makes the diagram commute. Once the existence of the dashed arrow is established, the description of 
$F_{\mathfrak{p}_{i}}^{\min }$ follows from the commutativity of the diagram and the description of the first horizontal arrow as stated in the last section.

Let $G_{\Sigma_{\alpha \delta}}$ (respectively $\left.G_{\Sigma_{\alpha^{\prime} \delta^{\prime}}^{\prime}}\right)$ be the universal semi-abelian scheme over $M_{n, \Sigma_{\alpha \delta}}^{\text {tor }}:=M_{n}\left(L, \operatorname{Tr}_{\mathcal{O}_{F} / \mathbb{Z}^{\circ}}\right.$ $\left.\left(\alpha \delta\langle\cdot, \cdot\rangle_{F}\right)\right)_{\Sigma_{\alpha \delta}}^{\text {tor }}\left(\right.$ respectively $\left.M_{n, \Sigma_{\alpha^{\prime} \delta^{\prime}}^{\prime}}^{\text {tor }}:=M_{n}\left(L, \operatorname{Tr}_{\mathcal{O}_{F} / \mathbb{Z}} \circ\left(\alpha^{\prime} \delta^{\prime}\langle\cdot, \cdot\rangle_{F}\right)\right)_{\Sigma_{\alpha^{\prime} \delta^{\prime}}^{\prime}}^{\text {tor }}\right)$. Recall that $F_{p_{i}}^{\text {tor }}$ is characterised by

$$
\left(F_{\mathfrak{p}_{i}}^{\mathrm{tor}}\right)^{*} G_{\Sigma_{\alpha^{\prime} \delta^{\prime}}^{\prime}} \cong G_{\Sigma_{\alpha \delta}}^{\left(\mathfrak{p}_{i}\right)}:=G_{\Sigma_{\alpha \delta}} /\left(\operatorname{Ker}(F)\left[\mathfrak{p}_{i}\right]\right)
$$

hence,

$$
\left(F_{\mathfrak{p}_{i}}^{\mathrm{tor}}\right)^{*} \underline{\mathrm{Lie}}_{G_{\Sigma_{\alpha^{\prime} \delta^{\prime}}}^{\vee}}^{\vee} / M_{n, \Sigma_{\alpha^{\prime} \delta^{\prime}}}^{\mathrm{tor}} \cong \underline{\mathrm{Lie}}_{G_{\Sigma_{\alpha \delta}}^{\vee}}^{\left(\mathrm{p}_{i}\right)} / M_{n, \Sigma_{\alpha \delta}}^{\mathrm{tor}}
$$

Because the action of $\mathcal{O}_{F}$ on $\underline{\mathrm{Lie}}_{G_{\Sigma_{\alpha \delta}} / M_{n, \Sigma_{\alpha \delta}}^{\mathrm{tor}}}^{\vee}$ (respectively $\underline{\mathrm{Lie}}_{G_{\Sigma_{\alpha^{\prime}}^{\prime} \delta^{\prime}}^{\vee}}^{\vee} M_{n, \Sigma_{\alpha^{\prime} \delta^{\prime}}}^{\mathrm{tor}}$ ) factors through $\mathcal{O}_{F} / p \cong$ $\prod_{i} \mathcal{O}_{F} / \mathfrak{p}_{i}$, we have

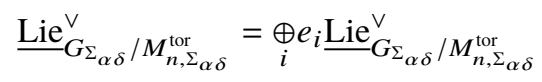

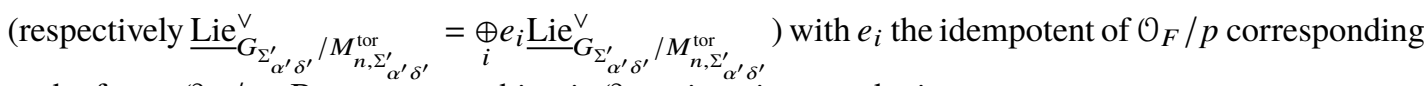
to the factor $\mathcal{O}_{F} / \mathfrak{p}_{i}$. Because everything is $\mathcal{O}$-equivaraint, we obtain

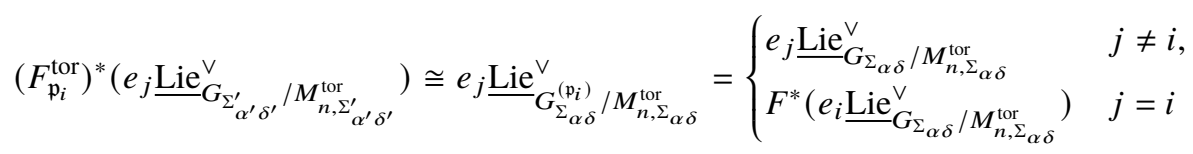

where the last equality follows from

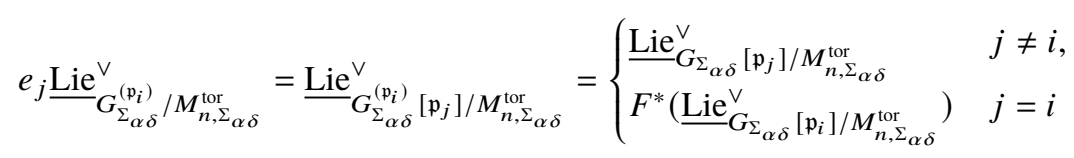

in which we use

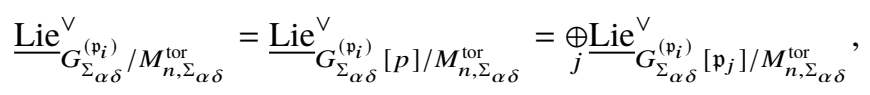

and similarly for $\underline{\mathrm{Lie}}_{G_{\Sigma_{\alpha \delta}}}^{\vee} / M_{n, \Sigma_{\alpha \delta}}^{\text {tor }}$, together with

$$
G_{\Sigma_{\alpha \delta}}^{\left(\mathfrak{p}_{i}\right)}\left[\mathfrak{p}_{j}\right]= \begin{cases}G_{\Sigma_{\alpha \delta}}\left[\mathfrak{p}_{j}\right] & j \neq i \\ G_{\Sigma_{\alpha \delta}}^{(p)}\left[\mathfrak{p}_{i}\right] & j=i\end{cases}
$$

where $G_{\Sigma_{\alpha \delta}}^{(p)}:=\left(G_{\Sigma_{\alpha \delta}} / \operatorname{Ker}(F)\right)$ is the usual base change by the absolute Frobenius $F$ on $M_{n, \Sigma_{\alpha \delta}}^{\text {tor }}$.

Let $\omega_{i}:=\bigwedge^{\text {top }} e_{i} \underline{\mathrm{Lie}}_{G_{\Sigma_{\alpha \delta}}}^{\vee} / M_{n, \Sigma_{\alpha \delta}}^{\text {tor }}$ (respectively $\omega_{i}^{\prime}:=\bigwedge^{\text {top }} e_{i} \underline{\operatorname{Lie}}_{G_{\Sigma_{\alpha^{\prime} \delta^{\prime}}^{\prime}}^{\vee} / M_{n, \Sigma_{\alpha^{\prime} \delta^{\prime}}}^{\text {tor }}}$ ), then we have

$$
\omega=\underset{i}{\otimes} \omega_{i}
$$

and

$$
\omega^{\prime}=\underset{i}{\otimes} \omega_{i}^{\prime}
$$


with $\omega:=\bigwedge^{\text {top }} \underline{\mathrm{Lie}}_{G_{\Sigma_{\alpha \delta}}}^{\vee} / M_{n, \Sigma_{\alpha \delta}}^{\text {tor }}$ and $\omega^{\prime}:=\bigwedge^{\text {top }} \underline{\operatorname{Lie}}_{G_{\Sigma_{\alpha^{\prime} \delta^{\prime}}}^{\vee} / M_{n, \Sigma_{\alpha^{\prime} \delta^{\prime}}}^{\mathrm{tor}}}^{\mathrm{ar}}$ as before. Moreover, (45) tells us that

$$
F_{\mathfrak{p}_{i}}^{\mathrm{tor} *} \omega_{j}^{\prime}= \begin{cases}\omega_{j} & j \neq i \\ F^{*} \omega_{i}=\omega_{i}^{p} & j=i\end{cases}
$$

hence,

$$
F_{\mathfrak{p}_{i}}^{\mathrm{tor} *} \omega^{\prime}=\left(\underset{j \neq i}{\otimes} \omega_{j}\right) \otimes \omega_{i}^{p}
$$

If we can show that each $\omega_{i}$ descends to a line bundle on

$$
M_{n, \alpha \delta}^{\min }:=M_{n}\left(L, \operatorname{Tr}_{\mathcal{O}_{F} / \mathbb{Z}} \circ\left(\alpha \delta\langle\cdot, \cdot\rangle_{F}\right)\right)^{\min }
$$

through $\oint-$ that is, there exists a line bundle $\omega_{i}^{\min }$ on $M_{n, \alpha \delta}^{\min }$ such that

$$
\oint^{*} \omega_{i}^{\min }=\omega_{i}
$$

then $\left(F_{\mathfrak{p}_{i}}^{\mathrm{tor}}\right)^{*} \omega^{\prime}=\left(\underset{j \neq i}{\otimes} \omega_{j}\right) \otimes \omega_{i}^{p}$ tells us that we can find a line bundle $L^{\mathrm{min}}:=\left(\underset{j \neq i}{\otimes} \omega_{j}^{\min }\right) \otimes\left(\omega_{i}^{\min }\right)^{p}$ such that

$$
\oint^{*} L^{\min }=\left(F_{\mathfrak{p}_{i}}^{\text {tor }}\right)^{*} \omega^{\prime}
$$

and the universal property of Proj construction tells us that there exists $F_{\mathfrak{p}_{i}}^{\min }$, which makes the diagram (44) commutative.

Indeed, recall that the universal property of the Proj construction is as follows. Let $\mathcal{A}=\underset{k \geq 0}{\oplus} \mathcal{A}_{k}$ be a graded $R$-algebra finitely generated by degree 1 elements and $T$ be a scheme defined over $R$ with structure map $f: T \rightarrow \operatorname{Spec}(R)$. Suppose we are given a line bundle $\mathcal{L}$ on $T$ and a morphism of graded $R$-algebras

$$
\psi: \mathcal{A} \rightarrow f_{*}\left(\underset{k \geq 0}{\oplus} \mathcal{L}^{\otimes k}\right)=\underset{k \geq 0}{\oplus} \Gamma\left(T, \mathcal{L}^{\otimes k}\right)
$$

whose adjoint morphism at degree $1 f^{*} \mathcal{A}_{1} \rightarrow \mathcal{L}$ is surjective (viewing $\mathcal{A}_{1}$ as a quasi-coherent module on $\operatorname{Spec}(R)$ ), then there exists a unique morphism

$$
g: T \longrightarrow \operatorname{Proj}_{R}(\mathcal{A})
$$

of $R$-schemes together with an isomorphism

$$
\theta: g^{*} \mathcal{O}(1) \cong \mathcal{L}
$$

such that $\psi$ factorises as

$$
\psi: \mathcal{A} \cong \underset{k \geq 0}{\oplus} \Gamma\left(\operatorname{Proj}_{R}(\mathcal{A}), \mathcal{O}(1)^{\otimes k}\right) \stackrel{g^{*}}{\rightarrow} \underset{k \geq 0}{\oplus} \Gamma\left(T, g^{*} \mathcal{O}(1)^{\otimes n}\right) \stackrel{\theta}{\cong} \underset{k \geq 0}{\oplus} \Gamma\left(T, \mathcal{L}^{\otimes n}\right) .
$$

In our setting, $\oint^{\prime} \circ F_{\mathfrak{p}_{i}}^{\text {tor }}$ is induced by

$$
\underset{k \geq 0}{\oplus} \Gamma\left(M_{n, \Sigma_{\alpha^{\prime} \delta^{\prime}}^{\prime}}^{\text {tor }}, \omega^{\prime \otimes k}\right) \stackrel{\left(F_{\mathfrak{p}_{i}}^{\text {tor }}\right)^{*}}{\rightarrow} \underset{k \geq 0}{\oplus} \Gamma\left(M_{n, \Sigma_{\alpha \delta}}^{\text {tor }},\left(\left(F_{\mathfrak{p}_{i}}^{\text {tor }}\right)^{*} \omega^{\prime}\right)^{\otimes k}\right)
$$


with $\mathcal{L}=\left(F_{p_{i}}^{\text {tor }}\right)^{*} \omega^{\prime}$. Assume that we know the existence of $\omega_{i}^{\text {min }}$, then we have $L^{\text {min }}$ such that $\oint^{*} L^{\text {min }}=$ $\left(F_{\mathfrak{p}_{i}}^{\text {tor }}\right)^{*} \omega^{\prime}$. Because $\mathcal{O}_{M_{n, \alpha \delta}^{\min }} \stackrel{\sim}{\rightarrow} \oint_{*} \mathcal{O}_{M_{n, \Sigma}^{\text {tor }} \text { to }}$, we have by projection formula

$$
\oint_{*} \oint^{*} L^{\mathrm{min}}=L^{\mathrm{min}} \otimes \oint_{*} \mathcal{O}_{M_{n, \Sigma_{\alpha \delta}}^{\mathrm{tor}}}=L^{\mathrm{min}} \otimes \mathcal{O}_{M_{n, \alpha \delta}^{\min }}=L^{\mathrm{min}}
$$

(note that because $L^{\mathrm{min}}$ is locally free, the derived tensor prodoct and left derived pullback is just the usual one), which implies that

$$
\Gamma\left(M_{n, \alpha \delta}^{\min }, L^{\min }\right) \stackrel{\oint^{*}}{\cong} \Gamma\left(M_{n, \Sigma_{\alpha \delta}}^{\text {tor }}, \oint^{*} L^{\min }\right) \cong \Gamma\left(M_{n, \Sigma_{\alpha \delta}}^{\text {tor }},\left(F_{\mathfrak{p}_{i}}^{\text {tor }}\right)^{*} \omega^{\prime}\right)
$$

and similar for $\left(L^{\mathrm{min}}\right)^{\otimes k}$. Thus, (46) gives us a morphism

$$
\underset{k \geq 0}{\oplus} \Gamma\left(M_{n, \Sigma_{\alpha^{\prime} \delta^{\prime}}^{\text {tor }}}^{\prime}, \omega^{\otimes k}\right) \rightarrow \underset{k \geq 0}{\oplus} \Gamma\left(M_{n, \alpha \delta}^{\min },\left(L^{\min }\right)^{\otimes k}\right),
$$

which by the universal property of Proj construction induces a morphism

$$
F_{\mathfrak{p}_{i}}^{\min }: M_{n, \alpha \delta}^{\min } \longrightarrow M_{n, \alpha^{\prime} \delta^{\prime}}^{\min }:=\operatorname{Proj}\left(\underset{k \geq 0}{\oplus} \Gamma\left(M_{n, \Sigma_{\alpha^{\prime} \delta^{\prime}}^{\prime}}^{\mathrm{tor}}, \omega^{\prime \otimes k}\right)\right),
$$

which makes the diagram (44) commutative.

Thus, we are reduced to showing the existence of $\omega_{i}^{\min }$ such that $\oint^{*} \omega_{i}^{\min }=\omega_{i}$. Let $M_{n, \alpha \delta}^{1} \subset M_{n, \alpha \delta}^{\min }$ be the union of the open stratum and all of the codimension 1 strata, then it follows from [18] Proposition 7.2.3.13 that

$$
\oint: \oint^{-1}\left(M_{n, \alpha \delta}^{1}\right) \cong M_{n, \alpha \delta}^{1}
$$

so we can view $M_{n, \alpha \delta}^{1}$ as an open subscheme of $M_{n, \Sigma_{\alpha \delta}}^{\text {tor }}$ as well. Let

$$
\omega_{i}^{\min }:=\left(M_{n, \alpha \delta}^{1} \hookrightarrow M_{n, \alpha \delta}^{\min }\right)_{*}\left(\left.\omega_{i}\right|_{M_{n, \alpha \delta}^{1}}\right),
$$

then we will show that $\omega_{i}^{\min }$ is a line bundle and $\oint^{*} \omega_{i}^{\min } \cong \omega_{i}$. This is a direct adaption of the proof of [18] Theorem 7.2.4.1 in our case.

First observe that $\omega_{i}^{\min }$ is a coherent sheaf because $M_{n, \alpha \delta}^{\min }$ is normal and the complement of $M_{n, \alpha \delta}^{1}$ has codimension at least 2 ([13] VIII Proposition 3.2). Then to show that it is a line bundle, it is enough to show that its stalk at every point is free of rank 1. By fpqc descent, it is enough to show this for the completions of the strict localisations of $M_{n, \alpha \delta}^{\min }$; that is, it is enough to prove that for every geometric point $\bar{x}$ of $M_{n, \alpha \delta}^{\min }$, the pullback of $\omega_{i}^{\min }$ to $\left(M_{n, \alpha \delta}^{\min }\right)_{\bar{x}}^{\wedge}$, the completions of the strict localisation of $M_{n, \alpha \delta}^{\min }$ at $\bar{x}$, is free of rank 1. Similarly, it is enough to prove that $\oint^{*} \omega_{i}^{\min } \cong \omega_{i}$ holds naturally over $\left(M_{n, \Sigma_{\alpha \delta}}^{\text {tor }}\right)_{\bar{y}}^{\wedge}$ for every geometric point $\bar{y}$ of $M_{n, \Sigma_{\alpha \delta}}^{\text {tor }}$.

Suppose that $\bar{x}$ lies in the stratum $Z_{\left[\left(Z_{n}, \Phi_{n}, \delta_{n}\right)\right]}$, and we choose a stratum $Z_{\left[\left(\Phi_{n}, \delta_{n}, \sigma\right)\right]}$ lying above $Z_{\left[\left(Z_{n}, \Phi_{n}, \delta_{n}\right)\right]}$. Then from (2) of Theorem 5.19 we have a natural identification

$$
\left(M_{n, \Sigma_{\alpha \delta}}^{\text {tor }}\right)_{Z_{\left[\left(\Phi_{n}, \delta_{n}, \sigma\right)\right]}}^{\wedge} \cong \mathfrak{X}_{\Phi_{n}, \delta_{n}, \sigma}
$$

where we do not have the quotient by $\Gamma_{\Phi_{n}}, \sigma$ because we assume that $n>3$. We have a canonical map $\mathfrak{X}_{\Phi_{n}, \delta_{n}, \sigma} \rightarrow\left(M_{n, \alpha \delta}^{\min }\right)_{Z_{\left[\left(Z_{n}, \Phi_{n}, \delta_{n}\right)\right]}}^{\wedge}$ induced by $\oint$, and by abuse of notation we let

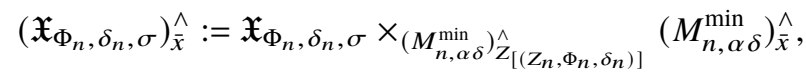


so by definition we have a morphism

$$
\left(\mathfrak{X}_{\Phi_{n}, \delta_{n}, \sigma}\right)_{\bar{x}}^{\wedge} \rightarrow\left(M_{n, \alpha \delta}^{\min }\right)_{\bar{x}}^{\wedge} .
$$

The key point is that we have a morphism

$$
\left(M_{n, \alpha \delta}^{\min }\right)_{\bar{x}}^{\wedge} \rightarrow\left(M_{n}^{Z_{n}}\right)_{\bar{x}}^{\wedge}
$$

such that the composition

$$
\left(\mathfrak{X}_{\Phi_{n}, \delta_{n}, \sigma}\right)_{\bar{x}}^{\wedge} \rightarrow\left(M_{n, \alpha \delta}^{\min }\right)_{\bar{x}}^{\wedge} \rightarrow\left(M_{n}^{Z_{n}}\right)_{\bar{x}}^{\wedge}
$$

is induced by the structural morphism $p: \mathfrak{X}_{\Phi_{n}, \delta_{n}, \sigma} \rightarrow M_{n}^{Z_{n}}$ (recall that $\mathfrak{X}_{\Phi_{n}, \delta_{n}, \sigma}$ is the formal completion along the boundary of an affine toroidal compactification of a torus torsor over an abelian scheme over $M_{n}^{Z_{n}}$ ); see [18] Proposition 7.2.3.16 for details.

We observe that the pullback of the line bundle $\omega_{i}$ over $\mathfrak{X}_{\Phi_{n}, \delta_{n}, \sigma}$ is canonically identified with $\left(\bigwedge_{\mathbb{Z}}^{\text {top }} e_{i} X\right) \otimes_{\mathbb{Z}} p^{*}\left(\bigwedge^{\text {top }} e_{i} \underline{\operatorname{Lie}}_{A / M_{n}}^{Z_{n}}\right)$, where $A$ is the universal abelian variety over $M_{n}^{Z_{n}}$. This is a trivial variant of [18] Lemma 7.1.2.1, and we briefly recall the proof. By étale descent, we can assume that the base is $S=\operatorname{Spf}(R, I)$, with $R$ normal Noetherian and $I$-adically complete so that we are in the setting of Subsection 5.2.2. We have

$$
\underline{\mathrm{Lie}}_{G}^{\vee}{ }_{\text {for }}^{\vee}=\underline{\mathrm{Lie}}_{G_{\text {for }}^{\natural} / S}^{\vee}
$$

hence,

$$
\omega_{i}=\bigwedge^{\text {top }} e_{i} \underline{\mathrm{Lie}}_{G_{\text {for }} / S}^{\vee}=\bigwedge^{\text {top }} e_{i} \underline{\mathrm{Lie}}_{G_{\text {for }}^{\natural} / S}^{\vee}=\left(\bigwedge_{\mathbb{Z}}^{\text {top }} e_{i} X\right) \otimes_{\mathbb{Z}} \bigwedge^{\text {top }} e_{i} \underline{\mathrm{Lie}}_{A_{\text {for }} / S},
$$

where the last equality follows from the short exact sequence

$$
0 \rightarrow e_{i} \underline{\mathrm{Lie}}_{T / S} \rightarrow e_{i} \underline{\mathrm{Lie}}_{G \text { घ/S }} \rightarrow e_{i} \underline{\mathrm{Lie}}_{A / S} \rightarrow 0
$$

induced by the global semi-abelian structure

$$
0 \rightarrow T \rightarrow G^{\natural} \rightarrow A \rightarrow 0
$$

of $G^{\natural}$.

From what we have seen, the restriction of $\omega_{i}$ to $\left(\mathfrak{X}_{\Phi_{n}, \delta_{n}, \sigma}\right)_{\bar{x}}^{\wedge}$ is the pullback of $\left(\bigwedge_{\mathbb{Z}}^{\text {top }} e_{i} X\right) \otimes_{\mathbb{Z}}$ $\left(\bigwedge^{\text {top }} e_{i} \underline{\operatorname{Lie}}_{A / M_{n}^{\vee}}^{Z_{n}}\right.$ ) along the composition

$$
\left(\mathfrak{X}_{\Phi_{n}, \delta_{n}, \sigma}\right)_{\bar{x}}^{\wedge} \rightarrow\left(M_{n, \alpha \delta}^{\min }\right)_{\bar{x}}^{\wedge} \rightarrow\left(M_{n}^{Z_{n}}\right)_{\bar{x}}^{\wedge}
$$

which in particular shows that it is the pullback of some line bundle $L$ on $\left(M_{n, \alpha \delta}^{\min }\right)_{\bar{x}}^{\wedge}$; that is, by abuse of notation,

$$
\left(\oint_{\bar{x}}^{\wedge}\right)^{*} L \cong\left(\omega_{i}\right)_{\bar{x}}^{\wedge}
$$

This implies that both $\left(\omega_{i}^{\min }\right)_{\bar{x}}^{\wedge}$ and $L$ are extensions of (the completion of the strict localisation at $\bar{x}$ of $)\left.\omega_{i}\right|_{M_{n, \alpha \delta}^{1}}$, which by Stacks Project 30.12 .12 is equivalent $\left(\left(\omega_{i}^{\min }\right)_{\bar{x}}^{\wedge}\right.$ is reflexive because it is the pushforward of an open embedding and $L$ is reflexive because it is a line bundle on a normal scheme). 
This proves that $\left(\omega_{i}^{\min }\right)_{\bar{x}}^{\wedge}$ is free of rank 1 and for every geometric point $\bar{y}$ of $Z_{\left[\left(\Phi_{n}, \delta_{n}, \sigma\right)\right]}$ with $\bar{x}=\oint(\bar{y})$ we have a natural map $h:\left(M_{n, \Sigma_{\alpha \delta}}^{\text {tor }}\right)_{\bar{y}}^{\wedge} \rightarrow\left(\mathfrak{X}_{\Phi_{n}, \delta_{n}, \sigma}\right)_{\bar{x}}^{\wedge}$ and

$$
\left(\oint^{*}\left(\omega_{i}^{\min }\right)\right)_{\bar{y}}^{\wedge} \cong h^{*}\left(\oint_{\bar{x}}^{\wedge}\right)^{*}\left(\omega_{i}^{\min }\right)_{\bar{x}}^{\wedge} \stackrel{(47)}{\cong} h^{*}\left(\left(\omega_{i}\right)_{\bar{x}}^{\wedge}\right) \cong\left(\omega_{i}\right)_{\bar{y}}^{\wedge}
$$

proving what we want.

To be more precise, there are canonical morphisms

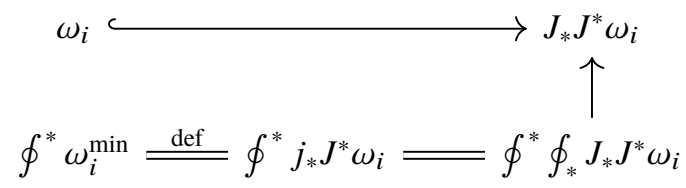

where $j$ and $J$ are open embeddings defined by the diagram

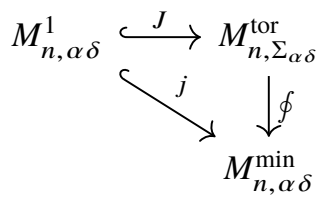

and the two arrows are the adjunction morphism. We showed that $\oint^{*} \omega_{i}^{\min }$ and $\omega_{i}$ are naturally identified over $\left(M_{n, \Sigma_{\alpha \delta}}^{\text {tor }}\right)_{\bar{y}}^{\wedge}$ for every geometric point $\bar{y}$ of $M_{n, \Sigma_{\alpha \delta}}^{\text {tor }}$, and the naturality tells us that after localisation and completion, the images of $\omega_{i}$ and $\oint^{*} \omega_{i}^{\min }$ in $J_{*} J^{*} \omega_{i}$ are identified. Now we can apply the fpqc descent to those two image sheaves and conclude that $\oint^{*} \omega_{i}^{\min } \cong \omega_{i}$.

Acknowledgements. This work is the author's PhD thesis at the University of Cambridge under the support of Cambridge Trust. The author thanks his PhD supervisor Tony Scholl for the guidance and encouragement he has received during the past four years. The author is grateful to Marius Leonhardt, who raised his interest in plectic theory. The author also thanks Jack Thorne and Ana Caraiani for a careful reading of this article and suggestions for improvement.

Conflict of Interest: None.

\section{References}

[1] Joseph Ayoub and Steven Zucker, 'Relative Artin motives and the reductive Borel-Serre compactification of a locally symmetric variety', Invent. Math., 188(2) (2012), 277-427.

[2] A. A. Beřlinson, J. Bernstein and P. Deligne, 'Faisceaux pervers', in Analysis and Topology on Singular Spaces, I (Luminy, 1981), Vol. 100 of Astérisque (Société Mathématique de France, Paris, 1982), 5-171.

[3] Don Blasius, 'Hilbert modular forms and the Ramanujan conjecture', in Noncommutative Geometry and Number Theory (Springer, Vieweg 2006), 35-56.

[4] Don Blasius and Jonathan D. Rogawski, 'Motives for Hilbert modular forms', Invent. Math., 114(1) (1993), 55-87.

[5] José I. Burgos and Jörg Wildeshaus, 'Hodge modules on Shimura varieties and their higher direct images in the Baily-Borel compactification', in Annales Scientifiques de l'Ecole Normale Supérieure, Vol. 37 (Elsevier, 2004), 363-413.

[6] P. Deligne, Cohomologie étale, Vol. 569 of Lecture Notes in Mathematics (Springer, Berlin, 1977).

[7] Pierre Deligne and Travaux de Shimura, Lecture Notes in Math., 244 (1971), 123-165.

[8] Pierre Deligne. Théorie de Hodge: II. Publications Mathématiques de l'IHÉS, Tome 40 (1971), pp. 5-57.

[9] Pierre Deligne. La conjecture de Weil: II. Publications Mathématiques de l'IHÉS, Tome 52 (1980), pp. 137-252.

[10] Mladen Dimitrov, 'Compactifications arithmétiques des variétés de Hilbert et formes modulaires de Hilbert pour $\Gamma_{1}(c, n)$, in Geometric Aspects of Dwork Theory, Vols. I, II (Walter de Gruyter, Berlin, 2004), 527-554.

[11] Gerd Faltings and Ching-Li Chai Degeneration of abelian varieties With an appendix by David Mumford of Ergebnisse der Mathematik und ihrer Grenzgebiete (3) [Results in Mathematics and Related Areas (3)] (Springer-Verlag, Berlin, 1990), xii+316.

[12] Eberhard Freitag, Hilbert Modular Forms (Springer, Berlin, 1990). 
[13] Alexander Grothendieck, Cohomologie locale des faisceaux cohérents et théorèmes de Lefschetz locaux et globaux (NorthHolland Publishing Co., Amsterdam; Masson \& Cie, Éditeur, Paris, 1968).

[14] Haruzo Hida, p-Adic Automorphic Forms on Shimura Varieties. Springer Monographs in Mathematics (Springer, New York, 2004).

[15] Luc Illusie, Yves Laszlo and Fabrice Orgogozo, eds., Travaux de Gabber sur l'uniformisation locale et la cohomologie étale des schémas quasi-excellents (Société Mathématique de France, Paris, 2014), 363-364. With the collaboration of Frédéric Déglise, Alban Moreau, Vincent Pilloni, Michel Raynaud, Joël Riou, Benoît Stroh, Michael Temkin and Weizhe Zheng.

[16] Naoki Imai and Yoichi Mieda, 'Toroidal compactifications of Shimura varieties of PEL type and its applications', in Algebraic Number Theory and Related Topics 2011 Research Institute for Mathematical Sciences, Kyoto, 2013), 3-24.

[17] Robert E. Kottwitz, 'Points on some Shimura varieties over finite fields' J. Amer. Math. Soc., 5(2) (1992), $373-444$.

[18] Kai-Wen Lan, Arithmetic Compactifications of PEL-Type Shimura Varieties, Number 36 (Princeton University Press, Princeton, NJ, 2013).

[19] Kai-Wen Lan and Benoît Stroh, 'Nearby cycles of automorphic étale sheaves', Compos. Math., 154(1) (2018), 80-119.

[20] Sophie Morel, 'Cohomologie d'intersection des variétés modulaires de Siegel suite', Compos. Math., 147(6) (2011), 16711740.

[21] Sophie Morel, 'Mixed $\ell$-adic complexes for schemes over number fields', (2019), Preprint.

[22] David Mumford, Abelian Varieties. Tata Institute of Fundamental Research Studies in Mathematics, No. 5 (Oxford University Press, London, 1970).

[24] Arvind N. Nair, 'Mixed structures in Shimura varieties and automorphic forms, Preprint.

[25] Jan Nekovár, 'Eichler-Shimura relations and semisimplicity of étale cohomology of quaternionic Shimura varieties', Ann. Sci. de l'École Norm. Supérieure. Quatrième Série, 51(5) (2018), 1179-1252.

[26] J. Nekováŕ and A. J. Scholl, 'Introduction to plectic cohomology', in Advances in the Theory of Automorphic Forms and Their L-Functions, Vol. 664 of Contemp. Math., (American Mathematical Society, Providence, RI, 2016) 321-337.

[27] J. Nekováŕ and A. J. Scholl, Plectic Hodge Theory I (2017), Preprint.

[28] Richard Pink, Arithmetical Compactification of Mixed Shimura Varieties, Vol. 209 of Bonner Mathematische Schriften [Bonn Mathematical Publications] (Universität Bonn, Mathematisches Institut, Bonn, Germany, 1990).

[29] Richard Pink, 'On $l$-adic sheaves on Shimura varieties and their higher direct images in the Baily-Borel compactification', Math. Ann., 292(2) (1992), 197-240.

[30] Yichao Tian and Liang Xiao, 'p-Adic cohomology and classicality of overconvergent Hilbert modular forms', Astérisque, 382 (2016), 73-162. The Stacks Project, Available at http://stacks.math.columbia.edu.

[31] Jörg Wildeshaus, 'On the interior motive of certain Shimura varieties: the case of Hilbert-Blumenthal varieties', Int. Math. Res. Not. IMRN (10), 2012 (2012), 2321-2355. 